\title{
Acoustic Scattering from Sand Dollars (Dendraster excentricus): Modeling as High Aspect Ratio Oblate Objects and Comparison to Experiment
}

\author{
by \\ Gregory C. Dietzen \\ B.S., United States Naval Academy, 2006 \\ Submitted in partial fulfillment of the requirements of the degree of \\ Master of Science \\ at the \\ MASSACHUSETTS INSTITUTE OF TECHNOLOGY \\ And the \\ WOODS HOLE OCEANOGRAPHIC INSTITUTION \\ September 2008 \\ (C) Gregory C. Dietzen, 2008. All rights reserved.
}

The author hereby grants to MIT and WHOI permission to reproduce and to distribute publicly paper and electronic copies of this thesis document in whole or in part in any medium now known or hereafter created.

Author

Joint Program in Oceanography/Applied Ocean Science and Engineering September 2008

Certified by

Timothy K. Stanton, Senior Scientist

Thesis Co-Supervisor

Certified by

Andone C. Lavery, Associate Scientist

Thesis Co-Supervisor

Accepted by

Alex Techet, Associate Professor of Mechanical Engineering

Chair, Joint Committee for Applied Ocean Science and Engineering

Accepted by

Lallit Anand, Professor of Mechanical Engineering Chairman, Graduate Committee 


\title{
Acoustic Scattering from Sand Dollars (Dendraster excentricus): Modeling as High Aspect Ratio Oblate Objects and Comparison to Experiment \\ by \\ Gregory C. Dietzen
}

\author{
Submitted in partial fulfillment of the requirements of the degree of \\ Master of Science \\ at the Massachusetts Institute of Technology \\ and the Woods Hole Oceanographic Institution
}

\begin{abstract}
Benthic shells can contribute greatly to the scattering variability of the ocean bottom, particularly at low grazing angles. Among the effects of shell aggregates are increased scattering strength and potential subcritical angle penetration of the seafloor. Sand dollars (Dendraster excentricus) occur commonly in the ocean and have been shown to be significant scatters of sound. In order to understand more fully the scattering mechanisms of these organisms, the scattering from individual sand dollars was studied using several methods.

Using an approximation to the Helmholtz-Kirchhoff integral, the Kirchhoff method gives an analytic integral expression to the backscattering from an object. This integral was first solved analytically for a disk and a spherical cap, two high aspect ratio oblate shapes which simplify the shape of an individual sand dollar. A method for solving the Kirchhoff integral numerically was then developed. An exact three dimensional model of a sand dollar test was created from computed tomography scans. The Kirchhoff integral was then solved numerically for this model of the sand dollar.

The finite element method, a numerical technique for approximating the solutions to partial differential equations and integral equations, was used to model the scattering from an individual sand dollar as well. COMSOL Multiphysics was used for the implementation of the finite element method.

Modeling results were compared with published laboratory experimental data from the free field scattering of both an aluminum disk and a sand dollar. Insight on the scattering mechanisms of individual sand dollar, including elastic behavior and diffraction effects, was gained from these comparisons.
\end{abstract}

Thesis Co-Supervisor: Tim Stanton

Title: Senior Scientist

Thesis Co-Supervisor: Andone Lavery

Title: Associate Scientist 


\section{Acknowledgements}

The chance to pursue scientific research at the Massachusetts Institute of Technology and the Woods Hole Oceanographic Institution for the past two years has fulfilled a dream. I will forever be grateful to the Navy for providing me the opportunity and the funding to make this possible. It has been time well spent!

I am extremely appreciative of the support of my two research advisors at the Woods Hole Oceanographic Institution. Dr. Tim Stanton provided the framework for my work at WHOI with his immense experience in the field of acoustic scattering. The enthusiasm and acumen that he offered have been truly valuable gifts. Dr. Andone Lavery has been a constant source of guidance and tutelage, helping me clear many difficult research hurdles. Her insight and tireless assistance were always more than enough to encourage me to continue forward.

This thesis would not have been possible without the help of Dr. Gonzalo Feijoo. His generous donations of time and energy helped introduce me to the powerful and challenging world of modern numerical analysis. Without his insight and contribution of computer resources, the necessary finite element method calculations would have been impossible. I am also grateful to Dr. Dezhang Chu who provided the experimental data that was such a key component of my work.

I would not be where I am today without my parents, who very early taught me the value of filling "the unforgiving minute with sixty seconds worth of distance run." Their unwavering love and support have been a steadfast source of strength. The rest of my family, especially my sister Mary Devon and brother Peter, have continued to help with challenges on the road ahead by reminding me of where I come from.

So many friends, old and new, have been a part of my life over the past two years. I know I will always remember fondly the time that I spent with them in Cambridge and Woods Hole. Finally I would like to thank my amazing girlfriend, Maya, for her encouragement and support in everything that I do. Daisuki desu. 


\section{Contents}

CHAPTER 1 INTRODUCTION........................................................................................................................11

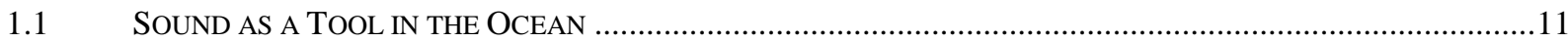

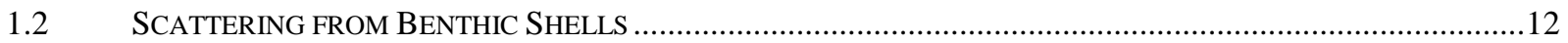

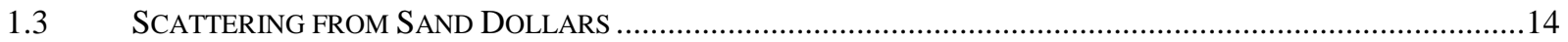

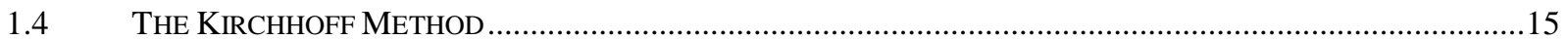

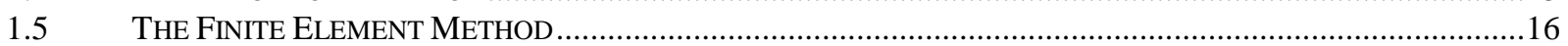

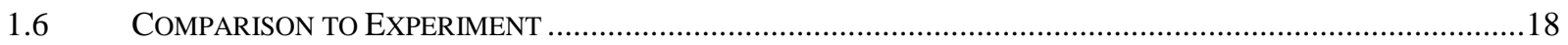

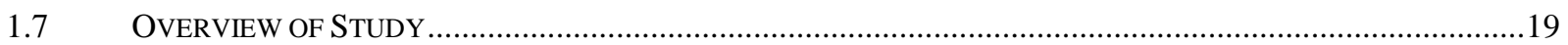

CHAPTER 2 THE KIRCHHOFF METHOD _.........................................................................................................20

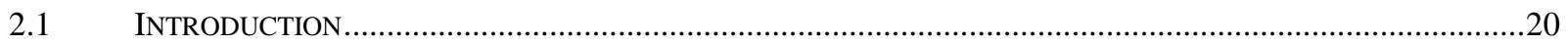

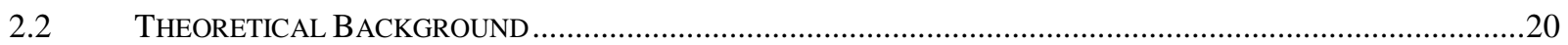

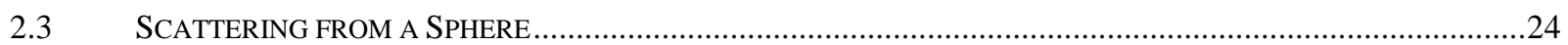

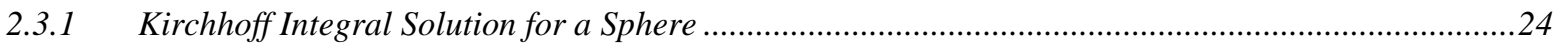

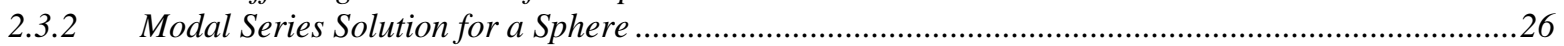

2.3.3 Comparison of Kirchhoff Method and Modal Series Solution for a Rigid/Fixed Sphere.....................27

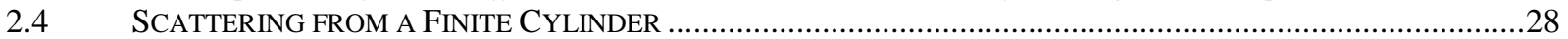

2.4.1 Kirchhoff Integral Solution for a Finite Cylinder ......................................................................29

2.4.2 Modal Series Based Solution for a Finite Cylinder ........................................................................... 31

2.4.3 Comparison of Kirchhoff Method and Modal Series Based Solution for a Rigid/Fixed Finite Cylinder 32

2.5 KIRCHHOFF METHOD APPROXIMATIONS FOR THE SAND DOLLAR ………...........................................33

2.5.1 Kirchhoff Integral Solution for an Infinitely Thin Disk ...................................................................

2.5.2 Kirchhoff Integral Solution for a Disk with Finite Thickness ..........................................................35

2.5.3 Kirchhoff Integral Solution for a Spherical Cap.............................................................................38

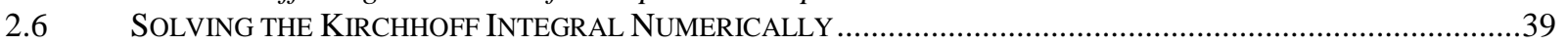

2.6.1 Creating Geometry and a Mesh in COMSOL Multiphysics..........................................................40

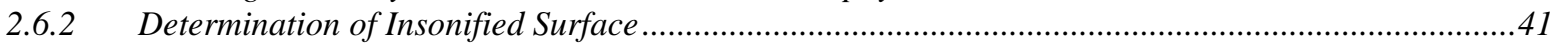

2.6.3 Evaluating the Kirchhoff Integral Numerically .................................................................................43

2.7 COMPARISON OF METHODS FOR SOLVING THE KIRCHHOFF INTEGRAL ….............................................44

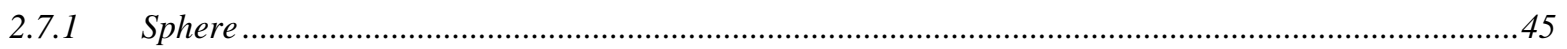

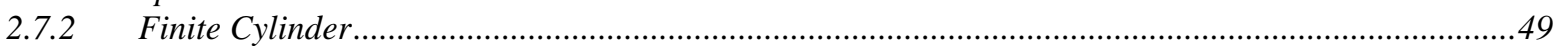

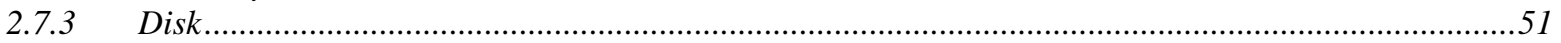

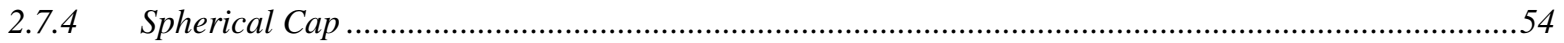

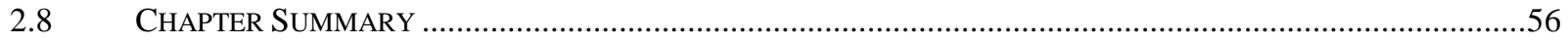

CHAPTER 3 THE FINITE ELEMENT METHOD _...............................................................................................58

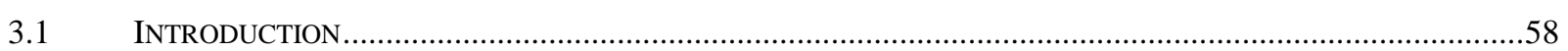

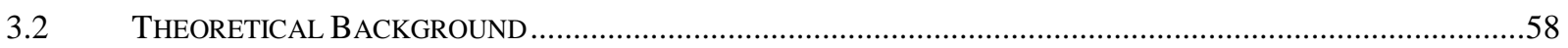

3.3 TECHNIQUE FOR REDUCING NUMERICAL ERROR IN TWO AND THREE DIMENSIONS................................66

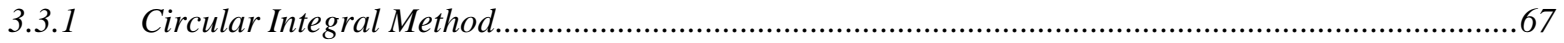

3.3.2 Spherical Integral Method ………....................................................................................... 70

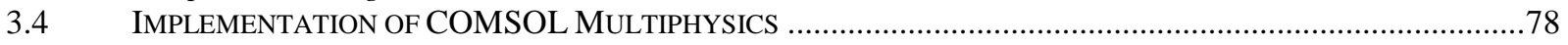

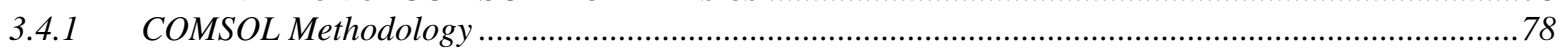

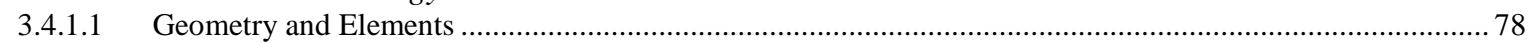

3.4.1.2 Defining the Partial Differential Equations ....................................................................................... 79

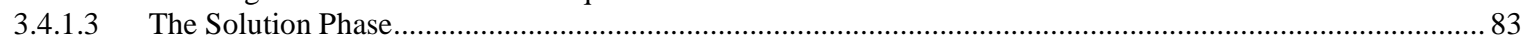

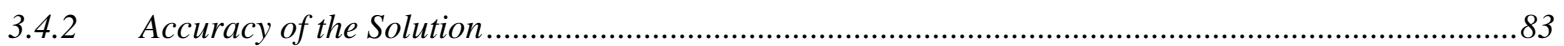

3.4.2.1 Two Dimensional Test: Rigid/Fixed Infinite Cylinder (Max Error Norm) ...............................................8 84

3.4.2.2 Two Dimensional Test: Rigid/Fixed Infinite Cylinder (Infinite Form Function) ..................................... 88

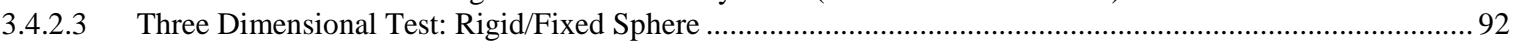

3.4.3 Methodology for Sand Dollar Predictions .................................................................................. 94 


\section{CHAPTER 4 MODEL RESULTS AND COMPARISON TO LABORATORY EXPERIMENTAL DATA 96}

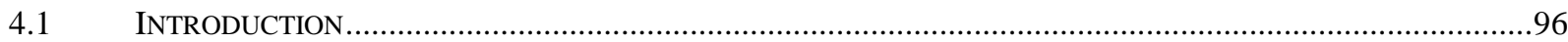

4.2 COMPARISON OF PREDICTED SCATTERING BASED ON THE KIRCHHOFF AND FINITE ELEMENT METHODS

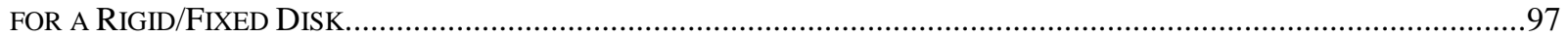

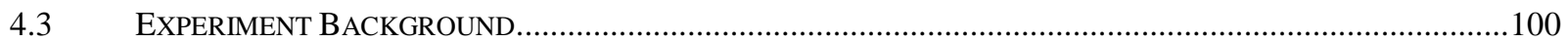

4.4 COMPARISONS OF ACOUSTIC SCATTERING PREDICTIONS TO EXPERIMENTAL DATA ..............................102

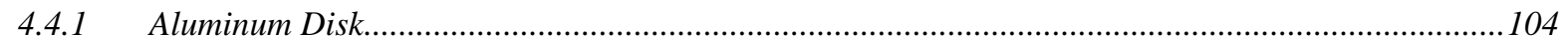

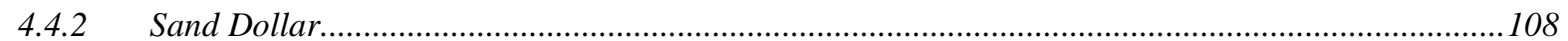

4.4.2.1 Surface Mesh Geometry from CT Scans ........................................................................................ 109

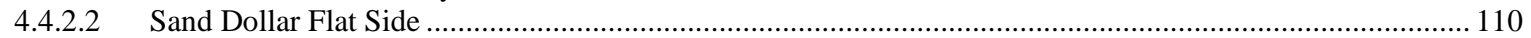

4.4.2.3 Sand Dollar Round Side ................................................................................................................ 115

4.5 HEURISTIC IMPROVEMENT TO SCATTERING MODELS DEVELOPED FOR RIGID/FIXED OBJECTS ................120

4.5.1 Reflection Coefficients from Infinite Half-Spaces and Layers .......................................................120

4.5.2 Determination of Reflection Coefficient for Aluminum Disk and Sand Dollar................................123

4.5.3 Heuristic Correction to the Rigid/Fixed Scattering Models .........................................................126

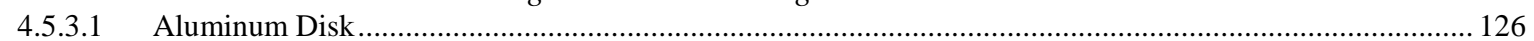

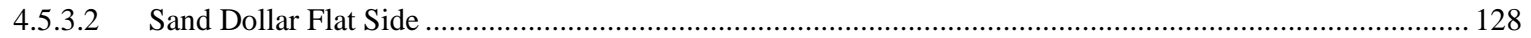

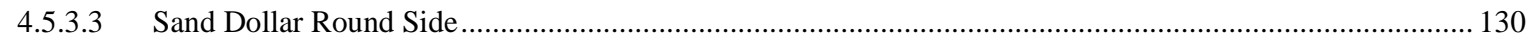

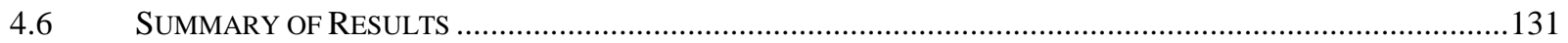

CHAPTER 5 SUMMARY AND CONCLUSION ................................................................................................133

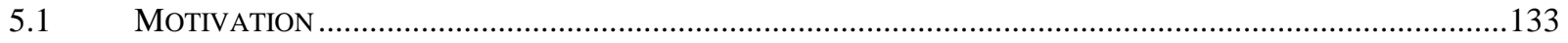

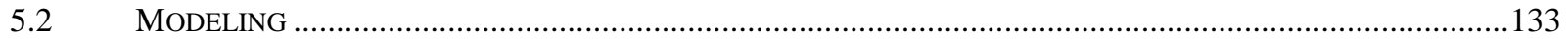

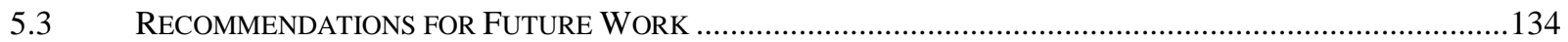

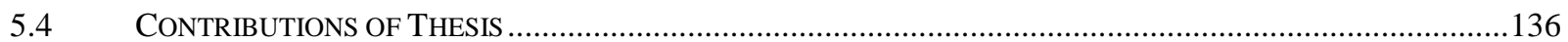




\section{List of Figures}

Figure 1 - Photograph of sand dollar test, top-view (Stanton and Chu, 2004) .............................14

Figure 2 - Spherical coordinate system, shown with incoming wave and without scatterer

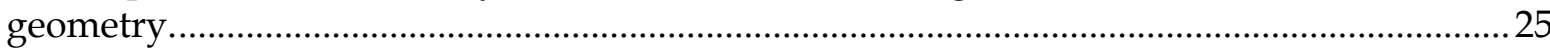

Figure 3 - Target strength of a rigid/fixed sphere of radius $5 \mathrm{~cm}$, from the Kirchhoff method

(starred line) and modal series solution (smooth line)..........................................................27

Figure 4 - Cylindrical coordinate system, shown with incoming wave and without scatterer

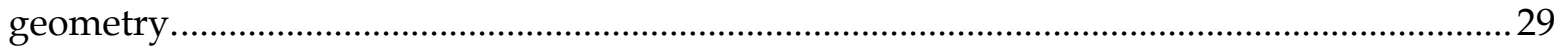

Figure 5 - Broadside target strength of a rigid/fixed finite cylinder of length $10 \mathrm{~cm}$ and radius 1

cm using the Kirchhoff method (starred line) and modal series approximation (solid line).

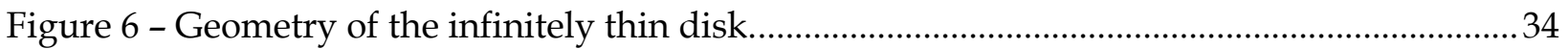

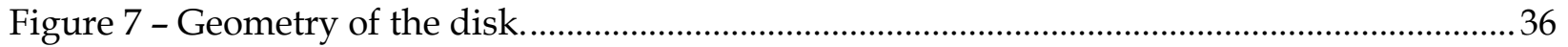

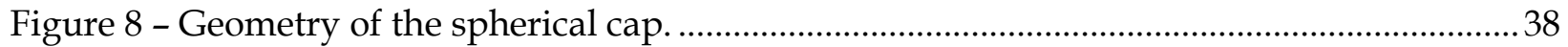

Figure 9 - Examples of minmax tests in $\mathrm{x}$ for Newell's algorithm. .............................................. 43

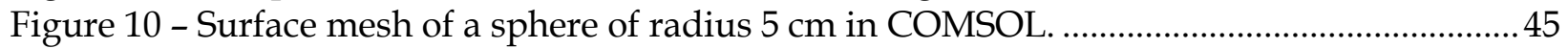

Figure 11 - Surface mesh of a sphere in MATLAB after Newell's algorithm. ..............................46

Figure 12 - Surface mesh of a sphere in MATLAB after Newell's algorithm, with triangle

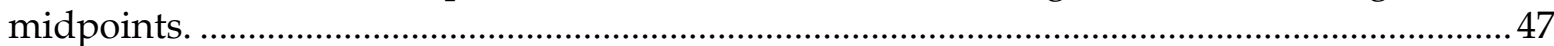

Figure 13 - Close view of surface mesh of sphere, with midpoints. ........................................... 47

Figure 14 - Target strength of a rigid/fixed sphere with radius $5 \mathrm{~cm}$ by solving the Kirchhoff

integral analytically (solid line) and numerically (stars) ........................................................ 48

Figure 15 - Surface mesh of a finite cylinder in COMSOL. .......................................................... 49

Figure 16 - Surface mesh of a finite cylinder in MATLAB after Newell's algorithm. ...................50

Figure 17 - Target strength of a rigid/fixed finite cylinder with length $10 \mathrm{~cm}$ and radius $1 \mathrm{~cm}$ by

solving the Kirchhoff integral analytically (solid line) and numerically (stars). ..................51

Figure 18 - Surface mesh of a disk in COMSOL. ........................................................................... 52

Figure 19 - Surface mesh of a disk in MATLAB after Newell's algorithm. .................................52

Figure 20 - Target strength of a rigid/fixed disk with radius $5 \mathrm{~cm}$ and thickness $1 \mathrm{~cm}$ by solving

the Kirchhoff integral analytically (solid line) and numerically (stars)................................ 53

Figure 21 - Surface mesh of a spherical cap in COMSOL............................................................ 54

Figure 22 - Surface mesh of a spherical cap in MATLAB after Newell's algorithm.....................55

Figure 23 - Target strength of a rigid/fixed spherical cap with radius $5 \mathrm{~cm}$ and radius $1.34 \mathrm{~cm}$

by solving the Kirchhoff integral analytically (solid line) and numerically (stars)...............56

Figure 24 - Domain of three dimensional scattering problem, shown with incident wave. ........59

Figure 25 - Geometry of a bounded three dimensional scattering problem................................. 60

Figure 26 - Examples of two linear (left) and three quadratic (right) triangular finite elements,

used to discretize two dimensional problems. The black dots represent the positions of the

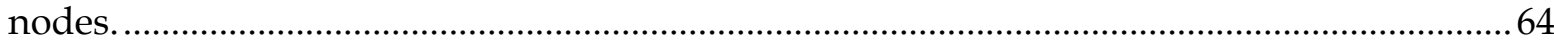

Figure 27 - Example of a three dimensional quadrilateral quadratic finite element. The black dots represent the positions of the ten nodes.

Figure 28 - Sample three dimensional and two dimensional finite element domains in COMSOL.

Figure 29 - Total and scattered pressure fields from a rigid/fixed infinite cylinder, $\mathrm{ka}=10$ in

COMSOL. The incident wave arrives from the left side of the domain. 
Figure 30 - Max error norm versus EPW for COMSOL approximation of scattering from a rigid/fixed infinite cylinder at $\mathrm{ka}=1$.

Figure 31 - Max error norm versus EPW for COMSOL approximation of scattering from a rigid/fixed infinite cylinder at $\mathrm{ka}=5$.

Figure 32 - Absolute value of infinite form function of a rigid/fixed infinite cylinder, using the modal series solution and COMSOL (point method). EPW $=6$ and SMD $=50$.

Figure 33 - Absolute value of infinite form function of a rigid/fixed infinite cylinder, using the modal series solution and COMSOL (circular integral method). EPW $=6$ and SMD $=50 \ldots 90$

Figure 34 - Error in the infinite form function approximation of a rigid/fixed infinite cylinder at $\mathrm{SMD}=50$.

Figure 35 - Error in the infinite form function approximation of a rigid/fixed infinite cylinder at $\mathrm{EPW}=6$.

Figure 36 - Target strength of a rigid/fixed sphere, radius $5 \mathrm{~cm}$, using the modal series solution and COMSOL (spherical integral method). EPW $=6$ and SMD $=20$.

Figure 37 - Target strength at broadside incidence of a rigid/fixed disk of radius $3.625 \mathrm{~cm}$ and thickness $5.5 \mathrm{~mm}$, based on (1) the analytic solution of the Kirchhoff integral (dashed line) and (2) the finite element method using COMSOL with axial symmetry (line with $x^{\prime} s$ ). EPW $=6$ and $\mathrm{SMD}=50$.

Figure 38 - Target strength at $70 \mathrm{kHz}$ of a rigid/fixed disk of radius $3.625 \mathrm{~cm}$ and thickness 5.5 $\mathrm{mm}$ based on (1) the analytic solution of the Kirchhoff integral (dashed line) and (2) the finite element method using COMSOL (line with $x^{\prime}$ s). EPW $=6$ and SMD $=20$.

Figure 39 - Cross section of a sand dollar (Dendraster excentricus) obtained from CT scans.

Details of the inner structure are revealed in addition to the high-resolution measurements of the outer shape (from Andone Lavery, personal communication).

Figure 40 - Comparison of measured target strength at $70 \mathrm{kHz}$ as a function of angle of orientation for an aluminum disk (solid starred line), radius $4 \mathrm{~cm}$ and thickness $1.9 \mathrm{~mm}$, to predicted target strength of a rigid/fixed disk of similar dimensions found by solving the Kirchhoff integral analytically (dashed line). 105

Figure 41 - Comparison of measured target strength at $70 \mathrm{kHz}$ as a function of angle of orientation for an aluminum disk (solid starred line), radius $4 \mathrm{~cm}$ and thickness $1.9 \mathrm{~mm}$, to predicted target strength of a rigid/fixed disk of similar dimensions using the finite element method in COMSOL (thin solid line with $x^{\prime}$ ). . EPW $=6$ and SMD $=20$.

Figure 42 - Three dimensional sand dollar model in COMSOL, with the round side visible....109

Figure 43 - Sand dollar surface mesh in MATLAB after implementing Newell's algorithm, with the orientation corresponding to an incident wave normal to the flat side.

Figure 44 - Comparison of predicted target strength at $70 \mathrm{kHz}$ as a function of angle of orientation for a rigid/fixed disk, radius $3.625 \mathrm{~cm}$ and thickness $5.5 \mathrm{~mm}$, based on the analytic solution of the Kirchhoff integral (dashed line) to predicted target strength of the flat side of a rigid/fixed sand dollar of similar dimensions based on a numerical solution to the Kirchhoff integral (solid line with circles).

Figure 45 - Comparison of measured target strength at $70 \mathrm{kHz}$ as a function of angle of orientation for the flat side of a sand dollar (solid starred line), to predicted target strength of a rigid/fixed disk, radius $3.625 \mathrm{~cm}$ and thickness $5.5 \mathrm{~mm}$, found by solving the Kirchhoff integral analytically (dashed line).

Figure 46 - Comparison of measured target strength at $70 \mathrm{kHz}$ as a function of angle of orientation for the flat side of a sand dollar (solid starred line), to predicted target strength 
of the flat side of a rigid/fixed sand dollar found by solving the Kirchhoff integral numerically (solid line with circles).

Figure 47 - Comparison of measured target strength at $70 \mathrm{kHz}$ as a function of angle of orientation for the flat side of a sand dollar (solid starred line), to predicted target strength of a rigid/fixed disk of radius $3.625 \mathrm{~cm}$ and thickness $5.5 \mathrm{~mm}$ using the finite element method in COMSOL (thin solid line with $x^{\prime}$ s). EPW $=6$ and SMD $=20$.

Figure 48 - Sand dollar surface mesh in MATLAB after implementing Newell's algorithm, with the orientation corresponding to an incident wave normal to the round side.

Figure 49 - Comparison of predicted target strength at $70 \mathrm{kHz}$ as a function of angle of orientation for an spherical cap, radius $3.625 \mathrm{~cm}$ and thickness $1.1 \mathrm{~cm}$, based on the analytic solution of the Kirchhoff integral (dashed line) to predicted target strength of the round side of a rigid/fixed sand dollar of similar dimensions based on a numerical solution to the Kirchhoff integral (solid line with circles).

Figure 50 - Comparison of measured target strength at $70 \mathrm{kHz}$ as a function of angle of orientation for the round side of a sand dollar (solid starred line), to predicted target strength of a rigid/fixed spherical cap, radius $3.625 \mathrm{~cm}$ and thickness $1.1 \mathrm{~cm}$, found by solving the Kirchhoff integral analytically (dashed line).

Figure 51 - Comparison of measured target strength at $70 \mathrm{kHz}$ as a function of angle of orientation for the round side of a sand dollar (solid starred line), to predicted target strength of the round side of a rigid/fixed sand dollar found by solving the Kirchhoff integral numerically (solid line with circles).

Figure 52 - Geometry of a single layer in between two infinite half spaces. ..............................121

Figure 53 - Reflection coefficients from three layer model (calcite-water-calcite). Calcite thickness refers to both top and bottom layer thickness.

Figure 54 - Comparison of measured target strength at $70 \mathrm{kHz}$ as a function of angle of orientation for an aluminum disk (solid starred line), radius $4 \mathrm{~cm}$ and thickness $1.9 \mathrm{~mm}$, to predicted target strength of a penetrable disk $\left(\left|R_{L}\right|=0.5954\right)$ of similar dimensions found by solving the Kirchhoff integral analytically (dashed line). 126

Figure 55 - Comparison of measured target strength at $70 \mathrm{kHz}$ as a function of angle of orientation for an aluminum disk (solid starred line), radius $4 \mathrm{~cm}$ and thickness $1.9 \mathrm{~mm}$, to predicted target strength of an penetrable disk $\left(\left|R_{L}\right|=0.5954\right)$ of similar dimensions using the finite element method in COMSOL (thin solid line with $x^{\prime}$ s). EPW $=6$ and SMD $=20.127$

Figure 56 - Comparison of measured target strength at $70 \mathrm{kHz}$ as a function of angle of orientation for the flat side of a sand dollar (solid starred line), to predicted target strength of the flat side of penetrable sand dollar $\left(\left|R_{L}\right|=0.4\right)$ found by solving the Kirchhoff integral numerically (solid line with circles)....

Figure 57 - Comparison of measured target strength at $70 \mathrm{kHz}$ as a function of angle of orientation for the flat side of a sand dollar (solid starred line), to predicted target strength of a penetrable disk $\left(\left|R_{L}\right|=0.4\right)$ of radius $3.625 \mathrm{~cm}$ and thickness $5.5 \mathrm{~mm}$ using the finite element method in COMSOL (thin solid line with $x^{\prime}$ s). EPW $=6$ and SMD $=20$.

Figure 58 - Comparison of measured target strength at $70 \mathrm{kHz}$ as a function of angle of orientation for the round side of a sand dollar (solid starred line), to predicted target strength of the round side of a penetrable sand dollar $\left(\left|R_{L}\right|=0.4\right)$ found by solving the Kirchhoff integral numerically (solid line with circles). 


\section{List of Tables}

Table 1 - Target information for the acoustic scattering experiment performed by Stanton and

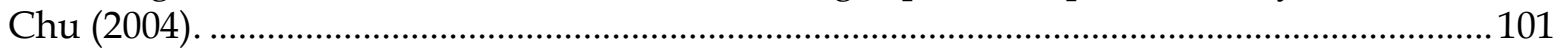

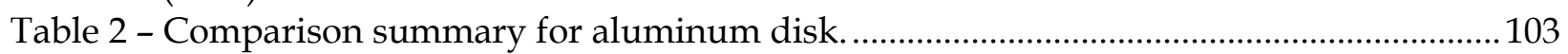

Table 3 - Comparison summary for sand dollar, flat side.......................................................103

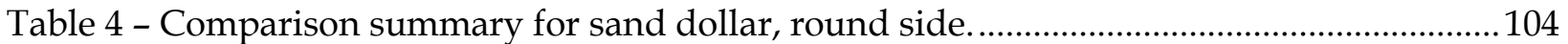

Table 5 - Reflection coefficient calculation for aluminum disk................................................. 124 


\section{Chapter 1 Introduction}

\subsection{Sound as a Tool in the Ocean}

Sound is an important tool for gaining insight into phenomena and processes in underwater environments. In the ocean, the theory of sound is essential in such broad applications as military operations, geological exploration, and biological surveys. Unlike electromagnetic waves, acoustic pressure waves are able to propagate long distances in water. The typical frequency range used in underwater acoustics is $10 \mathrm{~Hz}$ to $1 \mathrm{MHz}$, with the lower frequencies able to travel many kilometers. An important aspect of the field is acoustic scattering, that is, understanding how sound reacts to boundaries and obstacles in its path. With SONAR (Sound navigation and ranging) - a method for marine vessels to navigate, communicate, and detect one another - as its most well-known application, acoustic scattering includes the study of the reflection, diffraction, and transmission of sound incident upon a particular object. This analysis can often convey detailed information about the nature of the object such as its shape, size, or material properties. Knowledge of scattering mechanisms is important in such diverse applications as mine detection and investigating zooplankton populations. There is a vast literature on the subject of underwater acoustic scattering (Urick, 1983; Medwin and Clay, 1998). 


\subsection{Scattering from Benthic Shells}

The seafloor is a significant scatterer of sound. Not only essential to the determination of bathymetry, knowledge of how sound scatterers from the ocean bottom has commercial, military, and scientific ramifications. Sediment type, bottom roughness, and grazing angle all are important variables in seafloor scattering. Jackson et al. (1986) presented data showing how sediment type alone is not a good predictor of scattering strength, with variations up to $15 \mathrm{~dB}$ within a single class of seafloor material. Jackson and colleagues also showed how seafloor roughness seemed to account for high variability in the acoustic returns at shallow grazing angles. An important instance of seafloor roughness is the presence of benthic shelled organisms. Studies have suggested that the presence of benthic shells can contribute greatly to the scattering strength of the ocean bottom at frequencies above $10 \mathrm{kHz}$ (Stanic et al., 1989; Fenstermacher et al., 2001). Not only can shells greatly increase the scattering strength in the backwards direction, but they also scatter sound into the seafloor itself. This raises the possibility of detecting buried objects at subcritical angles (Stanton and Chu, 2004). However, the understanding of benthic shell aggregate scattering remains limited due to an incomplete knowledge of the scattering from individual benthic shells.

Accurately describing the scattering from most marine organisms is a significant challenge, in part due to their geometric complexities. In particular, there are no exact analytic expressions for the scattering from irregular benthic shells. There are only eleven relatively simple geometric shapes with separable coordinate systems, including spheres and infinite cylinders, and thus with exact analytic solutions to their scattering (Bowman et al., 1969; Anderson, 1950; Faran, 1951). In addition to geometry, material properties are also an important factor in the scattering physics of an object. These material properties are important 
in determining proper boundary conditions on the surface of the object. Fluid objects simply require continuity of pressure and velocity on their boundary. Solid objects, referred to as elastic, involve more complicated boundary conditions due to the presence of shear waves. Accurately representing the boundary conditions of an object can be just as important as its geometry in the modeling of its scattering.

There has been much work done regarding the scattering of individual zooplankton, including shelled organisms, in the water column (Simmonds and MacLennan, 2005). Certain animal classes have been approximated as simple geometrical shapes, such as spheres (Greenlaw, 1977; Johnson, 1977; Stanton et al., 1987; 2000) and finite cylinders (Stanton, 1989; Stanton et al., 1993). More complex representations of certain water-column scatterers have also been used. In particular, scattering models based on the distorted wave Born approximation (DWBA) have proved useful in modeling fluid-like zooplankton (Chu et al., 1993; Stanton et al., 1993, 1998; Stanton and Chu, 2000; Lavery et al., 2002; Lawson et al., 2006, Lavery et al., 2007).

Compared to the understanding developed for scattering from water-column organisms, relatively little work has been done regarding the scattering from individual benthic shelled organisms. Stanton et al. (2000) used a deformed sphere in both ray-based and modal-seriesbased approaches to model scattering from periwinkles (Littorina littorea), a type of benthic shelled animal. Stanton and Chu (2004) compared laboratory measurements of scattering from a machined round, elastic disk to that of a sand dollar (Dendraster excentricus) and bivalve (Dinocardium robustum vanhyningi). Specifically, they observed diffraction effects from the edges of the sand dollar through pulse-compression analysis. Though much progress was made in these studies, understanding the importance of the geometrical complexities of these benthic shelled organisms on the scattering was incomplete, which is the motivation for this study. In particular, the focus of this work is to understand acoustic scattering from sand dollars, for 
which there are controlled laboratory scattering data available (Stanton and Chu, 2004) that can give insight and inspire model development.

\subsection{Scattering from Sand Dollars}

Sand dollars (Dendraster excentricus) were shown by Fenstermacher et al. (2001) to contribute significantly to seafloor scattering levels when in naturally occurring dense collections. These benthic echinoderms can form concentrations of up to several hundred per square meter in the sandy, shallow water of the west coast of North America (Chia, 1969; Highsmith, 1982). Sand dollars have skeletons composed of strong calcite, correctly referred to as tests and not shells because of a small amount of external living tissue (Nichols, 1969). These tests, clearly visible after the animal has died, resemble thin round disks, as seen in figure 1.

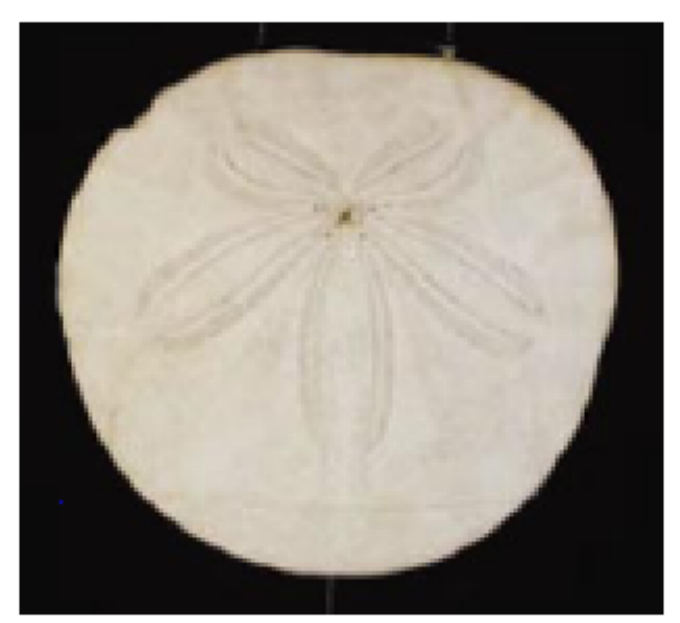

Figure 1 - Photograph of sand dollar test, top-view (Stanton and Chu, 2004).

Sand dollars grow to several centimeters in diameter; Chia's (1969) population records show an average adult diameter of $7 \mathrm{~cm}$. Understanding the scattering from individual sand dollars is 
important for predicting the scattering from aggregations of live organisms found in situ on the ocean bottom.

This work attempts to further understand the scattering from individual sand dollars by first modeling them as high aspect ratio oblate objects, namely disks and spherical caps. Along with the geometrical complexities, the material properties of sand dollars are important to an accurate description of their scattering. One key simplification to the models developed here is that a rigid/fixed, rather than elastic, boundary condition is used. This removes the complexities of shear waves and internal structure from the analysis and proves to be a good approximation at certain angles of orientation with an added heuristic reflection coefficient. Though the complex scattering mechanisms of elastic disks have been illustrated by Hefner and Marston (2001), they are beyond the scope of this work. Also, the models' focus is solely on the backscattering of incident harmonic plane waves. Both an approximate analytical technique, namely the Kirchhoff method, and a fully numerical technique, namely the finite element method, are used to model the scattering from sand dollars. Both techniques model them as simple high aspect ratio oblate objects (disks and spherical caps). The Kirchhoff method is then taken further to model the scattering from the sand dollar's exact geometry, obtained from high computed tomography (CT) scans. All work involves free field scattering, that is, the scatterer is away from all boundaries.

\subsection{The Kirchhoff Method}

The Kirchhoff method is an approximation of the Helmholtz-Kirchhoff integral, derived from the theorems of Gauss and Green. The result of the method is an analytic integral that is evaluated over the insonified surface of the scatterer to determine its reflection. There is a vast 
literature on the subject in both optics and acoustics (Born and Wolf, 1970; Medwin and Clay, 1998). Because any scattering process often also involves strong diffraction effects, there are limitations to the accuracy of the method. In particular, it loses accuracy as wavelength increases beyond the characteristic dimensions of the object. Norton et al. (1993) studied the diffraction-induced errors of this method in the scattering from circular disks.

In Chapter 2, a brief derivation of the Kirchhoff method is discussed. The resulting integral expression is then solved for two objects: a sphere and a finite cylinder. The results are compared to the sphere's exact modal series solution and the finite cylinder's modal-seriesbased approximation. Next, the Kirchhoff integral expression is solved for a disk and spherical cap, the two objects that approximate the shape of each side of the sand dollar. Finally, a method for solving the Kirchhoff method's integral numerically is presented. It is based on creating a mesh conforming to the surface of the scatterer and summing the integral over the resulting elements. This approach is valuable because it does not require the object's surface to be separable and thus can be used on arbitrarily complex scatterers. Finally, the numerical approach is tested against the previous analytic solutions of the Kirchhoff integral for the sphere, finite cylinder, disk, and spherical cap.

\subsection{The Finite Element Method}

Broadly defined, a numerical technique is a way of approximating continuous problems with discrete solutions. The idea of numerical analysis arose long before computers, evidenced by very old techniques such as Newton's and Euler's methods. However, the surge in computing power over the past several decades has greatly expanded the scope and power of using numerical techniques for solving problems. In the context of acoustic scattering, a few 
distinct numerical techniques have risen in popularity, such as the T-matrix and finite element methods. The T-matrix method is a formally exact numerical solution to the wave equation. Introduced by Waterman (1969), this approach has been used to study the scattering and diffraction of thin circular disks (Kristensson and Waterman, 1982; Norton et al., 1993; Stanton et al., 2007).

An approach to approximating solutions to partial differential equations and integral equations, the finite element method is a general numerical technique with a broad scope of applications. It stemmed from the work of applied mathematicians, physicians, and engineers in the mid twentieth century; each community was interested in approximating solutions to increasingly complex continuous problems (Huebner, 1975). The first mention of the term "finite element method" was by Clough (1960) to describe plane elasticity. Earlier, Courant (1943) used piecewise continuous functions over triangular elements to study the St. Venant torsion problem. The mathematical foundations of the finite element method were solidified in the early 1970s (Strang and Fix, 1973; Babuska and Aziz, 1973), and it has become a popular numerical technique in a wide variety of fields. Early use of the finite element method in the study of acoustics focused on internal problems such as acoustics of an enclosure, structural vibration, and waveguides (Gladwell, 1966; Craggs, 1972; Nefske et al., 1982; Petyt, et al., 1976). As techniques have been developed to deal with the infinite domain of scattering problems, the finite element method has been adapted for them as well (Hunt et al., 1975; Bettess, 1977; Gan et al., 1993; Berenger, 1994; Thompson, 2006).

In Chapter 3, the theory behind the finite element method and its application to acoustic scattering problems are discussed. Techniques for reducing numerical error in two and three dimensions are presented. The program COMSOL Multiphysics ${ }^{\circledR}$ is then explored for its use in predicting the scattering from sand dollars. The accuracy and limitations of this method are 
explored by again solving problems with known solutions, scattering from a rigid/fixed infinite cylinder and a rigid/fixed sphere.

\subsection{Comparison to Experiment}

In Chapter 4, the results from both the Kirchhoff method and finite element method models are compared with published (Stanton and Chu, 2004) experimental results. In Stanton and Chu (2004), forward scattering and backscattering from a sand dollar test, a bivalve shell, and a machined aluminum disk of similar size were measured over a range of angles of orientation. Model results based on the finite element method and analytic and numerical solutions to the Kirchhoff method are compared to the experimental results from the aluminum disk and the two faces of the sand dollar. These comparisons are conducted at a single frequency, $70 \mathrm{kHz}$, over a range of angles of incidence.

As the wavelength used is similar in size to the dimensions of the sand dollar and aluminum disk, certain inaccuracies show up in the use of the Kirchhoff method, as mentioned before. However, near normal incidence to the main faces of the sand dollar and aluminum disk (referred to as broadside), this approach results in good agreement between the predicted and measured scattering. The finite element method is able to model some of the experimental data at higher angles of orientation in addition to working well near broadside. Errors due to the use of a rigid/fixed boundary condition are discussed and a heuristic approach to representing the true penetrable condition of the scatterers by means of an added reflection coefficient is developed. 


\subsection{Overview of Study}

To summarize, Chapter 2 discusses the Kirchhoff method and the resulting solutions for two shapes similar to a sand dollar. Chapter 3 discusses the finite element method and its application in modeling the scattering by a sand dollar. Chapter 4 compares the results of the models with experimental scattering data for an aluminum disk and sand dollar. A heuristic correction to the models is discussed as well to account for the use of the rigid/fixed rather than elastic boundary conditions. Chapter 5 summarizes the thesis and offers recommendations for future work. 


\section{Chapter 2 The Kirchhoff Method}

\subsection{Introduction}

In this chapter, models for understanding the backscattering from individual sand dollars are created through use of the Kirchhoff method. A brief overview of the Kirchhoff method is presented, which results in an analytic integral, referred to as the Kirchhoff integral, which approximates the backscattering amplitude of an object when exposed to an incident harmonic plane wave. The Kirchhoff integral is tested for two shapes with known solutions, namely spheres and finite cylinders. However, because sand dollars have complicated shapes, for which there are no analytic solutions to the Kirchhoff integral, they are represented in this chapter by objects with simpler geometries that incorporate the sand dollar's high aspect ratio oblate shape, namely round disks and spherical caps. These objects are all rigid/fixed, based on the assumption that this is a good approximation of the sand dollar's boundary condition. Finally, a technique for solving the integral numerically for any shape, regardless of geometric complexities, is presented. Numerical solutions to the Kirchhoff integral are then compared to the analytic solutions for a sphere, finite cylinder, disk, and spherical cap.

\subsection{Theoretical Background}

The Kirchhoff method's derivation begins with the theory of wave propagation proposed by Christian Huygens. Described in detail by Born and Wolf (1970), Huygens' principle is that each point on an advancing wave front can be considered a source of secondary 
spherical wavelets. Together, these wavelets form the new wave front as it propagates. Using the theorems of Gauss and Green, analytic expressions for these wavelets can be written. In scattering problems, these wavelets are analyzed on the surface of the scatterer, resulting in the scattered pressure field. Medwin and Clay (1998) summarize the derivation, giving the Helmholtz-Kirchhoff integral,

$$
p_{\text {scat }}(\vec{x})=\frac{1}{4 \pi} \int_{\sigma}\left[p_{\text {scat }}\left(\vec{r}^{\prime}\right) \frac{\partial}{\partial \hat{n}}\left(\frac{e^{i k r_{\sigma}}}{r_{\sigma}}\right)-\frac{e^{i k r_{\sigma}}}{r_{\sigma}} \frac{\partial p_{s c a t}\left(\vec{r}^{\prime}\right)}{\partial \hat{n}}\right]_{\sigma} d \sigma
$$

This is an expression for the scattered pressure at position $\vec{x}$ in terms of its value along the surface of the scatterer, $\sigma$. Each differential element $d \sigma$ on the object's surface has position $\vec{r}^{\prime}$ and outward unit normal $\hat{n}$. The term $r_{\sigma}$ is the distance from the surface element to position $\vec{x}$, $r_{\sigma}=\left|\vec{x}-\vec{r}^{\prime}\right|$ and $k(=2 \pi / \lambda$, where $\lambda$ is the wavelength $)$ is the acoustic wavenumber. The Kirchhoff approximation simplifies the integral by assuming that each differential element reflects sound in a ray-like manner; each element behaves just as a part of a tangent infinite planar interface would (Medwin and Clay, 1998). This means that the scattered pressure can be written in terms of the incident pressure and the reflection coefficient $R_{I}$ that would result from such an interface. Implicit in this approximation is that only the insonified or "front" of the object contributes to scattering. The field in the shadow or "back" of the object is zero. Therefore, the integral for the scattered pressure is only evaluated on the scatter's insonified surface,

$$
p_{s c a t}(\vec{x})=\frac{1}{4 \pi} \int_{\sigma} R_{I} \frac{\partial}{\partial n}\left(p_{i n c}\left(\vec{r}^{\prime}\right) \frac{e^{i k r_{\sigma}}}{r_{\sigma}}\right) d \sigma
$$

To simplify further, the incident field is considered to be a harmonic plane wave, written in its time independent form as $p_{i n c}\left(\vec{r}^{\prime}\right)=p_{0} e^{i \vec{k} \cdot \vec{r}^{\prime}}$, with amplitude $p_{0}$ and wave vector $\vec{k}$, where 
$\vec{k}=k \hat{k}$ and $\hat{k}$ is a unit vector in the direction of the wave's propagation. (Throughout this work, only harmonic waves are considered, so the time dependence is omitted). Inserting the expression for the incident field into the integral gives

$$
p_{\text {scat }}(\vec{x})=\frac{p_{0}}{4 \pi} \int_{\sigma} R_{I} \frac{\partial}{\partial n}\left(e^{i \vec{k} \cdot \vec{r}^{\prime}} \frac{e^{i k r_{\sigma}}}{r_{\sigma}}\right) d \sigma .
$$

Two further simplifications are made. First, the scatterer is considered to have a rigid/fixed boundary condition; this is defined as one where both the gradient of pressure and the fluid velocity are zero on the boundary. The reflection coefficient for a rigid/fixed interface is always equal to one, regardless of angle of orientation. Second, the scattered pressure is only calculated at a distance from the object much larger than the object's dimensions. To do this, the coordinate system must be oriented so that the scatterer is located at the origin. Then the distance from the origin to the point $\vec{x}$, defined as $r=|\vec{x}|$, must satisfy $r>>d$, where $d$ is a typical object dimension. When this is the case, $r$ is a good approximation of the $r_{\sigma}$ term in the denominator. The $r_{\sigma}$ in the exponent must be approximated more carefully due to the importance of the phase,

$$
r_{\sigma} \approx r-\vec{r}^{\prime} \cdot \frac{\vec{x}}{r}
$$

When the scattered pressure is calculated in the backwards direction, opposite to the direction of the incident wave, this can be rewritten as,

$$
r_{\sigma} \approx r+\vec{r}^{\prime} \cdot \hat{k}
$$

With these approximations, the backscattered pressure (from equation (2.3)) can be expressed as

$$
p_{b s}(\vec{x})=\frac{p_{0}}{4 \pi} \int_{\sigma} \frac{\partial}{\partial n}\left(e^{i 2 \vec{k} \cdot \vec{r}^{\prime}} \frac{e^{i k r}}{r}\right) d \sigma .
$$


The normal derivative of $\frac{e^{i k r}}{r}$ is assumed to be zero because $r$ is large, giving

$$
\frac{\partial}{\partial n}\left(e^{i 2 \vec{k} \cdot \vec{r}^{\prime}} \frac{e^{i k r}}{r}\right) \approx i \frac{e^{i k r}}{r}(\hat{n} \cdot 2 \vec{k}) e^{i 2 \vec{k} \cdot \vec{r}^{\prime}}
$$

The integral thus reduces to

$$
p_{b s}(\vec{x})=\frac{i}{\lambda} p_{0} \frac{e^{i k r}}{r} \int_{\sigma}(\hat{n} \cdot \hat{k}) e^{i 2 \vec{k} \cdot \vec{r}^{\prime}} d \sigma .
$$

This is the final Kirchhoff method expression for the backscattered pressure.

In the far-field $k r>>1$, the scattered pressure is defined as

$$
p_{\text {scat }}=p_{0} \frac{e^{i k r}}{r} f(\Omega)
$$

where $f(\Omega)$ is known as the scattering amplitude. It is a function of the spherical angles represented by $\Omega$. Thus from equation (2.8), the backscattering amplitude, $f_{b s}$, can be written,

$$
f_{b s}=\frac{i}{\lambda} \int_{\sigma}(\hat{k} \cdot \hat{n}) e^{i 2 \vec{k} \cdot \vec{r}^{\prime}} d \sigma
$$

Backscattering amplitude can be used to find target strength (Urick, 1983),

$$
T S=10 \log \left|f_{b s}\right|^{2}
$$

The units of target strength are decibels $(\mathrm{dB})$ relative to $1 \mathrm{~m}^{2}$. Target strength is a heavily used indicator of scattering strength and is based solely on the properties of the scatterer itself. Only backscattering is considered throughout this work, so the subscript $b s$ can be dropped. This expression in equation (2.10) is referred to here as the Kirchhoff integral and can be used to calculate the scattering of an object by performing the integral over its insonified surface.

The Kirchhoff method is also referred to as the physical optics method. By only accounting for reflection from the object's front insonified surface, this method neglects the 
effects of diffraction. Diffraction effects vary greatly with frequency and object shape, making the Kirchhoff method a poor approximation at times. These effects can be explored by comparisons with shapes that have known exact solutions or approximations to their scattering, such as spheres and finite cylinders.

\subsection{Scattering from a Sphere}

In this section, the Kirchhoff integral is solved for a rigid/fixed sphere. The results are compared to the exact modal series solution for scattering from a rigid/fixed sphere.

\subsubsection{Kirchhoff Integral Solution for a Sphere}

The first step involves setting up a spherical coordinate system with radial variable $\rho$, polar variable $\theta$, and azimuth variable $\varphi$. The Cartesian coordinate system is described with unit vectors $\hat{x}, \hat{y}$, and $\hat{z}$. Assume a rigid/fixed sphere with radius $a$ is centered at the origin and consider an incident plane wave $p_{i n c}=p_{0} e^{-i k z}$ proceeding in the negative $\mathrm{z}$ direction, as seen in figure 2. This orientation is chosen to simplify the limits of integration in the integral. 


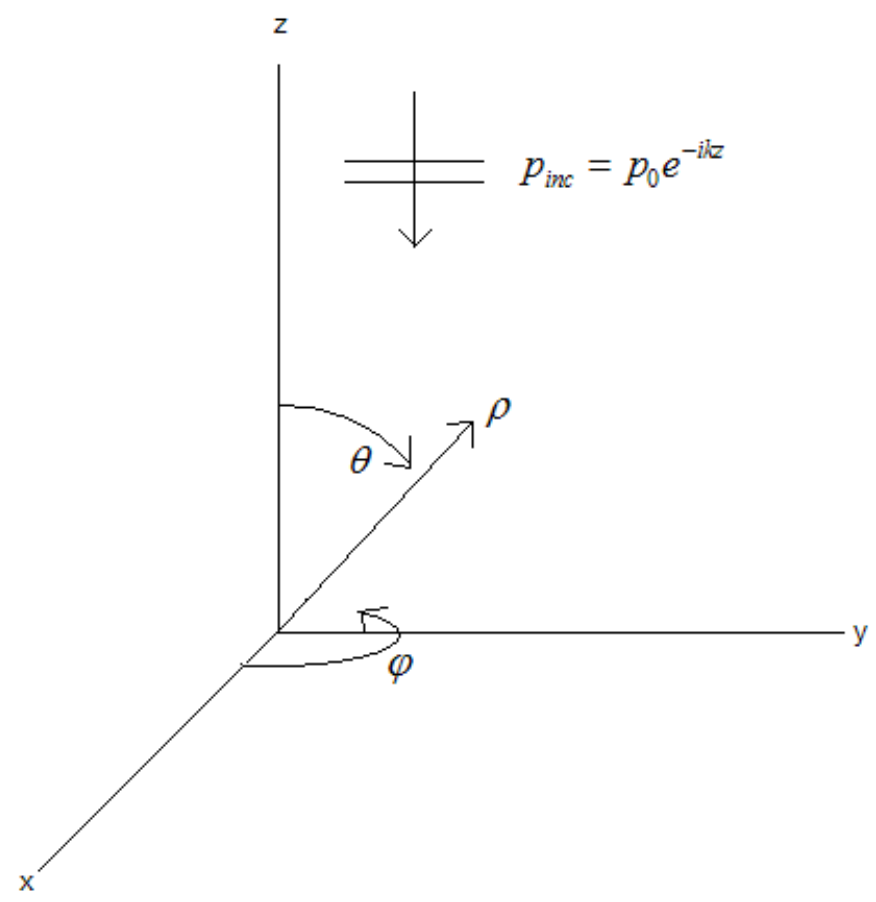

Figure 2 - Spherical coordinate system, shown with incoming wave and without scatterer geometry.

It is seen that

$$
\begin{gathered}
\vec{k}=-k \hat{z} \\
\hat{n}=\cos \varphi \sin \theta \hat{x}+\sin \varphi \sin \theta \hat{y}+\cos \theta \hat{z} \\
\vec{r}^{\prime}=a \cos \varphi \sin \theta \hat{x}+a \sin \varphi \sin \theta \hat{y}+a \cos \theta \hat{z} \\
d \sigma=a^{2} \sin \theta d \theta d \varphi .
\end{gathered}
$$

In this case, the Kirchhoff integral becomes

$$
f=\frac{i}{\lambda} \int_{0}^{2 \pi} \int_{0}^{\pi / 2}-\cos \theta e^{-i 2 k a \cos \theta} a^{2} \sin \theta d \theta d \varphi
$$

The integral over $\varphi$ is easily performed, leaving

$$
f=\frac{-i 2 \pi a^{2}}{\lambda} \int_{0}^{\pi / 2} \cos \theta e^{-i 2 k a \cos \theta} \sin \theta d \theta
$$

Making the substitution of $x=\cos \theta$, 


$$
f=-i k a^{2} \int_{-1}^{0}-x e^{-i 2 k a x} d x
$$

the expression for backscattering amplitude becomes

$$
f=\frac{i}{4 k}+\frac{a e^{-i 2 k a}}{2}-\frac{i e^{-i 2 k a}}{4 k} .
$$

Target strength follows from equation (2.11).

\subsubsection{Modal Series Solution for a Sphere}

Again consider the spherical coordinate system with a rigid/fixed sphere of radius $a$ centered at the origin and an incident plane wave $p_{i n c}=p_{0} e^{-i k z}$ traveling in the negative $\mathrm{z}$ direction. The scattered pressure from a fluid sphere is can be written as a series of modal terms:

$$
p_{s c a t}(r, \theta, \varphi)=\sum_{m=0}^{\infty} B_{m} P_{m}(\cos \theta) h_{m}^{(1)}(k r),
$$

where $P_{m}$ is the Legendre polynomial and $h_{m}^{(1)}$ is the spherical Hankel function of the first kind (Anderson, 1950). A rigid/fixed sphere may be thought of as a fluid sphere with infinite density. In this case, the coefficient $B_{m}$ becomes

$$
B_{m}=\frac{-p_{0}(-i)^{m}(2 m+1) j_{m}^{\prime}(k a)}{h_{m}^{(1)^{\prime}}(k a)}
$$

where $j_{m}$ is the spherical Bessel function of the first kind. The derivatives marked by the primes are with respect to the functions' argument, $k a$. For backscattering, $\theta=0$ and $P_{m}(\cos \theta)=1$ for all values of $m$. Simplifying, the backscattered pressure from a rigid/fixed sphere is 


$$
p_{b s}(r)=\sum_{m=0}^{\infty} \frac{-p_{0}(-i)^{m}(2 m+1) h_{m}(k r) j_{m}{ }^{\prime}(k a)}{h_{m}^{(1)^{\prime}}(k a)} .
$$

The backscattering amplitude and target strength can now be found using equations (2.10) and (2.11).

\subsubsection{Comparison of Kirchhoff Method and Modal Series Solution for a}

\section{Rigid/Fixed Sphere}

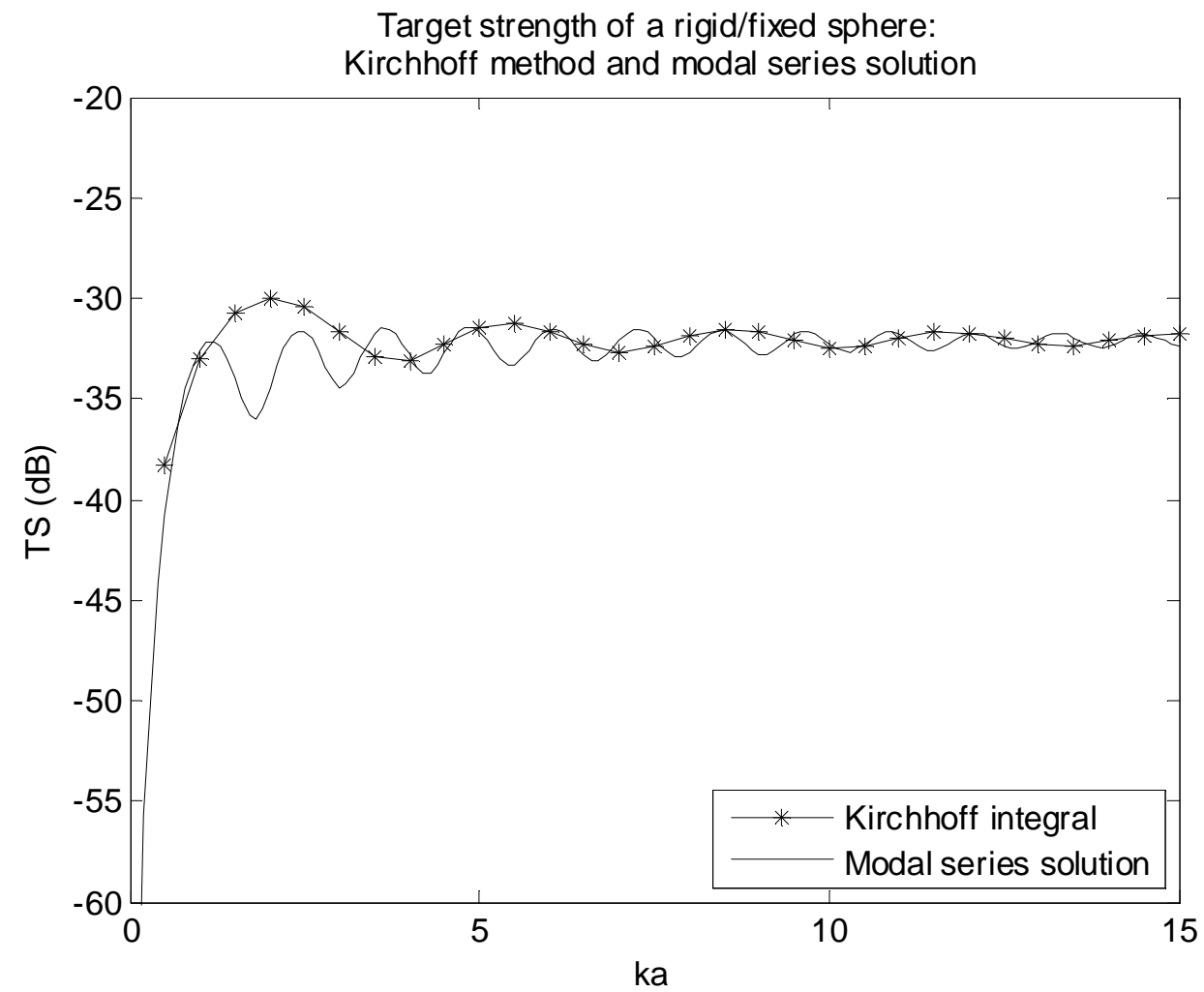

Figure 3 - Target strength of a rigid/fixed sphere of radius $5 \mathrm{~cm}$, from the Kirchhoff method (starred line) and modal series solution (smooth line).

Figure 3 shows how the Kirchhoff method result seems to converge to the exact solution as $k a$ increases. However, both curves have strikingly different phases for their peak and null 
patterns. The reason for this is that the Kirchhoff method does not include the effects of diffraction around the sphere. The modal series solution includes both the effects of reflected and diffracted, or creeping, waves. Described by Franz (1957), creeping waves diffract around the object and encircle it multiple times. Watson (1918) and Sommerfeld (1949) described the phenomenon in the study of electric waves diffracting around the Earth. Creeping waves constantly radiate off energy tangentially from the object; some of that radiates in the backwards direction and interferes with the reflected wave. They can be measured experimentally by using short sound pulses due to their increased travel distance and time (Uberall et al., 1966). These waves can also be influenced by surface waves if the object is elastic. With a rigid/fixed boundary condition, however, they only depend on the geometry of the scatterer. As $k a$ increases and these effects of diffraction lessen, the Kirchhoff method provides a better approximation to the exact modal series solution.

\subsection{Scattering from a Finite Cylinder}

In this section, the Kirchhoff integral is solved for a rigid/fixed finite cylinder. The results are compared to an approximate modal series based solution for a rigid/fixed finite cylinder. This latter approximation is based on the exact modal series solution for scattering from an infinite cylinder. The approximation neglects the effects of the end of the finite cylinder, and is therefore most accurate near broadside. Therefore, the Kirchhoff integral for the rigid/fixed finite cylinder is solved so the incident wave is normal to the cylinder's axis. 


\subsubsection{Kirchhoff Integral Solution for a Finite Cylinder}

The first step involves defining a cylindrical coordinate system with radial variable $r$, polar variable $\theta$, vertical variable $z$, and the same Cartesian coordinate system as was defined in the previous section. Assume the finite cylinder with length $L$ and radius $a$ is centered at the origin so that it is lengthwise along the $z$ axis. An incident plane $p_{i n c}=p_{0} e^{i k x}$ wave traveling in the positive $x$ direction strikes the cylinder normal to its axis. Because the incoming wave arrives at broadside, the cylinder ends are not insonified and therefore do not contribute to the scattering as calculated using the Kirchhoff integral. Therefore only the sides of the cylinder are considered.

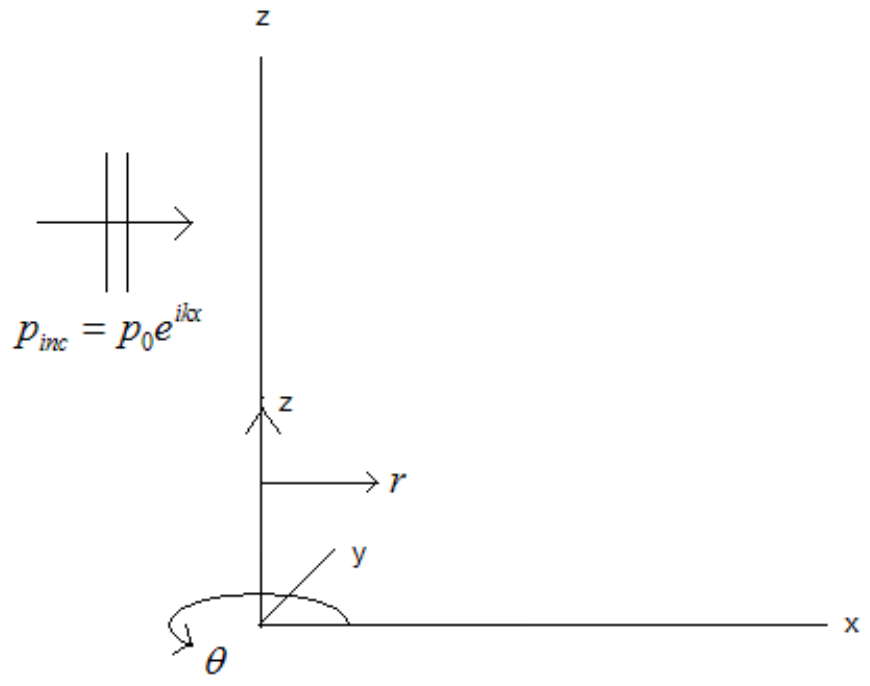

Figure 4 - Cylindrical coordinate system, shown with incoming wave and without scatterer geometry.

It can thus be seen that

$$
\begin{gathered}
\vec{k}=k \hat{x} \\
\hat{n}=\cos \theta \hat{x}+\sin \theta \hat{y}
\end{gathered}
$$




$$
\begin{gathered}
\vec{r}^{\prime}=a \cos \theta \hat{x}+a \sin \theta \hat{y}+z \theta \hat{z} \\
d \sigma=a d \theta d z .
\end{gathered}
$$

Substituting these expression into the Kirchhoff integral gives

$$
f=\frac{i a}{\lambda} \int_{-L / 2}^{L / 2} \int_{\pi / 2}^{3 \pi / 2} \cos \theta e^{i 2 k a \cos \theta} d \theta d z
$$

The integral over $z$ is straightforward, leaving

$$
f=\frac{i k a L}{2 \pi} \int_{\pi / 2}^{3 \pi / 2} \cos \theta e^{i 2 k a \cos \theta} d \theta
$$

It is split in order to help solve

$$
f=\frac{i k a L}{2 \pi}\left[\int_{\pi / 2}^{\pi} \cos \theta e^{i 2 k a \cos \theta} d \theta+\int_{\pi}^{3 \pi / 2} \cos \theta e^{i 2 k a \cos \theta} d \theta\right] .
$$

Two substitutions are made: in the first integral, $\alpha=-\theta+\pi$; in the second integral, $\beta=\theta-\pi$ :

$$
f=\frac{i k a L}{2 \pi}\left[\int_{0}^{\pi / 2}-\cos \alpha e^{-i 2 k a \cos \alpha} d \alpha+\int_{0}^{\pi / 2}-\cos \beta e^{-i 2 k a \cos \beta} d \beta\right] .
$$

Both integrals can be solved with help of the relationship (Gaunaurd, 1985)

$$
\int_{0}^{\pi / 2} \cos x e^{-i z \cos x} d x=1-\frac{\pi}{2}\left[H_{1}(z)+i J_{1}(z)\right]
$$

where $H_{1}$ is the Struve function of order one, and $J_{1}$ is the Bessel function of the first kind of order one. Thus, equation (2.24) becomes

$$
f=\frac{-i k a L}{2 \pi}\left[2-\pi\left[H_{1}(2 k a)+i J_{1}(2 k a)\right]\right] .
$$

Target strength easily follows from equation (2.11). 


\subsubsection{Modal Series Based Solution for a Finite Cylinder}

The modal series approximation for the scattering of finite cylinders is based on the exact modal series solution for an infinite cylinder. It assumes that end effects are negligible, and, therefore, it is much more accurate near broadside than at high angles of incidence. Stanton (1988) gives the expression for scattering from a rigid/fixed finite cylinder of length $L$ and radius $a$ :

$$
\begin{aligned}
& \text { where } \quad f=\frac{-i L}{\pi} \frac{\sin \Delta}{\Delta} \sum_{m=0}^{\infty} B_{m}(-i)^{m} \cos (m \varphi) \\
& \qquad \Delta=\frac{k L\left(\hat{r}_{i}-\hat{r}_{r}\right) \cdot \hat{r}_{c}}{2} \\
& \text { and } \quad B_{m}=\frac{-\varepsilon_{m} i^{m} J_{m}{ }^{\prime}(k a)}{H_{m}^{(1)^{\prime}}(k a)} .
\end{aligned}
$$

The $\hat{r}$ terms represent the unit position vectors of the source (subscript $i$ ), receiver (subscript $r$ ), and cylinder's axis (subscript c). In the case of broadside incidence, $\Delta=0$. The term $\varphi$ is the angle between the source and receiver vectors in the plane perpendicular to the cylinder axis.

For the backscattering case, $\varphi=\pi$, and therefore $\cos (m \varphi)=(-1)^{m}$. The term $\varepsilon_{m}$ is equal to 1 for $m=0$ and equal to 2 for all other vales of $m$. Thus, the backscattering amplitude becomes

$$
f=\frac{i L}{\pi} \sum_{m=0}^{\infty} \frac{\varepsilon_{m}(-1)^{m} J_{m}{ }^{\prime}(k a)}{H_{m}^{(1)^{\prime}}(k a)} .
$$

Target strength follows from equation (2.11). 


\subsubsection{Comparison of Kirchhoff Method and Modal Series Based Solution for a Rigid/Fixed Finite Cylinder}

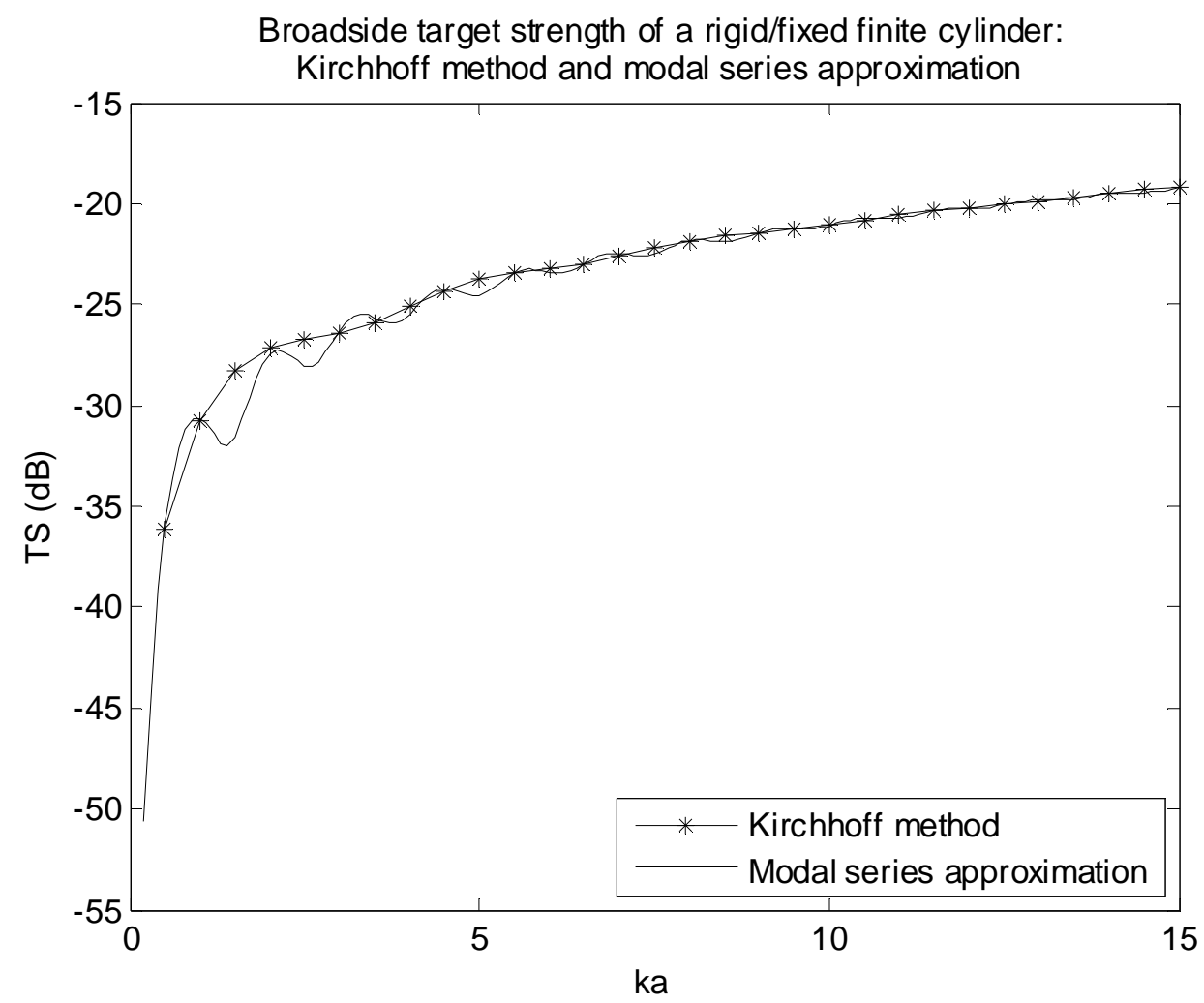

Figure 5 - Broadside target strength of a rigid/fixed finite cylinder of length $10 \mathrm{~cm}$ and radius $1 \mathrm{~cm}$ using the Kirchhoff method (starred line) and modal series approximation (solid line).

As with the sphere, there is a discrepancy in the null-peak phase patterns caused by the creeping waves not accounted for in the Kirchhoff method. The effects of this are again lessened as $k a$ increases. There seems to be much less of an effect from creeping waves for the finite cylinder than for the sphere, as evidenced by the former's better agreement with the Kirchhoff method. It is hypothesized that while the waves diffract relatively well around the entire body of the sphere and the curved sides of the cylinder, they do not diffract as well around the flat ends of the cylinder. 


\subsection{Kirchhoff Method Approximations for the Sand Dollar}

When an object's geometry becomes too complex, it may not be possible to obtain a closed form solution to the Kirchhoff integral. However, it is possible under some circumstances to approximate the complex geometry of the object with a simpler geometry that does have an analytic solution to the Kirchhoff integral. A sand dollar is an example of a complex geometry where the integral expression cannot be solved analytically due to its irregular shape and surface roughness. Generally, sand dollars have flat bottoms and slightly domed tops in addition to fine scale surface roughness. In this section, the Kirchhoff integral is solved for objects that approximate both of the orientations of the sand dollar. A round disk is used to model the flat bottom and a spherical cap is used to model the rounded top. This section starts with the use of the Kirchhoff integral to derive scattering from an infinitely thin, rigid/fixed disk, as this sheds light on the derivation for the (finite thickness) disk and spherical cap.

\subsubsection{Kirchhoff Integral Solution for an Infinitely Thin Disk}

The first step in solving the Kirchhoff integral for a disk is to start with the simplest case, a circular rigid/fixed disk of radius $a$ and zero thickness. The lack of thickness means that only the front round face of the disk is incorporated into the solution. Returning to the cylindrical coordinate system, assume the disk is located at the origin, perpendicular to the $\mathrm{z}$ axis. The incoming wave vector $\vec{k}$ forms an angle $\beta$ with the $\mathrm{z}$ axis, varying over the range of angles $\left(\frac{-\pi}{2}, \frac{\pi}{2}\right)$ 


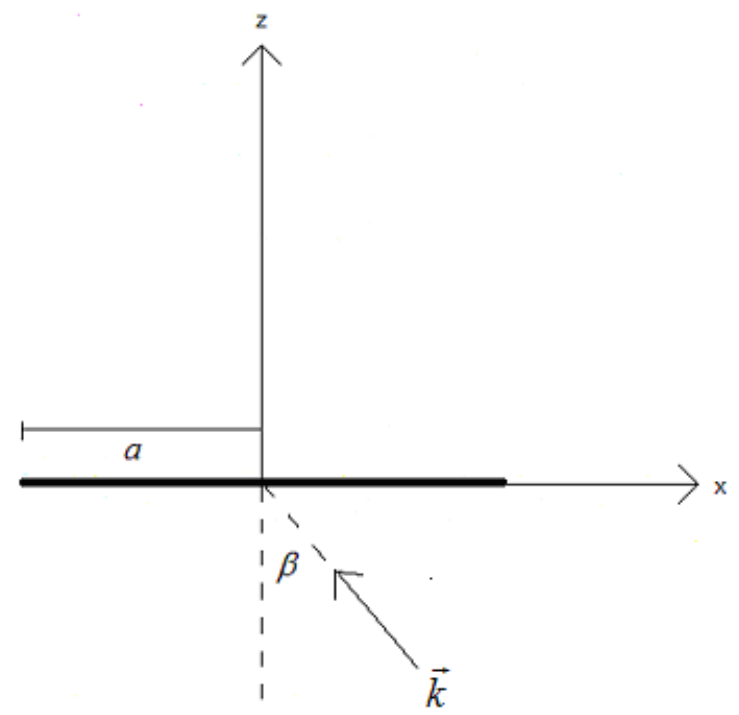

Figure 6 - Geometry of the infinitely thin disk.

In this coordinate system, the relevant parameters of the Kirchhoff integral are given by

$$
\begin{gathered}
\vec{k}=-k \sin \beta \hat{x}+k \cos \beta \hat{z} \\
\hat{n}=-\hat{z} \\
\vec{r}^{\prime}=r \cos \theta \hat{x}+r \sin \theta \hat{y} \\
d \sigma=r d r d \theta .
\end{gathered}
$$

Substituting these into the Kirchhoff integral, the backscattering amplitude is given by

$$
f=\frac{-2 i \cos \beta}{\lambda} \int_{0}^{a} r \int_{0}^{\pi} e^{-i 2 k r \sin \beta \cos \theta} d \theta d r
$$

Abramowitz and Stegun (1965) give an integral definition of a Bessel function of the first kind:

$$
J_{n}(z)=\frac{i^{n}}{\pi} \int_{0}^{\pi} e^{i z \cos \vartheta} \cos (n \vartheta) d \vartheta
$$

Substituting this expression back into equation (2.32), the backscattering amplitude is 


$$
f=\frac{-2 \pi i \cos \beta}{\lambda} \int_{0}^{a} r J_{0}(-2 k r \sin \beta) d r
$$

Next, the substitution $z=-2 k r \sin \beta$ is made

$$
f=\frac{-2 \pi i \cos \beta}{\lambda} \int_{0}^{-2 k a \sin \beta} \frac{-z}{2 k \sin \beta} J_{0}(z) \frac{-d z}{2 k \sin \beta}=\frac{-\pi i \cos \beta}{2 \lambda k^{2} \sin ^{2} \beta} \int_{0}^{-2 k a \sin \beta} z J_{0}(z) d z
$$

Abramowitz and Stegun give the derivative of a Bessel function as

$$
\left(\frac{1}{z} \frac{d}{d z}\right)^{\mu}\left[z^{v} J_{v}(z)\right]=z^{v-\mu} J_{v-\mu}(z)
$$

Setting $\mu=v=1$, this simplifies to

$$
\frac{d}{d z}\left[z J_{1}(z)\right]=z J_{0}(z)
$$

Substituting and solving,

$$
f=\frac{-\pi i \cos \beta}{2 \lambda k^{2} \sin ^{2} \beta} \int_{0}^{-2 k a \sin \beta} d\left[z J_{1}(z)\right]=\frac{i a J_{1}(-2 k a \sin \beta) \cos \beta}{2 \sin \beta}
$$

The target strength of the rigid/fixed, infinitely thin disk is thus given by

$$
T S=10 \log \left|\frac{a J_{1}(-2 k a \sin \beta) \cos \beta}{2 \sin \beta}\right|^{2}
$$

Urick (1983) gives this result in a slightly different form as the target strength of a circular plate.

\subsubsection{Kirchhoff Integral Solution for a Disk with Finite Thickness}

The next step in solving the Kirchhoff integral for the disk is to give it a thickness. There are now two different scattering regions of the disk: the flat round face and the curved edge. The height of this new edge is the disk's thickness, T. 


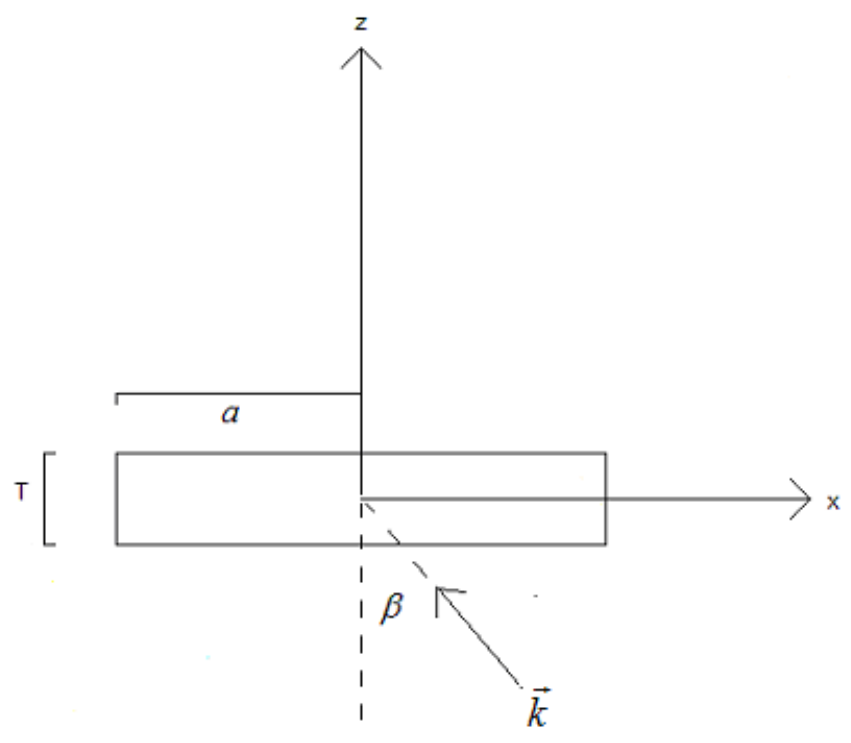

Figure 7 - Geometry of the disk.

The disk is simply a finite cylinder with an extremely high radius to length aspect ratio.

Therefore, this derivation is like that of the finite cylinder over any angle of incidence. The Kirchhoff integral can be split to analyze each of the disk's elements. These two regions of scattering add coherently to give the total scattering amplitude,

$$
f=f_{\text {face }}+f_{\text {edge }} .
$$

The backscattering amplitude of the face is very similar to before, with the only difference being a phase shift due to the face's negative displacement by $\mathrm{T} / 2$,

$$
f_{\text {face }}=\frac{i k a^{2} J_{1}(-2 k a \sin \beta) \cos \beta}{2 k a \sin \beta} e^{-i k T \cos \beta} .
$$

Next, the scattering from the edge is evaluated. The relevant parameters within the Kirchhoff integral are given by

$$
\vec{k}=-k \sin \beta \hat{x}+k \cos \beta \hat{z}
$$




$$
\begin{gathered}
\hat{n}=\cos \theta \hat{x}+\sin \theta \hat{y} \\
\vec{r}^{\prime}=a \cos \theta \hat{x}+a \sin \theta \hat{y}+z \hat{z} \\
d \sigma=a d \theta d z .
\end{gathered}
$$

Substituting into the integral expression, the backscattering amplitude is given by

$$
f_{\text {edge }}=\frac{i}{\lambda} \int_{-T / 2}^{T / 2} \int_{-\pi / 2}^{\pi / 2}-\sin \beta \cos \theta e^{i 2(-k a \sin \beta \cos \theta+k z \cos \beta)} a d \theta d z
$$

The integral over $z$ is straightforward:

$$
f_{\text {edge }}=\frac{-i a \sin (k T \cos \beta) \sin \beta}{2 \pi \cos \beta} \int_{-\pi / 2}^{\pi / 2} \cos \theta e^{-i 2 k a \sin \beta \cos \theta} d \theta
$$

To solve this integral it first must be split. Then a substitution $\alpha=-\theta$ is made in one of the resulting integrals:

$$
\begin{aligned}
& f_{\text {edge }}=\frac{-i a \sin (k T \cos \beta) \sin \beta}{2 \pi \cos \beta}\left[\int_{-\pi / 2}^{0} \cos \theta e^{-i 2 k a \sin \beta \cos \theta} d \theta+\int_{0}^{\pi / 2} \cos \theta e^{-i 2 k a \sin \beta \cos \theta} d \theta\right] \\
& f_{\text {edge }}=\frac{-i a \sin (k T \cos \beta) \sin \beta}{2 \pi \cos \beta}\left[\int_{0}^{\pi / 2} \cos \alpha e^{-i 2 k a \sin \beta \cos \alpha} d \alpha+\int_{0}^{\pi / 2} \cos \theta e^{-i 2 k a \sin \beta \cos \theta} d \theta\right] .
\end{aligned}
$$

These integrals may now be solved with help from equation (2.25), giving

$$
f_{\text {edge }}=\frac{-i a \sin (k T \cos \beta) \sin \beta}{2 \pi \cos \beta}\left[2-\pi\left[H_{1}(2 k a \sin \beta)+i J_{1}(2 k a \sin \beta)\right]\right] .
$$

Gaunaurd (1985) presents a similar derivation and arrives at the same result in his discussion of total insonification of a finite cylinder. The target strength of the rigid/fixed disk is thus given by 


$$
\begin{aligned}
T S & =10 \log \left|f_{\text {face }}+f_{\text {edge }}\right|^{2} \\
& =10 \log \left|\begin{array}{r}
\frac{i k a^{2} J_{1}(-2 k a \sin \beta) \cos \beta}{2 k a \sin \beta} e^{-i k T \cos \beta}-\left.\frac{i a \sin (k T \cos \beta) \sin \beta}{2 \pi \cos \beta}\right|^{2} \\
\times\left[2-\pi\left[H_{1}(2 k a \sin \beta)+i J_{1}(2 k a \sin \beta)\right]\right]
\end{array}\right|
\end{aligned}
$$

\subsubsection{Kirchhoff Integral Solution for a Spherical Cap}

The next object that the Kirchhoff integral is solved for is a spherical cap, modeling the sand dollar's top side. Returning to the spherical coordinate system, assume a sphere of radius $a$ centered at the origin. A slice perpendicular to the $x-y$ plane is made through the top half of the sphere, and everything below is discarded, leaving only the top cap of the sphere. Another way to define the cap is by polar angle, $\theta=0$ to some angle $\eta$. The incident wave makes an angle $\beta$ with the $\mathrm{z}$ axis.

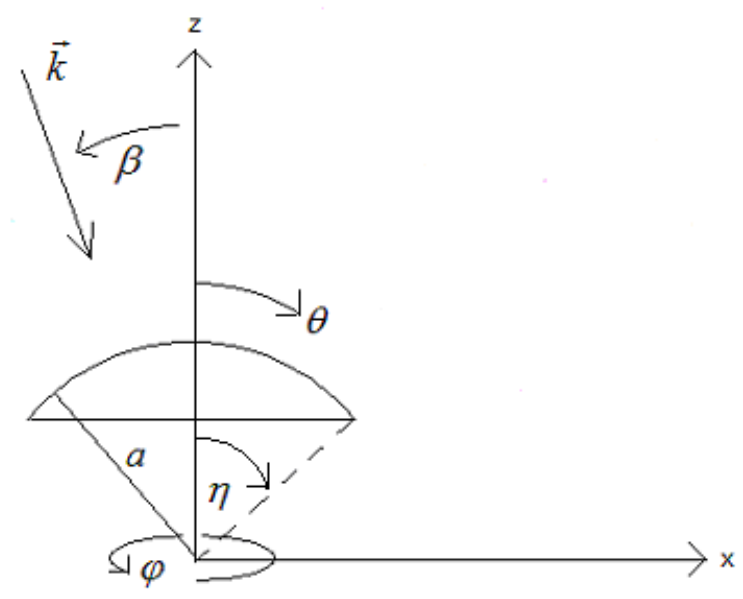

Figure 8 - Geometry of the spherical cap.

The relevant parameters of the Kirchhoff integral are given by 


$$
\begin{gathered}
\vec{k}=-k \sin \beta \hat{x}+k \cos \beta \hat{z} \\
\hat{n}=\cos \varphi \sin \theta \hat{x}+\sin \varphi \sin \theta \hat{y}+\cos \theta \hat{z} \\
\vec{r}^{\prime}=a \cos \varphi \sin \theta \hat{x}+a \sin \varphi \sin \theta \hat{y}+a \cos \theta \hat{z} \\
d \sigma=a^{2} \sin \theta d \theta d \varphi .
\end{gathered}
$$

Substituting into the Kirchhoff integral and simplifying,

$$
f=\frac{i k a^{2}}{2 \pi} \int_{0}^{2 \pi} \int_{0}^{\eta}(\sin \beta \cos \varphi \sin \theta-\cos \beta \cos \theta) \sin \theta e^{i 2 k a(\sin \beta \cos \varphi \sin \theta-\cos \beta \cos \theta)} d \theta d \varphi
$$

This author is not aware of an analytic solution, so this double integral is to be evaluated numerically.

\subsection{Solving the Kirchhoff Integral Numerically}

Using a simplified approximation to the geometry of a complex shape in order to obtain a closed form, analytic solution to the Kirchhoff integral can introduce errors into the predicted scattering associated to the geometric differences. In cases where analytic simplification is impractical, or the resulting errors are unacceptable, it is possible to use a numerical approach to solve the Kirchhoff integral that does not rely on simplifying the object's shape. In this section, a method is presented for solving the Kirchhoff integral numerically, regardless of the object's irregularities. 


\subsubsection{Creating Geometry and a Mesh in COMSOL Multiphysics}

The first step in solving the Kirchhoff integral numerically is to create a surface mesh of the object of interest. This is simply a collection of small connected triangles that attempt to match the surface of the object. The size of these triangles determines how well they conform to the shape. One simple method for creating the object's surface mesh is through use of the finite element software COMSOL Multiphysics ${ }^{\circledR}$ (ver. 3.2a), referred to in this work simply as COMSOL. COMSOL is a commercially available program for solving finite element method problems, which will be explored further in Chapter 3. For now, it is used for its capability to model and mesh complex geometries.

Within COMSOL, the geometry of the object of interest can be created using either the GUI or script. Objects can be created from scratch or imported as computer-aided design (CAD) files. Once the geometry is created, a mesh can be generated to conform to it. The mesh for a three dimensional object consists of tetrahedrons in the interior and triangles on the surfaces. The vertices of these shapes are known as mesh vertices. The mesh is created by defining input parameters that regulate the size of the triangles and tetrahedrons and how well they conform to curved parts of the geometry. The size of the triangles on the geometry's surface will have an effect on the accuracy of numerical solution to the integral, since each triangle acts a differential element.

The mesh may be represented by two sets of information: coordinates and connectivity. The coordinate information lists the location of each of the numbered mesh vertices. The connectivity lists the vertices (by number) that make up each of the mesh elements, in some systematic way (e.g. counterclockwise for triangles, right hand rule for tetrahedrons). In this way, each mesh tetrahedron is defined by a connectivity of four vertices whose locations are 
contained in the coordinate list. Similarly, each triangle is defined by three vertices. Because the Kirchhoff method uses a surface integral, only the information regarding the surface triangles is important. This can easily be separated and assembled within COMSOL into two arrays.

\subsubsection{Determination of Insonified Surface}

Once the two surface mesh arrays have been compiled, the next step is to determine which triangular surface elements should contribute to the solution. Because the Kirchhoff integral is only applied to the insonified region of the object, any surface in the shadow needs to be ignored. When solving the integral analytically, the proper surface of the object is defined by the limits of integration. Since these are not defined here, there needs to be some way to eliminate the triangle elements that are not in the line of sight of the incoming wave vector. This problem is similar to one in computer graphics, where several surfaces or polygons share the same set of pixels. In order to display the correct surface (e.g. that which is closest to the viewer), a process known as hidden surface removal determines what information each pixel should display (De Berg et al., 1997). Newell's algorithm is a similar method for determining which polygons are hidden (Newell, 1972). A simplified version of Newell's algorithm is

developed here in order to determine which triangles are hidden by others, from the view-point of the incoming wave vector.

To use the method developed here, a step must be taken before the mesh is created. In order to avoid complicated coordinate transforms later on, the incoming wave vector is set to always coincide with the negative $\mathrm{z}$ direction. The object can be rotated in COMSOL so that the desired angle of incidence is achieved. After the mesh is created for this orientation of the object, the simplified version of Newell's algorithm can be applied: 
1) Sort elements by max $z$ value

2) Minmax test in $x$

3) Minmax test in $y$

4) Rasterization test

For the first step, the elements can be sorted by their maximum $\mathrm{z}$ values by comparing the positions of each triangle's three vertices. Because the incoming wave vector travels in the negative $\mathrm{z}$ direction, the elements higher on this list are more likely to be part of the front surface.

The remainder of the algorithm involves determining whether elements are covered by others. If the projections into the $x-y$ plane of two elements intersect, then the element with the greater $\mathrm{z}$ value covers the other. Elements which are covered by others must lie on a surface that is not in the line of sight of the incoming wave vector and therefore in the shadow. These hidden elements are removed from the list as they are found. The tests proceed by starting with the first element in the list and comparing it to all the other elements below it in the list. When these comparisons are finished and covered elements are removed, the second element in the list is compared to all the other elements below it. This process eliminates redundant comparisons.

The first test is a minmax test in $\mathrm{x}$ : determining whether the two elements share any $\mathrm{x}$ values by comparing minimum and maximum values. If this test fails (they do not share common $x$ values), the second element is not covered by the first, and the next element is compared. 


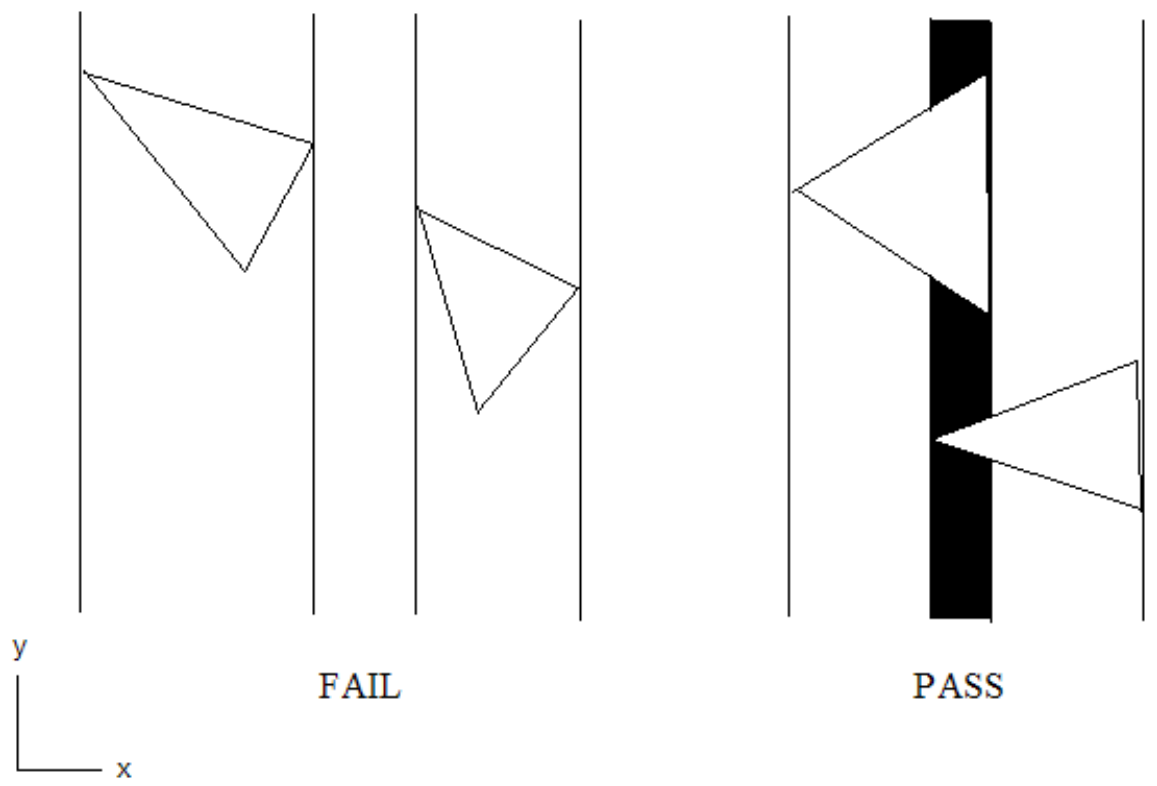

Figure 9 - Examples of minmax tests in $\mathrm{x}$ for Newell's algorithm.

If the two elements pass the minmax tests in $\mathrm{x}$ and $\mathrm{y}$ and share values in both $\mathrm{x}$ and $\mathrm{y}, \mathrm{a}$ rasterization test is performed. Given a sampling rate, a rectangle of $x-y$ points is created; the coordinates of these points are determined by the minimum and maximum $\mathrm{x}$ and $\mathrm{y}$ values of the first element. Each point is sampled to determine if it is part of either triangular element. Points on the border between two triangles are considered part of neither. If a point is part of both, then the first element must cover the second, which is then removed from the list. This test is run only after a set of elements has passed both minmax tests to save run time.

\subsubsection{Evaluating the Kirchhoff Integral Numerically}

With the final list of elements representing the object's front surface, the Kirchhoff integral can now be evaluated. There is some ambiguity in the direction of the normal vectors that must be clarified. Due to the numbering scheme in the COMSOL connectivity list, 
determining the cross product of the triangle edges does not always provide an outward normal. This can be fixed by noting that the dot product of the normal and unit wave vectors should either be negative or zero, because the faces are either oriented toward the positive $\mathrm{z}$ direction or are perpendicular to it. None of the triangles can be facing the negative $\mathrm{z}$ direction after applying Newell's algorithm. For the elements perpendicular to the wave vector, it is not important if the normal vector faces inward or outward since the dot product and integrand reduces to zero in either case. For the other faces however, it is simply enough to compute the absolute value of the dot product of the normal and unit wave vectors and set it negative. For

the $\vec{k}_{i n c} \cdot \vec{r}^{\prime}$ term in the exponent, the average of the dot products of the wave vector and position vectors of each vertex is used. The area of each triangle is computed for the differential element term. Finally, the expression is summed over each triangle element and the Kirchhoff integral can be numerically evaluated.

\subsection{Comparison of Methods for Solving the Kirchhoff Integral}

The numerical method of solving the Kirchhoff integral can be tested against some of the analytic solutions derived earlier. In each case, the mesh is made fine enough so that there is good agreement between the two methods. The mesh size at which the numerical solution converged to the analytic solution varied in each case, and proved highly dependent on the geometry of the scatterer. Therefore, a simple rule for determining an appropriate mesh size was not ascertained. 


\subsubsection{Sphere}

A sphere of radius $5 \mathrm{~cm}$ was created in COMSOL and meshed, seen in figure 10. Because of its symmetry, no care needed to be taken to orient the sphere in any fashion.

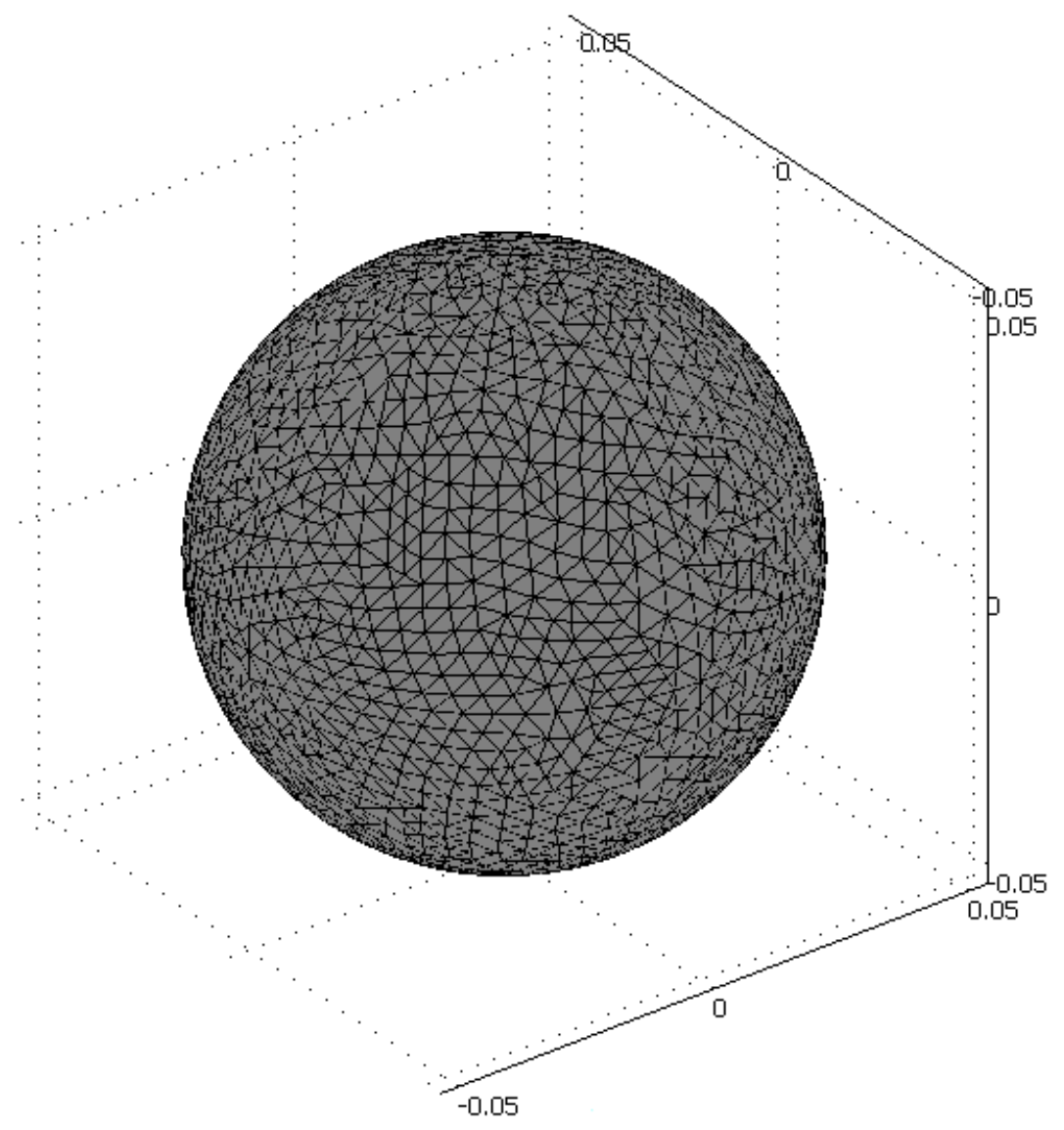

Figure 10 - Surface mesh of a sphere of radius $5 \mathrm{~cm}$ in COMSOL.

This mesh had a maximum element size of $0.5 \mathrm{~cm}$, giving 4266 surface triangle elements.

Extracting the connectivity and node coordinate information, these elements were subjected to the Newell's algorithm in the technical computing software MATLAB® (ver. 7.2.0.232). To visualize the effect of this, the remaining triangles are seen plotted in figure 11. 


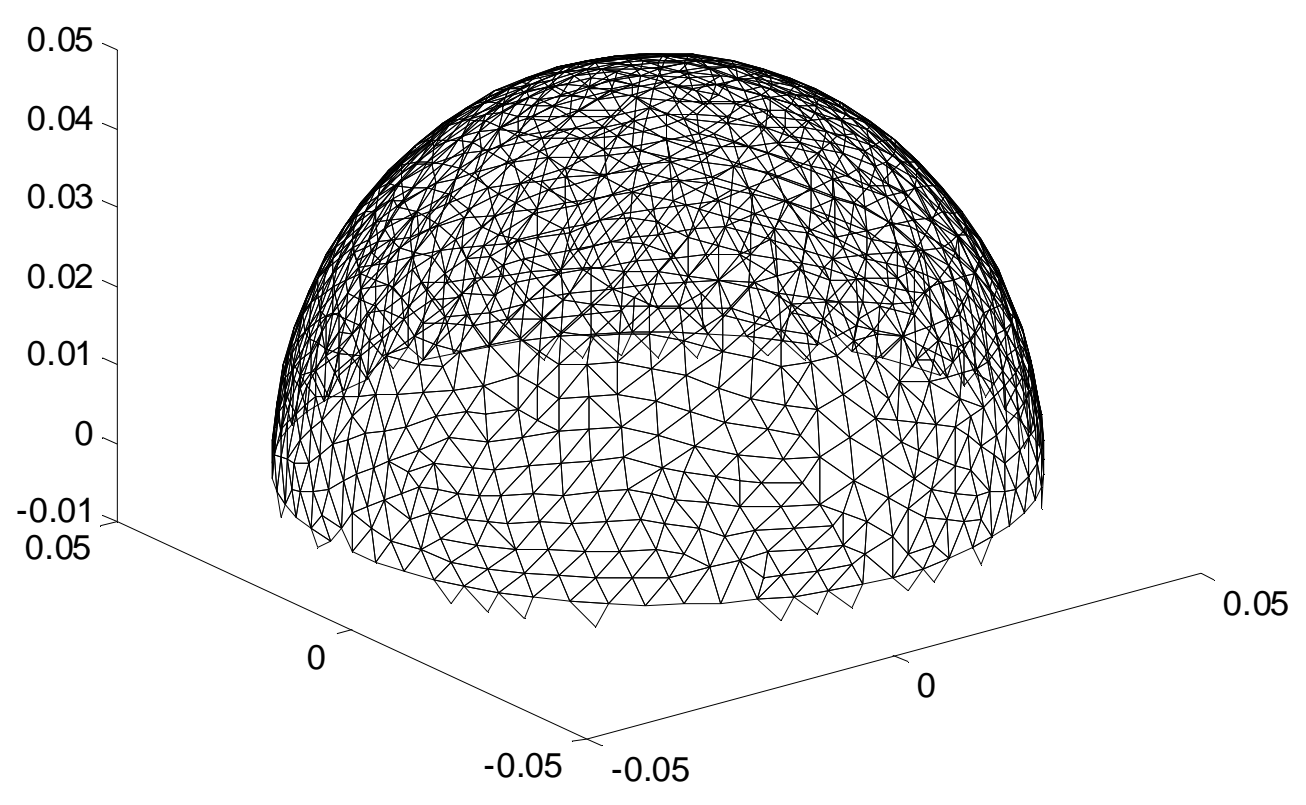

Figure 11 - Surface mesh of a sphere in MATLAB after Newell's algorithm.

Newell's algorithm has the desired effect of removing the elements out of the line of site of the incoming wave vector from above. To ensure that each of the triangles in the plot were represented by an element and not simply formed by surrounding triangles, the midpoint of each were plotted, seen in figure 12. 


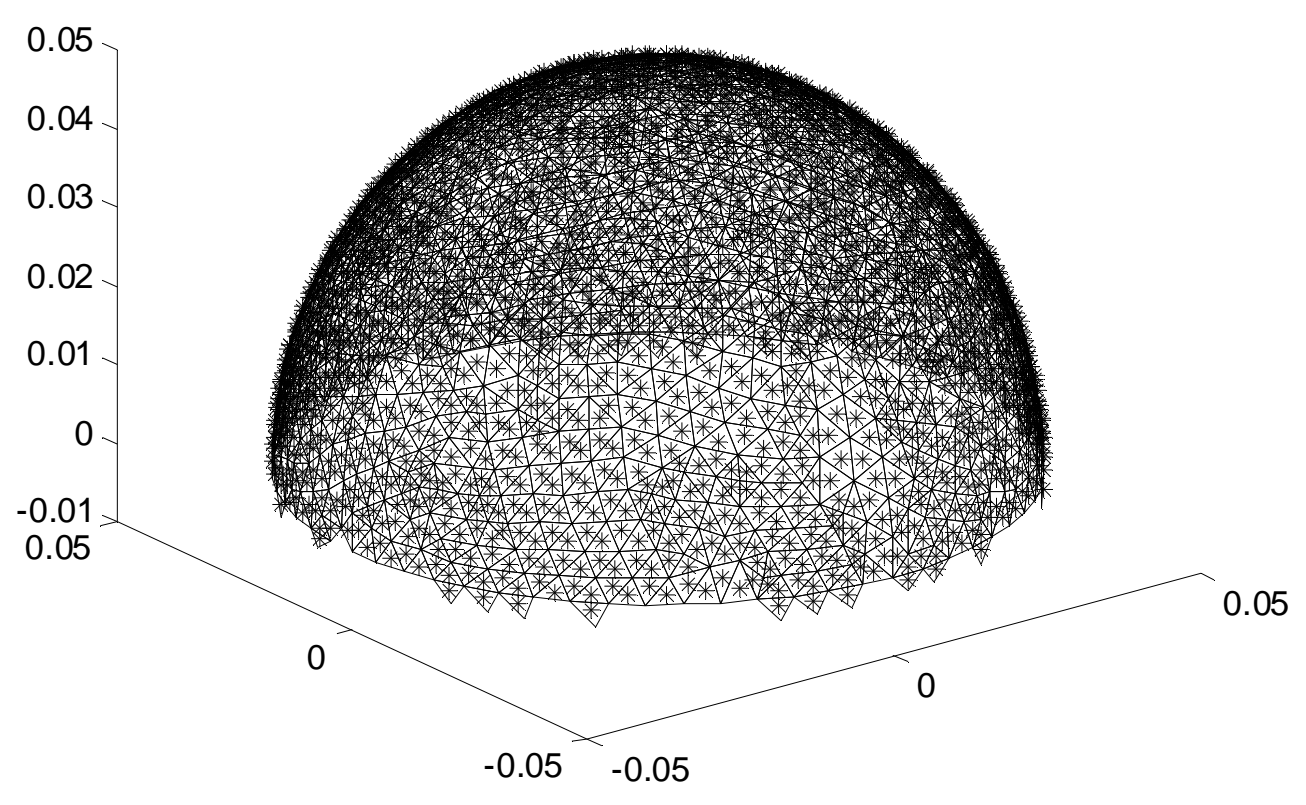

Figure 12 - Surface mesh of a sphere in MATLAB after Newell's algorithm, with triangle midpoints.

Taking a closer look, it is clear that Newell's algorithm kept all the desired triangular elements.

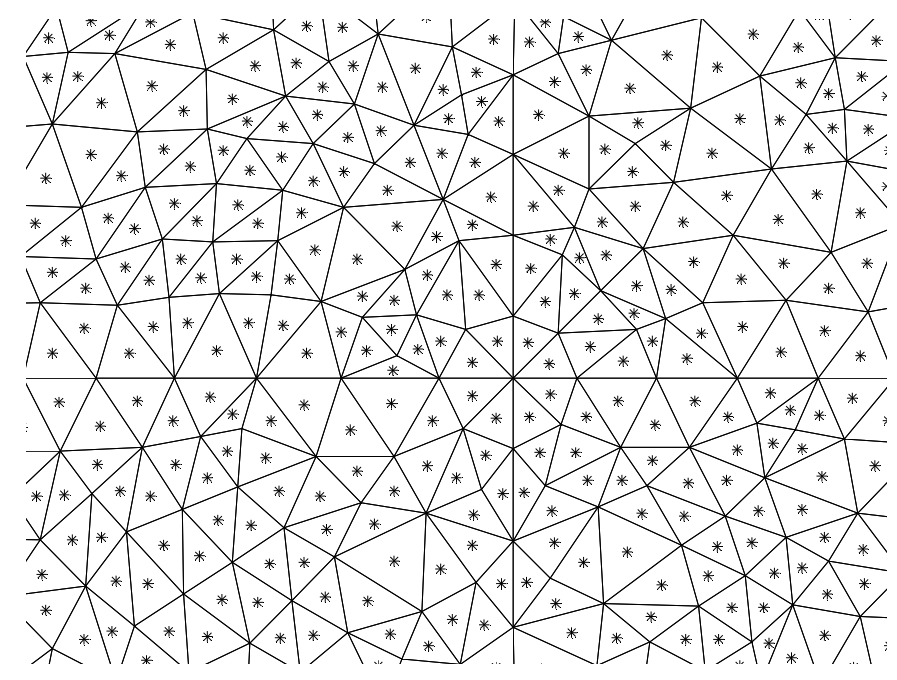

Figure 13 - Close view of surface mesh of sphere, with midpoints. 
Finally, the Kirchhoff integral was solved numerically over each element and compared to the analytic solution for a rigid/fixed sphere (section 2.3.1) for different values of ka, the product of the wavenumber and the sphere's radius, shown in figure 14.

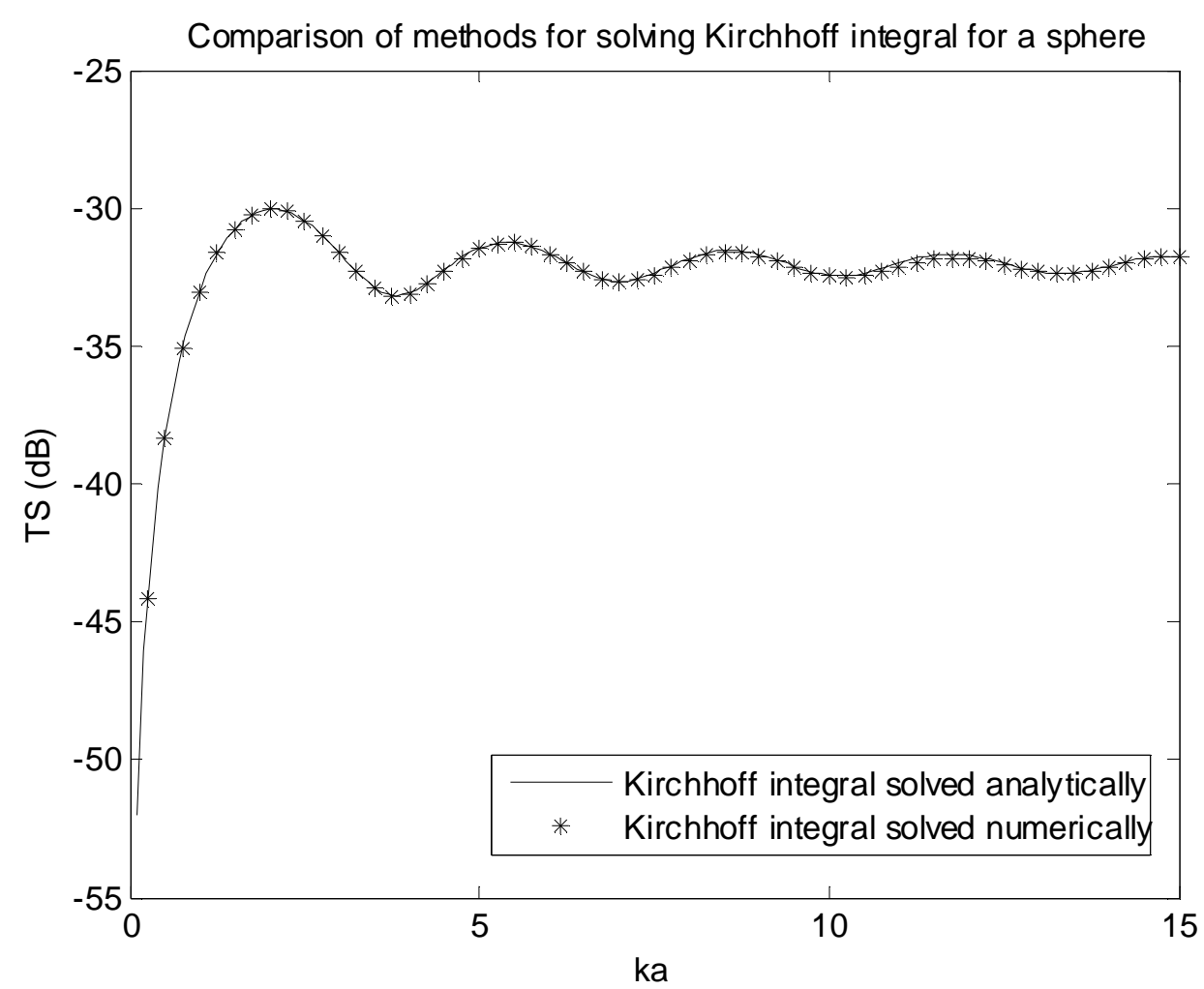

Figure 14 - Target strength of a rigid/fixed sphere with radius $5 \mathrm{~cm}$ by solving the Kirchhoff integral analytically (solid line) and numerically (stars).

By using a sufficiently fine surface mesh, the numerical solution converged to the analytic solution. 


\subsubsection{Finite Cylinder}

A finite cylinder of length $10 \mathrm{~cm}$ and radius $1 \mathrm{~cm}$ centered at the origin was created in COMSOL, oriented with its lengthwise axis along the $\mathrm{x}$ axis so that the incoming wave vector, along the negative $\mathrm{z}$ axis, met it at broadside. The cylinder was then meshed with a maximum element size of $0.15 \mathrm{~cm}$, producing 11170 surface elements.

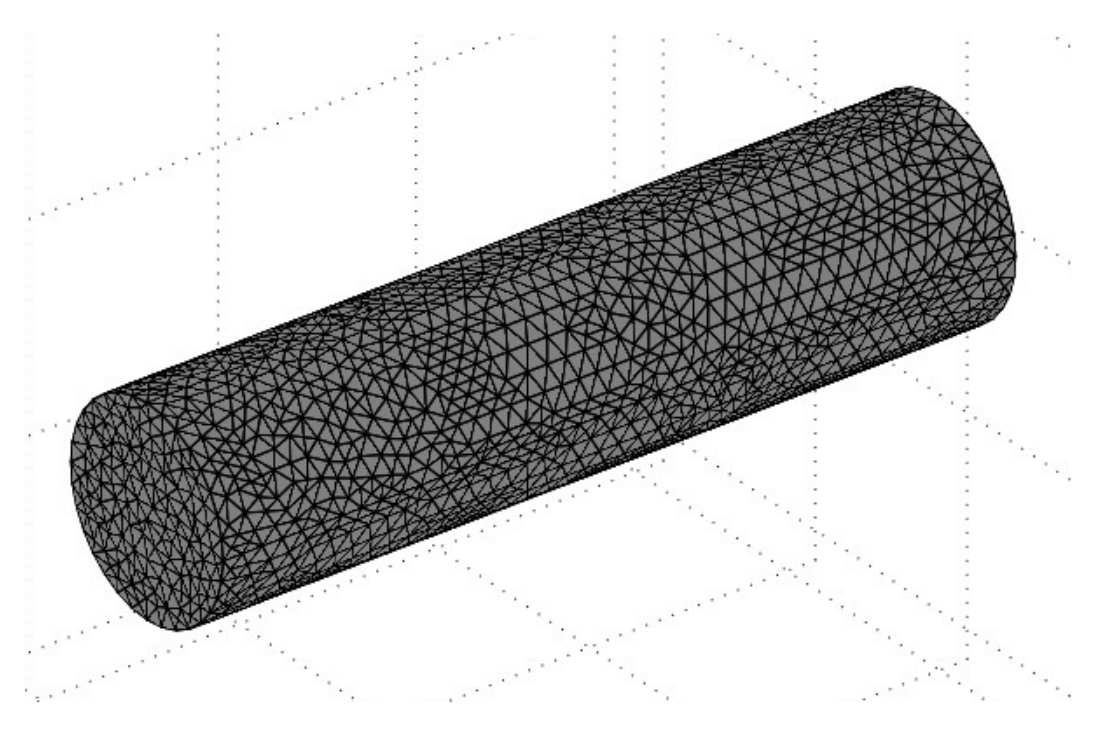

Figure 15 - Surface mesh of a finite cylinder in COMSOL.

Again, the triangular elements were run through Newell's algorithm in MATLAB, with the remaining ones shown in figure 16. 


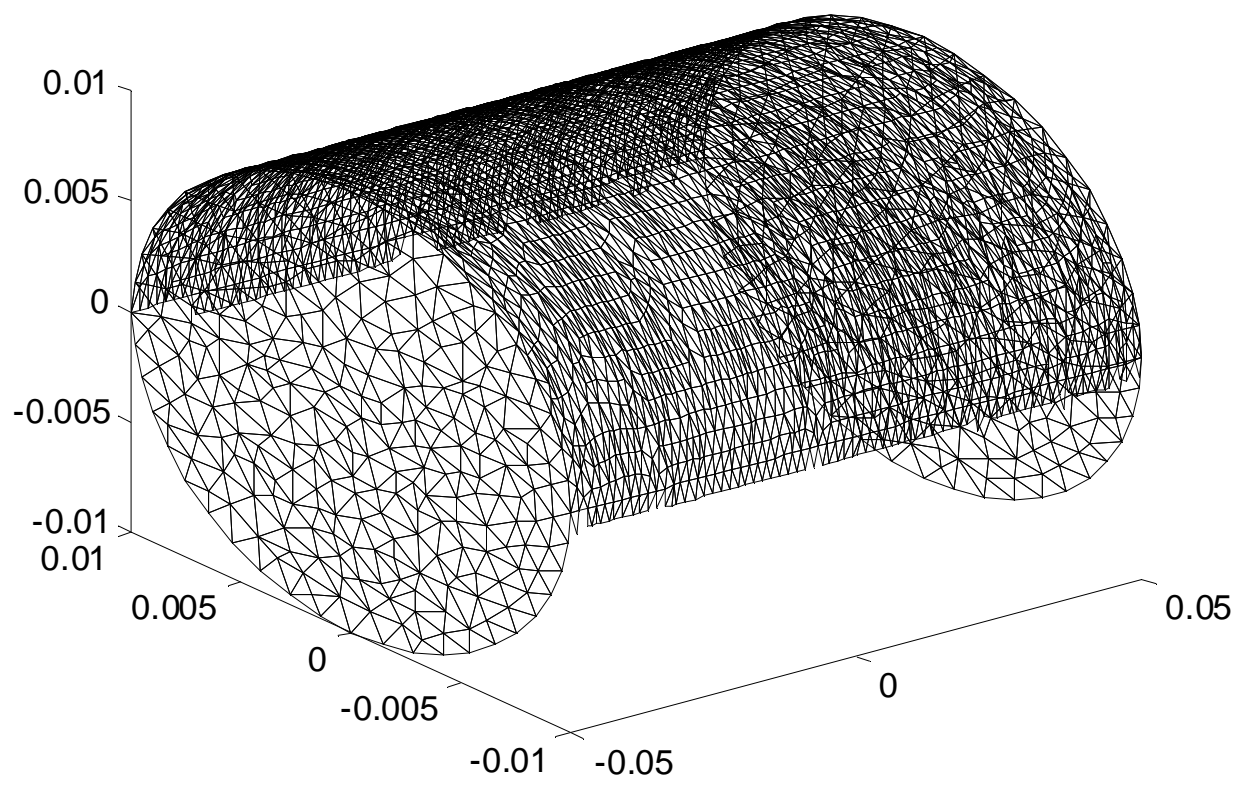

Figure 16 - Surface mesh of a finite cylinder in MATLAB after Newell's algorithm.

Note that the elements on the ends of the cylinder were not removed. This is due to a shortcoming in the rasterization test in Newell's algorithm. Because the elements are vertical, they cannot be sampled in the $x-y$ plane. However, because the dot product of the unit normal and unit wave vector is zero, their contribution amounts to zero regardless. The Kirchhoff integral can now be evaluated over all the remaining elements and compared to the analytic solution for a finite cylinder (section 2.4.1) over a range of values of ka, the product of the wavenumber and the cylinder's radius. Again, the numerical results converge to the analytic solution, as seen in figure 17. 


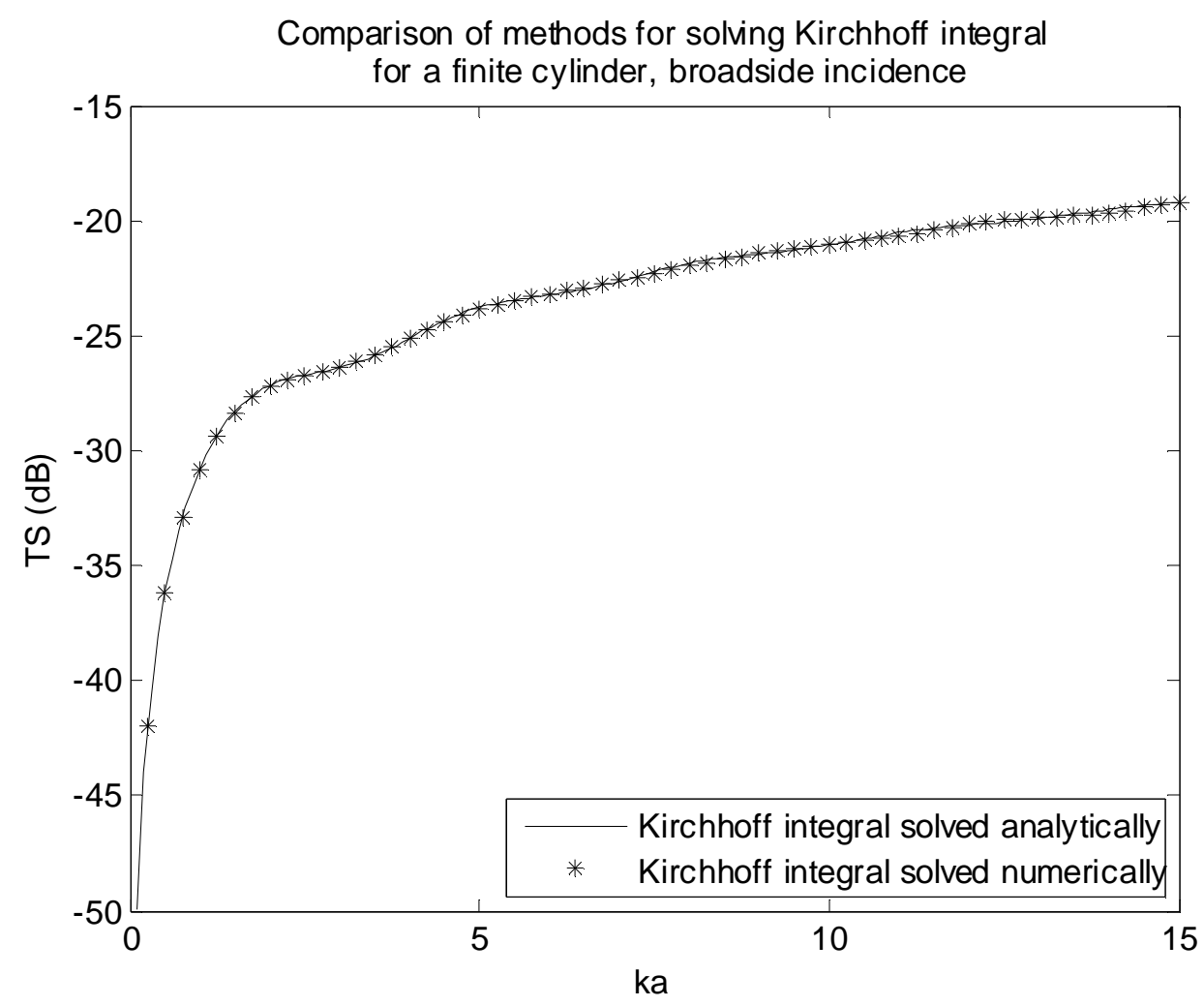

Figure 17 - Target strength of a rigid/fixed finite cylinder with length $10 \mathrm{~cm}$ and radius $1 \mathrm{~cm}$ by solving the Kirchhoff integral analytically (solid line) and numerically (stars).

\subsubsection{Disk}

A disk of radius $5 \mathrm{~cm}$ and thickness $1 \mathrm{~cm}$, centered at the origin, was created in COMSOL, oriented so that the incoming wave vector along the negative $\mathrm{z}$ axis arrived normal to the round face. The surface mesh had a maximum element size $0.25 \mathrm{~cm}$, giving 10640 surface triangles. 


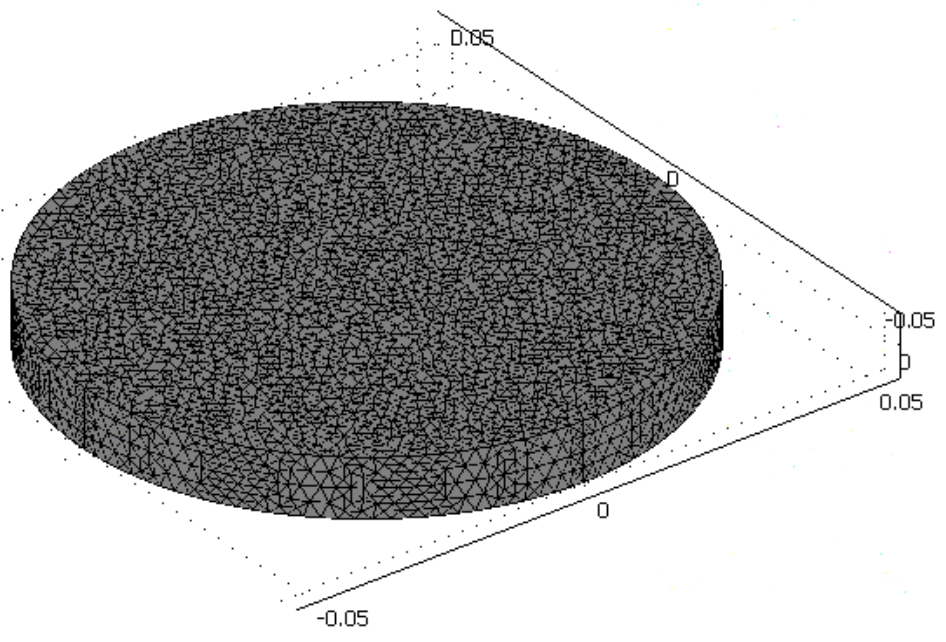

Figure 18 - Surface mesh of a disk in COMSOL.

The triangular elements were then run through Newell's algorithm in MATLAB, with the results shown in figure 19.

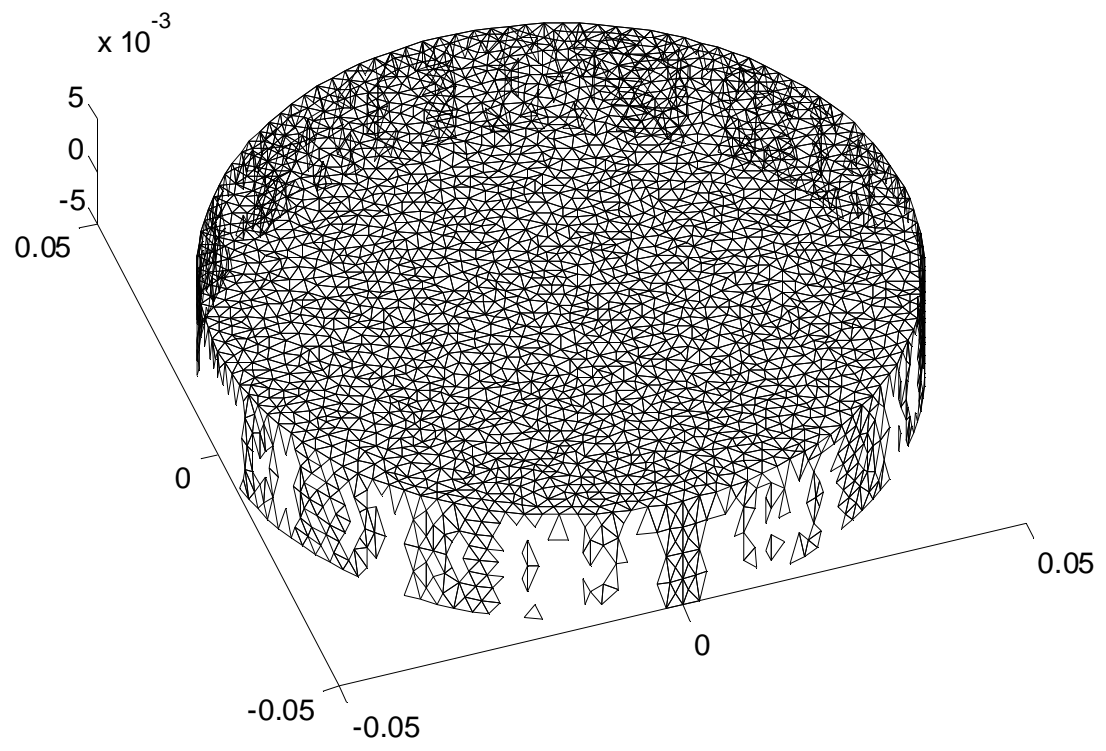

Figure 19 - Surface mesh of a disk in MATLAB after Newell's algorithm. 
Many of the vertical elements on the side of the disk remain, as they did on the ends of the finite cylinder; however, they do contribute to the solution. Finally, the Kirchhoff integral can be solved numerically over all the remaining elements, and the solution is compared to the analytic solution (section 2.5.2). Again the numerical results matched the analytic solution, as seen in figure 20 .

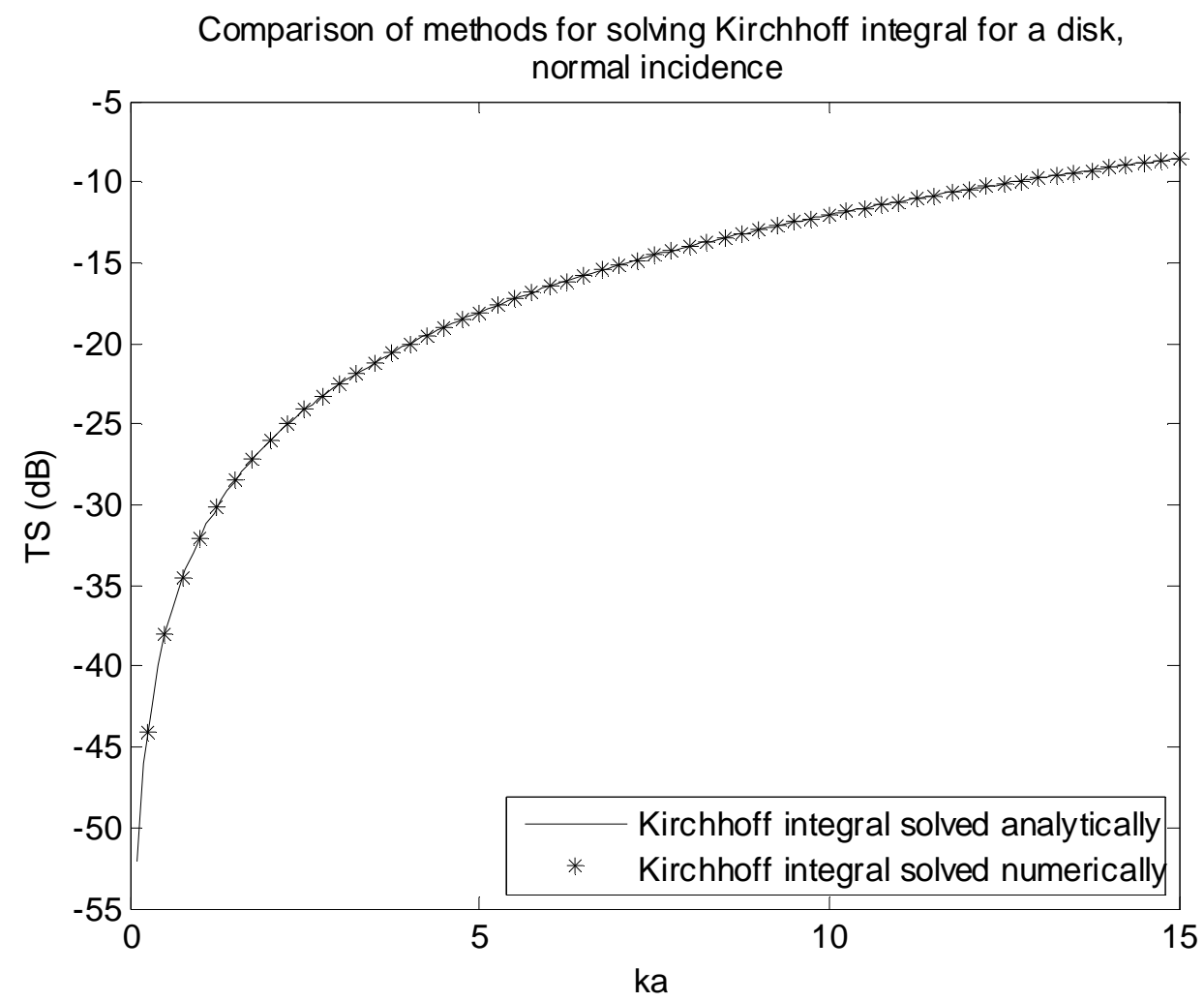

Figure 20 - Target strength of a rigid/fixed disk with radius $5 \mathrm{~cm}$ and thickness $1 \mathrm{~cm}$ by solving the Kirchhoff integral analytically (solid line) and numerically (stars). 


\subsubsection{Spherical Cap}

To generate the spherical cap, a sphere of radius $10 \mathrm{~cm}$ was first created in COMSOL, centered at the origin. The top 30 degrees of the sphere were kept, leaving a cap of radius $5 \mathrm{~cm}$ and thickness $1.34 \mathrm{~cm}$. The cap was oriented so that the incoming wave along the negative $\mathrm{z}$ axis arrived normal to its top. It was meshed with a maximum element size of $0.25 \mathrm{~cm}$, giving 9382 surface elements.

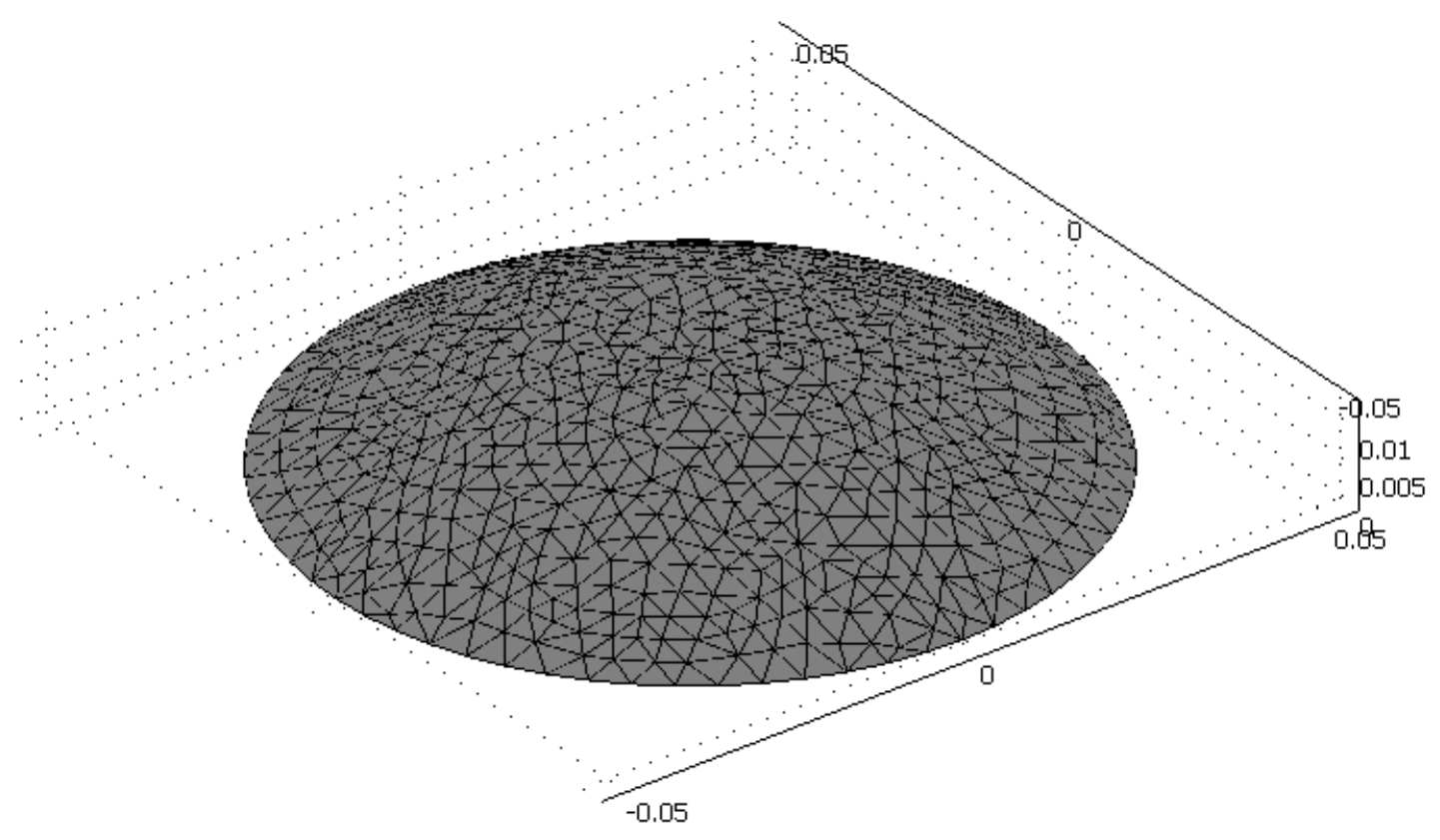

Figure 21 - Surface mesh of a spherical cap in COMSOL.

The triangular elements were then run through Newell's algorithm in MATLAB, with the results plotted in figure 22. 


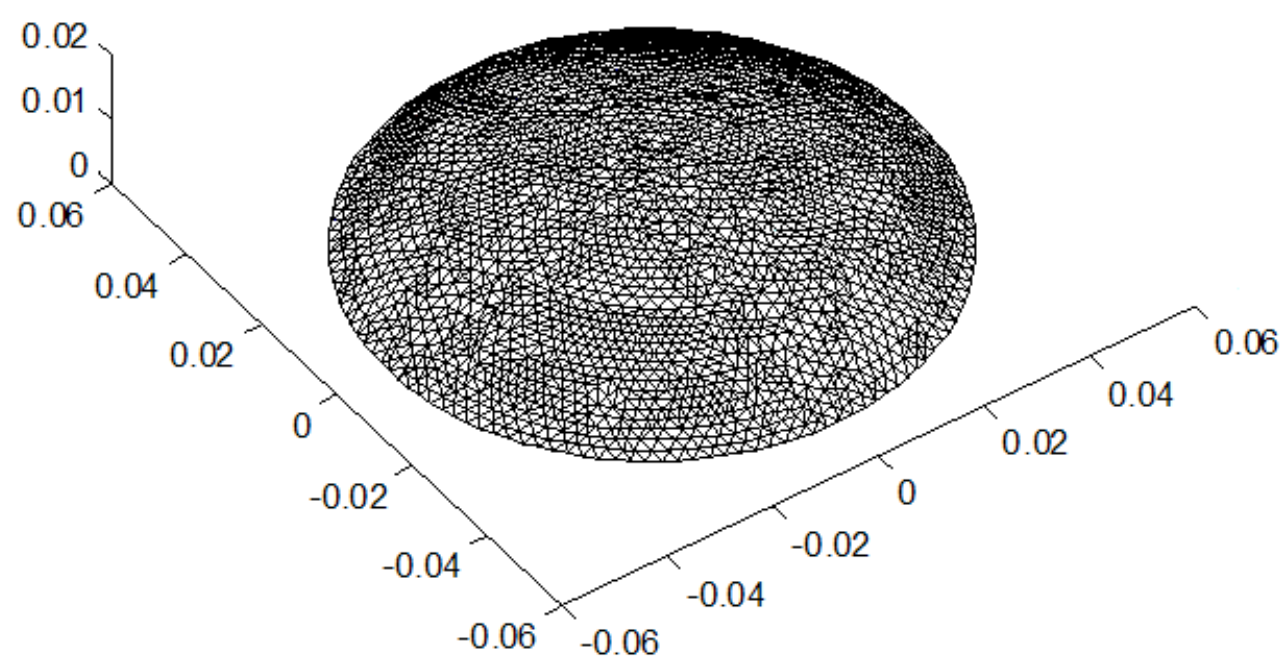

Figure 22 - Surface mesh of a spherical cap in MATLAB after Newell's algorithm.

Finally, the Kirchhoff integral was solved numerically over all the remaining elements and compared to the analytic solution for a spherical cap (section 2.5.3) over a range of ka, where a is the radius of the cap. Again, the numerical results match the analytic solution, as seen in figure 23 . 


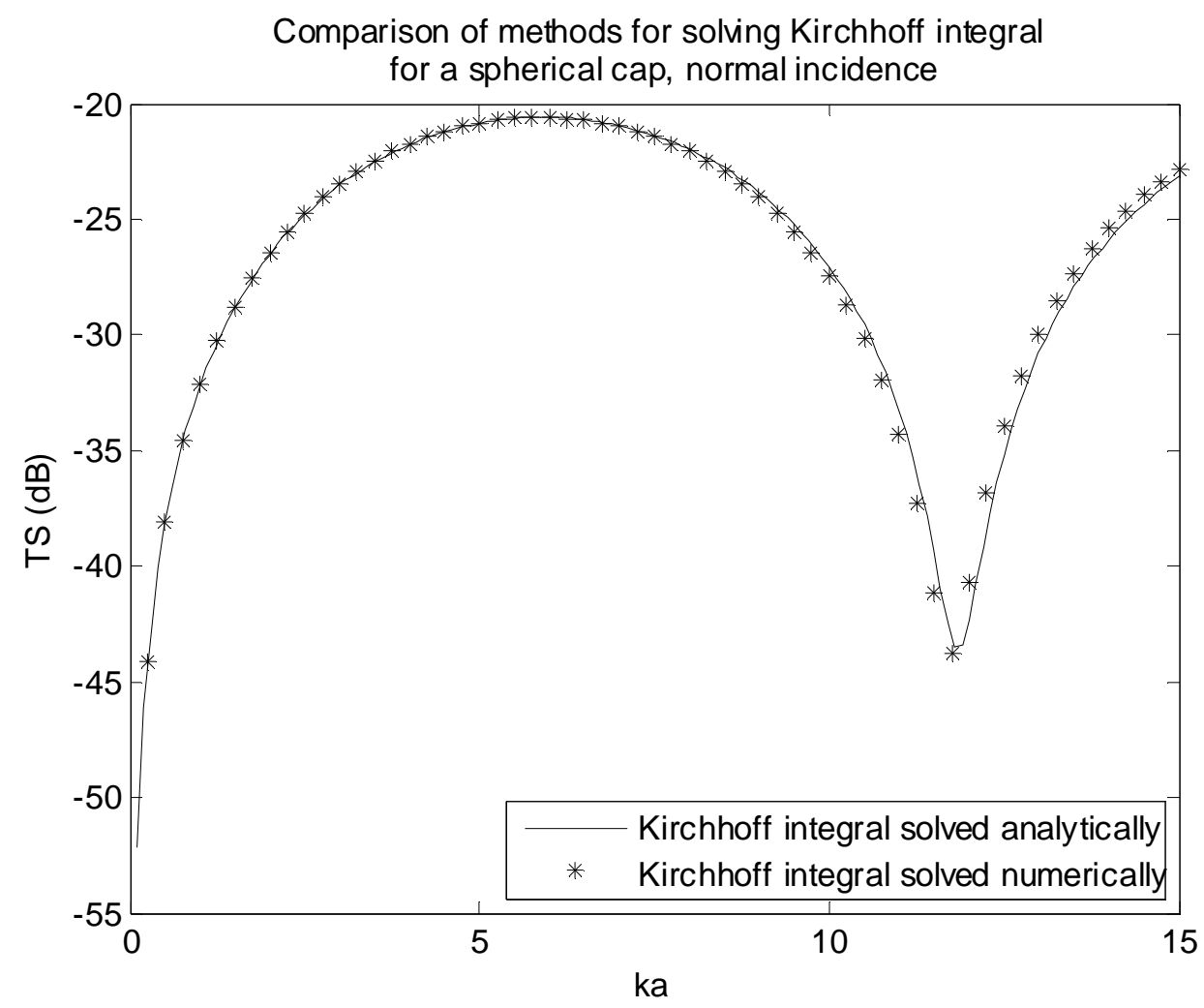

Figure 23 - Target strength of a rigid/fixed spherical cap with radius $5 \mathrm{~cm}$ and radius $1.34 \mathrm{~cm}$ by solving the Kirchhoff integral analytically (solid line) and numerically (stars).

\subsection{Chapter Summary}

In this chapter, the Kirchhoff method has been explored for understanding scattering from objects with and without known exact solutions. The derived solutions from this method have their limitations and may not be valid in many situations, depending on factors such as boundary condition and incident wavelength. However, as will be seen, this method proves to be useful in studying the scattering from sand dollars at certain frequencies and angles of orientation. The analytic solutions for a disk and spherical cap are compared to experimental scattering data from a sand dollar in Chapter 4. The numerical approach to solving the Kirchhoff integral was presented in cases where representing a complex geometry with a 
simpler one introduces too many errors. This approach has been shown to agree very well with analytic solutions to the Kirchhoff integral. Also in Chapter 4, the scattering from a rigid/fixed three dimensional model of the sand dollar is calculated with this numerical method and compared to experimental results. 


\section{Chapter 3 The Finite Element Method}

\subsection{Introduction}

In this chapter, the theory behind the finite element method and its use in acoustic scattering problems are explained. This is presented in the form of a general problem of acoustic scattering from a rigid/fixed object. A technique for reducing random numerical error in the solution of two and three dimensional acoustic scattering problems is then presented. COMSOL Multiphysics, a program for solving finite element method problems is explored. A particular focus concerns the radiation condition used by this program. The finite element method is tested for scattering from a rigid/fixed infinite cylinder and a rigid/fixed sphere, problems with known exact solutions. The methodology for modeling the sand dollar using the finite element method is then discussed.

\subsection{Theoretical Background}

The finite element method is a general numerical technique for solving boundary value problems. Its application to acoustic scattering problems is now shown by introducing a general three dimensional problem. Consider a harmonic plane wave $p_{i n c}=p_{0} e^{i \vec{k} \cdot \vec{x}}$ that is incident upon a rigid/fixed object in free space, represented by $\bar{\Omega}$. The surface of the object is labeled $\Gamma$. 


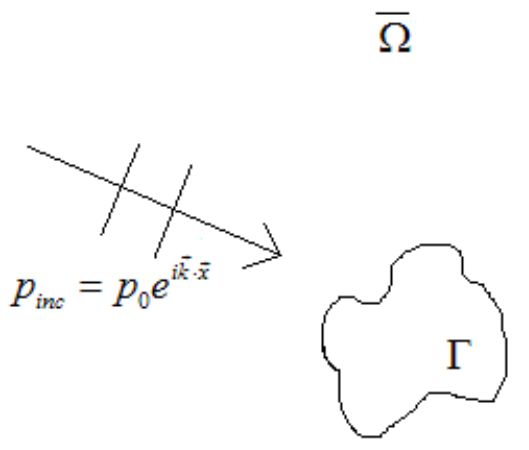

Figure 24 - Domain of three dimensional scattering problem, shown with incident wave.

The governing equation for the total pressure field in the domain $\bar{\Omega}$ from the object is the Helmholtz equation, the time independent form of the wave equation,

$$
\nabla^{2} p+k^{2} p=0 \text { in } \bar{\Omega} .
$$

The total pressure is the sum of the incident and scattered pressure fields,

$$
p=p_{\text {inc }}+p_{\text {scat }} .
$$

Therefore, the scattered pressure field, $p_{\text {scat }}$, also satisfies the Helmholtz equation in $\bar{\Omega}$,

$$
\nabla^{2} p_{\text {scat }}+k^{2} p_{\text {scat }}=0 \text { in } \bar{\Omega} \text {. }
$$

The goal of the problem is to determine the scattered pressure field.

On the boundary of the object, $\Gamma$, the rigid/fixed condition requires that the normal gradient of total pressure is zero,

$$
\vec{n} \cdot \nabla p=0,
$$

where $\vec{n}$ is the surface normal of the object. This is easily written for the scattered pressure in terms of the incident pressure,

$$
\vec{n} \cdot \nabla p_{\text {scat }}=-\vec{n} \cdot \nabla p_{i n c} .
$$


To complete the boundary value problems, a condition of the behavior of the scattered pressure at infinity is required, given by the Sommerfeld radiation condition (Sommerfeld, 1949) in three dimensions as

$$
\lim _{r \rightarrow \infty}\left[r\left(\frac{\partial p_{\text {scat }}}{d r}-i k p_{\text {scat }}\right)\right]=0
$$

This condition describes the behavior of the scattered pressure as $r$, the distance from the scatterer, goes to infinity.

Note that the problem above is defined on the unbounded domain $\bar{\Omega}$. The finite element method used in this thesis requires a domain where the boundary value problem is defined to be bounded. Therefore, the Sommerfeld condition is substituted with an approximate radiation condition defined on the boundary $\Gamma_{\text {inf }}$ that contains the object. The geometry of this new bounded region, labeled $\Omega$, is seen in figure 25 .

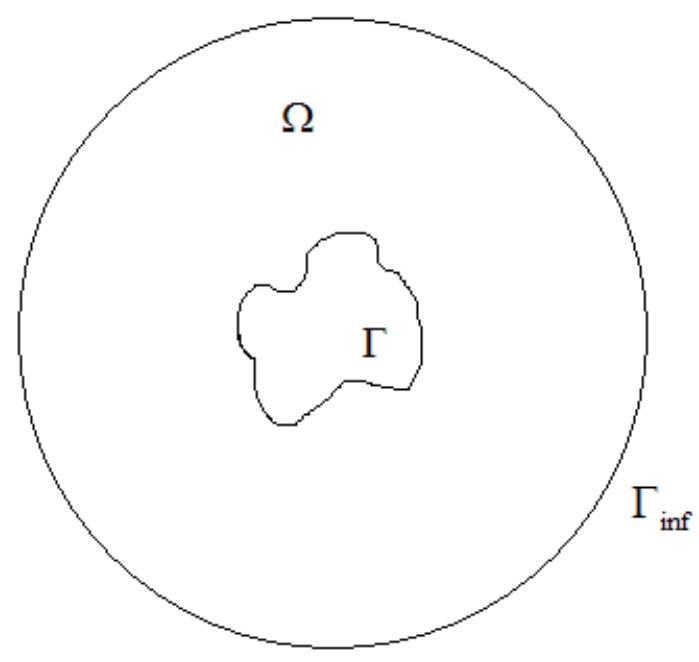

Figure 25 - Geometry of a bounded three dimensional scattering problem.

At $\Gamma_{\text {inf }}$, the condition used instead of equation (3.6) is defined as 


$$
\frac{\partial p_{s c a t}}{\partial r}=i k p_{s c a t} \text { on } \Gamma_{\text {inf }}
$$

and equation (3.3) is defined over $\Omega$. Therefore, the boundary value problem that will be solved with the finite element method here is summarized as

$$
\begin{gathered}
\nabla^{2} p_{\text {scat }}+k^{2} p_{\text {scat }}=0 \text { in } \Omega, \\
\vec{n} \cdot \nabla p_{\text {scat }}=-\vec{n} \cdot \nabla p_{\text {inc }} \text { on } \Gamma, \\
\frac{\partial p_{\text {scat }}}{\partial r}=i k p_{\text {scat }} \text { on } \Gamma_{\mathrm{inf}} .
\end{gathered}
$$

To apply the finite element method, the boundary value problem, given in (3.8), is written in the form of a variation equation, as shown in the following development. First the condition on $\Omega$ is multiplied by a weight function $w \in V$ and integrated over the domain, resulting in

$$
\int_{\Omega} w^{*}\left[\nabla^{2} p_{\text {scat }}+k^{2} p_{\text {scat }}\right] d \Omega=0 \quad \forall w \in V
$$

$V$ is a linear space of functions which will be defined in a moment, and the asterisk denotes the operation of conjugation. The first term on the left side on equation (3.9) is integrated by parts, using the identity

$$
\operatorname{div}(f \nabla g)=\nabla f \cdot \nabla g+f \nabla^{2} g .
$$

This identity is valid for any two scalar fields $f$ and $g$ which are twice differentiable.

Integrating this identity over the domain $\Omega$ and using the divergence theorem (Strang, 1991) gives

$$
\int_{\Omega} f \nabla^{2} g d \Omega=-\int_{\Omega} \nabla f \cdot \nabla g d \Omega+\int_{\partial \Omega} f \nabla g \cdot \vec{n} d \Omega,
$$


where $\vec{n}$ is the unit normal vector outward from the boundary of $\Omega$. Note that the right side of this expression requires $f$ and $g$ to be only first-order differentiable. Using equation (3.11) in equation (3.9) with $f$ substituted with $w^{*}$ and $g$ by $p_{\text {scat }}$ gives

$$
\int_{\Omega}\left(-\nabla w^{*} \cdot \nabla p_{\text {scat }}+k^{2} w^{*} p_{\text {scat }}\right) d \Omega+\int_{\partial \Omega} w^{*} \nabla p_{\text {scat }} \cdot \vec{n} d \Gamma=0 \quad \forall w \in V
$$

Using $\partial \Omega=\Gamma+\Gamma_{\text {inf }}$ and the boundary conditions in (3.8) gives the following variational equation for the scattered field $p_{\text {scat }} \in V$

$$
\int_{\Omega}\left(\nabla w^{*} \cdot \nabla p_{\text {scat }}-k^{2} w^{*} p_{\text {scat }}\right) d \Omega-\int_{\Gamma_{\mathrm{inf}}} i k w^{*} p_{\text {scat }} d \Gamma_{\mathrm{inf}}=-\int_{\Gamma} w^{*} \nabla p_{\text {inc }} \cdot \vec{n} d \Gamma
$$

$$
\forall w \in V
$$

From this equation, the conditions on the space $V$ are deduced: the functions have to be squareintegrable with square-integrable gradients. Equation (3.13) can be written in compact form by introducing the notation

$$
\begin{gathered}
a\left(w, p_{\text {scat }}\right)=\int_{\Omega}\left(\nabla w^{*} \cdot \nabla p_{\text {scat }}-k^{2} w^{*} p_{\text {scat }}\right) d \Omega-\int_{\Gamma_{\mathrm{inf}}} i k w^{*} p_{\text {scat }} d \Gamma_{\mathrm{inf}} \\
\gamma(w)=-\int_{\Gamma} w^{*} \nabla p_{\text {inc }} \cdot \vec{n} d \Gamma
\end{gathered}
$$

so that the variational equation is

$$
a\left(w, p_{\text {scat }}\right)=\gamma(w) \quad \forall w \in V
$$

Note that the form $a(\cdot, \cdot)$ is linear with respect to the first and second arguments and that $\gamma(\cdot)$ is linear with respect to its argument as well.

The finite element method is a numerical technique to construct approximate solutions to variational problems such as (3.16). An approximation to the infinite-dimensional space $V$ is 
constructed. This space is denoted $V^{h}$ and has the property that it is a subspace of $V$. The problem is solved in this new space, that is, the goal is to determine $p^{h}$ such that

$$
a\left(w^{h}, p^{h}\right)=\gamma\left(w^{h}\right) \quad \forall w^{h} \in V^{h}
$$

The space $V^{h}$ is a finite dimensional space, which means that a basis can be constructed using a finite number of terms. The elements of that basis are denoted $\phi_{i}(\vec{x})$, with $i \in\{1, \ldots, N\}$. $N$ is the number of elements in the basis as well as the dimension of the space $V^{h}$. Therefore, $p^{h}$ and $w^{h}$ may be represented as

$$
\begin{aligned}
& p^{h}(\vec{x})=\sum_{i=1}^{N} p_{i} \phi_{i}(\vec{x}) \\
& w^{h}(\vec{x})=\sum_{i=1}^{N} w_{i} \phi_{i}(\vec{x}) .
\end{aligned}
$$

Substituting these expressions into equation (3.17) and using the linearity of $a(\cdot, \cdot)$ and $\gamma(\cdot)$ results in the algebraic equation

$$
\vec{w} \cdot K \vec{p}=\vec{w} \cdot \vec{F} \quad \forall \vec{w} \in R^{N}
$$

The term $\vec{p}=\left(p_{1}, \ldots, p_{N}\right)^{T}$ is the vector components of $p^{h}$ in the basis $\phi_{i}$, and likewise for $\vec{w}$. This condition is satisfied if and only if the vector $\vec{p}$ is the solution of the matrix equation

$$
K \vec{p}=\vec{F}
$$

The components of the matrix $K$ and the vector $\vec{F}$ are defined as

$$
\begin{gathered}
K_{i j}=a\left(\phi_{i}, \phi_{j}\right), \\
\vec{F}_{i}=\gamma\left(\phi_{i}\right) \quad i, j \in\{1, \ldots, N\} .
\end{gathered}
$$

To solve equation (3.21), direct or iterative solvers are used (Golub and Van Loan, 1996). 
The finite element method discretizes the domain of a problem into small connected subdomains of some polygonal shape (usually triangles or tetrahedrons). This pattern of discretization is referred to as the domain's mesh. The individual subdomains within the mesh are known as the finite elements themselves. Each finite element is formed by nodes on its boundary, and elements are related by their shared nodes. If an element only has nodes at its vertices, it is said to be linear. Quadratic elements are formed by designating the midpoints of the line segment between the vertices as nodes. Even higher order elements may be formed with additional nodes, but their utility is limited with the techniques available (Becker et al., 1981).
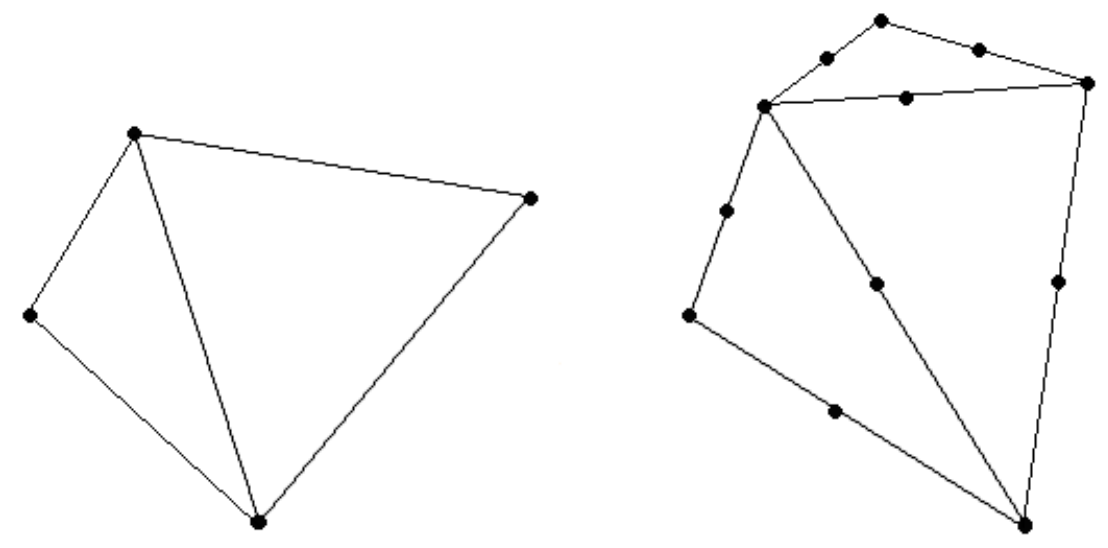

Figure 26 - Examples of two linear (left) and three quadratic (right) triangular finite elements, used to discretize two dimensional problems. The black dots represent the positions of the nodes.

In this work, quadratic triangular Lagrange elements were chosen for the discretization of the two dimensional domains and quadratic quadrilateral Lagrange elements for the three dimensional domains. Quadratic quadrilateral elements are composed of ten nodes, one for each of the four vertices and one on each midpoint. 


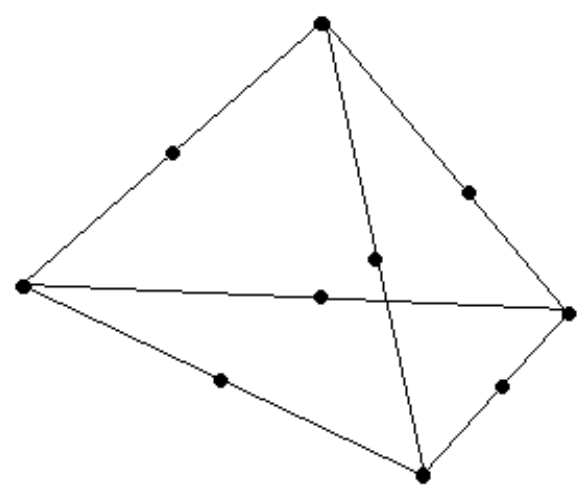

Figure 27 - Example of a three dimensional quadrilateral quadratic finite element. The black dots represent the positions of the ten nodes.

The basis functions are chosen based on the nodes of the problem. In this work, each basis function $\phi_{i}$ is a piecewise quadratic function, equal to one at node $i$ and equal to zero at all other nodes. With this choice of basis, the solutions for scattered pressure at the nodes are conveniently contained within the vector $\vec{p}$.

The major simplification in the above derivation concerns the outer boundary on which the simplified Sommerfeld radiation condition was assigned. The difficulty in solving scattering problems with the finite element lies with trying to reconcile infinity with a finite numerical domain. Because the goal in many cases, as it is here, is to understand the scattering from an object located in free space, away from boundaries and obstacles, scattering problems usually take place in an infinite domain. Since it is impossible to create an infinite numerical domain in the finite element method, such a problem must be simplified to only the scatterer and a finite region surrounding it. The scattered pressure can be determined anywhere within this surrounding region through the finite element method, but not outside of it. A technique for getting around this shortcoming is presented in the next section. 
The exterior of the surrounding medium region must be given a boundary condition that represents free space extending to infinity, known as a radiation condition. This boundary must model the effects of the free space it represents; specifically, it must not reflect outgoing waves back into the domain. Local absorbing boundary conditions (Atkinson, 1949; Wilcox, 1956), the Dirichlet to Neumann (DtN) boundary condition (Hunt, 1974), infinite elements (Bettess, 1977), and absorbing boundary layers (Berenger, 1994) are all techniques that seeks to eliminate this reflection. In the above derivation, a simplification of the Sommerfeld radiation condition was used. It is not the ideal choice for a radiation condition and can introduce errors into the calculation. However, as is seen in a later section, this radiation condition is necessary to solve the problem with COMSOL Multiphysics.

\subsection{Technique for Reducing Numerical Error in Two and Three Dimensions}

Increasing the number of elements and thus nodes in the solution process is one way to reduce the error in a finite element method approximation. Another method seeks to reduce the random numerical error after the solution process has taken place. This is possible in some applications of acoustic scattering due to the importance of point measurements. When determining an object's target strength, it is not necessary to solve for the scattered fields at all points. Equations (2.9) and (2.11) show how only a single point measurement of scattered pressure is needed. The finite element method approximates the solution at many locations, namely each node point. If the approximation is examined at one single node is used, there will be some numerical error present. However, if a great number of node point approximations can 
be interpreted to determine a single point's value, this numerical error can be diminished.

Techniques are now presented where a single value of scattered pressure at any point can be calculated from the knowledge of the field on a circle or a sphere surrounding the scatterer, in two and three dimensions respectively. The location of the calculated pressure is arbitrary outside of the immediate vicinity of the scatterer. This allows the scattered pressure to be determined at points well beyond the finite element method's numerical domain. In this way, scattered pressure can be determined in the far-field and used to calculate target strength.

\subsubsection{Circular Integral Method}

First, a polar coordinate system centered at the scatterer is defined with radial variable $r$ and polar variable $\varphi$. Start with the Helmholtz equation for the scattered field,

$$
\nabla^{2} p_{\text {scat }}+k^{2} p_{\text {scat }}=0
$$

The Laplacian operator in polar coordinates is defined as

$$
\nabla^{2}=\frac{1}{r} \frac{\partial}{\partial r}\left(r \frac{\partial}{\partial r}\right)+\frac{1}{r^{2}} \frac{\partial^{2}}{\partial \varphi^{2}}
$$

The Helmholtz equation is expanded to include the Laplacian,

$$
\frac{1}{r} \frac{\partial}{\partial r}\left(r \frac{\partial p_{\text {scat }}}{\partial r}\right)+\frac{1}{r^{2}} \frac{\partial^{2} p_{\text {scat }}}{\partial \varphi^{2}}+k^{2} p_{\text {scat }}=0
$$

Assume $p_{\text {scat }}$ is the sum of a unknown number of separable functions of $r$ and $\varphi$,

$$
p_{\text {scat }}(r, \varphi)=\sum_{\tau} \alpha_{\tau}(r) \beta_{\tau}(\varphi)
$$

A term in this series for $p_{\text {scat }}$ is inserted back into equation (3.25), and separation of variables is performed: 


$$
\frac{1}{\beta_{\tau}} \frac{\partial^{2} \beta_{\tau}}{\partial \varphi^{2}}=-\frac{r}{\alpha_{\tau}} \frac{\partial}{\partial r}\left(r \frac{\partial \alpha_{\tau}}{\partial r}\right)-k^{2} r^{2}=\delta_{\tau}
$$

where $\delta_{n}$ is a constant. Because of the requirement that $\beta_{\tau}(\varphi)=\beta_{\tau}(\varphi+2 \pi)$, the equation in $\varphi$ can be solved as

$$
\beta_{\tau}=C_{\tau} e^{i n \varphi}
$$

with

$$
\delta_{\tau}=-n^{2}
$$

where $n$ is any integer. The expression for $r$ in equation (3.27) becomes

$$
r^{2} \frac{\partial^{2} \alpha_{\tau}}{\partial r^{2}}+r \frac{\partial \alpha_{\tau}}{\partial r}+\alpha_{\tau}\left(k^{2} r^{2}-n^{2}\right)=0
$$

Performing a substitution of variables, $\xi=k r$, this becomes the well-known equation

$$
\xi^{2} \frac{\partial^{2} \alpha_{\tau}}{\partial \xi^{2}}+\xi \frac{\partial \alpha_{\tau}}{\partial \xi}+\alpha_{\tau}\left(\xi^{2}-n^{2}\right)=0
$$

whose solutions are the Bessel functions of the first and second kind, $J_{n}(\xi)$ and $Y_{n}(\xi)$

(Abramowitz and Stegun, 1965). Two linear independent combinations of these solutions are the Hankel functions of the first and second kind, $H_{n}^{(1)}(\xi)=J_{n}(\xi)+i Y_{n}(\xi)$ and $H_{n}^{(2)}(\xi)=J_{n}(\xi)-i Y_{n}(\xi)$

$$
\alpha_{\tau}=E_{i} H_{n}^{(1)}(k r)+F_{i} H_{n}^{(2)}(k r),
$$

where $E_{i}$ and $F_{i}$ are constants. Because $\alpha_{\tau}$ and $\beta_{\tau}$ are functions of integer $n$, the summation in equation becomes

$$
p_{\text {scat }}(r, \varphi)=\sum_{n=-\infty}^{\infty}\left[A_{n} H_{n}^{(1)}(k r)+B_{n} H_{n}^{(2)}(k r)\right] e^{i n \varphi}
$$

where $A_{n}$ and $B_{n}$ are constants. 
The n-dimensional Sommerfeld radiation condition (Sommerfeld, 1949) is given as,

$$
\lim _{r \rightarrow \infty}\left[r^{\frac{n-1}{2}}\left(\frac{\partial u}{d r}-i k u\right)\right]=0 .
$$

For the two dimensional scattered pressure, the condition becomes

$$
\lim _{r \rightarrow \infty}\left[\sqrt{r}\left(\frac{\partial p_{\text {scat }}}{d r}-i k p_{\text {scat }}\right)\right]=0 .
$$

Using the definition of the derivatives of the Hankel functions (Abramowitz and Stegun, 1965),

$$
\begin{gathered}
H_{n}^{(1)}(k r)^{\prime}=\frac{n}{k r} H_{n}^{(1)}(k r)-H_{n+1}^{(1)}(k r) \\
H_{n}^{(2)}(k r)^{\prime}=\frac{n}{k r} H_{n}^{(2)}(k r)-H_{n+1}^{(2)}(k r),
\end{gathered}
$$

the term in equation (3.35) is

$$
\sqrt{r}\left(\frac{\partial p_{\text {scat }}}{d r}-i k p_{\text {scat }}\right)=\sum_{n=-\infty}^{\infty} k \sqrt{r}\left[\begin{array}{c}
A_{n}\left(\frac{n}{k r} H_{n}^{(1)}(k r)-H_{n+1}^{(1)}(k r)-i H_{n}^{(1)}(k r)\right) \\
+B_{n}\left(\frac{n}{k r} H_{n}^{(2)}(k r)-H_{n+1}^{(2)}(k r)-i H_{n}^{(2)}(k r)\right)
\end{array}\right] e^{i n \varphi} .
$$

Taking the limit as $r \rightarrow \infty$,

$$
\begin{aligned}
H_{n}^{(1)}(k r) & \rightarrow \sqrt{\frac{2}{\pi k r}} e^{i\left(k r-\frac{n \pi}{2}-\frac{\pi}{4}\right)} \\
H_{n}^{(2)}(k r) & \rightarrow \sqrt{\frac{2}{\pi k r}} e^{-i\left(k r-\frac{n \pi}{2}-\frac{\pi}{4}\right)} \\
\lim _{r \rightarrow \infty}\left[\sqrt{r}\left(\frac{\partial p_{\text {scat }}}{d r}-i k p_{\text {scat }}\right)\right] & =\sum_{n=-\infty}^{\infty}\left[B_{n}\left(-2 \sqrt{\frac{2 k}{\pi}} e^{-i\left(k r-\frac{n \pi}{2}-\frac{3 \pi}{4}\right)}\right)\right] e^{i n \varphi} .
\end{aligned}
$$

To satisfy the Sommerfeld condition in equation (3.35), $B_{j n}=0$. The expression for scattered pressure becomes 


$$
p_{\text {scat }}(r, \varphi)=\sum_{n=-\infty}^{\infty} A_{n} H_{n}^{(1)}(k r) e^{i n \varphi}
$$

Now consider a circle of radius $R$ centered at the origin. If this circle completely surrounds the scatterer, then scattered pressure can be defined on it:

$$
p_{\text {scat }}(R, \varphi)=\sum_{n=-\infty}^{\infty} A_{n} H_{n}^{(1)}(k R) e^{i n \varphi}
$$

Multiplying both sides by $e^{-i m \varphi}$ and integrating over $\varphi$ around the circle,

$$
\begin{gathered}
\int_{0}^{2 \pi} p_{\text {scat }}(R, \varphi) e^{-i m \varphi} R d \varphi=\int_{0}^{2 \pi} \sum_{n=-\infty}^{\infty} A_{n} H_{n}^{(1)}(k R) e^{i n \varphi} e^{-i m \varphi} R d \varphi \\
\int_{0}^{2 \pi} p_{s c a t}(R, \varphi) e^{-i m \varphi} d \varphi=2 \pi A_{m} H_{m}^{(1)}(k R)
\end{gathered}
$$

Solving for the coefficient $A_{n}$,

$$
A_{n}=\frac{1}{2 \pi} \frac{1}{H_{n}^{(1)}(k R)} \int_{0}^{2 \pi} p_{s c a t}(R, \varphi) e^{-i n \varphi} d \varphi
$$

Therefore, the final expression for the scattered pressure at point $(r, \varphi)$ depends on an integral around a circle of radius $R$ :

$$
p_{\text {scat }}(r, \varphi)=\frac{1}{2 \pi} \sum_{n=-\infty}^{\infty} \frac{H_{n}^{(1)}(k r)}{H_{n}^{(1)}(k R)} e^{i n \varphi} \int_{0}^{2 \pi} p_{s c a t}\left(R, \varphi_{0}\right) e^{-i n \varphi_{0}} d \varphi_{0}
$$

\subsubsection{Spherical Integral Method}

In three dimensions, consider a scatterer located at the origin of at the origin of a $(r, \theta, \varphi)$ spherical coordinate system. Again start with the Helmholtz equation, (3.23). The Laplacian operator in spherical coordinates is defined as 


$$
\nabla^{2}=\frac{1}{r^{2}} \frac{\partial}{\partial r}\left(r^{2} \frac{\partial}{\partial r}\right)+\frac{1}{r^{2} \sin \theta} \frac{\partial}{\partial \theta}\left(\sin \theta \frac{\partial}{\partial \theta}\right)+\frac{1}{r^{2} \sin \theta^{2}} \frac{\partial^{2}}{\partial \varphi^{2}}
$$

Assume $p_{\text {scat }}$ is the sum of a number of separable functions of $r, \theta$, and $\varphi$ :

$$
p_{\text {scat }}(r, \theta, \varphi)=\sum_{\tau} \alpha_{\tau}(r) \beta_{\tau}(\theta) \gamma_{\tau}(\varphi)
$$

A term in this series is inserted back into the Helmholtz equation, and separation of variables is performed,

$$
\frac{1}{\gamma_{\tau}} \frac{\partial^{2} \gamma_{\tau}}{\partial \varphi^{2}}=-\frac{\sin ^{2} \theta}{\alpha_{\tau}} \frac{\partial}{\partial r}\left(r^{2} \frac{\partial \alpha_{\tau}}{\partial r}\right)-\frac{\sin \theta}{\beta_{\tau}} \frac{\partial}{\partial \theta}\left(\sin \theta \frac{\partial \beta_{\tau}}{\partial \theta}\right)-k^{2} r^{2} \sin ^{2} \theta=\varepsilon_{\tau}
$$

where $\varepsilon_{\tau}$ is a constant. Because of the condition $\gamma_{\tau}(\varphi)=\gamma_{\tau}(\varphi+2 \pi)$, the equation in $\varphi$ can be solved as

$$
\gamma_{\tau}=C_{\tau} e^{i j \varphi}
$$

where $C_{\tau}$ is a constant and $j$ is any integer, with

$$
\varepsilon_{\tau}=-j^{2}
$$

Inserting (3.51) into (3.49), separation of variables is performed again,

$$
\frac{1}{\beta_{\tau} \sin \theta} \frac{\partial}{\partial \theta}\left(\sin \theta \frac{\partial \beta_{\tau}}{\partial \theta}\right)-\frac{j^{2}}{\sin ^{2} \theta}=-\frac{1}{\alpha_{\tau}} \frac{\partial}{\partial r}\left(r^{2} \frac{\partial \alpha_{\tau}}{\partial r}\right)-k^{2} r^{2}=\delta_{\tau},
$$

where $\delta_{\tau}$ is a constant. Schwinger et al. (1998) give that the equation in $r$ is equal to the product

$$
\delta_{\tau}=-n(n+1)
$$

where $n$ is an integer. Substituting $x=\cos \theta$ into the equation for $\theta$ gives the well known equation

$$
\left(1-x^{2}\right) \frac{\partial^{2} \beta_{\tau}}{\partial x^{2}}-2 x \frac{\partial \beta_{\tau}}{\partial \theta}+\beta_{\tau}\left(n(n+1)-\frac{j^{2}}{1-x^{2}}\right)=0
$$


whose solutions are the Legendre functions of the first and second kind with degree $n$ and order $j, P_{n}^{j}(x)$ and $Q_{n}^{j}(x)$ (Abramowitz and Stegun, 1965). Because $x$ is only defined on the interval $[-1,1], Q_{n}^{j}(x)$ is undefined and $P_{n}^{j}(x)=P_{n}^{j}(\cos \theta)$ is the only solution. Therefore,

$$
\beta_{\tau}=D_{\tau} P_{n}^{j}(\cos \theta)
$$

where $D_{\tau}$ is a constant. The equation for $r$ in (3.52) becomes

$$
\frac{1}{\alpha_{\tau}} \frac{\partial}{\partial r}\left(r^{2} \frac{\partial \alpha_{\tau}}{\partial r}\right)+k^{2} r^{2}-n(n+1)=0 .
$$

A substitution $\xi=k r$ gives

$$
\xi^{2} \frac{\partial^{2} \alpha_{\tau}}{\partial \xi^{2}}+2 \xi \frac{\partial \alpha_{\tau}}{\partial \xi}+\alpha_{\tau}\left(\xi^{2}-n(n+1)\right)=0
$$

whose solutions of this differential equation are the spherical Bessel functions of the first and second kind, $j_{n}(\xi)$ and $y_{n}(\xi)$ (Abramowitz and Stegun, 1965). Two linear independent combinations of these are the spherical Hankel functions, $h_{n}^{(1)}(\xi)=j_{n}(\xi)+i y_{n}(\xi)$ and $h_{n}^{(2)}(\xi)=j_{n}(\xi)-i y_{n}(\xi):$

$$
\alpha_{\tau}=E_{\tau} h_{n}^{(1)}(k r)+F_{\tau} h_{n}^{(2)}(k r)
$$

with constants $E_{\tau}$ and $F_{\tau}$. Because $\alpha_{\tau}, \beta_{\tau}$, and $\gamma_{\tau}$ are functions of integers $j$ and $n$, the summation for scattered pressure in equation (3.48) becomes

$$
p_{\text {scat }}(r, \theta, \varphi)=\sum_{n=-\infty}^{\infty} \sum_{j=-\infty}^{\infty}\left(A_{j n} h_{n}^{(1)}(k r)+B_{j n} h_{n}^{(2)}(k r)\right) P_{n}^{j}(\cos \theta) e^{i j \varphi},
$$

where $A_{j n}$ and $B_{j n}$ are constants. Recall the three dimensional Sommerfeld radiation condition in equation (3.6). Using the definition of the derivatives of spherical Hankel functions, 


$$
\begin{gathered}
h_{n}^{(1)}(k r)^{\prime}=\frac{n}{k r} h_{n}^{(1)}(k r)-h_{n+1}^{(1)}(k r) \\
h_{n}^{(2)}(k r)^{\prime}=\frac{n}{k r} h_{n}^{(2)}(k r)-h_{n+1}^{(2)}(k r),
\end{gathered}
$$

the term in the limit of the Sommerfeld condition becomes

$$
\begin{gathered}
r\left(\frac{\partial p_{s c a t}}{d r}-i k p_{s c a t}\right)= \\
\sum_{j=-\infty}^{\infty} \sum_{n=-\infty}^{\infty} k r\left(\begin{array}{c}
A_{j n}\left[\frac{n}{k r} h_{n}^{(1)}(k r)-h_{n+1}^{(1)}(k r)-i h_{n}^{(1)}(k r)\right] \\
+B_{j n}\left[\frac{n}{k r} h_{n}^{(2)}(k r)-h_{n+1}^{(2)}(k r)--i h_{n}^{(2)}(k r)\right]
\end{array}\right) P_{n}^{j}(\cos \theta) e^{i j \varphi} .
\end{gathered}
$$

Taking the limit $r \rightarrow \infty$,

$$
\begin{gathered}
h_{n}^{(1)}(k r) \rightarrow \frac{1}{k r} e^{i\left(k r-\frac{n \pi}{2}-\frac{\pi}{2}\right)} \\
h_{n}^{(2)}(k r) \rightarrow \frac{1}{k r} e^{-i\left(k r-\frac{n \pi}{2}-\frac{\pi}{2}\right)} \\
\lim _{r \rightarrow \infty}\left[r\left(\frac{\partial p_{s c a t}}{d r}-i k p_{s c a t}\right)\right]=\sum_{j=-\infty}^{\infty} \sum_{n=-\infty}^{\infty}\left(B_{j n}\left[-2 e^{-i\left(k r-\frac{n \pi}{2}-\pi\right)}\right]\right) P_{n}^{j}(\cos \theta) e^{i j \varphi}
\end{gathered}
$$

To satisfy the Sommerfeld condition, $B_{j n}=0$. Equation (3.59) becomes

$$
p_{s c a t}(r, \theta, \varphi)=\sum_{n=-\infty}^{\infty} \sum_{j=-\infty}^{\infty} A_{j n} h_{n}^{(1)}(k r) P_{n}^{j}(\cos \theta) e^{i j \varphi}
$$

Legendre functions follow two important recurrence relations (Abramowitz and Stegun, 1965):

$$
\begin{gathered}
P_{v}^{\mu+1}(z)=\left(z^{2}-1\right)^{-1 / 2}\left[(v-\mu) z P_{v}^{\mu}(z)-(v+\mu) P_{v-1}^{\mu}(z)\right] \\
P_{-v-1}^{\mu}=P_{v}^{\mu} .
\end{gathered}
$$


These allow any Legendre function $P_{n}^{j}$ to be written as a combination of others with both $n$ positive and $j \in[-n,-n+1 \ldots n-1, n]$. This simplifies the summation limits in the expression for scattered pressure:

$$
p_{\text {scat }}(r, \theta, \varphi)=\sum_{n=0}^{\infty} \sum_{j=-n}^{n} A_{j n} h_{n}^{(1)}(k r) P_{n}^{j}(\cos \theta) e^{i j \varphi}
$$

Now consider a sphere of radius $R$ centered at the origin with coordinates $\left(R, \theta_{0}, \varphi_{0}\right)$. If this completely surrounds the scatterer, the scattered pressure can be defined on it:

$$
p_{\text {scat }}\left(R, \theta_{0}, \varphi_{0}\right)=\sum_{n=0}^{\infty} \sum_{j=-n}^{n} A_{j n} h_{n}^{(1)}(k R) P_{n}^{j}\left(\cos \theta_{0}\right) e^{i j \varphi_{0}}
$$

Multiply both sides by $P_{\hat{n}}^{\hat{j}}\left(\cos \theta_{0}\right) e^{-\hat{i} \hat{j} \varphi_{0}}$, where $\hat{n}$ and $\hat{j}$ are each some integer,

$$
\begin{gathered}
p_{\text {scat }}\left(R, \theta_{0}, \varphi_{0}\right) P_{\hat{n}}^{\hat{j}}\left(\cos \theta_{0}\right) e^{-\hat{i} \hat{\varphi} \varphi_{0}}= \\
\sum_{n=0}^{\infty} \sum_{j=-n}^{n} A_{j n} h_{n}^{(1)}(k R) P_{n}^{j}\left(\cos \theta_{0}\right) e^{i j \varphi_{0}} P_{\hat{n}}^{\hat{j}}\left(\cos \theta_{0}\right) e^{-\hat{i} \varphi_{0}} .
\end{gathered}
$$

This expression is then integrated over the surface of the sphere,

$$
\begin{gathered}
\int_{0}^{\pi} \int_{0}^{2 \pi} p_{\text {scat }}\left(R, \theta_{0}, \varphi_{0}\right) P_{\hat{n}}^{\hat{j}}\left(\cos \theta_{0}\right) e^{-\hat{i} \varphi_{0}} R^{2} \sin \theta_{0} d \varphi_{0} d \theta_{0} \\
=\int_{0}^{\pi} \int_{0}^{2 \pi} \sum_{n=0}^{\infty} \sum_{j=-n}^{n} A_{j n} h_{n}^{(1)}(k R) P_{n}^{j}\left(\cos \theta_{0}\right) P_{\hat{n}}^{\hat{j}}\left(\cos \theta_{0}\right) e^{i(j-\hat{j}) \varphi_{0}} R^{2} \sin \theta_{0} d \varphi_{0} d \theta_{0} .
\end{gathered}
$$

The integral over $\varphi_{0}$ on the right is zero when $j \neq \hat{j}$ and $2 \pi$ when $j=\hat{j}$. Therefore the expression simplifies to

$$
\int_{0}^{\pi} \int_{0}^{2 \pi} p_{\text {scat }}\left(R, \theta_{0}, \varphi_{0}\right) P_{\hat{n}}^{\hat{j}}\left(\cos \theta_{0}\right) e^{-\hat{i} \hat{\varphi} \varphi_{0}} \sin \theta_{0} d \varphi_{0} d \theta_{0}
$$




$$
=2 \pi \int_{0}^{\pi} \sum_{n=0}^{\infty} A_{\hat{j} n} h_{n}^{(1)}(k R) P_{n}^{\hat{j}}\left(\cos \theta_{0}\right) P_{\hat{n}}^{\hat{j}}\left(\cos \theta_{0}\right) \sin \theta_{0} d \theta_{0} .
$$

A substitution of variables $\cos \theta_{0}=x$ into the right side of the expression gives

$$
\int_{0}^{\pi} \int_{0}^{2 \pi} p_{\text {scat }}\left(R, \theta_{0}, \varphi_{0}\right) P_{\hat{n}}^{\hat{j}}\left(\cos \theta_{0}\right) e^{-\hat{i} \hat{\varphi} \varphi_{0}} \sin \theta_{0} d \varphi_{0} d \theta_{0}=2 \pi \int_{-1}^{1} \sum_{n=0}^{\infty} A_{\hat{j} n} h_{n}^{(1)}(k R) P_{n}^{\hat{j}}(x) P_{\hat{n}}^{\hat{j}}(x) d x .
$$

Legendre functions of the same degree and different order are orthogonal on the interval $[-1,1]$ (Abramowitz and Stegun, 1965):

$$
\int_{-1}^{1} P_{b}^{a}(x) P_{c}^{a}(x) d x=0
$$

when $b \neq c$. Therefore, the expression becomes

$$
\int_{0}^{\pi} \int_{0}^{2 \pi} p_{\text {scat }}\left(R, \theta_{0}, \varphi_{0}\right) P_{\hat{n}}^{\hat{j}}\left(\cos \theta_{0}\right) e^{-\hat{i} \varphi_{0}} \sin \theta_{0} d \varphi_{0} d \theta_{0}=A_{\hat{j} \hat{n}} 2 \pi h_{\hat{n}}^{(1)}(k R) \int_{-1}^{1} P_{\hat{n}}^{\hat{j}}(x)^{2} d x
$$

Now $A_{j n}$ can be solved for,

$$
A_{j n}=\frac{\int_{0}^{\pi} \int_{0}^{2 \pi} p_{s c a t}\left(R, \theta_{0}, \varphi_{0}\right) P_{n}^{j}\left(\cos \theta_{0}\right) e^{-i j \varphi_{0}} \sin \theta_{0} d \varphi_{0} d \theta_{0}}{2 \pi h_{n}^{(1)}(k R) \int_{-1}^{1} P_{n}^{j}(x)^{2} d x}
$$

Two cases must be considered: $j \geq 0$ and $j<0$. When $j \geq 0$, there is an expression for the integral in the denominator of (3.76) (Abramowitz and Stegun, 1965):

$$
\int_{-1}^{1} P_{n}^{j}(x)^{2} d x=\frac{(n+j) !}{(n+1 / 2)(n-j) !} .
$$

Inserting this back into the expressions for $A_{j n}$ and scattered pressure in (3.68), 


$$
\begin{gathered}
A_{j n}=\frac{\int_{0}^{\pi} \int_{0}^{2 \pi} p_{\text {scat }}\left(R, \theta_{0}, \varphi_{0}\right) P_{n}^{j}\left(\cos \theta_{0}\right) e^{-i j \varphi_{0}} \sin \theta_{0} d \varphi_{0} d \theta_{0}(n+1 / 2)(n-j) !}{2 \pi h_{n}^{(1)}(k R)(n+j) !} \\
p_{\text {scat }}(r, \theta, \varphi)=\frac{1}{2 \pi} \sum_{n=0}^{\infty} \sum_{j=1}^{n} \frac{(n+1 / 2)(n-j) ! h_{n}^{(1)}(k r) P_{n}^{j}(\cos \theta) e^{i j \varphi}}{(n+j) ! h_{n}^{(1)}(k R)} \\
\quad \times \int_{0}^{\pi} \int_{0}^{2 \pi} p_{\text {scat }}\left(R, \theta_{0}, \varphi_{0}\right) P_{n}^{j}\left(\cos \theta_{0}\right) e^{-i j \varphi_{0}} \sin \theta_{0} d \varphi_{0} d \theta_{0} .
\end{gathered}
$$

The second case is when $j<0$. Legendre functions of negative order can be written as

$$
P_{n}^{j}(x)=\frac{(n+j+1) !}{(n-j+1) !} P_{n}^{-j}(x)
$$

Equation (3.76) can be rewritten as

$$
A_{j n}=\frac{\int_{0}^{\pi} \int_{0}^{2 \pi} p_{s c a t}\left(R, \theta_{0}, \varphi_{0}\right) P_{n}^{j}\left(\cos \theta_{0}\right) e^{-i j \varphi_{0}} \sin \theta_{0} d \varphi_{0} d \theta_{0}}{2 \pi h_{n}^{(1)}(k R) \int_{-1}^{1}\left[\frac{(n+j+1) !}{(n-j+1) !} P_{n}^{-j}(x)\right]^{2} d x}
$$

Now the integral in the denominator can be evaluated:

$$
A_{j n}=\frac{\int_{0}^{\pi} \int_{0}^{2 \pi} p_{\text {scat }}\left(R, \theta_{0}, \varphi_{0}\right) P_{n}^{j}\left(\cos \theta_{0}\right) e^{-i j \varphi_{0}} \sin \theta_{0} d \varphi_{0} d \theta_{0}}{2 \pi h_{n}^{(1)}(k R)\left(\frac{(n+j+1) !}{(n-j+1) !}\right)^{2} \frac{(n-j) !}{(n+1 / 2)(n+j) !}}
$$

Plugging this back into the expression for scattered pressure (3.68),

$$
\begin{gathered}
p_{\text {scat }}(r, \theta, \varphi)=\frac{1}{2 \pi} \sum_{n=0}^{\infty} \sum_{j=-n}^{n} \frac{(n+1 / 2)(n+j) ! h_{n}^{(1)}(k r) P_{n}^{j}(\cos \theta) e^{i j \varphi}}{(n-j) !\left(\frac{(n+j+1) !}{(n-j+1) !}\right)^{2} h_{n}^{(1)}(k R)} \\
\quad \times \int_{0}^{\pi} \int_{0}^{2 \pi} p_{\text {scat }}\left(R, \theta_{0}, \varphi_{0}\right) P_{n}^{j}\left(\cos \theta_{0}\right) e^{-i j \varphi_{0}} \sin \theta_{0} d \varphi_{0} d \theta_{0} .
\end{gathered}
$$

Using the expression in (3.80), this becomes 


$$
\begin{gathered}
p_{\text {scat }}(r, \theta, \varphi)=\frac{1}{2 \pi} \sum_{n=0}^{\infty} \sum_{j=-n}^{n} \frac{(n+1 / 2)(n+j) ! h_{n}^{(1)}(k r) P_{n}^{-j}(\cos \theta) e^{i j \varphi}}{(n-j) ! h_{n}^{(1)}(k R)} \\
\quad \times \int_{0}^{\pi} \int_{0}^{2 \pi} p_{\text {scat }}\left(R, \theta_{0}, \varphi_{0}\right) P_{n}^{-j}\left(\cos \theta_{0}\right) e^{-i j \varphi_{0}} \sin \theta_{0} d \varphi_{0} d \theta_{0} .
\end{gathered}
$$

Comparing (3.79) when $j \geq 0$ to (3.84) when $j<0$, it is clear the expression for scattered pressure may be written in one form for all $j$ :

$$
\begin{gathered}
p_{\text {scat }}(r, \theta, \varphi)=\frac{1}{2 \pi} \sum_{n=0}^{\infty} \sum_{j=-n}^{n} \frac{(n+1 / 2)(n-|j|) ! h_{n}^{(1)}(k r) P_{n}^{|j|}(\cos \theta) e^{i j \varphi}}{(n+|j|) ! h_{n}^{(1)}(k R)} \\
\quad \times \int_{0}^{\pi} \int_{0}^{2 \pi} p_{\text {scat }}\left(R, \theta_{0}, \varphi_{0}\right) P_{n}^{|j|}\left(\cos \theta_{0}\right) e^{-i j \varphi_{0}} \sin \theta_{0} d \varphi_{0} d \theta_{0}
\end{gathered}
$$

This equation can be written so that the surface integral is more general:

$$
\begin{aligned}
& p_{\text {scat }}(r, \theta, \varphi)=\sum_{n=0}^{\infty} \sum_{j=-n}^{n} \frac{h_{n}^{(1)}(k r)}{h_{n}^{(1)}(k R)} \Phi_{j n}(R, \theta, \varphi) \int_{\Gamma} \Phi_{j n}\left(R, \theta_{0}, \varphi_{0}\right) * p_{s c a t}\left(R, \theta_{0}, \varphi_{0}\right) d \Gamma \\
& \text { and } \quad \Phi_{j n}(R, \theta, \varphi)=\sqrt{\frac{(2 n+1)(n-|j|) !}{4 \pi R^{2}(n+|j|) !}} P_{n}^{|j|}(\cos \theta) e^{i j \varphi},
\end{aligned}
$$

where $\Gamma$ is the surface of the sphere and * denotes the complex conjugate.

Equations (3.46) and (3.86) give the expressions for scattered pressure at any point in two and three dimensions, based on the knowledge of the field on a circle and a sphere surrounding the scatterer respectively. These expressions are very helpful in conjunction with the finite element method, which solves for the scattered pressure over a finite domain around the object. The scattered pressure can be ascertained on a circle or sphere from the finite element method, and then used to find its value at any point by the above techniques. In addition, they prove to help remove significant amounts of numerical error. 


\subsection{Implementation of COMSOL Multiphysics}

COMSOL Multiphysics (ver. 3.2a) was used in this work for the finite element method simulations.

\subsubsection{COMSOL Methodology}

\subsubsection{Geometry and Elements}

The process of creating scatterer geometry within COMSOL was discussed when solving the Kirchhoff integral numerically. In finite element method scattering problems, the surrounding medium subdomain must be created as well. To simplify the implementation of the radiation condition on the boundary, this exterior region is modeled as a sphere in three dimensions and a circle in two. The radius of this sphere (or circle) is labeled as $A$. To help visualize, figure 28 shows two sample finite element domains in COMSOL. The sphere and circle represent the surrounding medium subdomains, while the box and square represent the scatterer subdomains. 

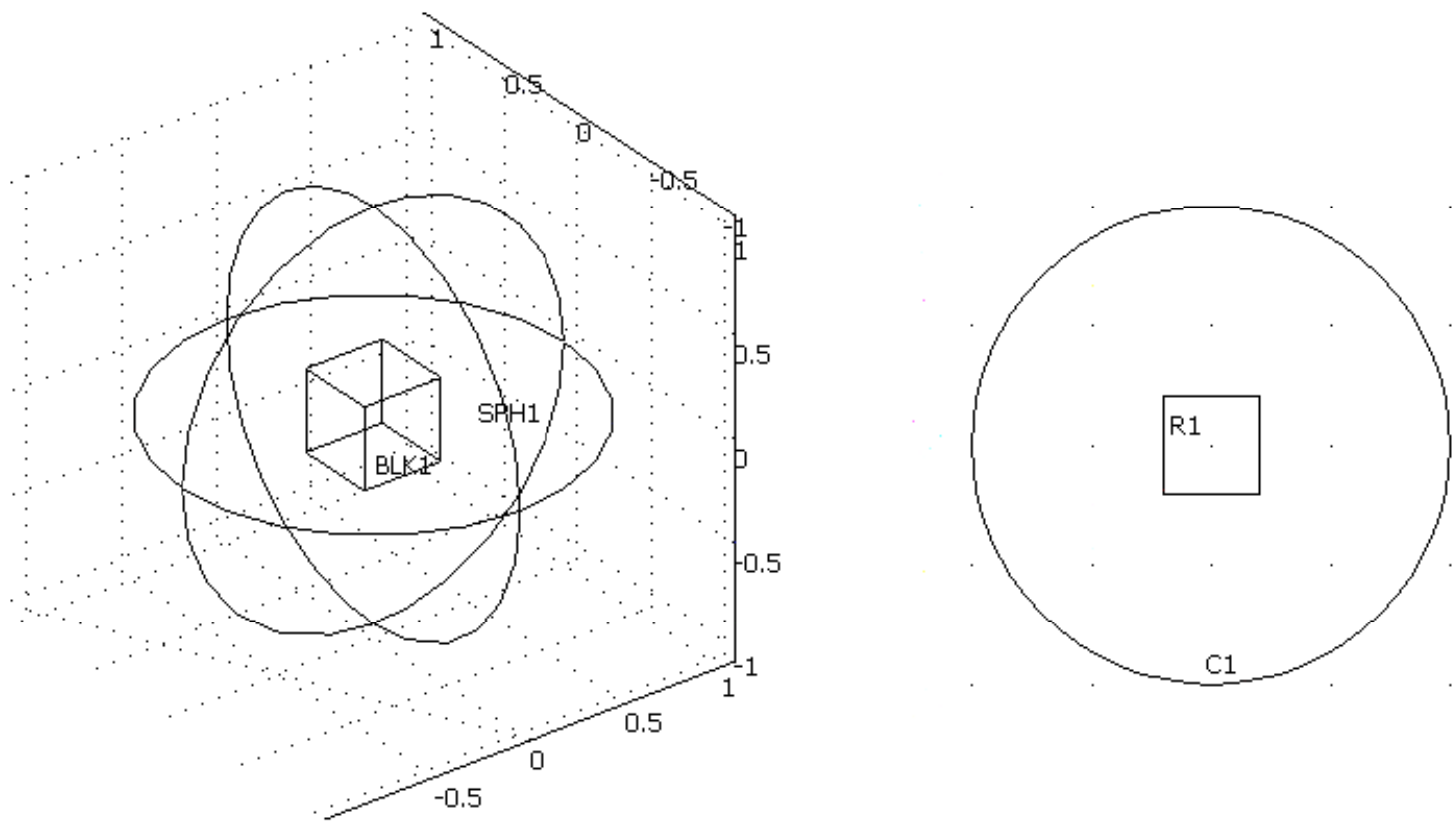

Figure 28 - Sample three dimensional and two dimensional finite element domains in COMSOL.

After the geometries of these two subdomains - scatterer and surrounding medium - are defined, the elements themselves can be created. Identical to the process in the Kirchhoff chapter, this consists of discretizing the domain into quadrilaterals (or triangles in two dimensions). In this application, the size of the elements is again important to the accuracy of the solution. Elements may be defined by a maximum allowable size, $h_{\max }$. The effects of element size will be explored shortly.

\subsubsection{Defining the Partial Differential Equations}

The next step is to create the partial differential equations that are used in the numerical calculation. This is done by defining the coefficients of the generic partial differential equations used in COMSOL. Every subdomain and boundary between subdomains requires an equation. In the case of acoustic scattering, the subdomain equations describe the traveling waves in 
terms of total pressure, the sum of incident and scattered fields. Boundary equations are used to represent both the boundary condition between the scatterer and surrounding medium and the radiation condition at the edge of the numerical domain.

The general equation for subdomains in COMSOL is

$$
\nabla \cdot(-c \nabla u-\alpha u+\gamma)+a u+\beta \cdot \nabla u=f
$$

where the coefficients must be defined. Let $u$ represent the total pressure:

$$
u=p_{\text {tot }}=p_{\text {inc }}+p_{\text {scat }} \text {. }
$$

From COMSOL's solution for total pressure, scattered pressure is easily solved for by subtracting out the known incident field. To describe the pressure field, equation (3.87) should model the Helmholtz equation (3.1). This is easily done by configuring the coefficients as follows:

$$
\begin{aligned}
& c=1 \\
& \alpha=0 \\
& \gamma=0 \\
& a=-k^{2} \\
& \beta=0 \\
& f=0
\end{aligned}
$$

Terms like $k$ can either have their values entered explicitly into the equations, or they can be defined separately to allow for symbolic equations.

The first boundary condition that has to be described is between the scatterer and the surrounding medium. This can be modeled as a variety of different boundaries: rigid/fixed, soft, fluid, and potentially elastic (although this was not tested). COMSOL provides two generic equations that can be used to model a Neumann or Dirichlet boundary condition,

$$
\hat{n} \cdot(c \nabla u+\alpha u-\gamma)+q u=g-h^{\mathrm{T}} \mu
$$




$$
h u=r,
$$

where $\hat{n}$ is the outward normal vector from the surface and $\mu$ is a Lagrange multiplier. Several of the coefficients here $(c, \alpha, \gamma)$ are already defined in the subdomain description in (3.89). Substituting these values and rewriting,

$$
\begin{gathered}
\hat{n} \cdot \nabla u+q u=g-h^{\mathrm{T}} \mu \\
h u=r .
\end{gathered}
$$

By manipulating the coefficients of this equation, all of the necessary boundary conditions can be replicated. For example, in a rigid/fixed boundary, the normal gradient of pressure is zero on the boundary. To model this, a Neumann boundary condition is used and all remaining coefficients are set to zero:

$$
\begin{gathered}
\hat{n} \cdot \nabla u=0 \\
0=0 .
\end{gathered}
$$

For a soft boundary condition, the pressure goes to zero at the interface. To model this, $h$ is set at a nonzero value in order to include the second equation. The simplest method is to set it equal to one. Then because of the Lagrange multiplier, the first equation imposes no restriction on $u$ and can thereby be ignored. The second equation simply ends up being

$$
u=r
$$

and $r$ can be set to any value; this is zero in the case of the soft boundary condition. Similar steps can be taken to create a fluid boundary, requiring continuity of both pressure and pressure gradient at the interface. In this work, the rigid/fixed condition was always used.

The need to model free space at the boundary of the finite surrounding medium subdomain in acoustic scattering problems has been discussed. One way to do this is to use a 
simplified version of the Sommerfeld radiation condition, introduced in a previous section as equation (3.7),

$$
\frac{\partial p_{s c a t}}{\partial r}=i k p_{s c a t}
$$

This is an equation for the scattered field, not the total field for which COMSOL solves.

Therefore, the scattered field must be written in terms of the total field and the known incident field,

$$
p_{\text {scat }}=u-p_{\text {inc }}
$$

The simplified Sommerfeld condition becomes

$$
\hat{n} \cdot \nabla\left(u-p_{i n c}\right)=i k\left(u-p_{i n c}\right) .
$$

The incident field must now be defined. For an incident plane wave of amplitude $p_{0}$ and wave vector $\vec{k}$ at position $\vec{r}$,

$$
p_{i n c}=p_{0} e^{i(\vec{k} \cdot \vec{r})}
$$

Inserting this into equation (3.96) and rearranging terms,

$$
\hat{n} \cdot \nabla u-i k u=i[(\hat{n} \cdot \vec{k})-k] p_{0} e^{i(\vec{k} \cdot \vec{r})}
$$

Looking again at COMSOL's equations in (3.90), this condition can be represented by configuring the coefficients as follows:

$$
\begin{aligned}
q & =-i k \\
g & =i[(\hat{n} \cdot \vec{k})-k] p_{0} e^{i(\vec{k} \cdot \vec{r})} \\
h & =0 \\
r & =0
\end{aligned}
$$




\subsubsection{The Solution Phase}

The large matrix equations are automatically compiled by COMSOL and run through one of its linear solvers. For two-dimensional problems, the UMFPACK solver was used. This solves the matrix equations directly using LU factorization. For the three-dimensional problems requiring much more memory, the GMRES iterative solver was used instead. While a slower solver than UMFPACK, GMRES allowed for calculations with many more elements.

\subsubsection{Accuracy of the Solution}

The accuracy of the finite element method approximation can be judged several ways. It can simply be interpreted at a single node point as the difference between the exact solution and the computed value,

$$
e(x)=u(x)-u_{F E M}(x)
$$

There are a variety of error norms that give a more global understanding of a solution's accuracy. Two examples are the energy and maximum norms (Becker et al., 1981),

$$
\begin{gathered}
\|e\|_{E}=\left\{\int_{\Omega}\left[\left(e(x)^{\prime}\right)^{2}+e(x)^{2}\right] d \Omega\right\}^{1 / 2}, \\
\|e\|_{\infty}=\max _{x \in \Omega}|e(x)| .
\end{gathered}
$$

Whenever using the finite element method (or any numerical technique), error in the solution is expected as a result of simply discretizing the domain. Other error effects are specific to the problem; in this case, it is the use of the simplified Sommerfeld radiation condition. In order to help understand and attempt to separate the effects from the discretization and the radiation condition, two unitless parameters were created: $E P W$ and $S M D$. 
Given a mesh of elements with maximum element size $h_{\max }$ for a problem with wavenumber $k$,

$$
E P W=\frac{\lambda}{h_{\max }}=\frac{2 \pi}{h_{\max } k}
$$

In this way, there are at least $E P W$ elements per wavelength. By increasing $E P W$, the elements will get smaller relative to wavelength. Therefore this parameter is a measure of the discretization, generally with larger values resulting in more accurate solutions.

For a spherical surrounding medium domain of radius $A$ centered at the origin, with a scatterer whose maximum extension from the origin is distance $r_{\max }^{\prime}$, and with wavenumber $k$, define

$$
S M D=k\left(A-r_{\max }^{\prime}\right)
$$

This parameter is the minimum distance a scattered wave must travel before it reaches the outer boundary of the surrounding medium in terms of wavenumber. Because the simplified

Sommerfeld radiation condition has an error of order $o\left(\frac{1}{r}\right)$, increasing SMD should increasing the accuracy of the solution.

\subsubsection{Two Dimensional Test: Rigid/Fixed Infinite Cylinder (Max Error Norm)}

The finite element method with the simplified Sommerfeld condition can be tested for several problems with known solutions. A simple problem that can be tested is scattering from a rigid/fixed infinite cylinder, which has a known scattering solution that does not vary along its length (Rayleigh, 1945). For an incident plane wave $p_{i n c}=p_{0} e^{i k x}$, 


$$
\begin{aligned}
& \qquad p_{s c a t}=p_{0} \sum_{m=0}^{\infty} B_{m} \cos (m \varphi) H_{m}^{(1)}(k r) \\
& \text { and } \quad B_{m}=\frac{-\varepsilon_{m} i^{m} J_{m}(k a)^{\prime}}{H_{m}^{(1)}(k a)^{\prime}},
\end{aligned}
$$

where $\varepsilon_{m}$ is equal to 1 for $m=0$ and equal to 2 for all other vales of $m$. Because of the symmetry of the infinite cylinder, this problem may be thought of as the scattering from a rigid/fixed circle in two dimensions.

The two dimensional problem can be run in COMSOL, and the approximations at each node can be compared to the exact solutions at those points. Assume an infinite cylinder lies along the $\mathrm{z}$ axis with radius $a$. The cylinder experiences an incident field of $e^{i k x}$, representing a plane wave traveling in the positive $\mathrm{x}$ direction with amplitude $p_{0}=1$ and wavenumber $k$. To model the problem in COMSOL, a circle of radius $a=1$ centered at the origin represents the cylinder. Because of symmetry,

$$
S M D=k(A-a)
$$

This parameter was varied over several values and was used to determine $A$, the radius of the outer domain. The parameter $E P W$ was varied from two to eight, representing the minimum number of elements per wavelength. The solution was generated in COMSOL and the scattered field was determined at each node point. 

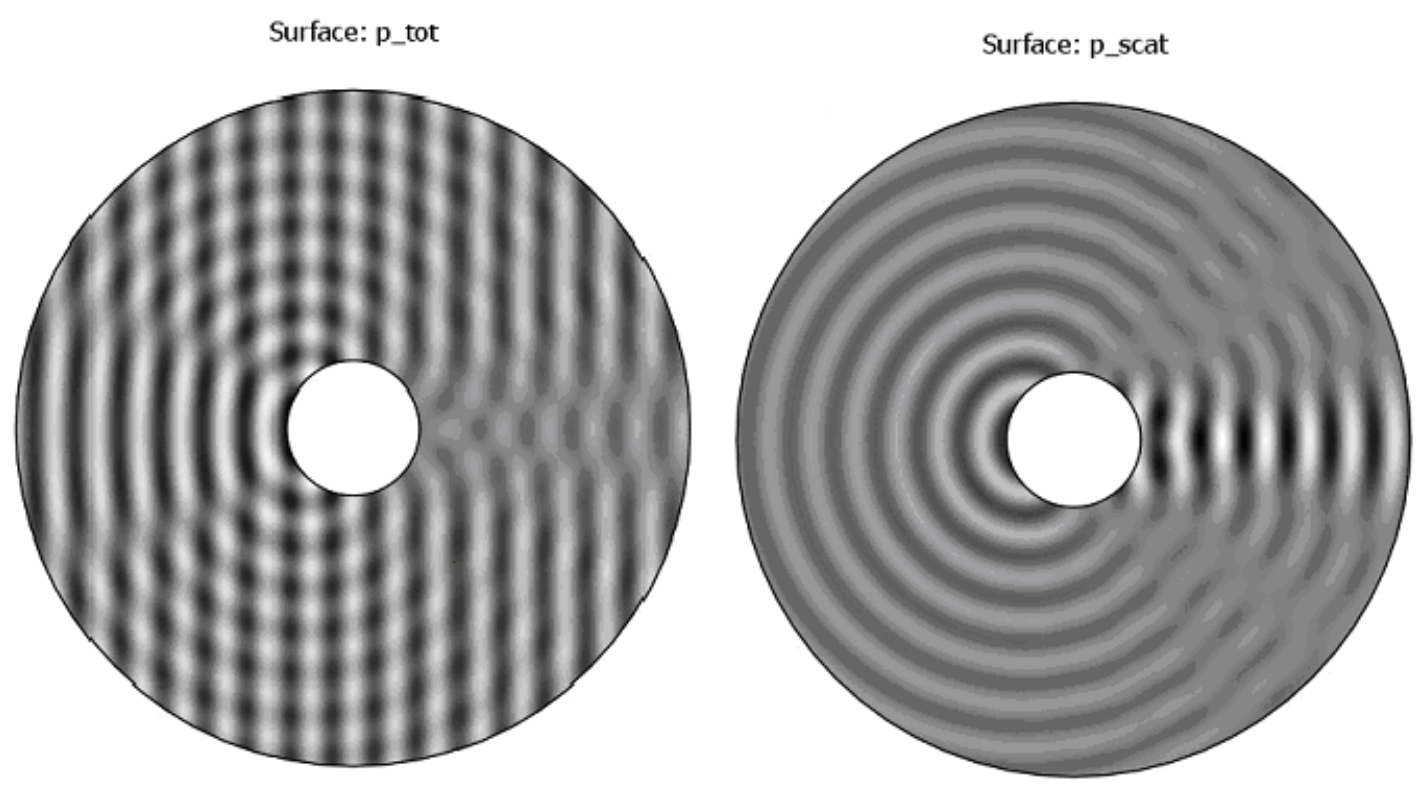

Figure 29 - Total and scattered pressure fields from a rigid/fixed infinite cylinder, $k a=10$ in COMSOL. The incident wave arrives from the left side of the domain.

The maximum error norm was determined by comparing the solutions at every node and finding the maximum deviation from the exact solution. This was done at two separate frequencies, determined by ka, the product of the wavenumber and the cylinder's radius. 


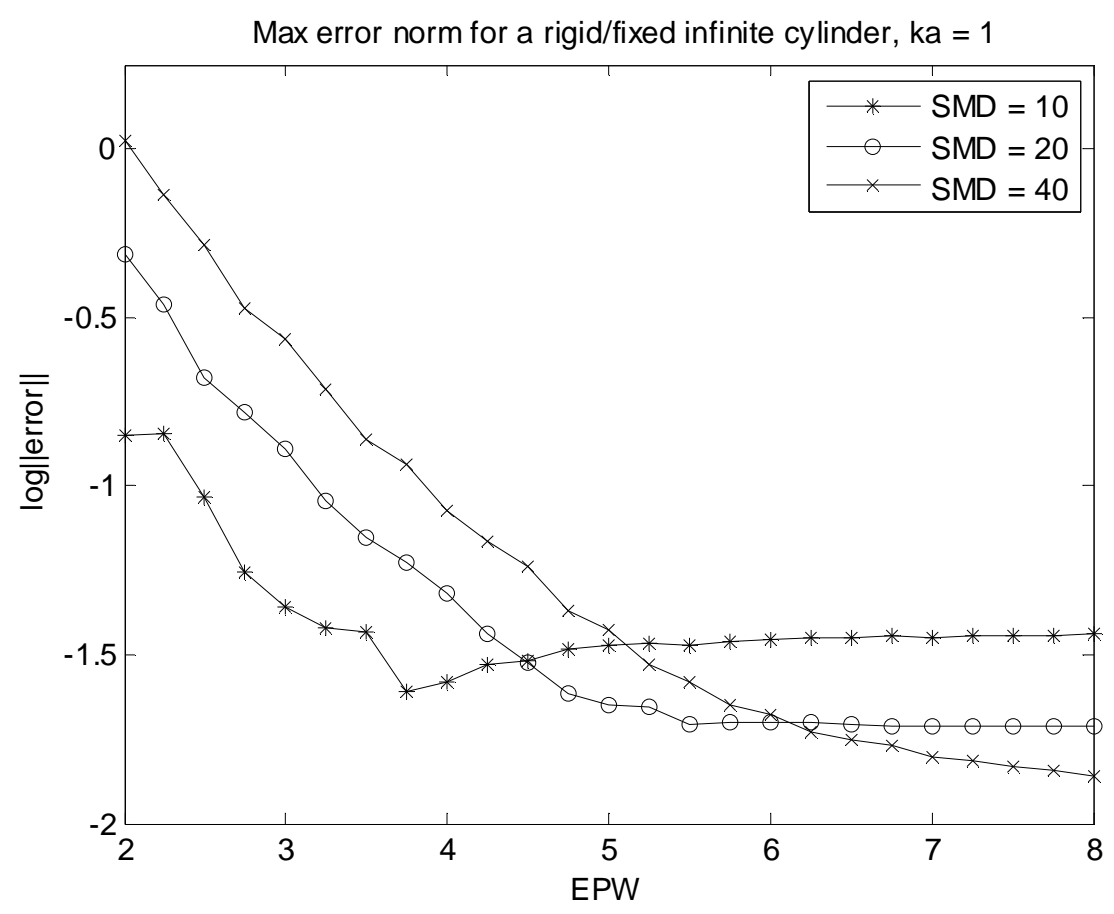

Figure 30 - Max error norm versus EPW for COMSOL approximation of scattering from a rigid/fixed infinite cylinder at $\mathbf{k a}=\mathbf{1}$.

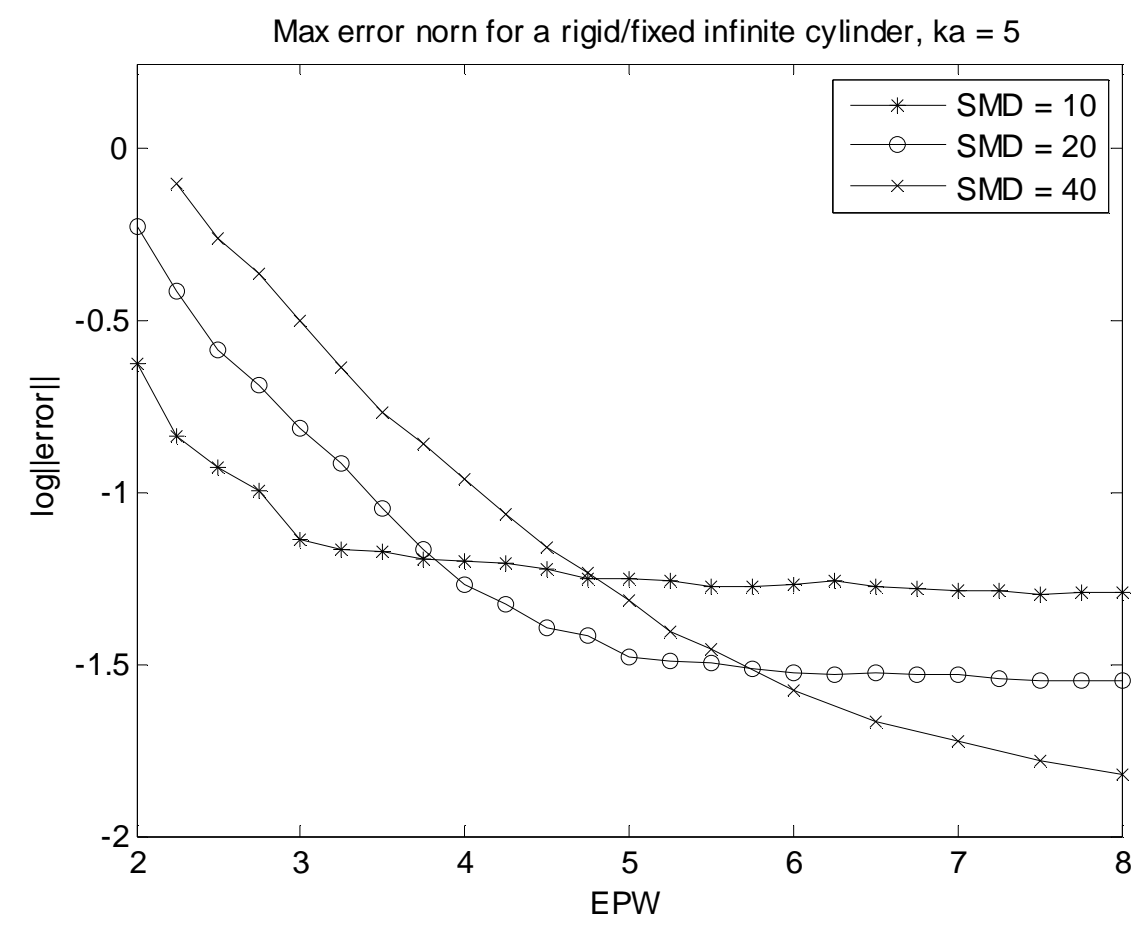

Figure 31 - Max error norm versus EPW for COMSOL approximation of scattering from a rigid/fixed infinite cylinder at $\mathbf{k a}=5$. 
As expected, decreasing element size also decreases the error norm of the solution. The error slows its reduction and in some cases stops it as when the elements reach a certain size. The error that remains for the curves that have leveled out is a result of the radiation condition used and cannot be fixed by discretizing the domain further. As expected, within this range of small element size, domain size is a key factor in the amount of error in the solution. Increasing the domain size helps to reduce error when further discretization has no effect.

\subsubsection{Two Dimensional Test: Rigid/Fixed Infinite Cylinder (Infinite Form Function)}

Because target strength is undefined for infinite objects, the infinite form function $f^{\infty}$ can be used for comparison instead. Similar to the scattering amplitude of a finite object, the infinite form function of a cylinder of radius $a$ is defined only in the far-field $k r>>1$ (Stanton, 1992):

$$
p_{\text {scat }} \approx p_{0} \sqrt{\frac{a}{2 r}} e^{i k r} f^{\infty} .
$$

For a cylinder aligned with the $\mathrm{z}$ axis, the infinite form function for a rigid/fixed cylinder from an incident plane wave $p_{i n c}=p_{0} e^{i k x}$ is defined as

$$
\begin{gathered}
f^{\infty}=\frac{2}{\sqrt{\pi k a}} e^{-i \pi / 4} \sum_{m=0}^{\infty} b_{m} \cos (m \varphi) \\
\text { and } \quad b_{m}=\frac{-\varepsilon_{m} J_{m}(k a)^{\prime}}{H_{m}^{(1)}(k a)^{\prime}} .
\end{gathered}
$$

In the backscattering direction, $\varphi=\pi$, giving

$$
f_{b s}^{\infty}=\frac{2}{\sqrt{\pi k a}} e^{-i \pi / 4} \sum_{m=0}^{\infty} b_{m}(-1)^{m}
$$

Again, the only interest here is in this backscattering direction, so the subscript will be dropped. 
The value of scattered pressure used to find the infinite form function in equation (3.108) can be determined two ways from COMSOL's solution. It can either be taken as a simple point measurement from the solution itself, or it can be calculated using the circular integral method in equation (3.46). This latter method was used by taking the solution at a series of points on a circle surrounding the scatterer.

If $p_{\text {scat }}$ is taken as a point measurement, in order to ensure that it is in the far-field, it is determined at distance $d$ from the scatterer where $k d>>1$. In the example below, $k d=20$. The size of the domain was based on $S M D=50$, and element size was based on $E P W=6$.

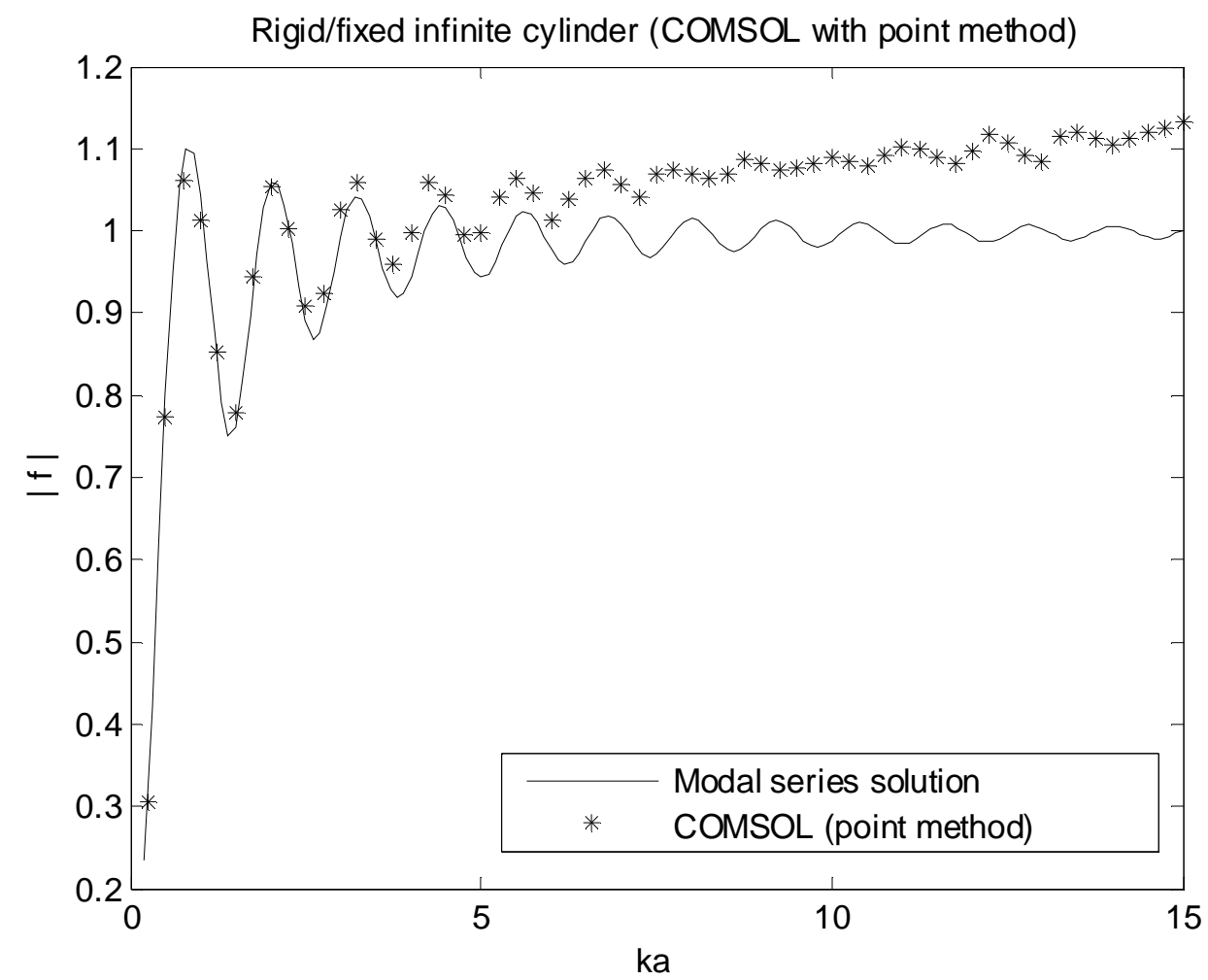

Figure 32 - Absolute value of infinite form function of a rigid/fixed infinite cylinder, using the modal series solution and COMSOL (point method). EPW $=6$ and SMD $=50$.

There are errors throughout most of the $k a$ region, with them growing with ka. 
For the circular integral method, the same values for SMD and EPW were used. The integral was performed on a circle with radius 1.1 times the cylinder radius, surrounding the scatterer.

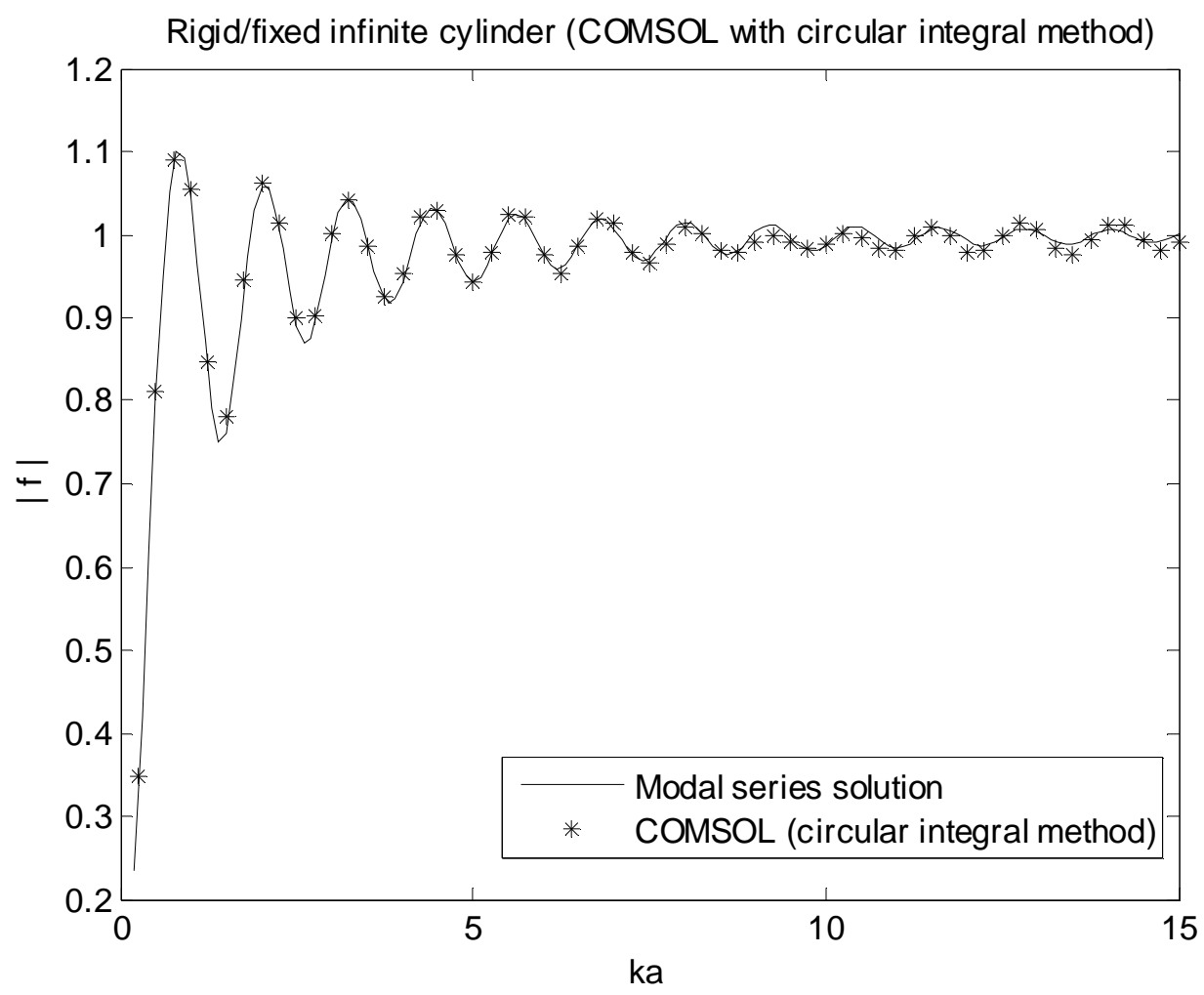

Figure 33 - Absolute value of infinite form function of a rigid/fixed infinite cylinder, using the modal series solution and COMSOL (circular integral method). EPW $=6$ and SMD $=50$.

The circular integral method succeeded in removing much of the error from the point measurement method. There is still some small error visible, caused by the discretization and radiation condition.

To determine whether or not the solution has converged with the given values of SMD and $E P W$, convergence tests were run. First, setting $S M D=50$, and varying $E P W$, the infinite form function was found for different values of $k a$ using the circular integral method. 


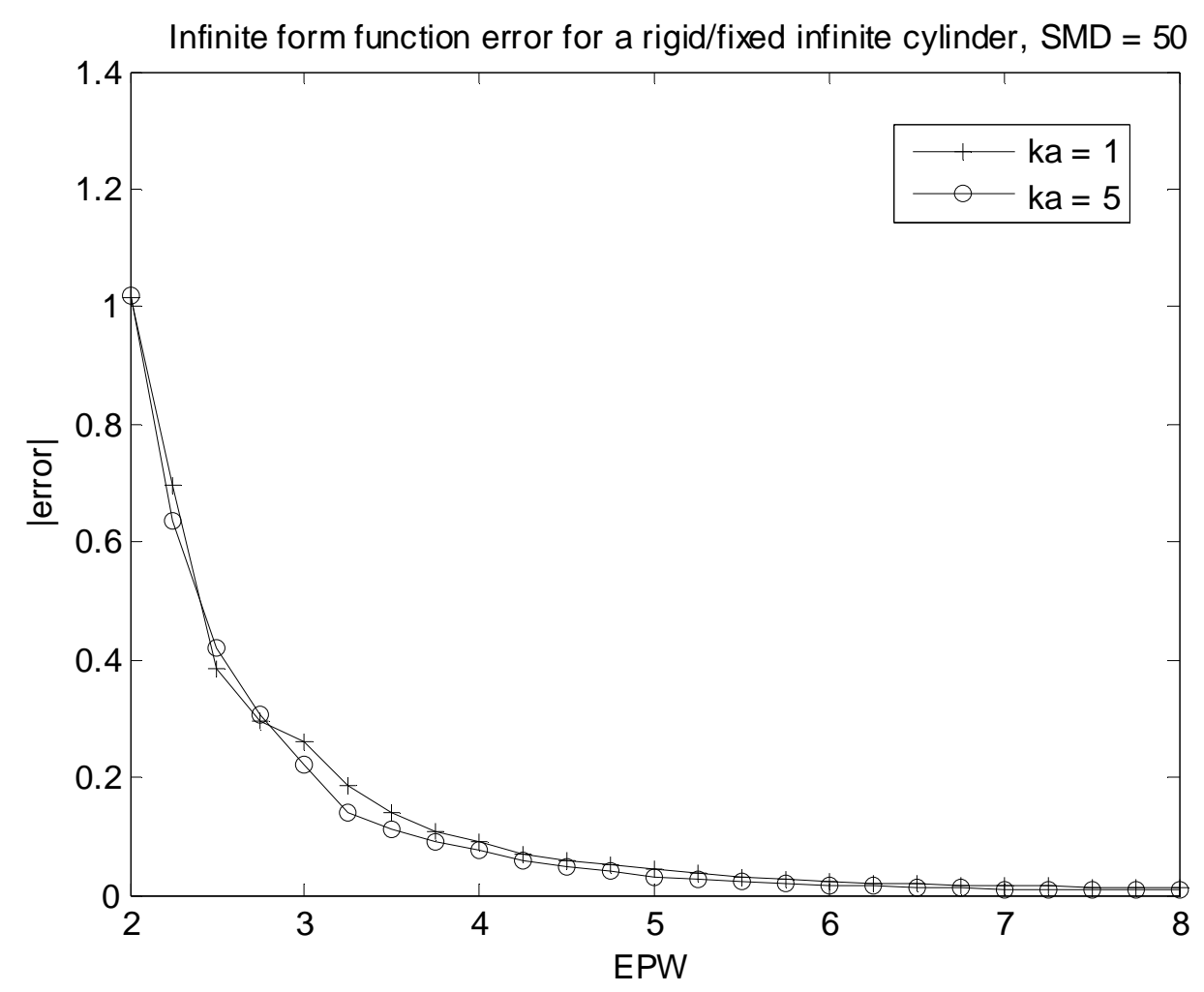

Figure 34 - Error in the infinite form function approximation of a rigid/fixed infinite cylinder at SMD $=50$.

The solution has definitely converged to a very small error at $E P W \geq 6$ for both cases. Next setting $E P W=6$, the same process was run while varying $S M D$. 


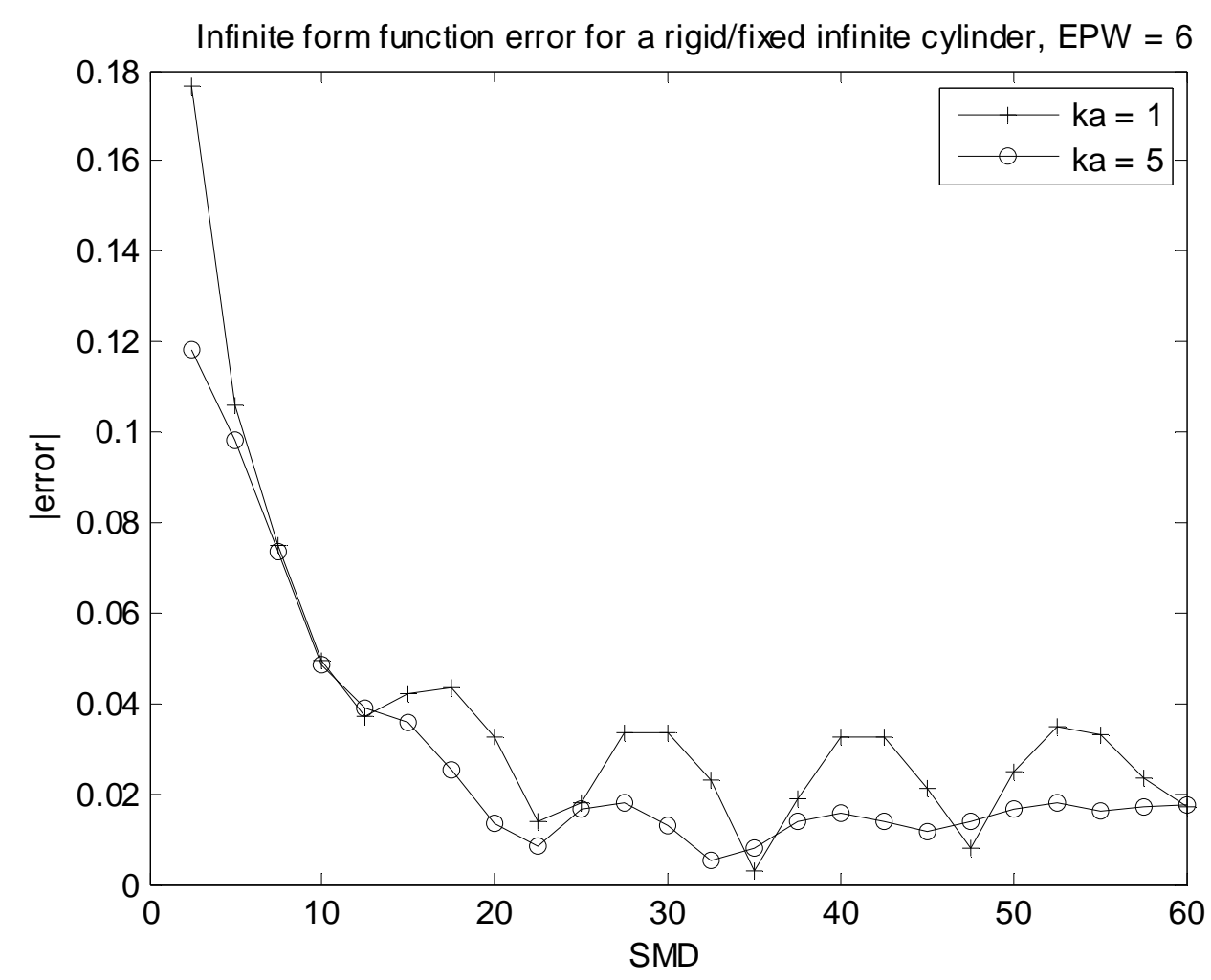

Figure 35 - Error in the infinite form function approximation of a rigid/fixed infinite cylinder at EPW $=6$.

It first should be noted that the error scale used in this figure is much smaller than that of the previous figure. Therefore, although the approximations seem to oscillate at higher values of $S M D$, the solution seems to have converged into a very small error region. From this figure it is also seen that at a certain point, increasing domain size does not seem to reduce the error effects of the radiation condition.

\subsubsection{Three Dimensional Test: Rigid/Fixed Sphere}

A three dimensional scattering problem with known solution is that of a rigid/fixed sphere (equation (2.19)). The added dimension greatly increases the number of finite elements 
for a given domain size. Often the domain of the problem cannot be extended into the far-field due to computational constraints. Therefore, the spherical integral method proves very useful in allowing near-field data to be used to calculate a value in the far-field so that target strength may be determined. Available computer resources limited three dimensional COMSOL simulations to the use of $E P W=6$ and $S M D=20$, with each simulation requiring several hours. Using these parameters, the target strength of a rigid/fixed sphere, radius $5 \mathrm{~cm}$ was calculated and compared with the exact modal series solution (section 2.3.2).

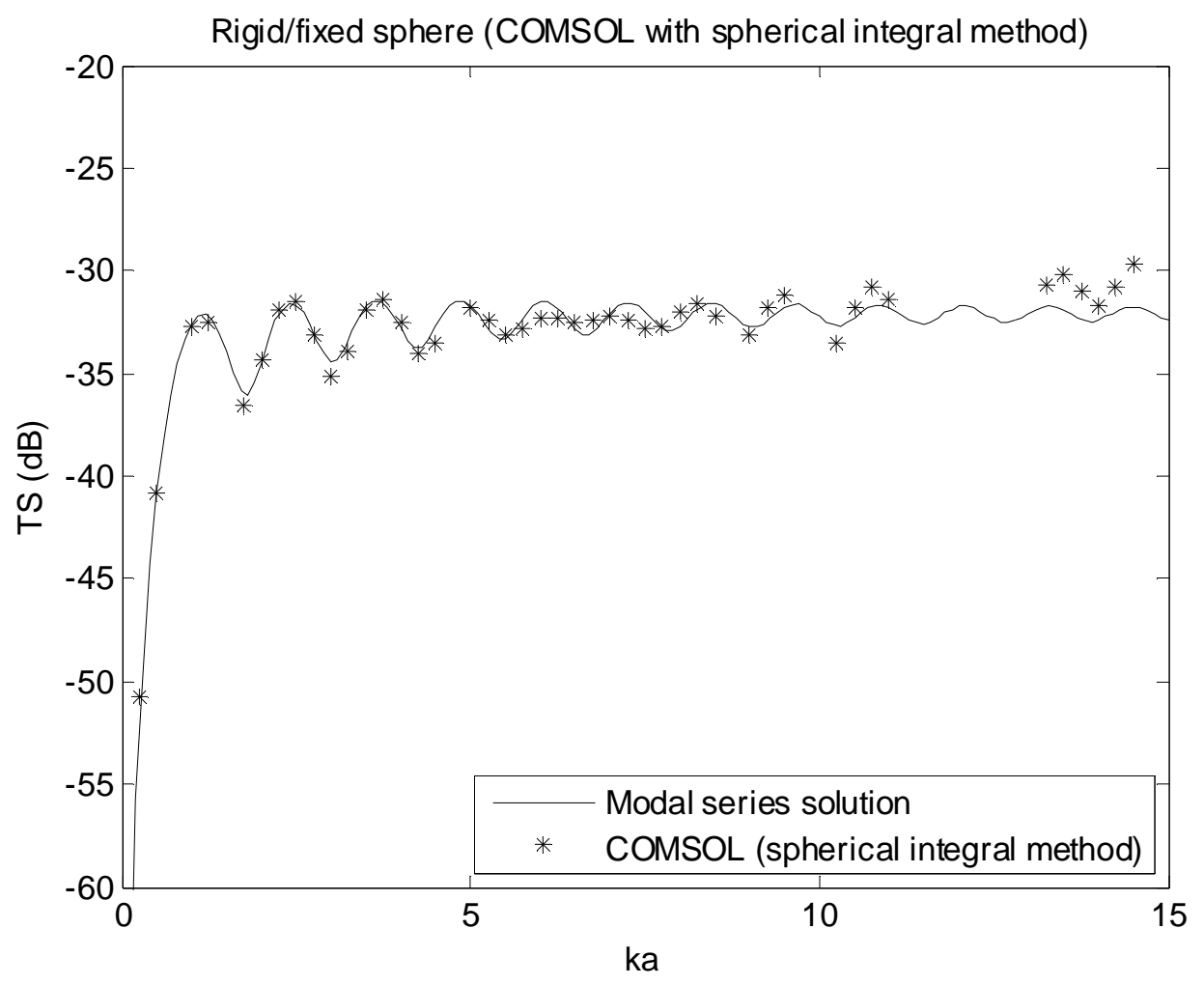

Figure 36 - Target strength of a rigid/fixed sphere, radius $5 \mathrm{~cm}$, using the modal series solution and COMSOL (spherical integral method). EPW $=6$ and SMD $=20$.

At values of ka less than five, the finite element results are very good. They are able to capture the proper peak null structure of the exact solution much better than the Kirchhoff method. This 
is because of the finite element method does not ignore the effects of diffraction. However at higher values of ka, the results begin to diverge from the exact solution, causing errors on the order of 2-3 dB. This is an effect of some error in the finite element method, most likely caused by the radiation condition used.

\subsubsection{Methodology for Sand Dollar Predictions}

The process of predicting scattering from any three dimensional object is similar to that of the sphere in the previous section. The only difference lies in the creation of the scatterer geometry, which has been discussed. For the predictions of the scattering from the sand dollar, a disk was used to simplify this geometry. As was seen with the rigid/fixed sphere, errors on the order of 2-3 dB were expected from the use of COMSOL, likely stemming from the choice of radiation condition. However, the experimental sand dollar scattering data were such that these errors were small enough for the finite element method to be useful. The results are presented and compared with experimental data in Chapter 4.

\subsection{Chapter Summary}

The finite element method has been explored in the application of acoustic scattering problems. Techniques for reducing numerical error after the solution process in two and three dimensions were presented. These techniques, referred to here as the circular and spherical integral methods, also allow scattered pressure values to be calculated at points outside of the original domain. COMSOL Multiphysics was explored in the simple tests of scattering from a rigid/fixed infinite cylinder and a sphere. The finite element method is used to predict the 
scattering from rigid/fixed disks in Chapter 4. These results are compared to the experimental data of scattering from a sand dollar. 


\section{Chapter 4 Model Results and}

\section{Comparison to Laboratory}

\section{Experimental Data}

\subsection{Introduction}

In this chapter, the sand dollar scattering models are explored, and predictions are compared to laboratory experimental results. The models consist of the analytic and numerical solutions of the Kirchhoff integral and solutions from the finite element method. Before introducing the experimental data, the model predictions are compared with one another. All model predictions are based on rigid/fixed boundary conditions. The analytic solution of the Kirchhoff integral for a disk, chosen as a simple approximation to the geometry of the flat side of the sand dollar, is compared with the predicted results from the finite element method for a disk over both ranges of frequency and orientation. Then, the model predictions for the rigid/fixed disk and spherical cap are compared to the experimental results from Stanton and Chu (2004) of the scattering from an aluminum disk and a sand dollar. These comparisons are conducted over a range of angles of orientation, -20 to 80 degrees from broadside, at a single frequency, $70 \mathrm{kHz}$. This range of angles was chosen due to symmetry and difficulties in the finite element method near 90 degrees. In addition to the analytic solutions of the Kirchhoff integral and the finite element method for a rigid/fixed disk, a numerical solution of the 
Kirchhoff integral is also compared with the experimental results. This numerical solution is found for a rigid/fixed three dimensional model of the sand dollar from the experiments, assembled from CT scans. Finally, there is significant evidence that rigid/fixed boundary conditions are not appropriate for an aluminum disk, and a heuristic approach for accounting for the penetrable boundary condition is presented. This correction is also applied to the model predictions of scattering from the sand dollar, as the boundary conditions appropriate for a sand dollar are not accurately known.

\subsection{Comparison of Predicted Scattering Based on the Kirchhoff and Finite Element Methods for a}

\section{Rigid/Fixed Disk}

In this section, the predictions from the analytic Kirchhoff method and finite element method are compared. The two models were used to calculate the target strength of a rigid/fixed disk over ranges of both frequency and angle of orientation. The analytic solution of the Kirchhoff method was derived for a rigid/fixed disk in section 2.5.2. For the finite element method solution, the program COMSOL was used. The disk was given a radius of $3.625 \mathrm{~cm}$ and a thickness of $5.5 \mathrm{~mm}$. This size was chosen based on the actual dimensions of the sand dollar from Stanton and Chu (2004), which will be seen in the following section.

The frequency dependent calculations were performed with the incident acoustic wave at broadside to the disk, allowing the finite element simulations to be run in two dimensions, resulting in a much faster and more efficient calculation with $E P W=6$ and $S M D=50$. 
Afterwards, the spherical integral method described in Chapter 3 was used to calculate target strength. The Kirchhoff and finite element methods were carried out over a range of ka, a dimensionless quantity given by the product of the wavenumber and the disk's radius, as illustrated in figure 37.

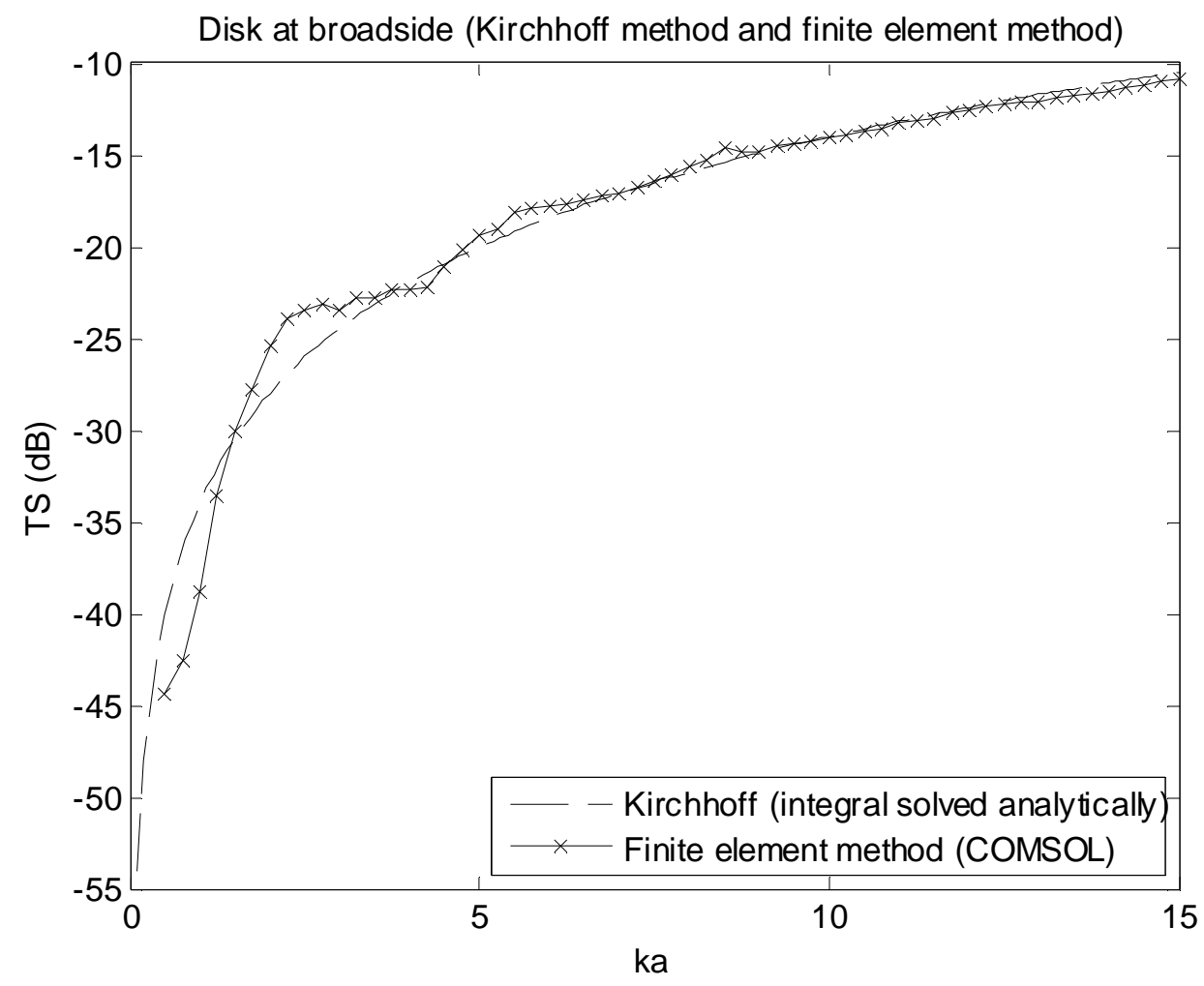

Figure 37 - Target strength at broadside incidence of a rigid/fixed disk of radius $3.625 \mathrm{~cm}$ and thickness $5.5 \mathrm{~mm}$, based on (1) the analytic solution of the Kirchhoff integral (dashed line) and (2) the finite element method using COMSOL with axial symmetry (line with $x^{\prime}$ s). EPW $=6$ and SMD $=50$.

There is very good agreement over most of the range of ka for the two models. The undulations in the finite element method predictions, particularly apparent at low ka, most likely result from diffraction effects. These are not accounted for in the Kirchhoff method, as was also seen 
when the results were compared with the modal series based solutions for the sphere and finite cylinder in Chapter 2.

The target strength of the disk was also calculated at a single frequency, $70 \mathrm{kHz}$, and over a range of angles of orientation, from zero (broadside) to eighty degrees, seen in figure 38. The disk was rotated in increments of one degree around an axis perpendicular to the incident wave vector. Because of symmetry, it only had to be rotated in one direction. The frequency of $70 \mathrm{kHz}$ was chosen to match that of the experimental data in the following section and corresponds to $\mathrm{ka}=10.6$.

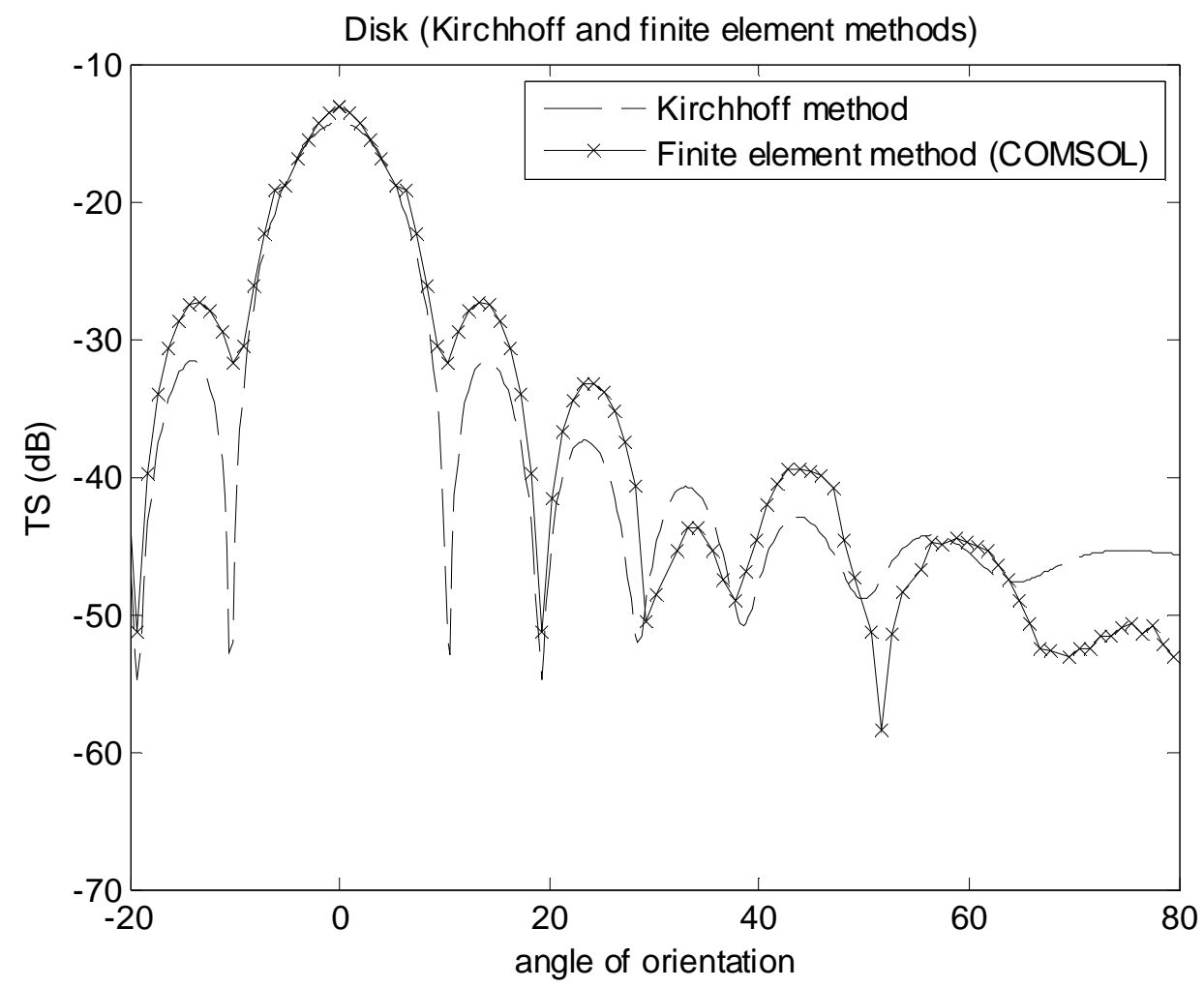

Figure 38 - Target strength at $70 \mathrm{kHz}$ of a rigid/fixed disk of radius $3.625 \mathrm{~cm}$ and thickness $5.5 \mathrm{~mm}$ based on (1) the analytic solution of the Kirchhoff integral (dashed line) and (2) the finite element method using COMSOL (line with $x^{\prime}$ ). $E P W=6$ and SMD $=20$. 
The predictions are in relatively good agreement at broadside (angle of orientation zero), expected because of the good agreement at $\mathrm{ka}=10.6$ in the frequency dependent comparison, though the finite element method predicted a slightly larger $(1.1 \mathrm{~dB})$ target strength at broadside. The side lobe structures also match up relatively well, particularly in the location of the nulls, although there are height differences of up to $5 \mathrm{~dB}$ in target strength on some of the peaks. The finite element method in three dimensions was seen in section 3.4.2.3 to have an error on the order of 2-3 $\mathrm{dB}$ in the comparisons with the modal series solution for a rigid/fixed sphere. This suggests that the differences in predicted target strength for some of the side lobes are not entirely an error but possibly an effect of diffraction. The maximum deviations in the predictions generally increase with angle of orientation, suggesting that the effects of diffraction increase in this region as well.

\subsection{Experiment Background}

There are published data of the forward scattering and backscattering from a sand dollar test and a machined aluminum disk of similar size (Stanton and Chu, 2004). Data for scattering from a bivalve were also collected in that study but are not described here. In the experiment, the targets were placed individually in a large freshwater tank and subjected to broadband chirps over the frequency range 40-95 kHz. The targets were each rotated in the horizontal plane in increments of one degree over 360 degrees around a semi-major axis perpendicular to the direction of the incident wave. The results were processed in real time to remove any multipath echoes from the walls of the tank. The target information is listed in table 1. 


\begin{tabular}{|l|c|c|c|}
\hline Target & $\begin{array}{c}\text { Horizontal dimension } \\
\mathbf{( c m )}\end{array}$ & $\begin{array}{c}\text { Vertical dimension } \\
\text { (cm) }\end{array}$ & $\begin{array}{c}\text { Upper camber } \\
\text { (cm) }\end{array}$ \\
\hline $\begin{array}{l}\text { Sand dollar (Dendraster } \\
\text { excentricus) }\end{array}$ & 7.25 & 6.7 & .1 \\
\hline $\begin{array}{l}\text { Bivalve (Dinocardium } \\
\text { robustum vanhyningi) }\end{array}$ & 7.0 & 6.9 & 2.9 \\
\hline Aluminum disk & 8.0 & 8.0 & 0.19 \\
\hline
\end{tabular}

Table 1 - Target information for the acoustic scattering experiment performed by Stanton and Chu (2004).

The upper camber measurement in table 1 refers to the height of the targets when placed on a flat surface (sand dollar on its flat side and bivalve on its concave side). The value for the aluminum disk refers to its thickness, $1.9 \mathrm{~mm}$. Computed tomography $(\mathrm{CT})$ scans were also obtained for the sand dollar at the Marine Research Facility at the Woods Hole Oceanographic Institution, illustrating well the interior of the test.

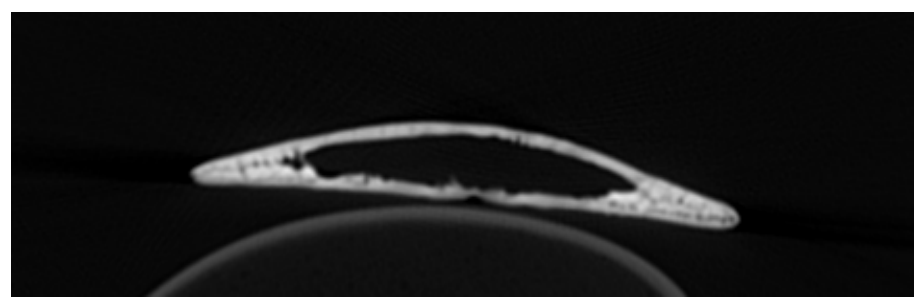

Figure 39 - Cross section of a sand dollar (Dendraster excentricus) obtained from CT scans. Details of the inner structure are revealed in addition to the high-resolution measurements of the outer shape (from Andone Lavery, personal communication). 


\subsection{Comparisons of Acoustic Scattering Predictions to Experimental Data}

The predicted scattering based on the Kirchhoff and finite element methods can be compared to the experimental data obtained by Stanton and Chu (2004). Each comparison is performed over a range of angles of orientation at a frequency of $70 \mathrm{kHz}$. First the experimental results for the aluminum disk are compared to the analytic solution of the Kirchhoff integral for a rigid/fixed disk and the finite element solution for a rigid/fixed disk. Next, the experimental results for scattering from the flat side of the sand dollar are compared with the analytic solution of the Kirchhoff integral for a rigid/fixed disk, the numerical solution of the Kirchhoff integral for the flat side rigid/fixed sand dollar model (which uses the actual shape of the sand dollar obtained from CT scans), and the finite element method solution for a rigid/fixed disk. Finally, the experimental results for scattering from the round side of the sand dollar are compared to the analytic solution of the Kirchhoff integral for a rigid/fixed spherical cap and the numerical solution of the Kirchhoff integral for the round side rigid/fixed sand dollar model. Time limitations prevented the comparison of the finite element method solution for the rigid/fixed sand dollar model (either flat side or round side) or rigid/fixed spherical cap with experimental data. Tables 2, 3, and 4 summarize the comparisons and there location within this work. 


\section{Aluminum Disk}

\begin{tabular}{|l|l|l|l|l|}
\hline & $\begin{array}{l}\text { Kirchhoff, } \\
\text { analytic }\end{array}$ & $\begin{array}{l}\text { Kirchhoff, } \\
\text { numerical }\end{array}$ & $\begin{array}{l}\text { Finite element } \\
\text { method }\end{array}$ & $\begin{array}{l}\text { Experimental } \\
\text { Data }\end{array}$ \\
\hline $\begin{array}{l}\text { Kirchhoff, } \\
\text { analytic }\end{array}$ & NA & Section $2.7 .3^{1}$ & Section $4.2^{1}$ & Section $4.4 .1^{*}$ \\
\hline $\begin{array}{l}\text { Kirchhoff, } \\
\text { numerical }\end{array}$ & Section $2.7 .3^{1}$ & NA & None $^{2}$ & None $^{2}$ \\
\hline $\begin{array}{l}\text { Finite element } \\
\text { method }\end{array}$ & Section $4.2^{1}$ & None & NA & Section $4.4 .1^{*}$ \\
\hline $\begin{array}{l}\text { Experimental } \\
\text { Data }\end{array}$ & Section $4.4 .1^{*}$ & None & NA \\
\hline
\end{tabular}

Table 2 - Comparison summary for aluminum disk. ${ }^{1}$ Comparisons were for rigid/fixed disks, but with different dimensions than the aluminum disk. ${ }^{2}$ The analytic and numerical solutions of the Kirchhoff integral for a rigid/fixed disk are identical, so to avoid redundancy, these comparisons were not made. ${ }^{*}$ These are revisited in section 4.5.2 with an added heuristic correction, known as the penetrable condition.

Sand Dollar, Flat Side

\begin{tabular}{|l|l|l|l|l|}
\hline & $\begin{array}{l}\text { Kirchhoff, } \\
\text { analytic }^{1}\end{array}$ & $\begin{array}{l}\text { Kirchhoff, } \\
\text { numerical }\end{array}$ & $\begin{array}{l}\text { Finite element } \\
\text { method }^{1}\end{array}$ & $\begin{array}{l}\text { Experimental } \\
\text { Data }\end{array}$ \\
\hline $\begin{array}{l}\text { Kirchhoff, } \\
\text { analytic }\end{array}$ & NA & Section 4.4 .2 .2 & Section 4.2 & Section 4.4 .2 .2 \\
\hline $\begin{array}{l}\text { Kirchhoff, } \\
\text { numerical }\end{array}$ & Section 4.4 .2 .2 & NA & None $^{2}$ & ${\text { Section } 4.4 .2 .2^{*}}^{*}$ \\
\hline $\begin{array}{l}\text { Finite element } \\
\text { method }\end{array}$ & Section 4.2 & None ${ }^{2}$ & NA & Section $4.4 .2 .2^{*}$ \\
\hline $\begin{array}{l}\text { Experimental } \\
\text { Data }\end{array}$ & Section 4.4 .2 .2 & Section $4.4 .2 .2^{*}$ & Section $4.4 .2 .2^{*}$ & NA \\
\hline
\end{tabular}

Table 3 - Comparison summary for sand dollar, flat side. ${ }^{1}$ Models used a rigid/fixed disk. ${ }^{2}$ This comparison is not shown due to the similarity of the analytic and numerical solutions of the Kirchhoff integral. "These are revisited in section 4.5.2 with an added heuristic correction, known as the penetrable condition. 
Sand Dollar, Round Side

\begin{tabular}{|l|l|l|l|l|}
\hline & $\begin{array}{l}\text { Kirchhoff, } \\
\text { analytic }^{1}\end{array}$ & $\begin{array}{l}\text { Kirchhoff, } \\
\text { numerical }\end{array}$ & $\begin{array}{l}\text { Finite element } \\
\text { method }^{2}\end{array}$ & $\begin{array}{l}\text { Experimental } \\
\text { Data }\end{array}$ \\
\hline $\begin{array}{l}\text { Kirchhoff, } \\
\text { analytic }\end{array}$ & NA & Section 4.4 .2 .3 & None & Section 4.4 .2 .3 \\
\hline $\begin{array}{l}\text { Kirchhoff, } \\
\text { numerical }\end{array}$ & Section 4.4 .2 .3 & NA & None & Section $4.4 .2 .3^{*}$ \\
\hline $\begin{array}{l}\text { Finite element } \\
\text { method }^{2}\end{array}$ & None & None & NA & None \\
\hline $\begin{array}{l}\text { Experimental } \\
\text { Data }\end{array}$ & Section 4.4 .2 .3 & Section $4.4 .2 .3^{*}$ & None & NA \\
\hline
\end{tabular}

Table 4 - Comparison summary for sand dollar, round side. ${ }^{1}$ Model used a rigid/fixed spherical cap, and analytic integral is solved numerically. ${ }^{2}$ Finite element method simulations for a spherical cap were not run due to insufficient resources. ${ }^{*}$ This is revisited in section 4.5.2 with an added heuristic correction, known as the penetrable condition.

\subsubsection{Aluminum Disk}

The aluminum disk experimental results are first compared with the analytic solution of the Kirchhoff integral for a rigid/fixed disk (equation (2.48)). Recall that the numerical and analytic solutions of the Kirchhoff integral for a disk are identical, and so the results for the numerical Kirchhoff solution are not shown here. The model disk was given the same dimensions as the aluminum disk from the experiment, namely a radius of $4 \mathrm{~cm}$ and a thickness of $1.9 \mathrm{~mm}$. Figure 40 shows the Kirchhoff solution and the experimental results for the aluminum disk plotted over a range of angles of orientation. 


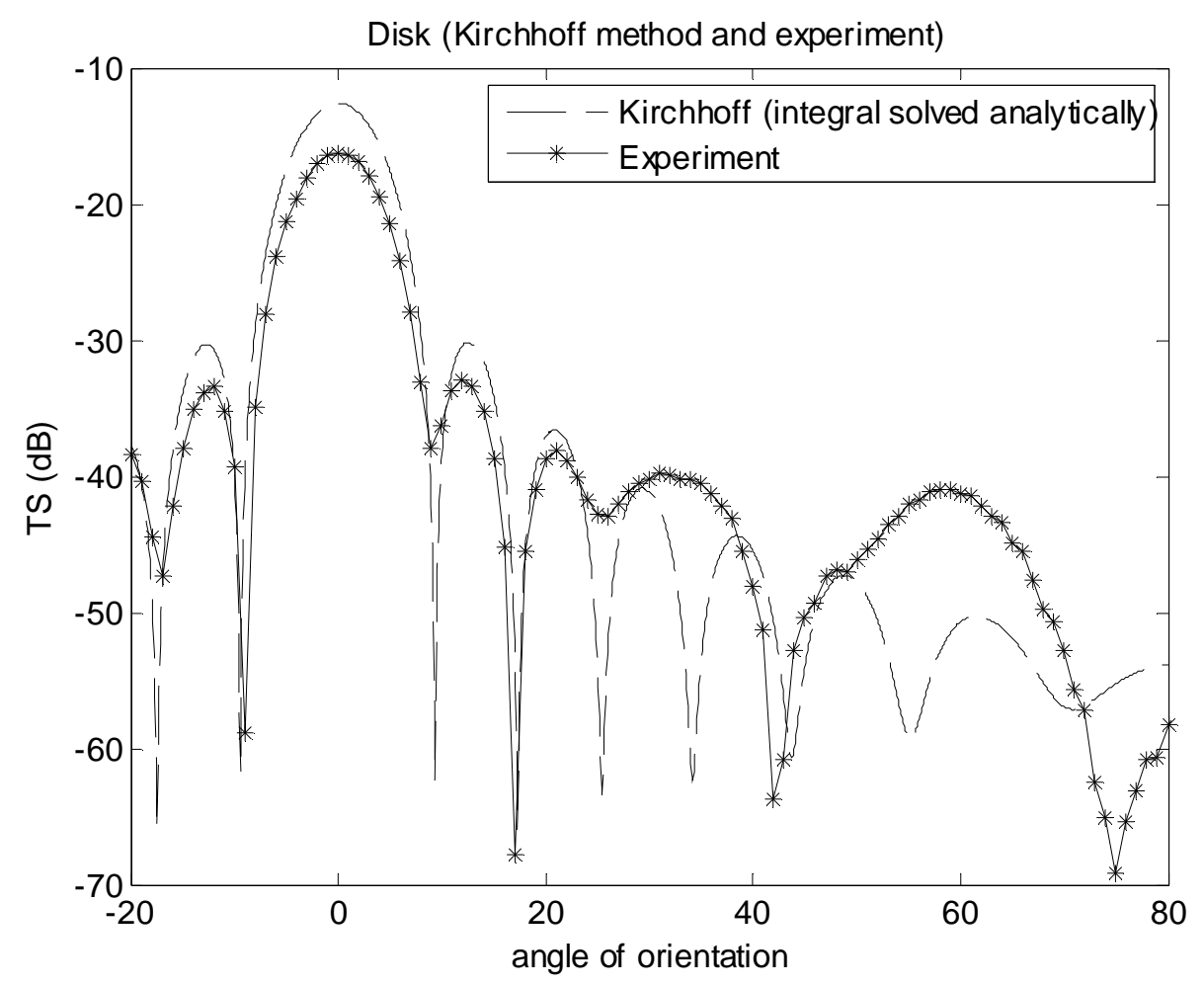

Figure 40 - Comparison of measured target strength at $70 \mathrm{kHz}$ as a function of angle of orientation for an aluminum disk (solid starred line), radius $4 \mathrm{~cm}$ and thickness $1.9 \mathrm{~mm}$, to predicted target strength of a rigid/fixed disk of similar dimensions found by solving the Kirchhoff integral analytically (dashed line).

Near broadside (approximately $+/$ - 20 degrees), there is good structural agreement between the predictions and the experimental results. The main lobe, first side lobes, and most of the second side lobes are visible in both the analytic Kirchhoff solution and the experimental results. However, the Kirchhoff integral solution predicted higher values for the peaks of these lobes, with the main peak of the Kirchhoff prediction almost $3.7 \mathrm{~dB}$ above the experimental results. The source of this error stems from either the Kirchhoff method itself or the experiment. Neglecting experimental error, the two possible sources of error in the Kirchhoff method are the neglect of diffraction and the use of a rigid/fixed boundary condition. Because of the high aspect ratio shape near broadside, the effects of elastic waves are lessened at this orientation. 
However, the rigid/fixed boundary condition still causes errors because it neglects transmission of sound into the object. In reality, not all of the incident sound wave should be reflected from the front surface of the disk as the rigid/fixed boundary condition assumes; some of it is transmitted through the front surface and is either reflected from the back of the disk or continues all the way through it. The discrepancy seems to be responsible for the Kirchhoff method predicting the higher return at broadside. At around 25 degrees away from broadside, the peak null patterns lose their similarity. The effects of elastic waves and/or diffraction are likely very important in this region, as the Kirchhoff method solution fails to match the null pattern of the experimental results.

Next, the experimental results for the aluminum disk are compared with the predictions from the finite element method for a rigid/fixed disk. Scattering simulations for a disk of radius $4 \mathrm{~cm}$ and thickness $1.9 \mathrm{~mm}$ were run in COMSOL, with $S M D=20$ and $E P W=6$. The frequency was set at $70 \mathrm{kHz}$ to match the experimental results available, and the disk was rotated in increments of two degrees over a range of angles. The far-field target strength predictions were made with the spherical integral method from Chapter 3 . Figure 41 shows the finite element method prediction and the experimental scattering results for the aluminum disk plotted over a range of angles of orientation. 


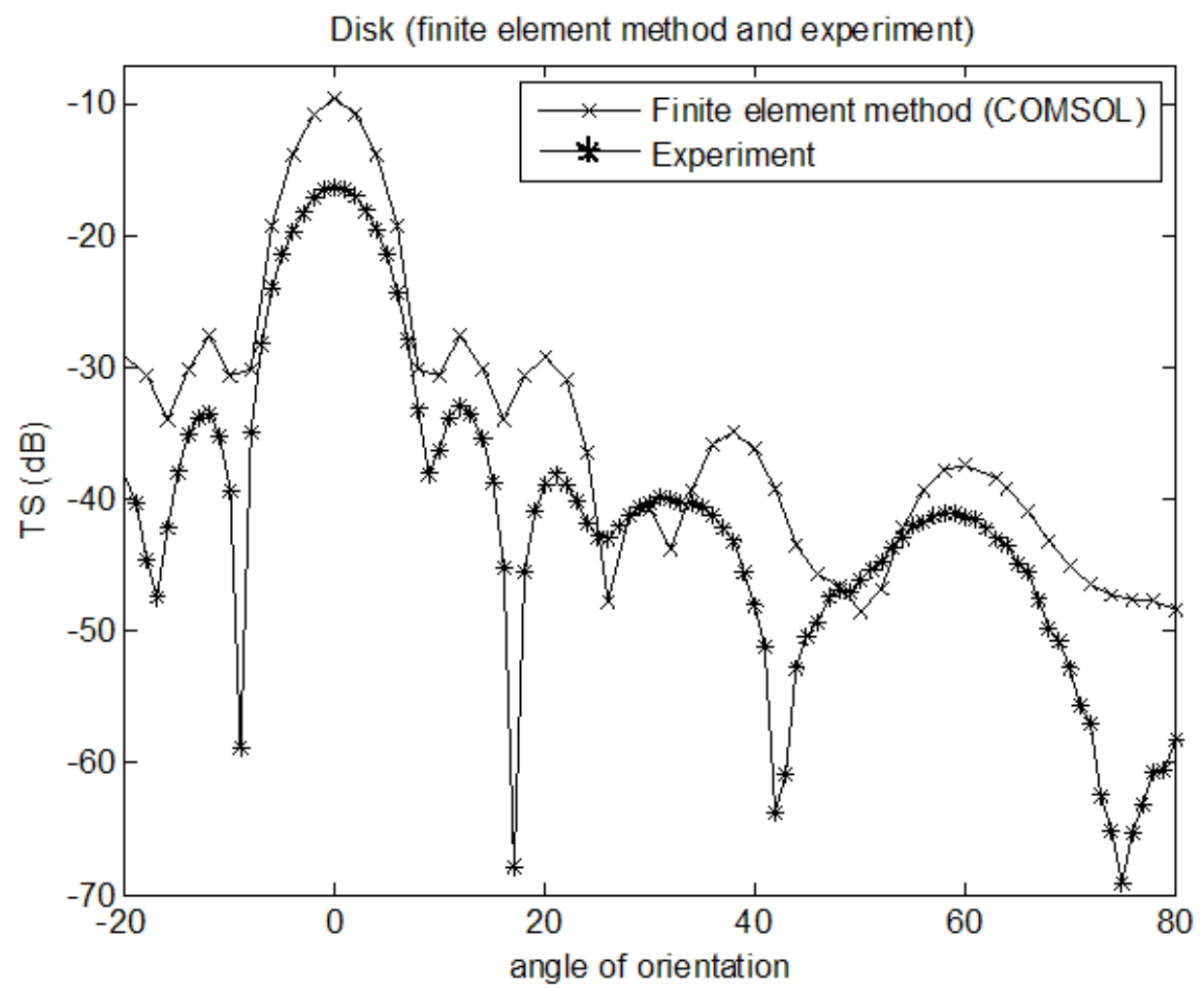

Figure 41 - Comparison of measured target strength at $70 \mathrm{kHz}$ as a function of angle of orientation for an aluminum disk (solid starred line), radius $4 \mathrm{~cm}$ and thickness $1.9 \mathrm{~mm}$, to predicted target strength of a rigid/fixed disk of similar dimensions using the finite element method in COMSOL (thin solid line with $x^{\prime}$ s). $E P W=6$ and SMD $=20$.

Like the Kirchhoff method, the finite element method predictions capture the main features of the experimental curve near broadside. Also similar to the Kirchhoff predictions, the finite element method predicts larger target strength than the experimental results in this region and is $5.1 \mathrm{~dB}$ greater at broadside. Because the finite element method incorporates the effects of diffraction, this strongly suggests that the larger returns near broadside in its prediction are due to the use of rigid/fixed boundary conditions. The finite element prediction is able to capture some of the experimental features at higher angles, particularly the peak near sixty degrees, and to a lesser extent, the peak near thirty degrees, although they are slightly shifted in angle. The 
Kirchhoff method did not accurately reproduce the experimental data in this region, suggesting that there are strong diffraction effects from the disk at higher angles of incidence.

\subsubsection{Sand Dollar}

Unlike the aluminum disk, the sand dollar does not have an analytic solution to the Kirchhoff integral. However, its complex geometry may be approximated by simpler shapes, such as a disk for the flat side and a spherical cap for the round side, which do have analytic solutions to the Kirchhoff integral (Chapter 2). Another approach, described in the next section, is to use the numerical method of solving the Kirchhoff integral by creating an accurate surface mesh of the sand dollar, obtained from CT scans. In the two sections following, the analytic solutions of the Kirchhoff integral for the simple shapes are compared with the numerical Kirchhoff solutions this new sand dollar model. This was not necessary for the disk, whose analytic and numerical solutions of the Kirchhoff integral were shown to match in section 2.7.3. Next the experimental scattering results for the sand dollar are compared with predictions from the appropriate models, described in each section. 


\subsubsection{Surface Mesh Geometry from CT Scans}

CT scans were performed on the same sand dollar that was used in the scattering experiment (Stanton and Chu, 2004) and were used to create the surface mesh necessary for solving the Kirchhoff integral numerically. Using the model reconstruction software AMIRA®, the CT scans were recreated into a three dimensional model of the sand dollar. This was imported into COMSOL and the surface mesh information was gathered for the numerical solution of the Kirchhoff integral.

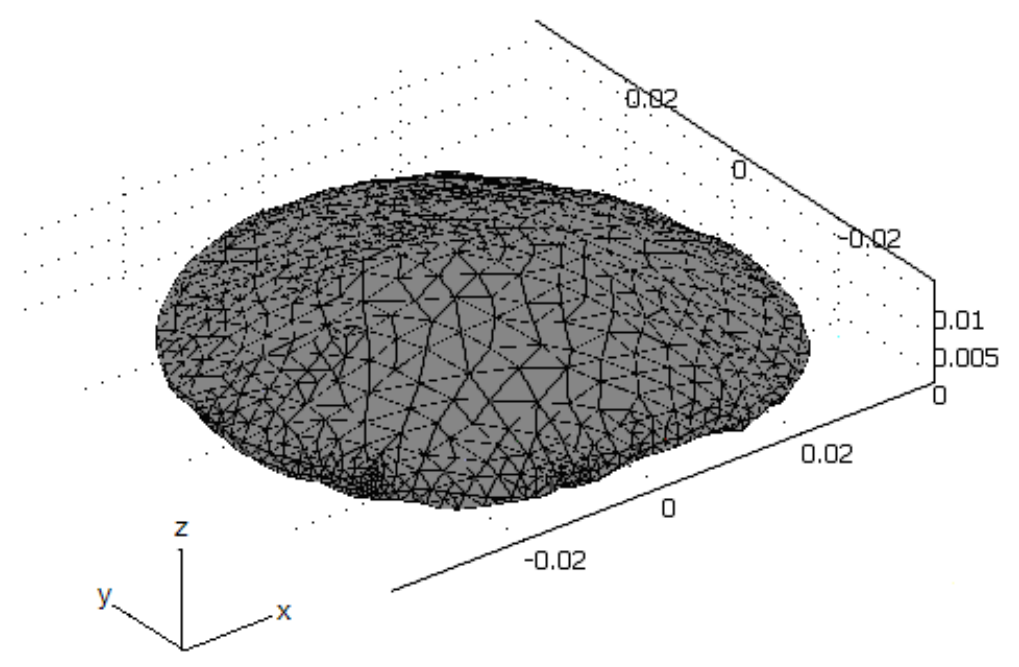

Figure 42 - Three dimensional sand dollar model in COMSOL, with the round side visible.

In the following two sections, the analytic solutions of the Kirchhoff integral for the simple shapes are first compared with the numerical solutions for the sand dollar. Then each is compared to the experimental data. This was not necessary for the disk, whose numerical and analytic solutions of the Kirchhoff integral were shown to match in section 2.7.3. 


\subsubsection{Sand Dollar Flat Side}

The experimental scattering results from the sand dollar are divided into two categories, defined by the sand dollar face oriented toward the incident wave: flat side and round side. A disk was used to model the flat side for an analytic solution to the Kirchhoff integral, presented as equation (2.48). The radius of the disk was $3.625 \mathrm{~cm}$, half of the sand dollar's horizontal dimension, and its thickness was $5.5 \mathrm{~mm}$, half of the maximum of the sand dollar's variable thickness. The choice of thickness was done to create good agreement between the analytic and numerical solutions of the Kirchhoff integral. The Kirchhoff integral was solved numerically for the surface of the sand dollar itself, using the results from the CT scans. Figure 43 shows a coarse version of the surface mesh in MATLAB, after implementing Newell's algorithm, oriented with the flat side up.

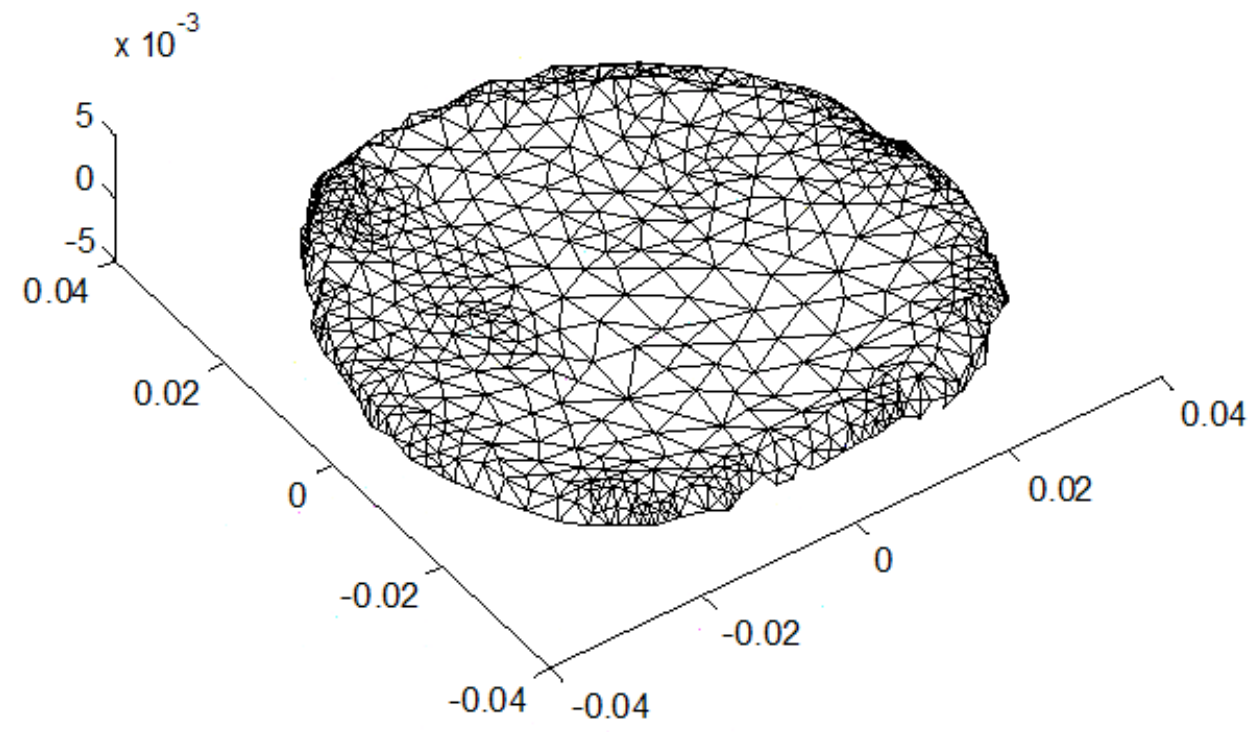

Figure 43 - Sand dollar surface mesh in MATLAB after implementing Newell's algorithm, with the orientation corresponding to an incident wave normal to the flat side. 
By comparing the two methods of solving the Kirchhoff integral, the effects of simplifying the geometry to a disk in the analytic expression can be seen. Target strength for these two models is shown in figure 44 versus angle of orientation, where zero is normal to the flat side. The axis of rotation is the y axis as seen in figure 42 .

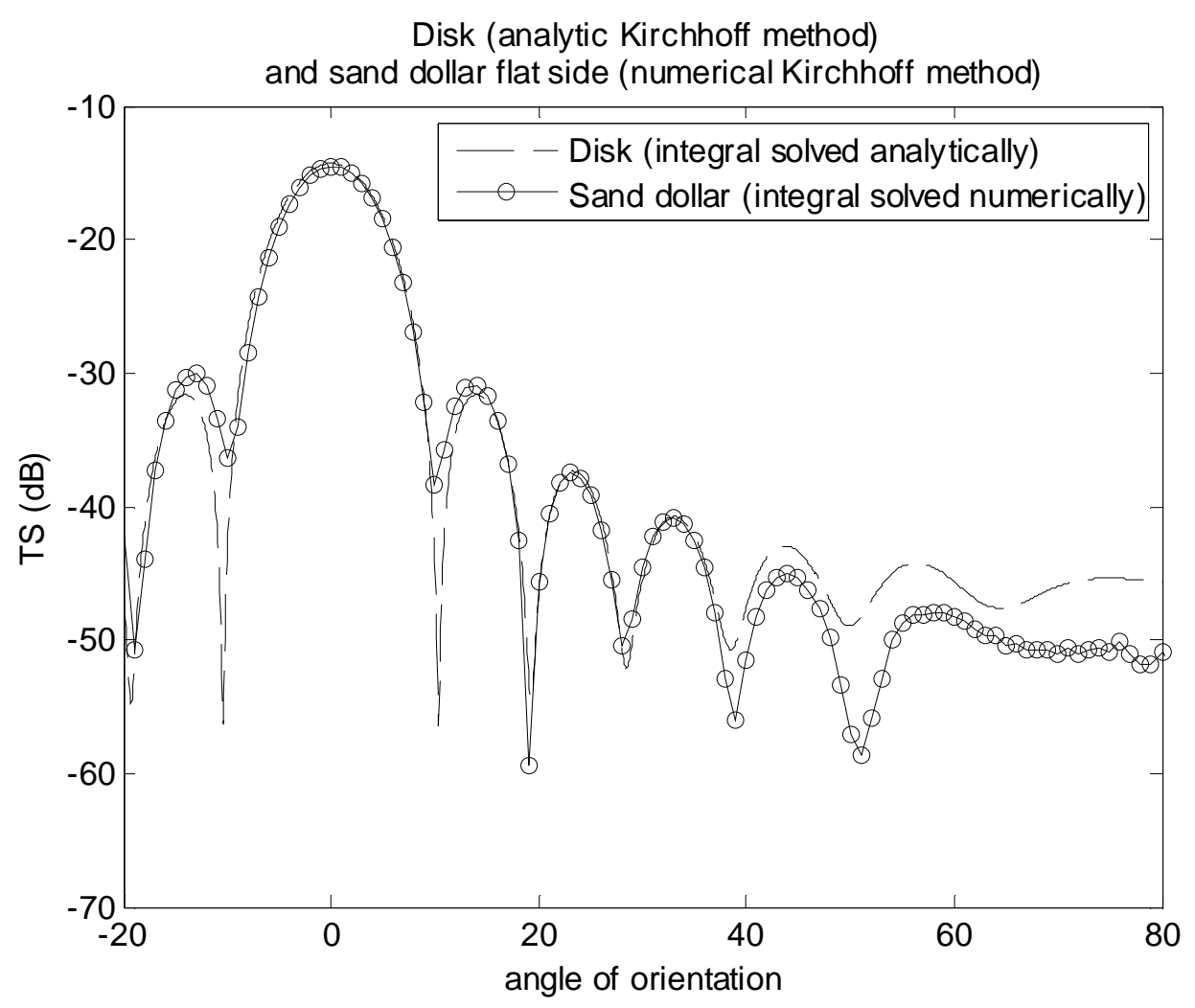

Figure 44 - Comparison of predicted target strength at $70 \mathrm{kHz}$ as a function of angle of orientation for a rigid/fixed disk, radius $3.625 \mathrm{~cm}$ and thickness $5.5 \mathrm{~mm}$, based on the analytic solution of the Kirchhoff integral (dashed line) to predicted target strength of the flat side of a rigid/fixed sand dollar of similar dimensions based on a numerical solution to the Kirchhoff integral (solid line with circles).

There is very good agreement over a wide range of angles for the two methods, with deviations starting to appear near forty degrees from broadside. These discrepancies are due simply to geometric differences between the disk and the flat side of the sand dollar. Thus, a rigid/fixed disk proves to be a good simplification of the flat side of the sand dollar when solving the 
Kirchhoff integral. The experimental data from the flat side of the sand dollar is compared with the analytic solution of the Kirchhoff integral for a rigid/fixed disk in figure 45 and the numerical solution of the Kirchhoff integral for the flat side of the sand dollar in figure 46 over a range of angles of orientation.

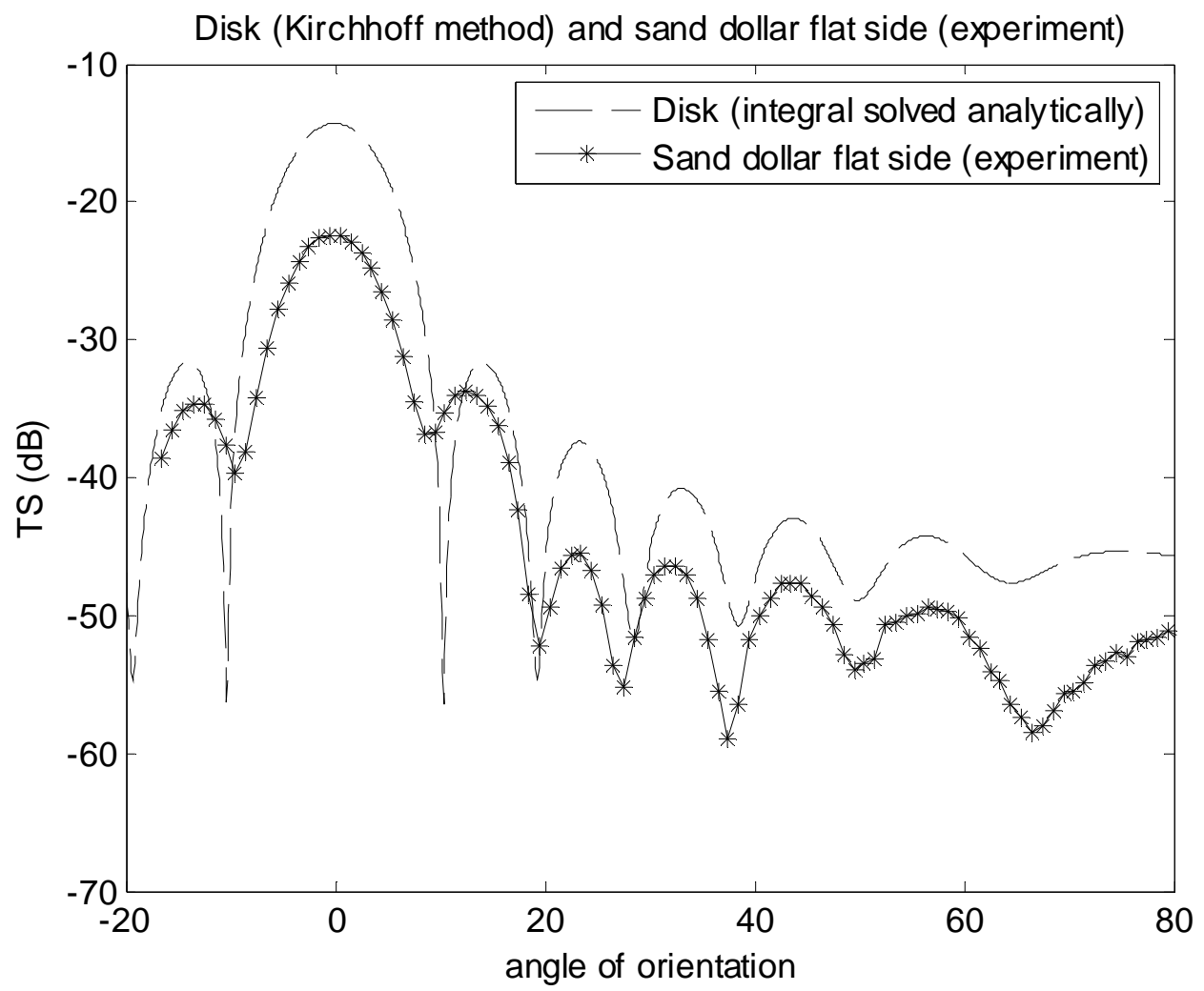

Figure 45 - Comparison of measured target strength at $70 \mathrm{kHz}$ as a function of angle of orientation for the flat side of a sand dollar (solid starred line), to predicted target strength of a rigid/fixed disk, radius $3.625 \mathrm{~cm}$ and thickness $5.5 \mathrm{~mm}$, found by solving the Kirchhoff integral analytically (dashed line). 


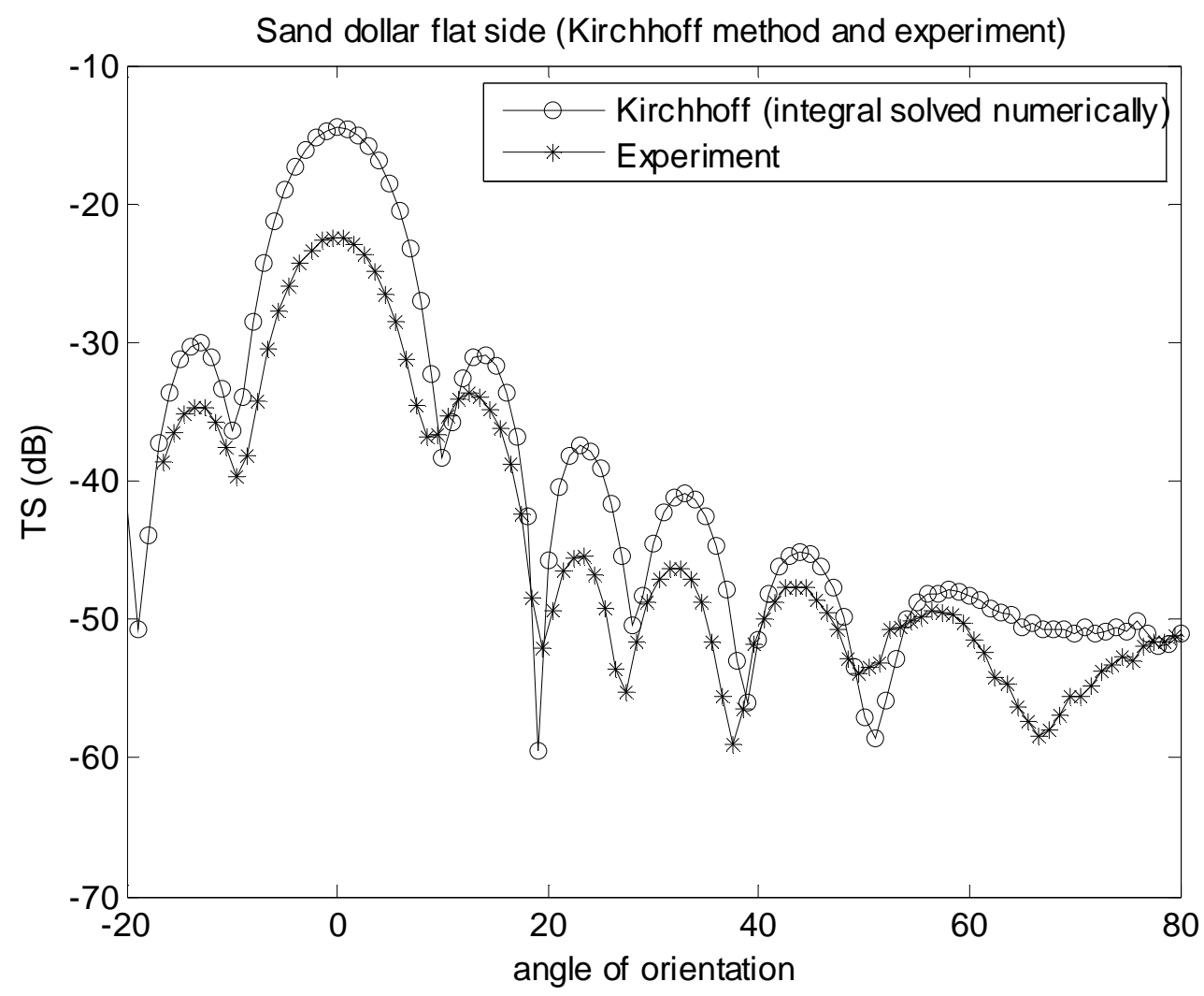

Figure 46 - Comparison of measured target strength at $70 \mathrm{kHz}$ as a function of angle of orientation for the flat side of a sand dollar (solid starred line), to predicted target strength of the flat side of a rigid/fixed sand dollar found by solving the Kirchhoff integral numerically (solid line with circles).

Because the two methods of solving the Kirchhoff integral produced such similar results, the focus will stay on figure 46 . While the lobe structures of the Kirchhoff and experimental curves are similar with respect to angle of orientation, there are significant differences between their target strength values at the locations of the peaks and nulls. Generally, the experimental data is below the predicted results from the Kirchhoff method. The main lobe of the experiment is $8.1 \mathrm{~dB}$ below the main lobe of the Kirchhoff curve. This is a similar result to the comparison with the aluminum disk. Most likely, modeling the sand dollar as a rigid/fixed scatterer is the cause of the higher predicted target strength, for the reasons mentioned before. It is important 
to note, however, the Kirchhoff solution maintains lobe structure similarity with the aluminum disk over the entire range of angles, unlike the aluminum disk.

Next, the experimental results for the flat side of the sand dollar are compared with the predictions from the finite element method, in which the flat side of the sand dollar was modeled as a rigid/fixed disk. Scattering simulations from a disk with radius $3.625 \mathrm{~cm}$ and thickness $5.5 \mathrm{~mm}$ were run in COMSOL with $S M D=20$ and $E P W=6$. The simulations were performed at a frequency of $70 \mathrm{kHz}$ and as a function of orientation, in which the disk was rotated in increments of one degree over a range of angles. The far-field calculations were obtained from the spherical integral method from Chapter 3. Figure 47 shows the finite element solution and the experimental scattering results from the sand dollar's flat side over a range of angles. 


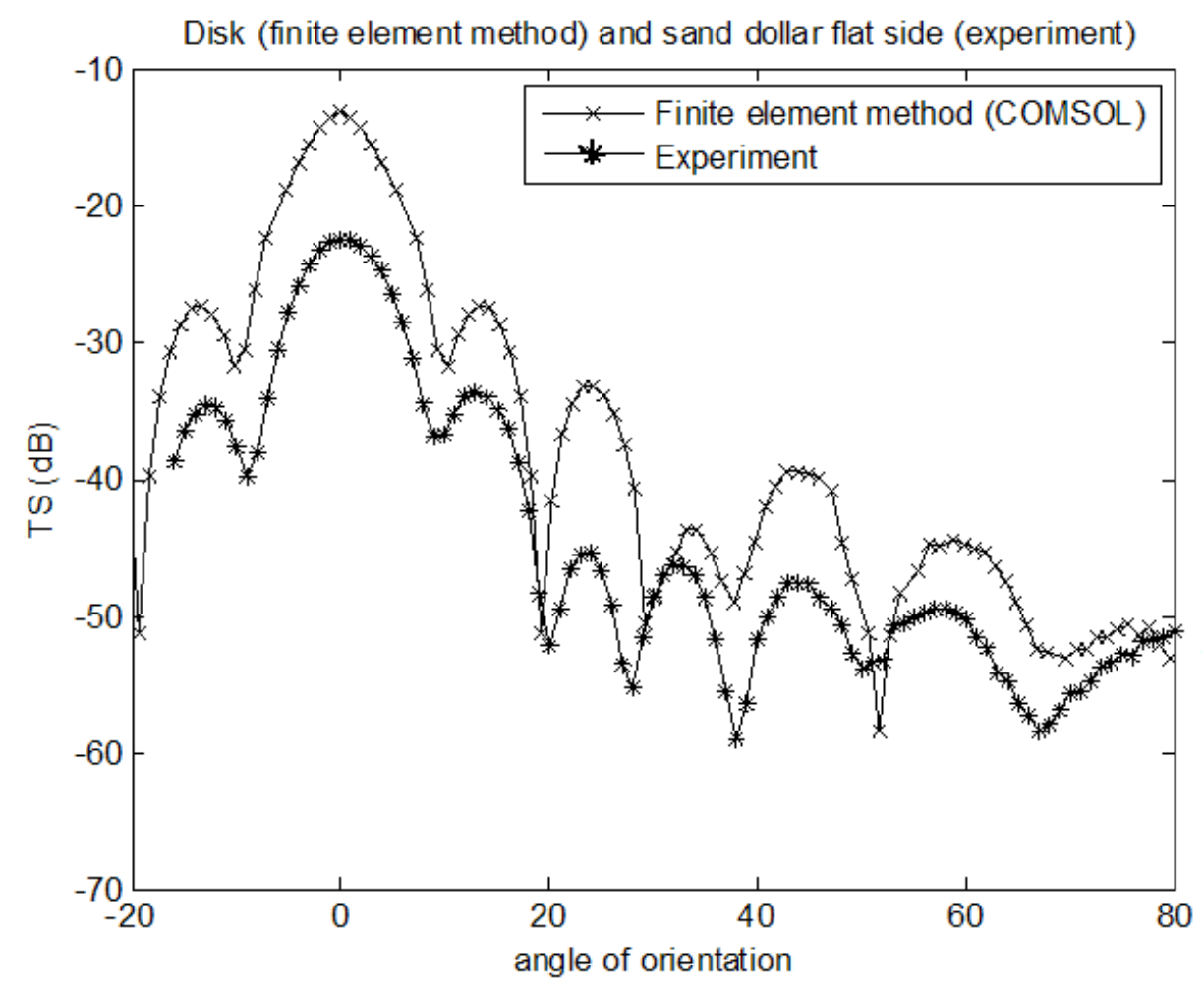

Figure 47 - Comparison of measured target strength at $70 \mathrm{kHz}$ as a function of angle of orientation for the flat side of a sand dollar (solid starred line), to predicted target strength of a rigid/fixed disk of radius $3.625 \mathrm{~cm}$ and thickness $5.5 \mathrm{~mm}$ using the finite element method in COMSOL (thin solid line with $x^{\prime}$ s). EPW $=6$ and SMD $=20$.

The predictions from the finite element method are again greater than the experimental results at low angles of incidence. At broadside, the finite element curve is about $9.2 \mathrm{~dB}$ greater than the experimental value. Again, there is not much difference in the angular dependence of the peak and nulls between the two curves.

\subsubsection{Sand Dollar Round Side}

A spherical cap was used for the solution of the Kirchhoff integral for the round side of the sand dollar. To create the cap, a sphere of radius $6.523 \mathrm{~cm}$ defined for $\theta=0$ to $\theta=33.8$ 
degrees was used. This gave a cap with radius $3.625 \mathrm{~cm}$ and thickness $1.1 \mathrm{~cm}$, the dimensions of the sand dollar. The expression for a spherical cap, equation (2.50), was evaluated with the midpoint rule in MATLAB. This process must not be confused with the numerical solution of the Kirchhoff integral using the sand dollar surface mesh. For this numerical solution, the round side of the sand dollar was modeled again using the CT scans, with a coarse mesh of the round side seen in figure 48.

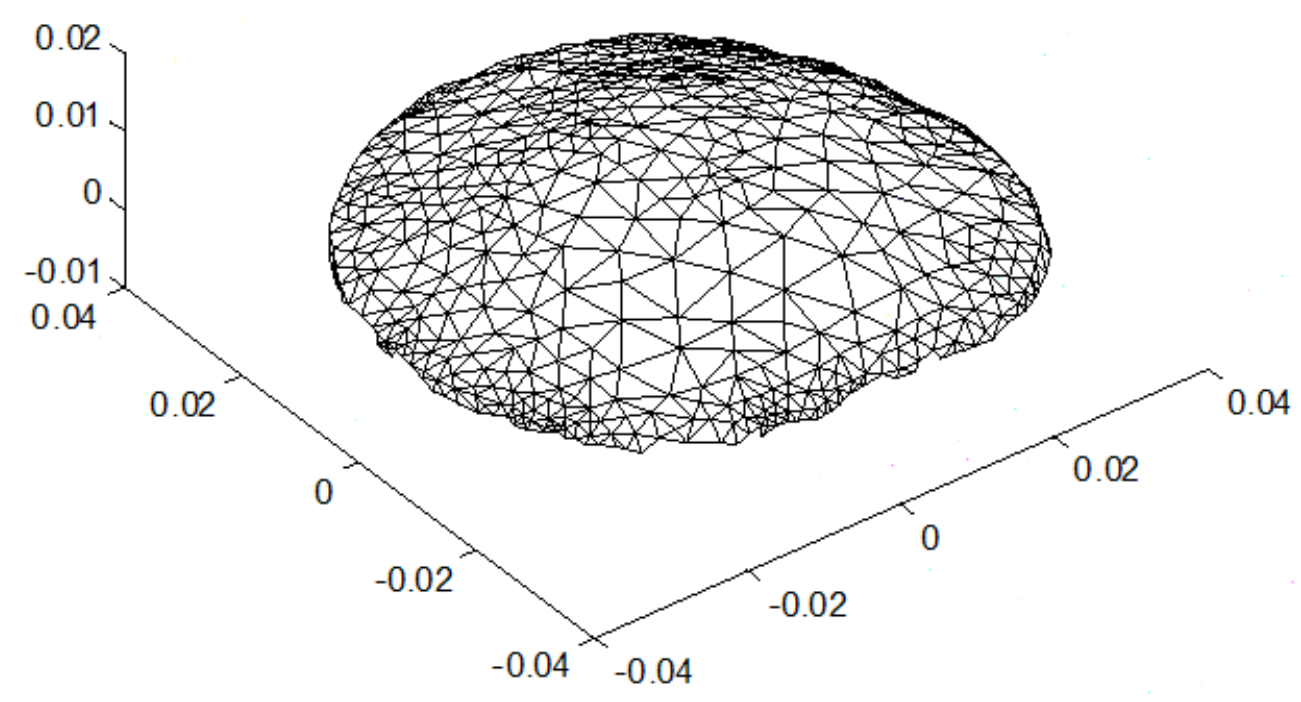

Figure 48 - Sand dollar surface mesh in MATLAB after implementing Newell's algorithm, with the orientation corresponding to an incident wave normal to the round side.

Again, these two methods of solving the Kirchhoff integral were compared to see the validity of the geometry simplification. The target strengths of the two solutions are shown in figure 49 over a range of angles of orientation, where zero corresponds to broadside. The sand dollar model was again rotated around the y axis as illustrated in figure 42 . 


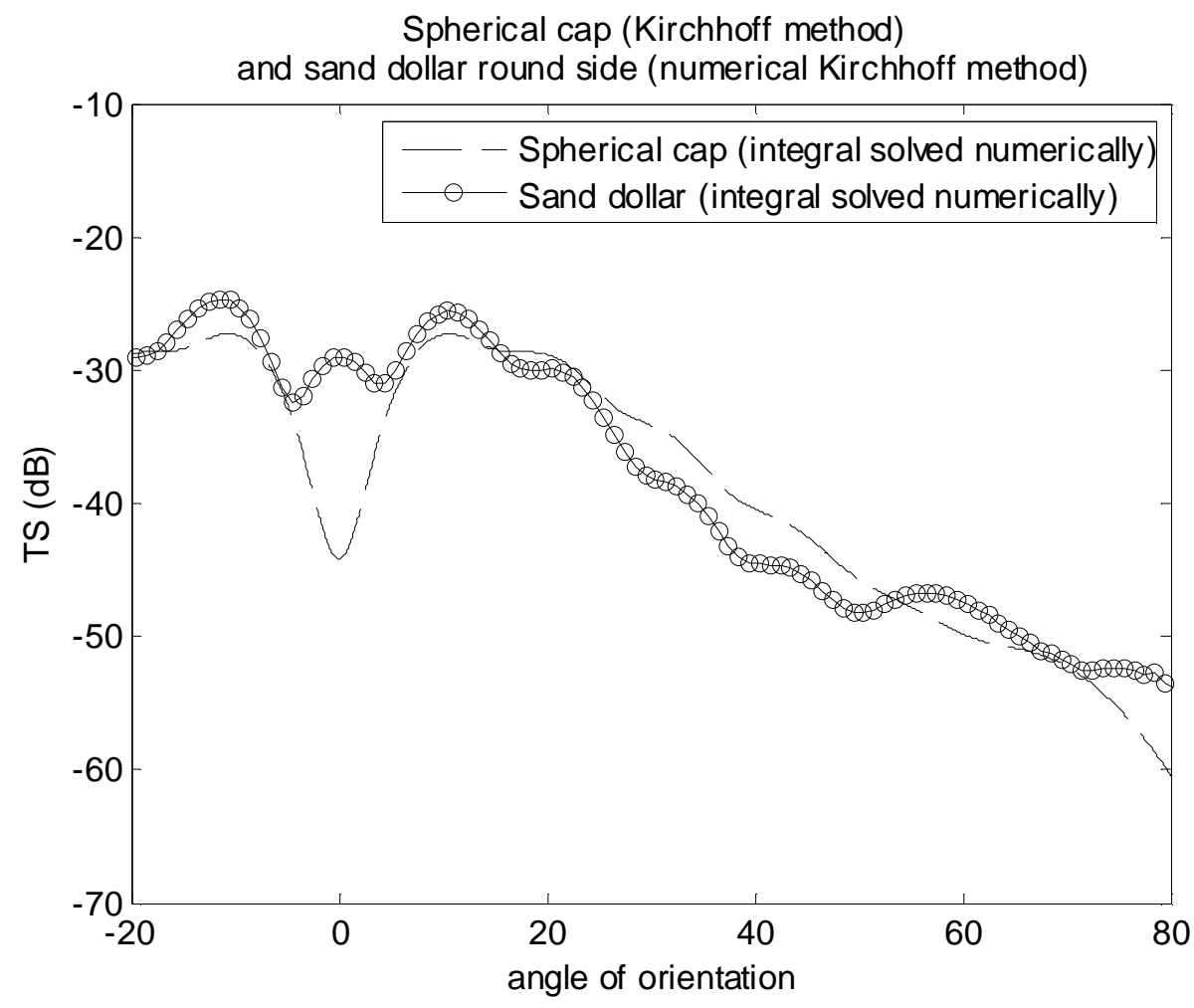

Figure 49 - Comparison of predicted target strength at $70 \mathrm{kHz}$ as a function of angle of orientation for an spherical cap, radius $3.625 \mathrm{~cm}$ and thickness $1.1 \mathrm{~cm}$, based on the analytic solution of the Kirchhoff integral (dashed line) to predicted target strength of the round side of a rigid/fixed sand dollar of similar dimensions based on a numerical solution to the Kirchhoff integral (solid line with circles).

The two predictions have some general similarities but also some very distinct differences. The agreement between the two methods is worse than that between the sand dollar's flat side and the disk. The discrepancies are most noticeable at angles close to broadside, where the spherical cap approach predicted a null, but the numerical approach predicted a peak. Interestingly, there is far less structure at angles far from broadside for the models of the sand dollar's round side as opposed to those for its flat side. However, at angles larger than approximately 15 degrees, the agreement between these two models was relatively good, with the target strength decreasing with angle and the levels in relatively good agreement. 
The experimental scattering data from the sand dollar's round side are compared with the analytic solution of the Kirchhoff method for the spherical cap in figure 50 and the numerical solution for the sand dollar model in figure 51.

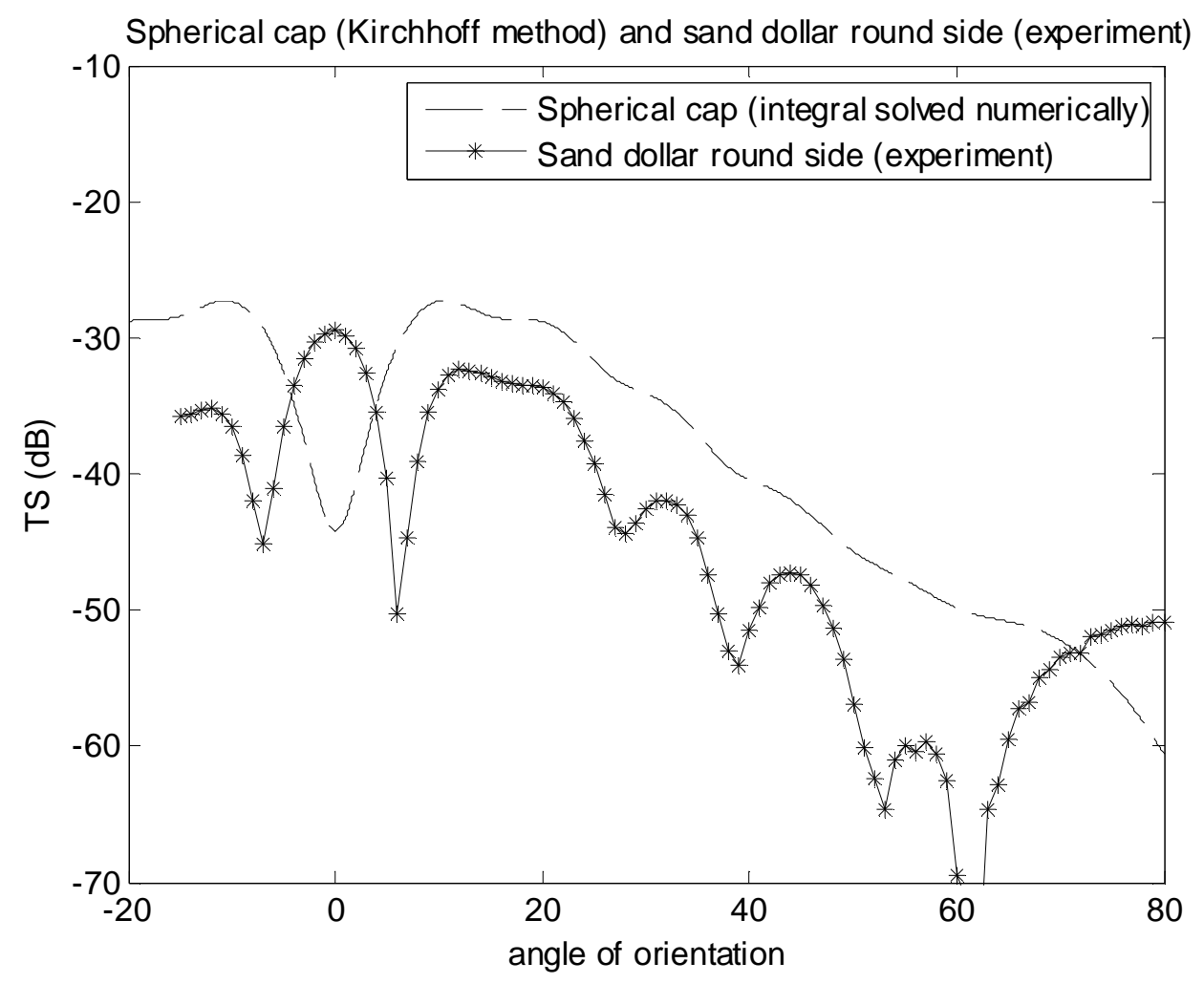

Figure 50 - Comparison of measured target strength at $70 \mathrm{kHz}$ as a function of angle of orientation for the round side of a sand dollar (solid starred line), to predicted target strength of a rigid/fixed spherical cap, radius $3.625 \mathrm{~cm}$ and thickness $1.1 \mathrm{~cm}$, found by solving the Kirchhoff integral analytically (dashed line). 


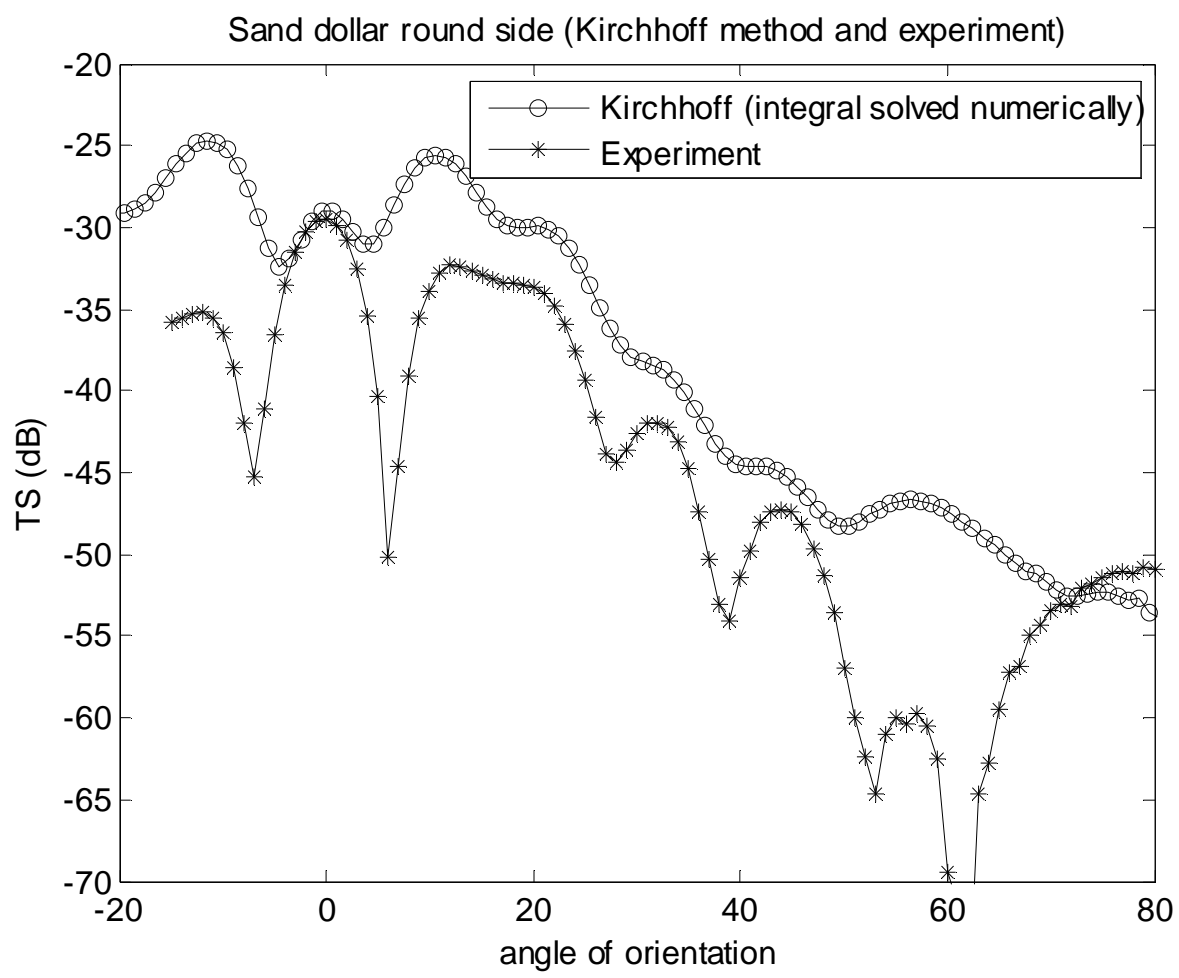

Figure 51 - Comparison of measured target strength at $70 \mathrm{kHz}$ as a function of angle of orientation for the round side of a sand dollar (solid starred line), to predicted target strength of the round side of a rigid/fixed sand dollar found by solving the Kirchhoff integral numerically (solid line with circles).

Again, the predictions based on the analytic (figure 50) and numerical (figure 51) solutions of the Kirchhoff integral mimic the experimental results in their general structure but have noticeable and important differences. The central peak at broadside incidence observed in the experimental data supports the hypothesis that the numerical Kirchhoff solution is a better model than the spherical cap. Once again, the Kirchhoff results have higher values than the experimental data, likely caused by the choice of rigid/fixed boundary conditions. While it is probable that some fraction of the incident wave is transmitted through the sand dollar in the actual experiment, this is not accounted for with the rigid/fixed boundary condition. The increased structure in the lobes of the experiment is likely caused by diffraction effects. 
Unfortunately, due to time constraints and long numerical run times, the finite element method was not tested for a spherical cap, making this hypothesis difficult to verify.

\subsection{Heuristic Improvement to Scattering Models}

\section{Developed for Rigid/Fixed Objects}

There are errors inherent in modeling an elastic scatterer as a rigid/fixed object because the portion of the wave that is transmitted through the front surface of the scatterer is neglected. In addition to neglecting the transmitted portion of the incident wave, the models presented in this thesis for aluminum disks and sand dollars have neglected the effects of elastic shear waves. The effects of elastic shear waves are extremely complicated for irregular shapes such as the sand dollar and are beyond the scope of this work. Instead, the focus of the next section will be on attempting to quantify how much of the incident wave is transmitted through versus reflected from the sand dollar. This proves easiest when the high aspect ratio shape is near broadside. As will be shown, a heuristic correction to the rigid/fixed boundary condition used for modeling the aluminum disk and sand dollar can be made that works very well near broadside incidence.

\subsubsection{Reflection Coefficients from Infinite Half-Spaces and Layers}

When a wave arrives at a boundary, some of it will be reflected and some will be transmitted into the new medium. If the boundary is planar, the reflection coefficient can quantify how much of the incident wave is reflected. If an incident wave has amplitude $p_{0}$, the 
reflected wave has amplitude $R p_{0}$. In the case of a rigid/fixed boundary, the entire wave is reflected, none is transmitted, and $R=1$. A simple case for determining $R$ is from the planar boundary of two fluid infinite half spaces. When a plane wave traveling through medium 0 $\left(c_{0}, \rho_{0}\right)$ arrives at angle $\theta$ to the normal of the boundary with medium $1\left(c_{1}, \rho_{1}\right)$, the reflection coefficient is defined as

$$
R=\frac{m \cos \theta-\sqrt{n^{2}-\sin ^{2} \theta}}{m \cos \theta+\sqrt{n^{2}-\sin ^{2} \theta}}
$$

where $n=c_{0} / c_{1}$ and $m=\rho_{1} / \rho_{0}$ (Frisk, 1994). At normal incidence, this becomes

$$
R=\frac{m-n}{m+n}
$$

Now consider the reflection off of a fluid layer in between two fluid infinite half spaces at normal incidence. A wave travels through medium $0\left(c_{0}, \rho_{0}\right)$ and arrives perpendicular to the boundary with medium $1\left(c_{1}, \rho_{1}\right)$. Medium 1 forms a layer of thickness $h$, beyond which is medium $2\left(c_{2}, \rho_{2}\right)$.

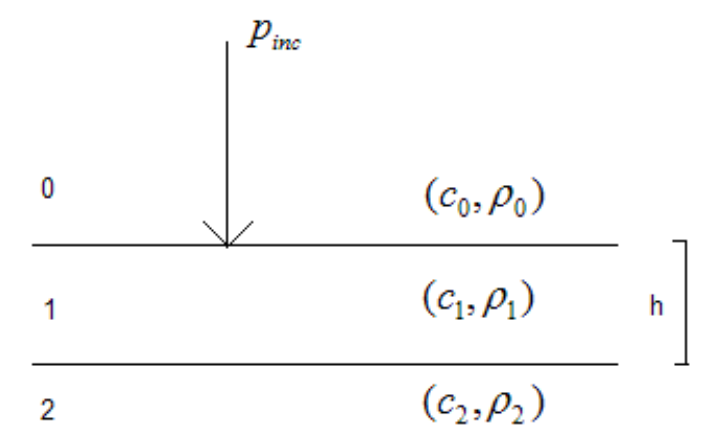

Figure 52 - Geometry of a single layer in between two infinite half spaces.

There are an infinite number of contributions to the total reflection from this layer: the wave that reflects from the front of the layer and waves that reflect within the layer any number of 
times before transmitting back into medium 0. Brekhovskikh and Lysanov (2003) give a closed form expression for the layer's reflection coefficient,

$$
R_{L}=\frac{R_{01}+R_{12} e^{2 i k_{1} h}}{1+R_{01} R_{12} e^{2 i k_{1} h}}
$$

where $R_{a b}$ is the reflection coefficient of a wave in medium $a$ off of medium $b$. The wavenumber within the layer is $k_{1}$. A similar calculation can be done if the fluid layer is replaced by an infinite elastic plate. If the incident wave arrives normal to the plate, then there is no shear stress and only compressional waves arise. Then the elastic material may be treated as a fluid.

Now imagine an incident plane wave arriving normal to $n$ layers. The $j$ th layer has thickness $h_{j}$ and material properties $c_{j}$ and $\rho_{j}$. The total reflection coefficient from the series of layers can be found using a recursive process. The total reflection from layer $n$ is found first using a modified version of the expression from Brekhovskikh and Lysanov (2003),

$$
\hat{R}_{L, n}=\frac{R_{n-1, n}+R_{n, n+1} e^{2 i k_{n} h_{n}}}{1+R_{n-1, n} R_{n, n+1} e^{2 i k_{n} h_{n}}}
$$

This can then be used to find the total reflection from both layers $n$ and $n-1$,

$$
\hat{R}_{L, n-1}=\frac{R_{n-2, n-1}+\hat{R}_{L, n} e^{2 i k_{n-1} h_{n-1}}}{1+R_{n-2, n-1} \hat{R}_{L, n} e^{2 i k_{n-1} h_{n-1}}} .
$$

The process can be continued for each layer until the total reflection is calculated. As was the case with one layer, at normal incidence, this process can be used for fluid or elastic layers, or a combination of the two. 


\subsubsection{Determination of Reflection Coefficient for Aluminum Disk and Sand Dollar}

Now the scattering from a finite bounded object is heuristically modeled by assigning it a reflection coefficient found in the same manner as the reflection coefficients of fluid and elastic layers. The scattering from an object is approximated as

$$
p_{\text {scat }}=R_{L} p_{0} \frac{e^{i k r}}{r} f_{\text {impen }}(\Omega)
$$

where $R_{L}$ is its reflection coefficient and $f_{\text {impen }}$ is the scattering amplitude of the object if it had a rigid/fixed boundary. This approximation assumes the reflection coefficient is constant everywhere on the object, which is inaccurate for many shapes due to different angles of incidence. However, this assumption is reasonable at broadside for a high aspect ratio shape such as a sand dollar or disk. In the backscattering direction with this formulation,

$$
\begin{gathered}
T S=10 \log \left|R_{L} f_{\text {impen }}\right|^{2} \\
T S=10 \log \left|f_{\text {impen }}\right|^{2}+10 \log \left|R_{L}\right|^{2} .
\end{gathered}
$$

The approximation gives that the target strength of an object is equal to its rigid/fixed target strength plus a term that is a function of its reflection coefficient. The rigid/fixed target strength is what was calculated using both the Kirchhoff and finite element methods. These results can easily be manipulated to include the effects of the reflection coefficient.

Approximations for reflection coefficients of the sand dollar and aluminum disk can be made by finding them for infinite plates with similar thicknesses and material properties. This technique should work well near broadside, where the dimensions of the objects allow such an approximation. Away from broadside, this method is not expected to perform well. The 
aluminum disk can be modeled as an infinite plate of thickness $1.9 \mathrm{~mm}$ with water $(\rho=1000$ $\mathrm{kg} / \mathrm{m}^{3}, c=1500 \mathrm{~m} / \mathrm{s}$ ) on either side. The values for the compressional sound speed and density for aluminum were taken from Molz and Beamish (1996).

\begin{tabular}{|c|c|c|c|c|c|c|}
\hline Material & $\begin{array}{c}\text { Thickness } \\
\text { (mm) }\end{array}$ & $\begin{array}{c}\text { Sound speed } \\
\text { (compress.) }\end{array}$ & Density & $\mathbf{R}_{\mathrm{L}}$ & $\left|\mathbf{R}_{L}\right|$ & $10 \log \left|R_{L}\right|^{2}$ \\
\hline Aluminum & $1.9 \mathrm{~mm}$ & $5360 \mathrm{~m} / \mathrm{s}$ & $2700 \mathrm{~kg} / \mathrm{m}^{3}$ & $\begin{array}{c}0.362- \\
0.472 \mathrm{i}\end{array}$ & 0.5954 & $-4.50 \mathrm{~dB}$ \\
\hline
\end{tabular}

Table 5 - Reflection coefficient calculation for aluminum disk.

This suggests that modeling the aluminum disk as a rigid/fixed scatterer added more than $4 \mathrm{~dB}$ to its true target strength at broadside.

The sand dollar is more complicated to model because of its irregular shape. The CT scans of the sand dollar (e.g. figure 39) provide clues on how to simplify it. The top and bottom interfaces are very distinct, separately by a fluid interior. Ignoring both the curvature of the top and the outer edge regions allows the sand dollar to be modeled as two infinite plates, with fluid layer separating them. The thicknesses of these three layers must be estimated from the CT scan itself. The maximum camber of the sand dollar is $1.1 \mathrm{~cm}$, so this places an upper bound on the width of the three layers. The sand dollar's test wall thickness is approximately $1 \mathrm{~mm}$ on both the top and bottom interfaces. The sand dollar is composed of calcite, a polymorph of calcium carbonate $\left(\mathrm{CaCO}_{3}\right)$. Carmichael (1982) gives the density of calcite as $2710 \mathrm{~kg} / \mathrm{m}^{3}$ and the compressional sound speed as $6530 \mathrm{~m} / \mathrm{s}$. The reflection coefficient can be calculated over a range of both fluid and test layer thicknesses. Water $\left(\rho=1000 \mathrm{~kg} / \mathrm{m}^{3}, c=1500 \mathrm{~m} / \mathrm{s}\right)$ was used for both the fluid layer and the fluid half spaces on either side of the three layers. 


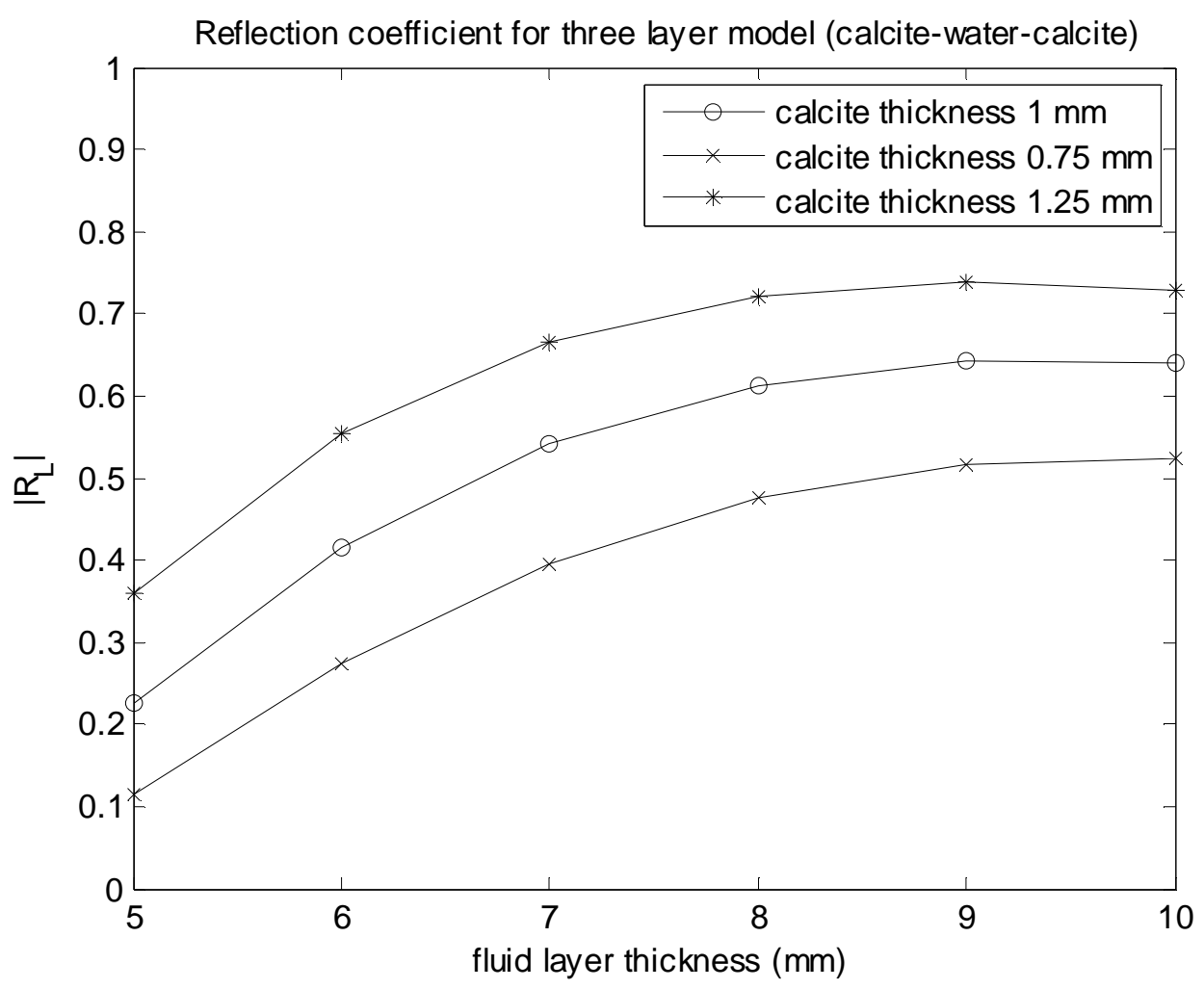

Figure 53 - Reflection coefficients from three layer model (calcite-water-calcite). Calcite thickness refers to both top and bottom layer thickness.

Because the fluid layer thickness in the actual sand dollar varies over the range plotted in figure 53 , there is a large range of reasonable reflection coefficients, approximately 0.2 to 0.7 . In order to choose the proper value for $\left|R_{L}\right|$, the rigid/fixed predicted target strengths from the models are compared to the experimental data near broadside. For the sand dollar, a reflection coefficient $\left|R_{L}\right|=0.4$ was chosen to match the Kirchhoff prediction with the experimental data for the flat side at broadside. Based on figure 53, this value is reasonable. 


\subsubsection{Heuristic Correction to the Rigid/Fixed Scattering Models}

By using the calculated reflection coefficient for an object, the heuristically corrected model values can be calculated with equation (4.7). The inclusion of the reflection coefficient into the calculations is referred to here as the penetrable condition.

\subsubsection{Aluminum Disk}

Using the value of $\left|R_{L}\right|=0.5954$ derived in the previous section for the reflection coefficient for the aluminum disk, the target strength based on both the analytic solution to the Kirchhoff integral (figure 54) and the finite element method (figure 55) are compared with the experimental data.

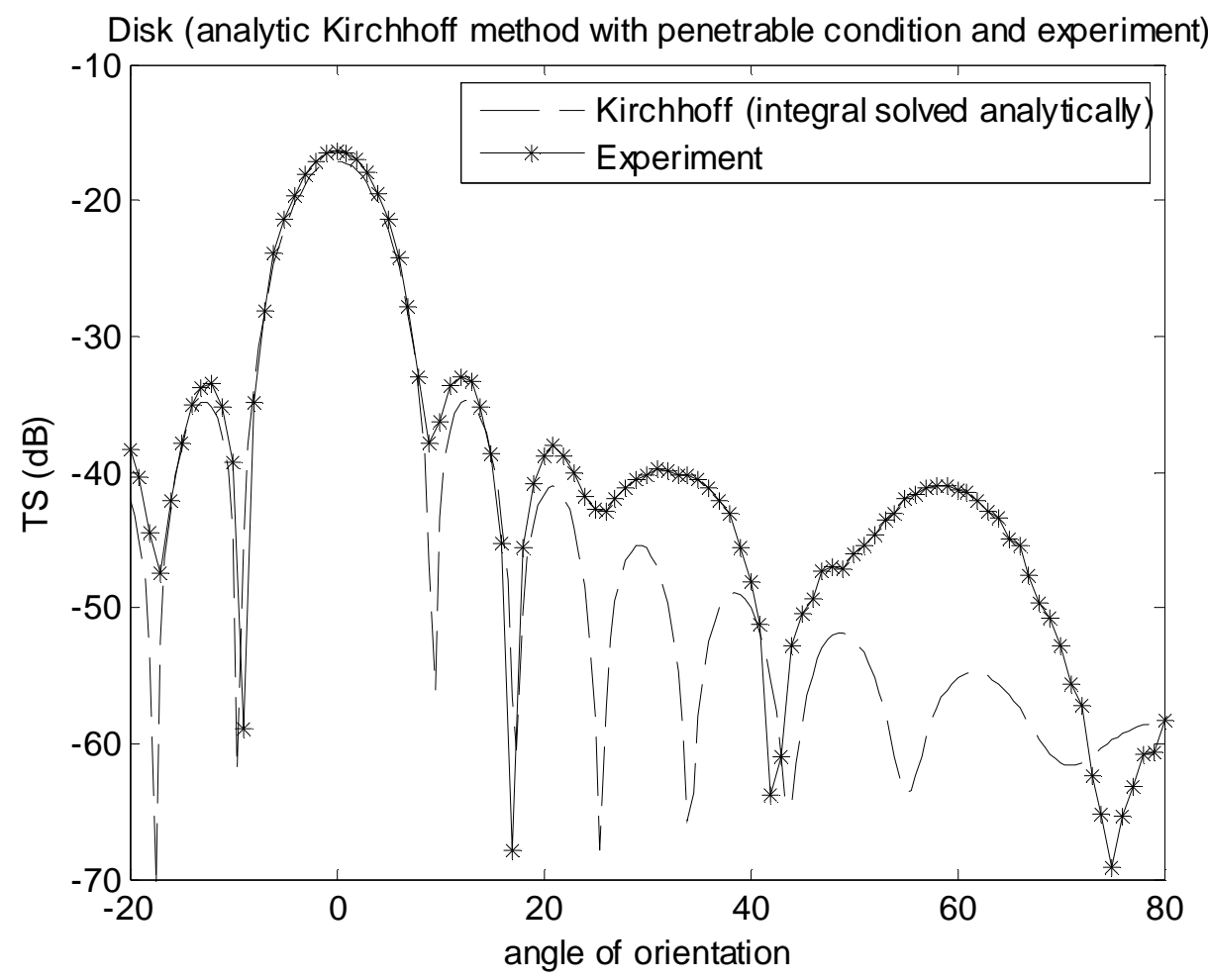

Figure 54 - Comparison of measured target strength at $70 \mathrm{kHz}$ as a function of angle of orientation for an aluminum disk (solid starred line), radius $4 \mathrm{~cm}$ and thickness $1.9 \mathrm{~mm}$, to predicted target strength of a penetrable disk $\left(\left|R_{L}\right|=0.5954\right)$ of similar dimensions found by solving the Kirchhoff integral analytically (dashed line). 


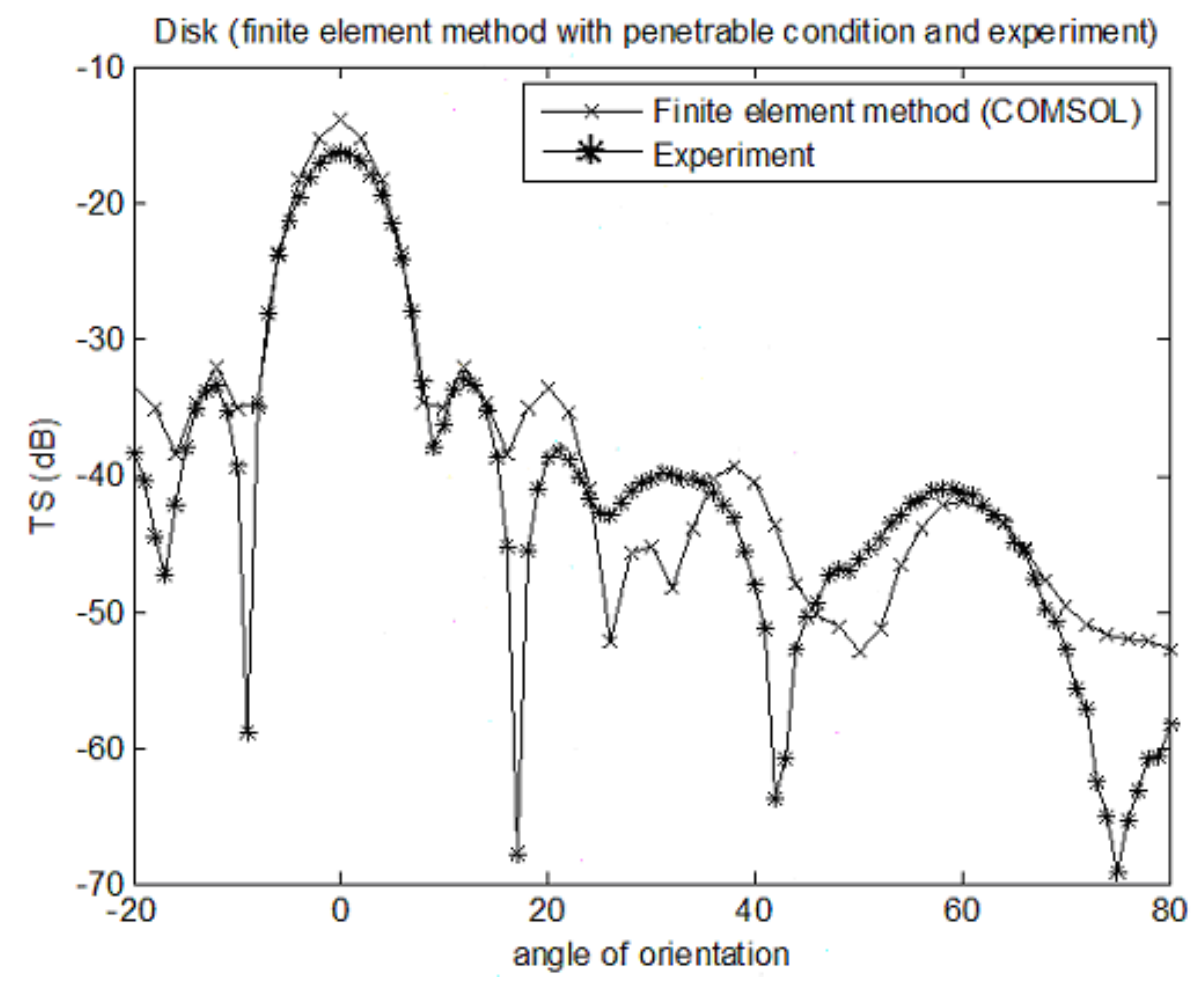

Figure 55 - Comparison of measured target strength at $70 \mathrm{kHz}$ as a function of angle of orientation for an aluminum disk (solid starred line), radius $4 \mathrm{~cm}$ and thickness $1.9 \mathrm{~mm}$, to predicted target strength of an penetrable disk $\left(\left|R_{L}\right|=0.5954\right)$ of similar dimensions using the finite element method in COMSOL (thin solid line with $x^{\prime}$ s). EPW $=6$ and SMD $=20$.

With the heuristic correction that accounts, in part, for the assumption of a rigid/fixed boundary condition, the Kirchhoff and finite element methods now predict the aluminum disk's scattering relatively well near broadside, especially along the main lobe and first pair of side lobes. The Kirchhoff prediction is within $1 \mathrm{~dB}$ of the experimental results at broadside. The finite element method is seen to have done very well away from broadside as well, capturing the large side lobe at sixty degrees. 


\subsubsection{Sand Dollar Flat Side}

The sand dollar was a more difficult shape for which to determine reflection coefficient because of its multiple interfaces and differing thickness. For the flat side, a value of $\mid R_{L}=0.4$ was chosen and was shown to be reasonable using the three layer approach. This value was chosen so the value of the numerical solution to the Kirchhoff integral at broadside would closely match that of the experimental data. Figure 56 shows the Kirchhoff method prediction for the sand dollar's flat side with the penetrable condition, compared with the experimental data for the sand dollar's flat side.

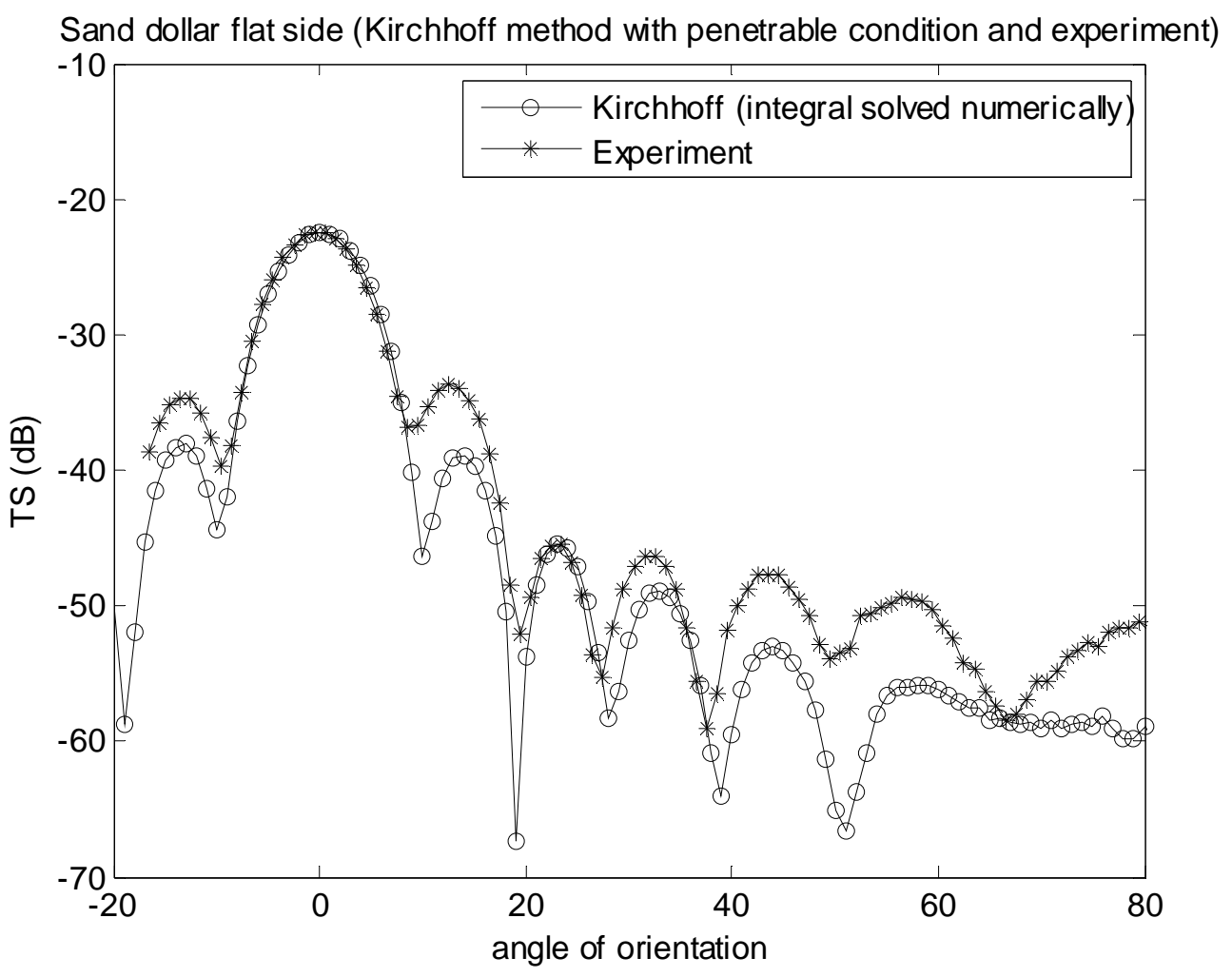

Figure 56 - Comparison of measured target strength at $70 \mathrm{kHz}$ as a function of angle of orientation for the flat side of a sand dollar (solid starred line), to predicted target strength of the flat side of penetrable sand dollar $\left(\left|R_{L}\right|=0.4\right)$ found by solving the Kirchhoff integral numerically (solid line with circles). 
Figure 57 shows the finite element method solution for a disk with the penetrable condition, compared with the experimental data for the sand dollar's flat side.

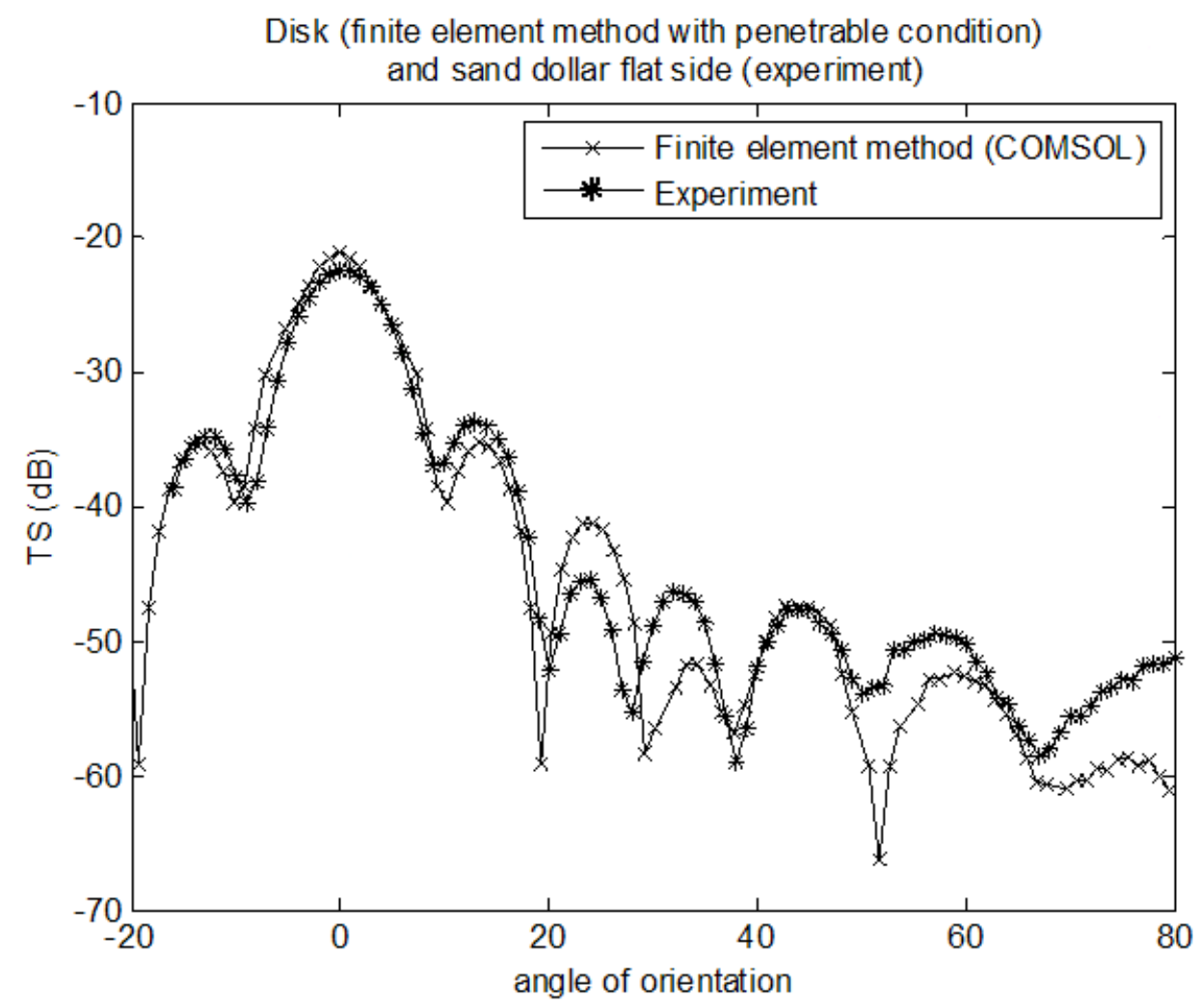

Figure 57 - Comparison of measured target strength at $70 \mathrm{kHz}$ as a function of angle of orientation for the flat side of a sand dollar (solid starred line), to predicted target strength of a penetrable disk $\left(\left|R_{L}\right|\right.$ $=0.4$ ) of radius $3.625 \mathrm{~cm}$ and thickness $5.5 \mathrm{~mm}$ using the finite element method in COMSOL (thin solid line with $x^{\prime}$ s). EPW $=6$ and SMD $=20$.

Both model predictions are greatly improved with the heuristic correction that accounts, in part, for the rigid/fixed boundary conditions. The Kirchhoff prediction is very good near broadside now with the choice of reflection coefficient. The finite element method manages not only to capture the main lobe but also the first pair of side lobes. The finite element method is higher than the corrected experimental curve because of the reflection coefficient chosen. The slight difference in width of the main lobes in figure 57 is likely a result of the choice of radius 
for the disk in the finite element method solution. Both predictions, especially the finite element solution, agree with the data at higher angles of orientation also.

\subsubsection{Sand Dollar Round Side}

For the round side of the sand dollar, the value of the reflection coefficient $\left|R_{L}\right|=0.4$ was chosen in order to match that of the flat side. Figure 58 shows the numerical solution of the Kirchhoff integral for the sand dollar round side with the penetrable condition and the experimental scattering results from the sand dollar round side.

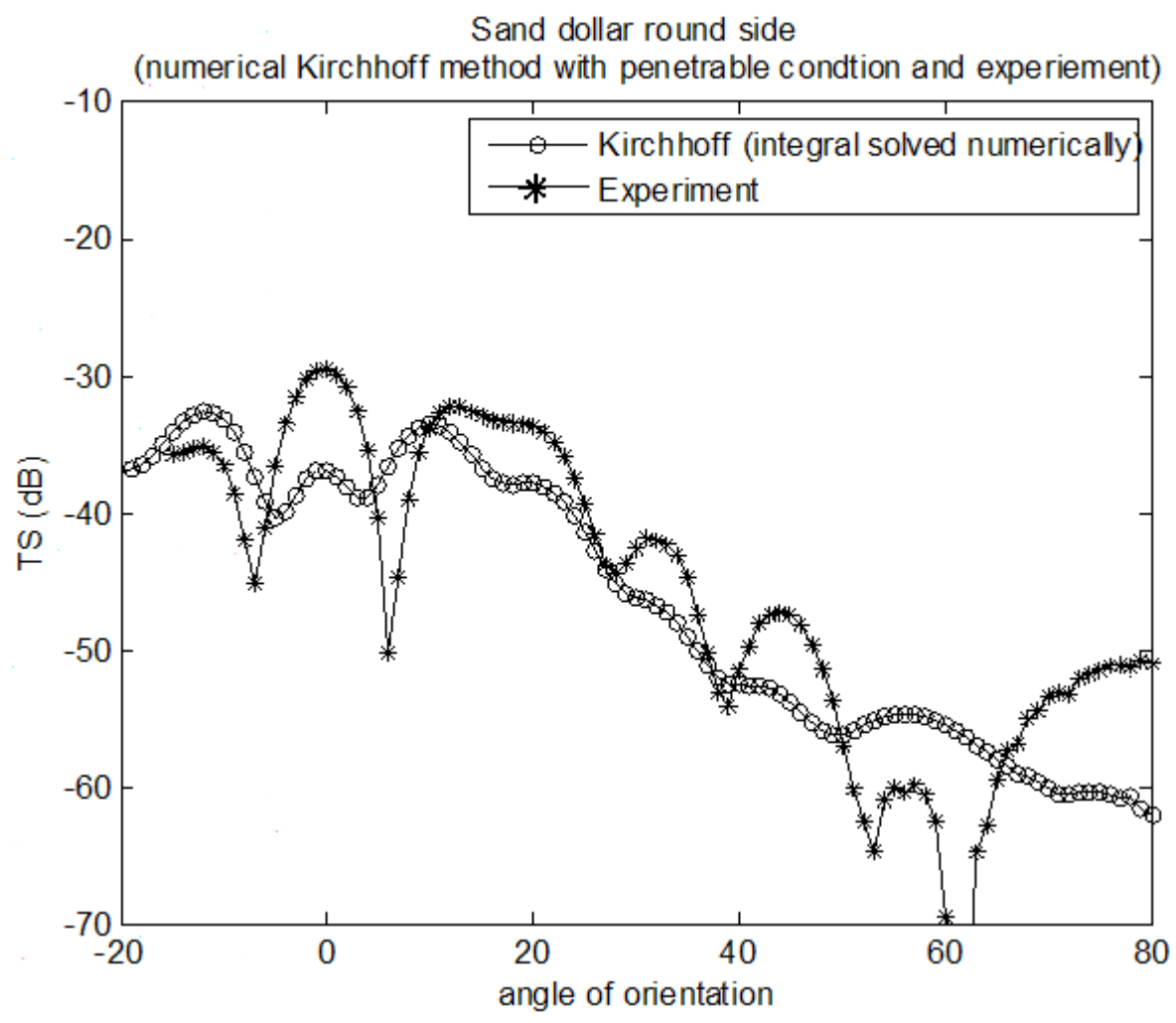

Figure 58 - Comparison of measured target strength at $70 \mathrm{kHz}$ as a function of angle of orientation for the round side of a sand dollar (solid starred line), to predicted target strength of the round side of a penetrable sand dollar $\left(\left|R_{L}\right|=0.4\right)$ found by solving the Kirchhoff integral numerically (solid line with circles). 
There is obvious structural agreement between the predicted results and the experimental data. The very slight traces of the nulls in the Kirchhoff prediction line up with those in the experimental data. There is still much less structure in the model prediction.

\subsection{Summary of Results}

The scattering models developed in this thesis had varying amounts of success in predicting the experimental data for scattering from aluminum disks and sand dollars. The heuristic correction, which accounted for transmission of the incident wave, was developed and was found to significantly improve these predictions. In the case of the aluminum disk, both the Kirchhoff and finite element methods succeeded in accurately recreating the scattering curves near broadside. The finite element solution was better away from broadside, suggesting that there was strong diffraction around the disk.

In the case of the flat side of the sand dollar, the numerical solution of the Kirchhoff integral proved to be very similar to the analytic solution for a disk. Both the Kirchhoff and finite element methods did a reasonably good job in predicting the peak-null structure of the experimental data over the entire range of angles. The success of the heuristic correction over the entire range of orientations suggests that the sand dollar may potentially be modeled as a fluid scatterer in the future, without the need for elastic considerations. Testing this is an important next step toward understanding the sand dollar's scattering mechanics. The fact that the finite element method did not drastically outperform the Kirchhoff method away from broadside, as it did for the aluminum disk, suggests that the effects of diffraction were less for the sand dollar; however, this is difficult to determine. If it is true that diffraction is not as strong for the sand dollar as the aluminum disks, it would be a result of the geometries and/or 
the material properties of the two objects. Understanding this has implications for the application of using diffraction of benthic shells to detect buried objects at subcritical angles.

The round side of the sand dollar was not modeled as well as the flat side with the Kirchhoff method. Because the finite element method was not tested for this shape, it is difficult to conclusively determine the reasons for this. However, it was seen that the numerical solution to the Kirchhoff integral is a better model than the analytic solution for a spherical cap. This proves the utility of the solving the Kirchhoff integral numerically. Although the results were not as good as for the flat side, the general structures of the prediction and the experimental data are similar. 


\section{Chapter 5 Summary and Conclusion}

This focus of this thesis is to understand the acoustic backscattering from individual sand dollars (Dendraster excentricus) in the free field. The following paragraphs summarize the motivations for this work, the research performed, recommendations for future work, and the contributions of this thesis.

\subsection{Motivation}

Knowledge of seafloor scattering has many important applications. Benthic shelled organisms have been shown to be important contributors to scattering from the seafloor in certain cases, depending on the acoustic frequency, angle of incidence, and density of organisms. Understanding the scattering physics of individual benthic shells is an important step toward understanding the effects of collections in the ocean. Sand dollars were chosen for this thesis because of existing laboratory data to which the models could be compared.

\subsection{Modeling}

Acoustic scattering models can provide insight to the scattering mechanisms from complex objects, such as sand dollars. The Kirchhoff method is a very simple model that proves to be very useful at high frequencies (short wavelengths relative to the object size). The numerical method of solving the Kirchhoff integral developed here greatly broadens this approach by allowing the scattering from irregularly shaped objects to be predicted. It is 
believed that the accuracy of predictions for an object's scattering can be greatly increased by solving the integral numerically, as opposed to solving it analytically for a simplified object, as was the sand dollar's round side. Applied to acoustic scattering problems, the finite element method is a technique far more complicated than the Kirchhoff method. It requires powerful computer resources for any meaningful three dimensional calculations. Its advantage is that it does not make any of the approximations implicit in the Kirchhoff method, such as neglecting the effects of diffraction. However, it has been seen that at high enough frequencies near broadside, the finite element method's predictions are of about the same accuracy as those from the Kirchhoff method. It is believed that the finite element method's value in acoustic scattering problems lies at lower frequencies. The heuristic correction for the rigid/fixed boundary condition that was developed here was shown to improve the ability of the model to predict the experimental scattering data.

\subsection{Recommendations for Future Work}

One main limitation of this work was the use of a rigid/fixed boundary condition in the scattering models. This allowed only "first order" approximations to the scattering from the elastic objects to be studied. Without incorporating the elasticity of a target, it is difficult to distinguish the importance of certain scattering phenomena such as diffraction, surface elastic waves, and shear wave effects. Although the Kirchhoff method is unable to incorporate the effects of elasticity, the same is not true for the finite element method. Currently, a finite element code is being developed at the Woods Hole Oceanographic Institution which would allow for the modeling of elastic domains (Gonzalo Feijoo, personal communication). This code will also incorporate a perfectly matched layer, a far better radiation condition than the 
simplified Sommerfeld condition used in the COMSOL simulations (Berenger 1994, 1996; Harari et al., 2000). It would be valuable to test scattering predictions for sand dollars or other benthic shells with the ability to model their elasticity. In addition, this study concerned sand dollars in the free field. Scattering involving the geometry where sand dollars are on or near a boundary should be examined. As sand dollars will generally be encountered in the ocean lying on the seafloor, modeling their scattering while near a boundary could help gain additional insight. This could be performed in the finite element method by dividing the surrounding medium domain into two fluid regions, one representing the seawater and one representing the seafloor.

The comparisons between model predictions and experimental data in this thesis focused on a single frequency over a range of angles. The insight that these comparisons offer could be bolstered by further comparisons with more experimental data. Experimental data for additional sand dollars would reduce the effects of experimental error as well as provide insight to their scattering physics by examining differences between samples. Comparisons to model predictions at frequencies other than $70 \mathrm{kHz}$ would aid this understanding as well. As a final experimental recommendation, the scattering from a live sand dollar should be studied. It is unknown how the scattering physics of such an organism, surrounded by a thin layer of tissue, would be different from only the test.

Sand dollars are often found in dense aggregates in situ, creating a very complicated scattering problem. The hope is that the study of scattering from individual sand dollars in the free field leads to a greater understanding of how these aggregates of organisms scatter sound in the ocean. 


\subsection{Contributions of Thesis}

- Development of analytic Kirchhoff method scattering models for a sand dollar, namely a disk and spherical cap.

- Development of a numerical technique for solving the Kirchhoff integral for complex three dimensional objects.

- Comparison of the analytic and numerical solutions of the Kirchhoff integral with experimental scattering data from a sand dollar and aluminum disk.

- Application of the finite element method in the program COMSOL for predicting the scattering from a rigid/fixed disk. Comparison of finite element method model predictions with experimental scattering data from a sand dollar and aluminum disk.

- Development of a heuristic approach for accounting for a penetrable boundary condition for high aspect ratio objects at broadside. Application of this approach for the Kirchhoff and finite element method predictions for scattering from a sand dollar and aluminum disk. 


\section{Appendix A: MATLAB Code}

One way to solve the Kirchhoff integral numerically is by generating a mesh in

COMSOL Multiphysics. The object must be oriented so that the incident plane wave is traveling

in the negative $\mathrm{z}$ direction. Once the geometry and the mesh have been created, they can be exported to COMSOL script as a fem structure. The function kirchhoff_mesh.m was written for

COMSOL Multiphysics Script (ver. 1.0a) to extract the surface mesh information and save to file its connectivity and coordinate information.

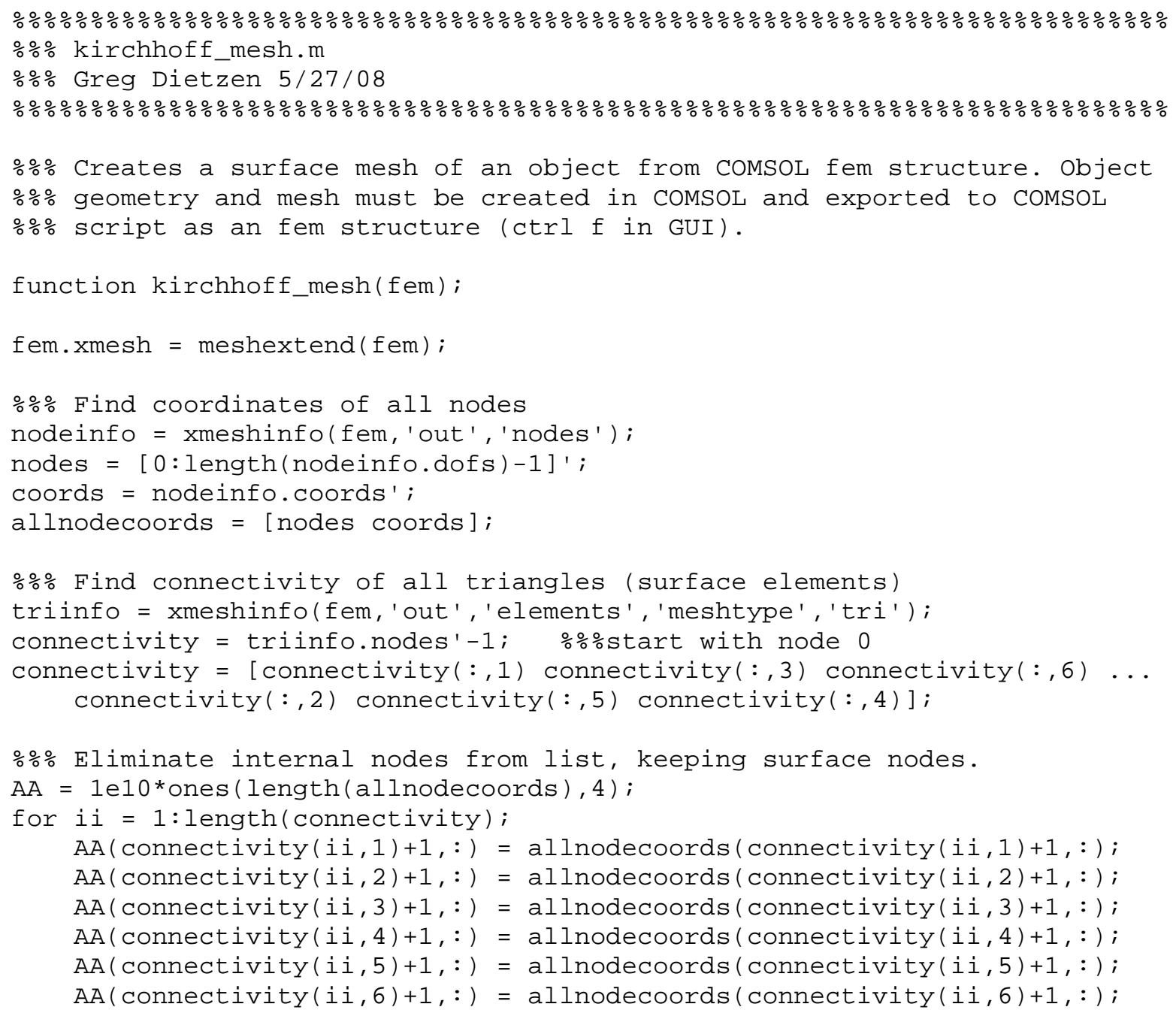




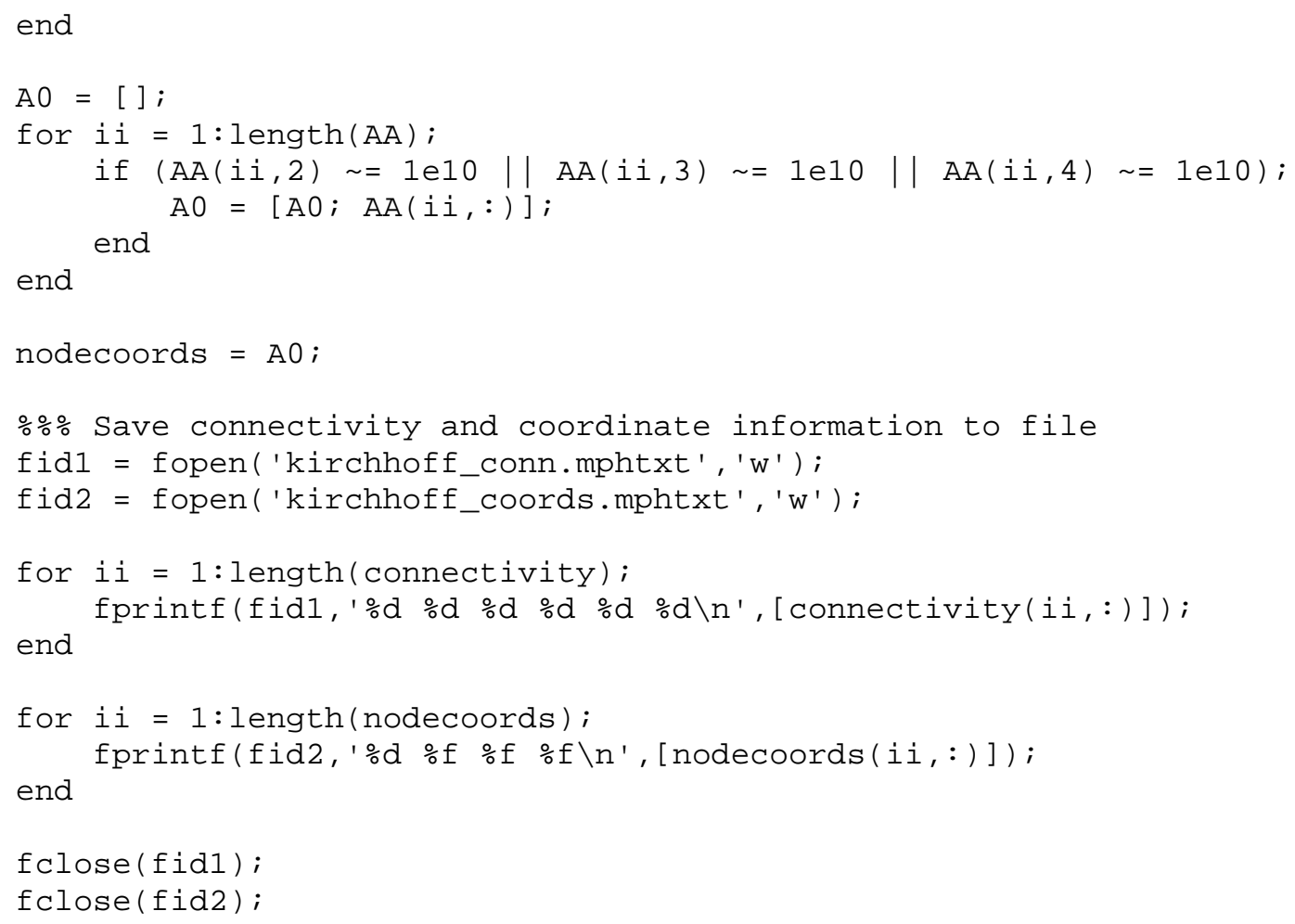

After the surface mesh files have been generated, they can be read to evaluate the Kirchhoff integral using the functions kirchhoff_integral.m, newell.m, and trianglepoint.m, written for use in MATLAB (ver. 7.2.0.232). The inputs are the surface mesh file names and the wavenumber of the incident plane wave.

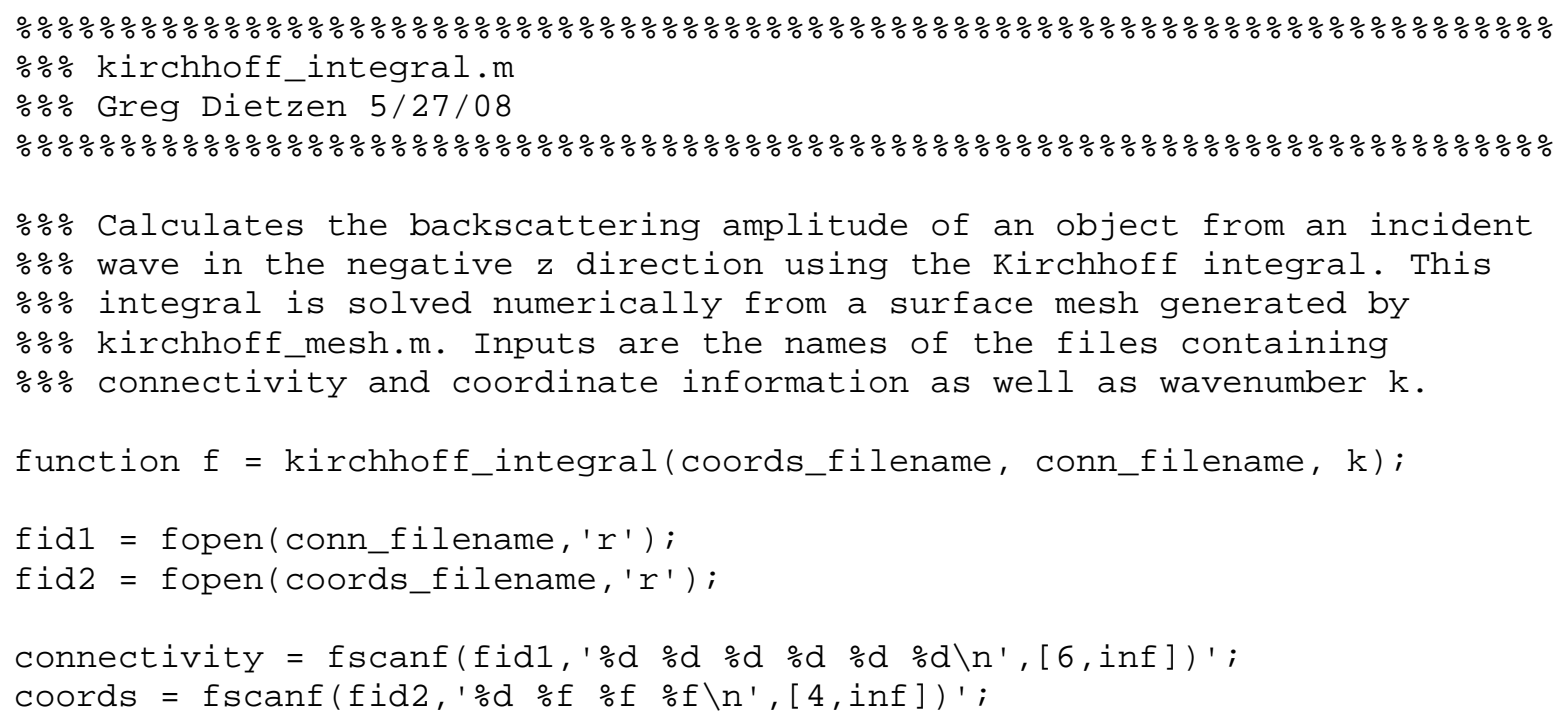




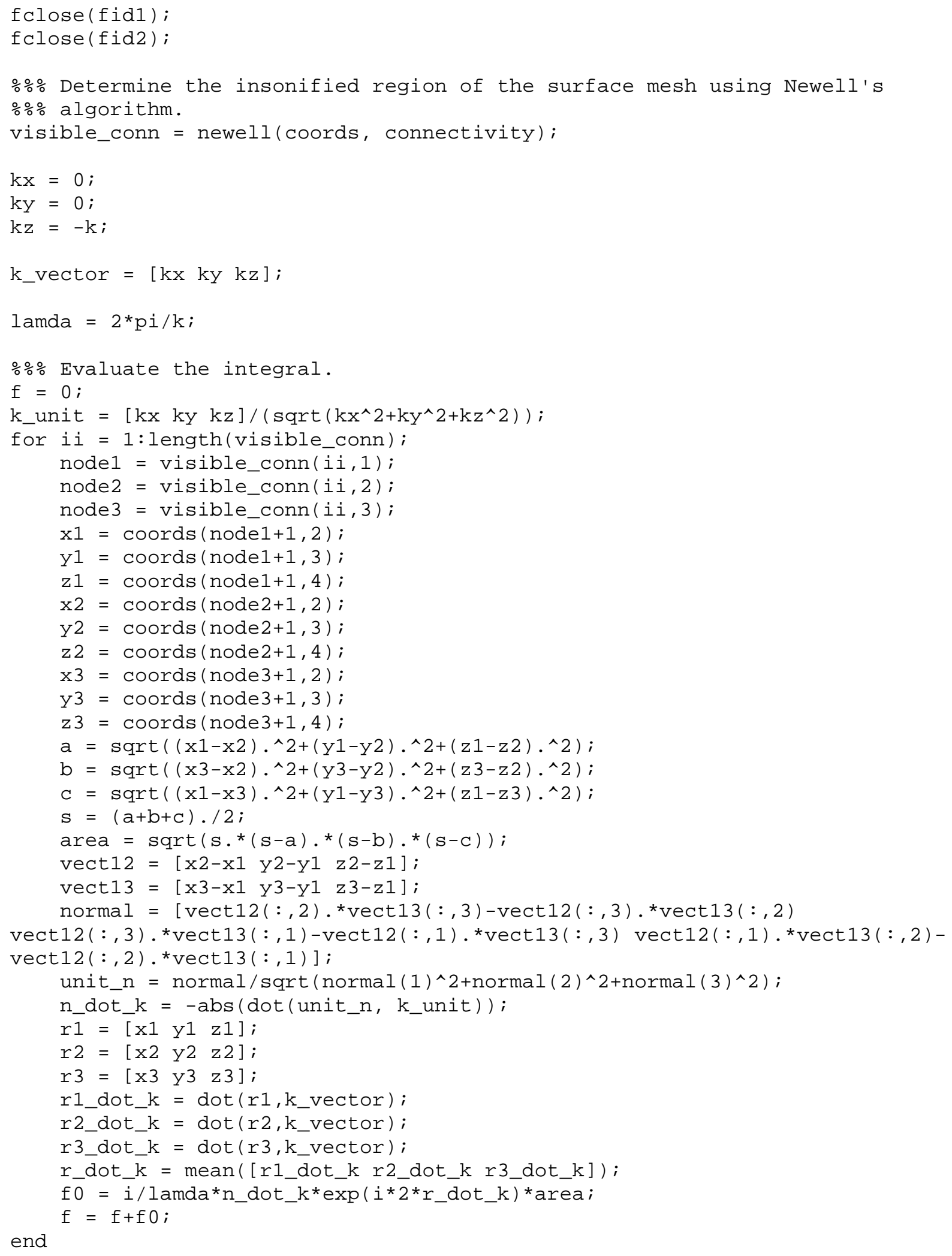




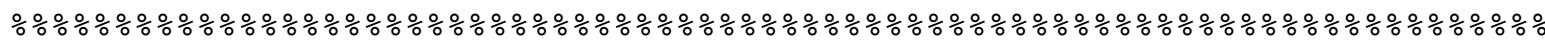

응응 newell.m

응응 Greg Dietzen 5/27/08

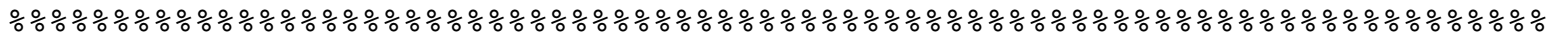

\%응 Determines the insonified region of a surface mesh using a modified

응 Newell's algorithm. Incoming wave is in the negative z direction.

function visible_conn = newell (coordinates, connectivity);

warning off MATLAB:divideByzero

응 Number triangular elements for labeling purposes.

connectivity $=[[0:$ length (connectivity) -1$]$ ' connectivity $]$;

응 Sample size for rasterization test.

samplesize $=20$;

응응 Determine min and $\max x, y$, and $z$ values for each element and sort by $\circ \% \max \mathrm{z}$.

maxmin $=$ zeros (length (connectivity), 7);

for $i i=1:$ length (connectivity);

node1 = connectivity $(i i, 2)$;

node2 = connectivity $(i i, 3)$;

node $3=$ connectivity $(i i, 4)$;

$\operatorname{maxx}=\max ([\operatorname{coordinates}($ node $1+1,2)$ coordinates (node $2+1,2) \ldots$ coordinates (node $3+1,2)]$ );

$\operatorname{maxy}=\max ([\operatorname{coordinates}($ node $1+1,3)$ coordinates $($ node $2+1,3) \ldots$ coordinates (node $3+1,3)])$;

$\max z=\max ([\operatorname{coordinates}($ node $1+1,4)$ coordinates $($ node $2+1,4) \ldots$ coordinates (node $3+1,4)])$;

$\operatorname{minx}=\min ([\operatorname{coordinates}($ node $1+1,2)$ coordinates $($ node $2+1,2) \ldots$ coordinates (node $3+1,2)])$;

miny $=\min ([\operatorname{coordinates}($ node $1+1,3)$ coordinates $($ node $2+1,3) \ldots$ coordinates (node $3+1,3)])$;

$\operatorname{minz}=\min ([\operatorname{coordinates}($ node $1+1,4)$ coordinates $($ node $2+1,4) \ldots$ coordinates (node3+1,4)]);

end

maxmin(ii,:) $=[i i-1$ minx maxx miny maxy minz maxz];

sortmaxmin $=$ sortrows (maxmin, 6);

sortmaxmin $=$ flipud (sortrows $(\operatorname{sortmaxmin}, 7))$;

응 Minmax tests in $\mathrm{x}$ and $\mathrm{y}$ and rasterization test.

i $=1$;

while ii <= length (sortmaxmin);

$\operatorname{maxx}=$ sortmaxmin $(i i, 3)$;

$\operatorname{minx}=$ sortmaxmin $(i i, 2) ;$

maxy $=$ sortmaxmin $(i i, 5)$;

miny $=$ sortmaxmin $(i i, 4)$;

$j j=i i+1$;

while $j j<=$ length (sortmaxmin);

$\operatorname{maxx} 2=\operatorname{sortmaxmin}(j j, 3)$;

$\operatorname{minx} 2=\operatorname{sortmaxmin}(j j, 2)$;

if $(\min x>=\operatorname{maxx} 2|| \max x<=\operatorname{minx} 2)$;

else

$j j=j j+1$;

maxy2 $=$ sortmaxmin $(j j, 5) ;$ 


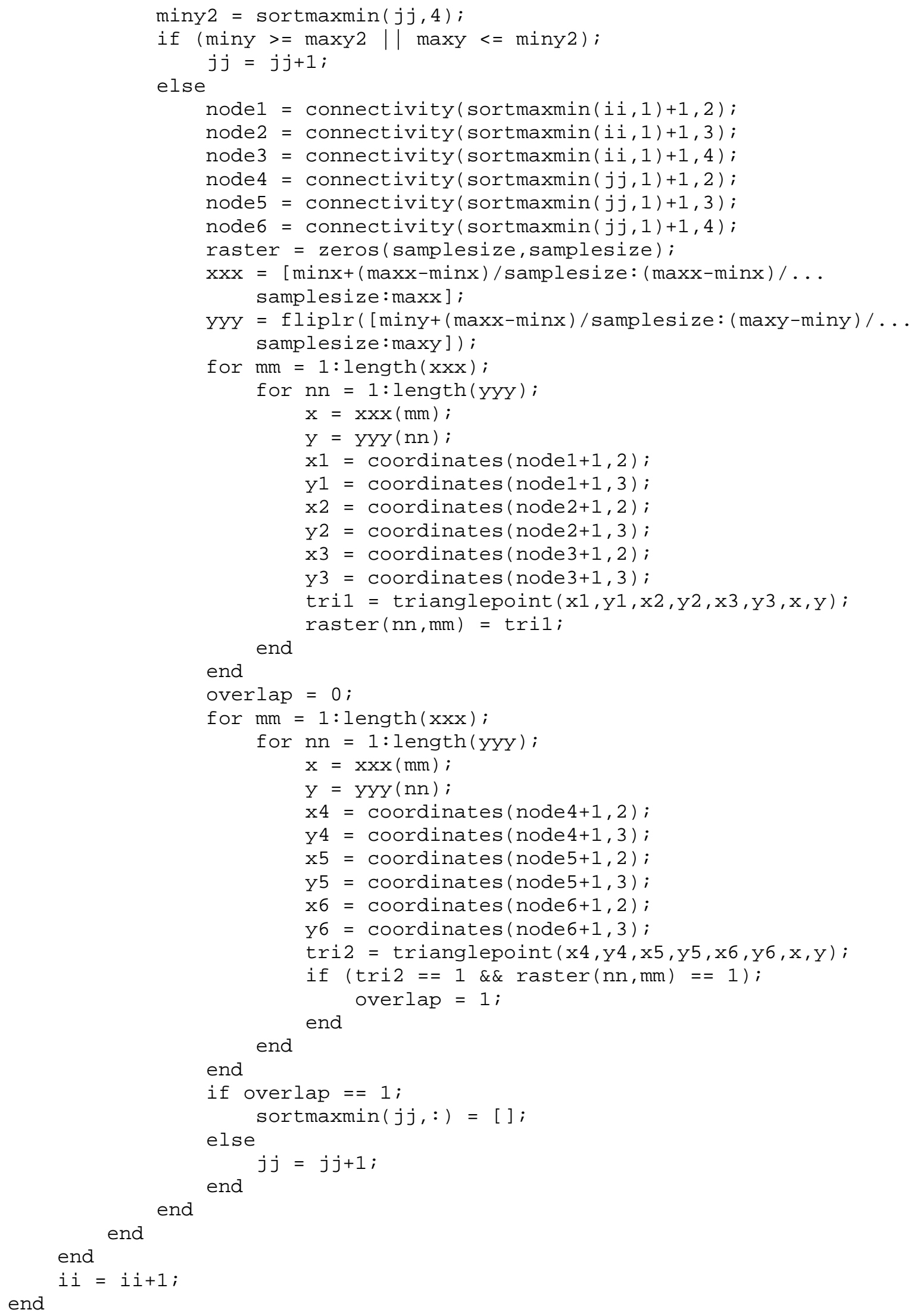




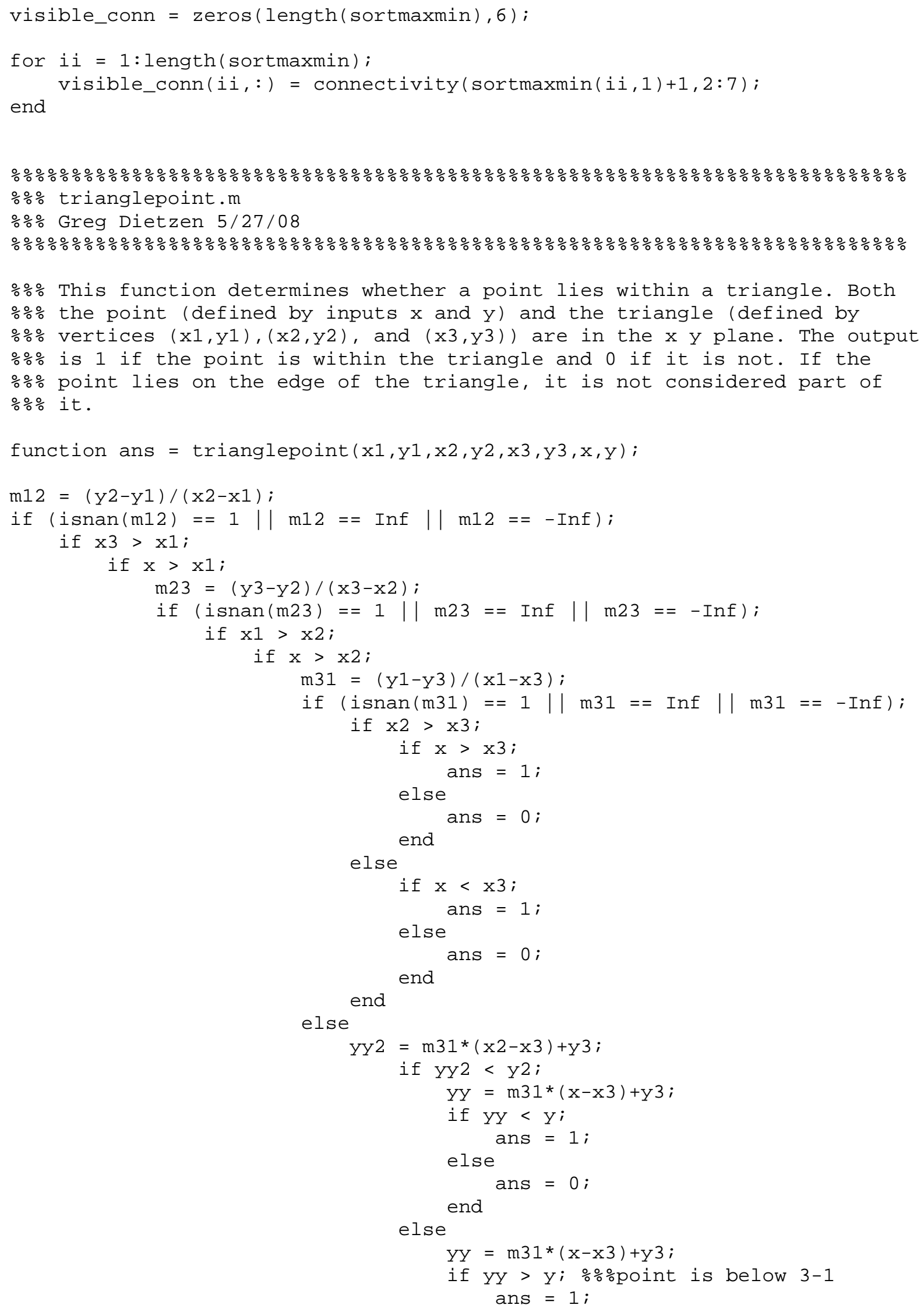




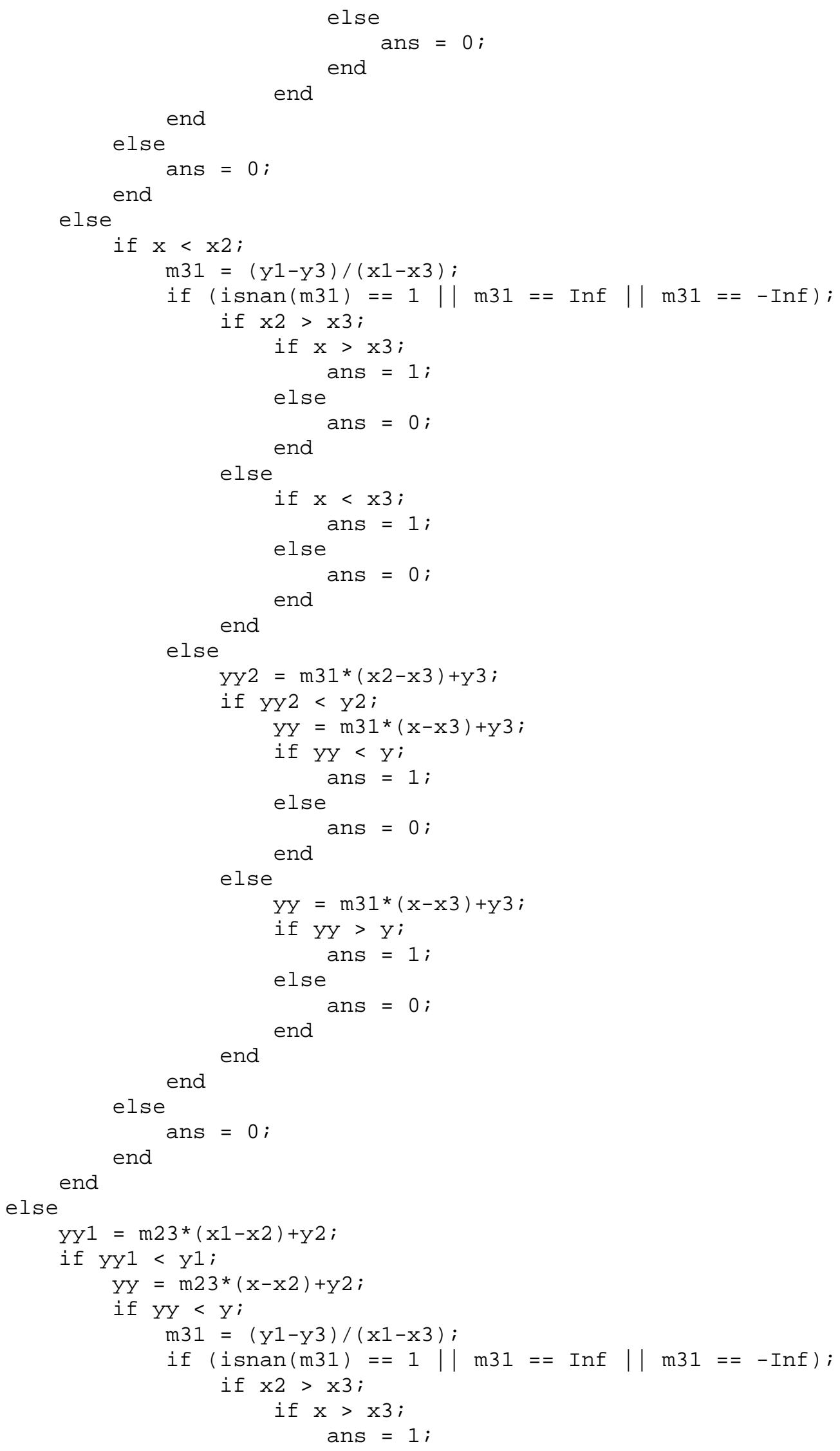




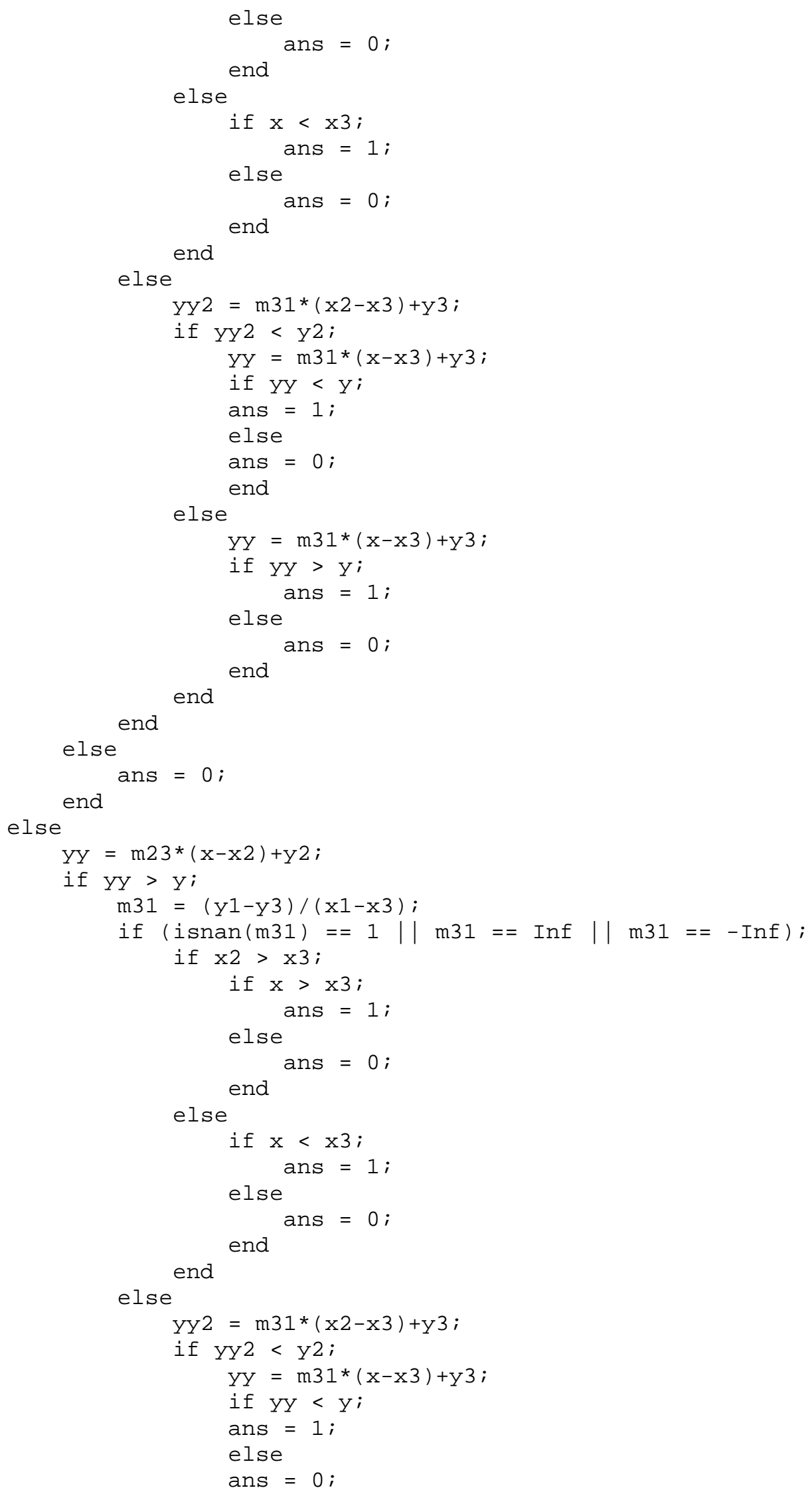




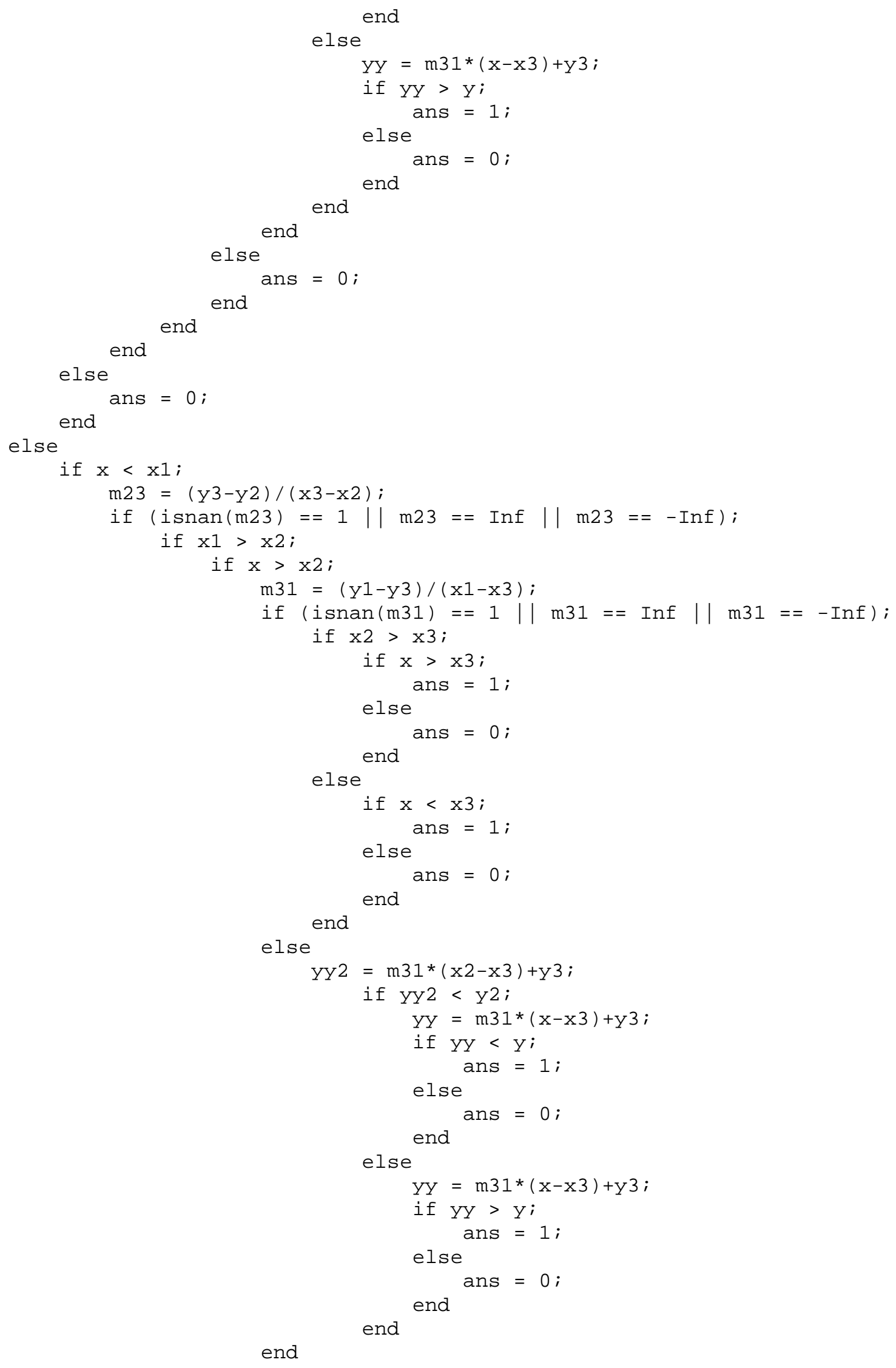




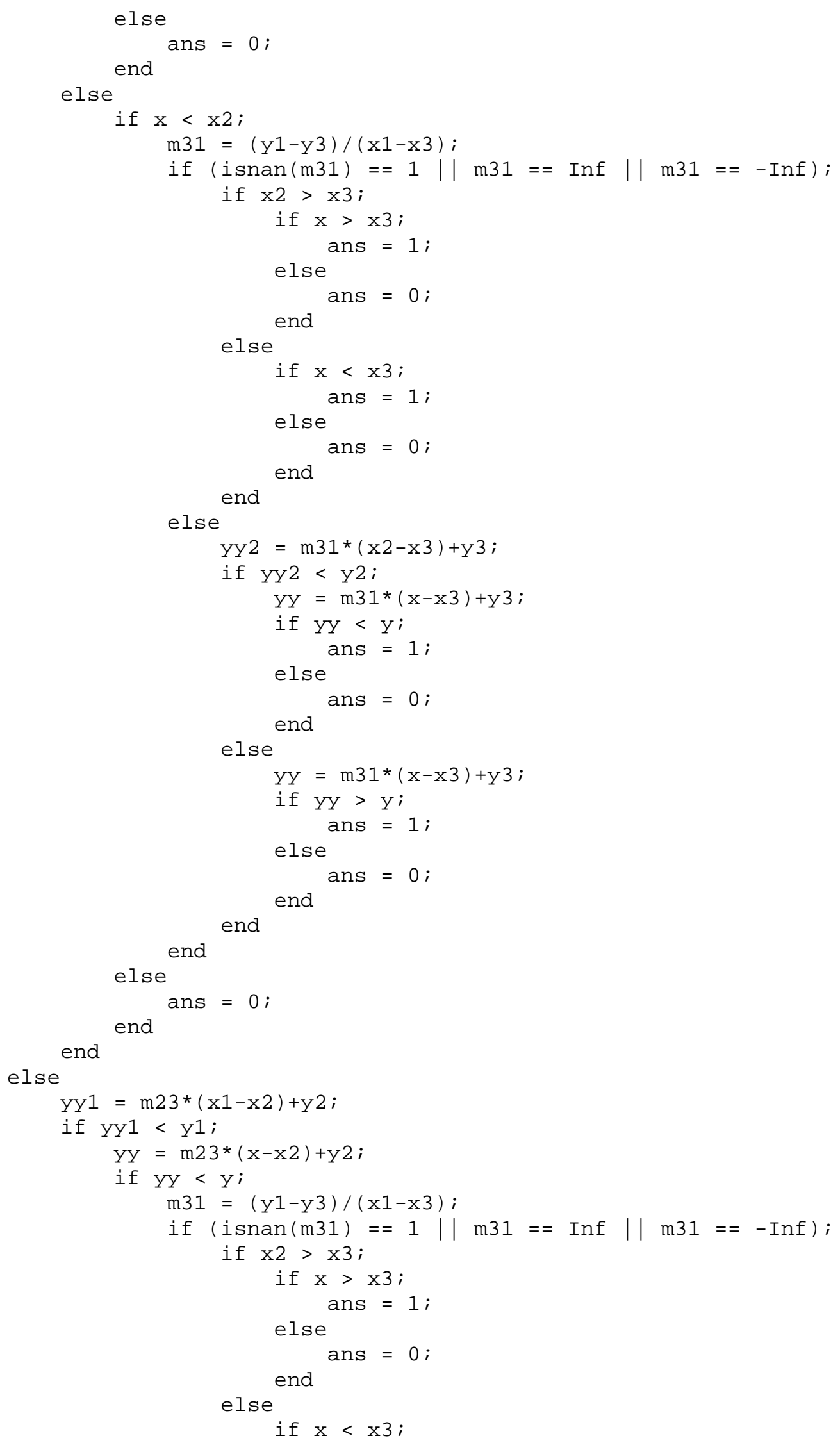




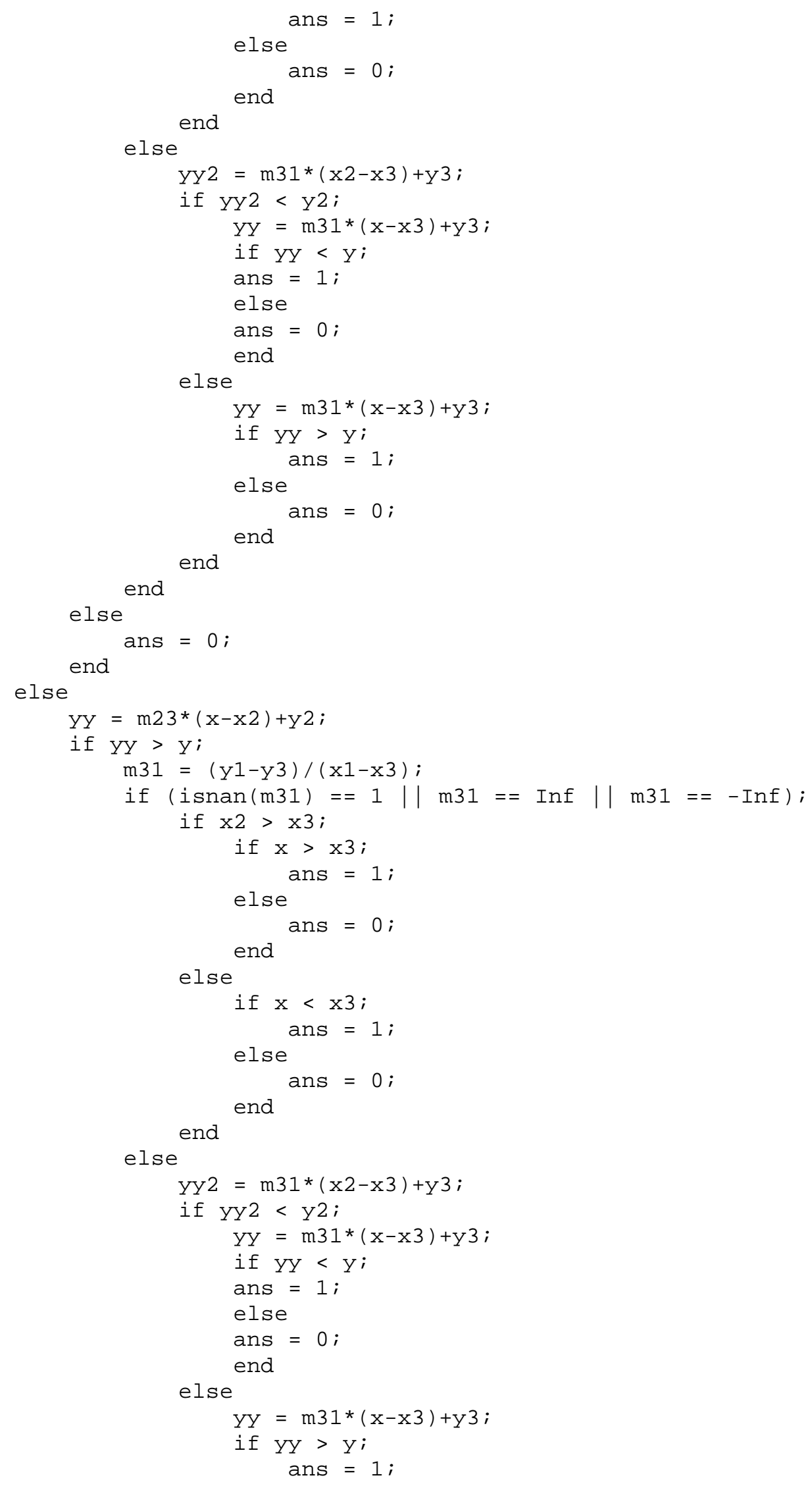




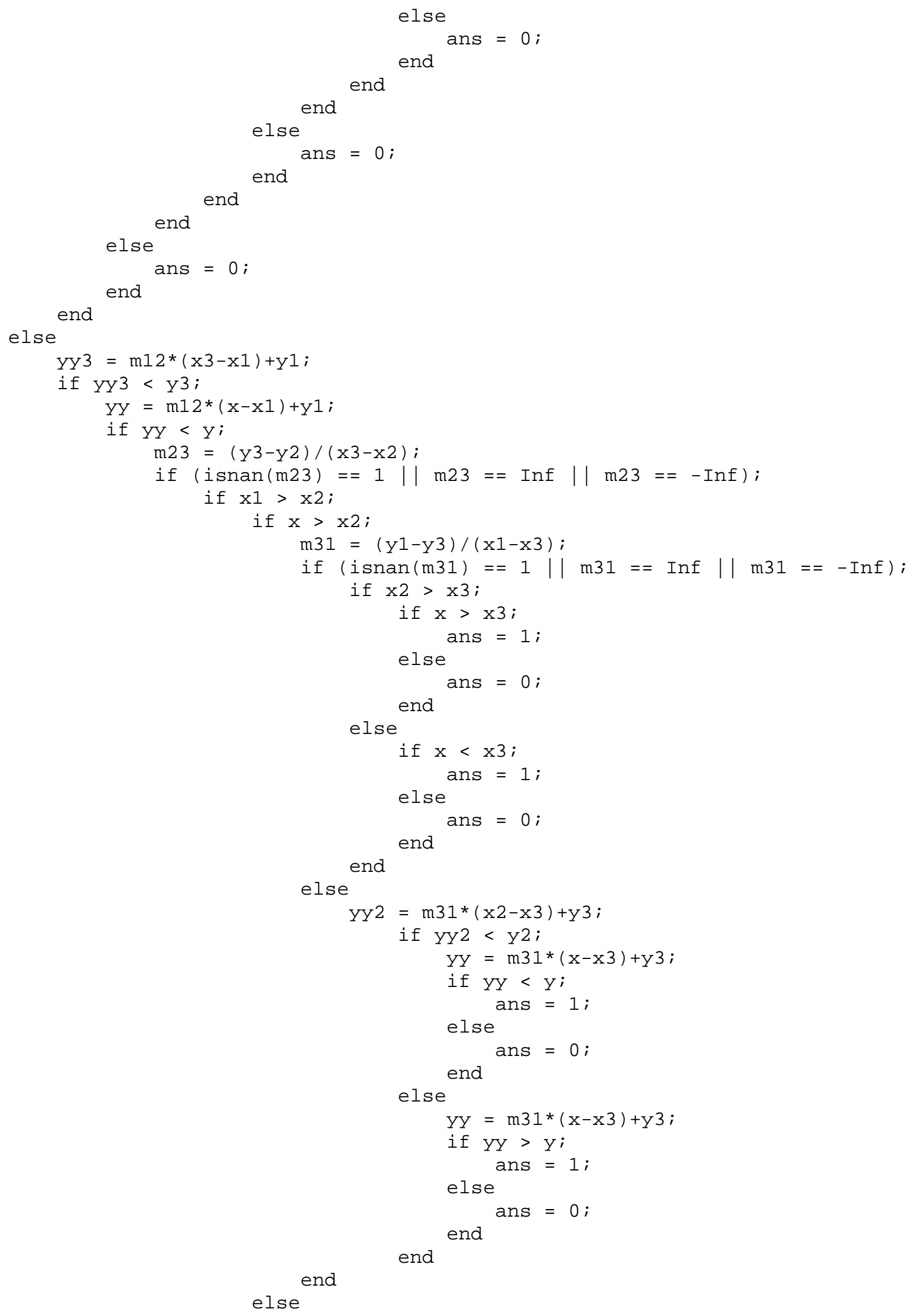




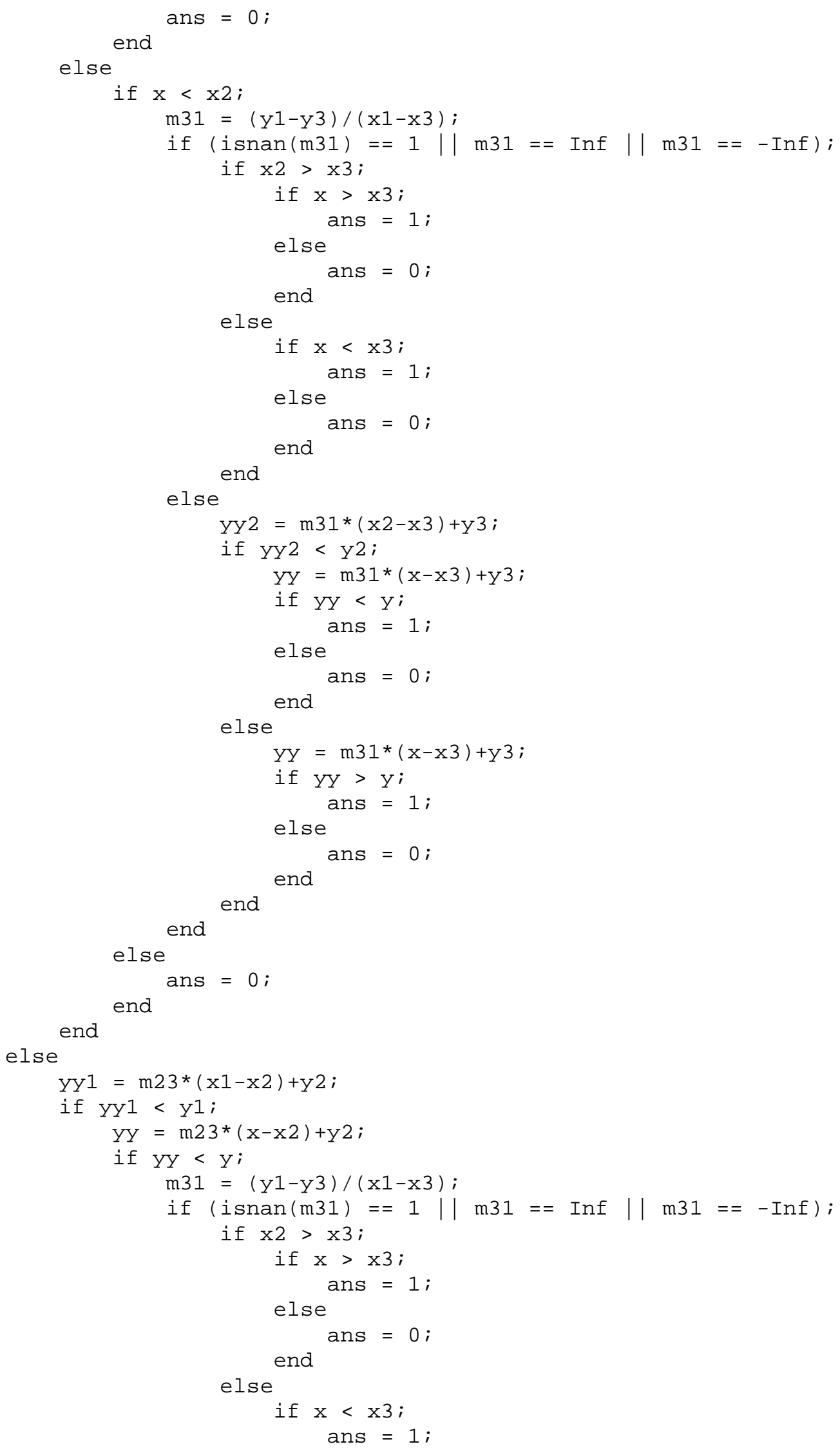




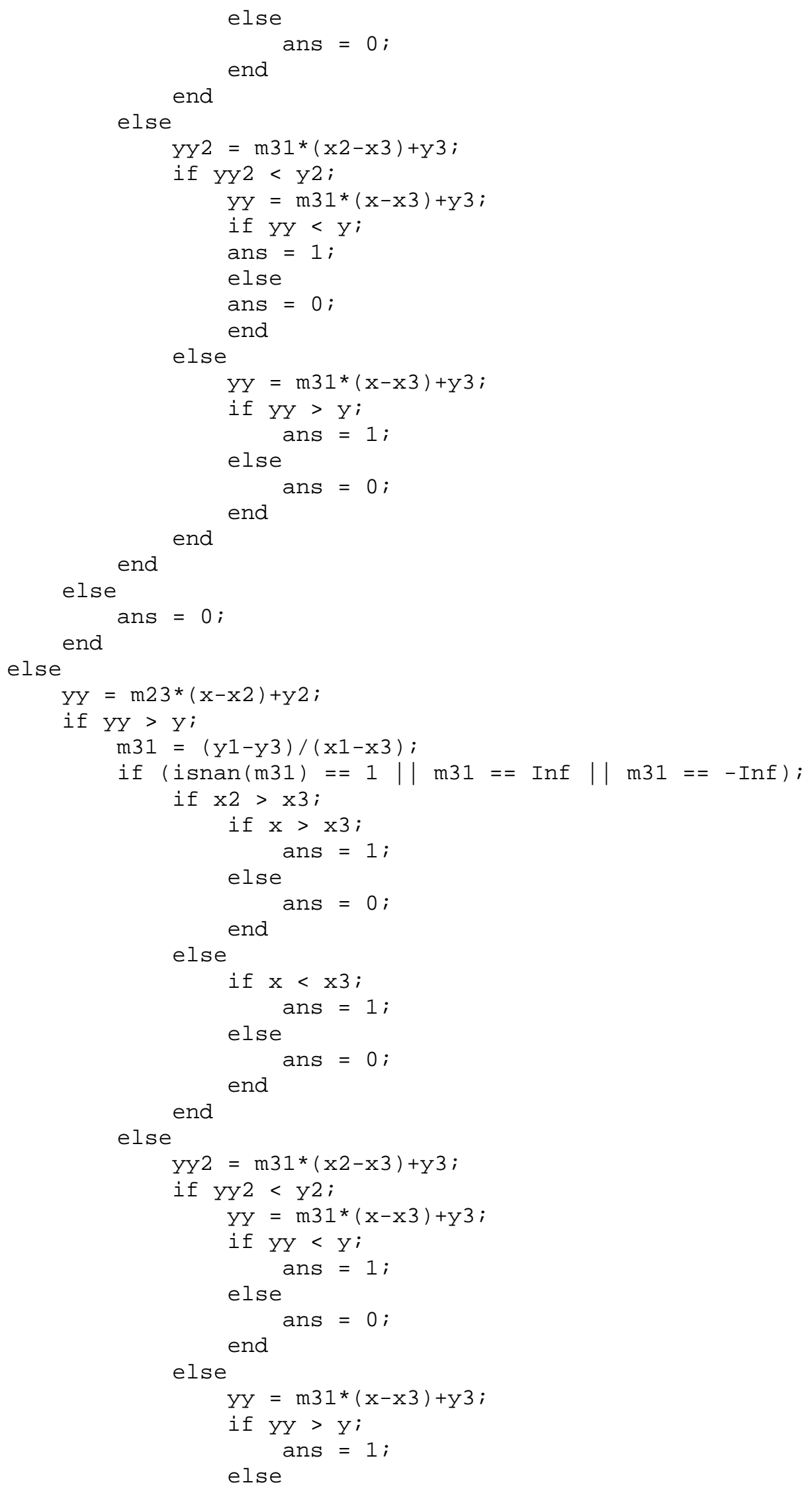




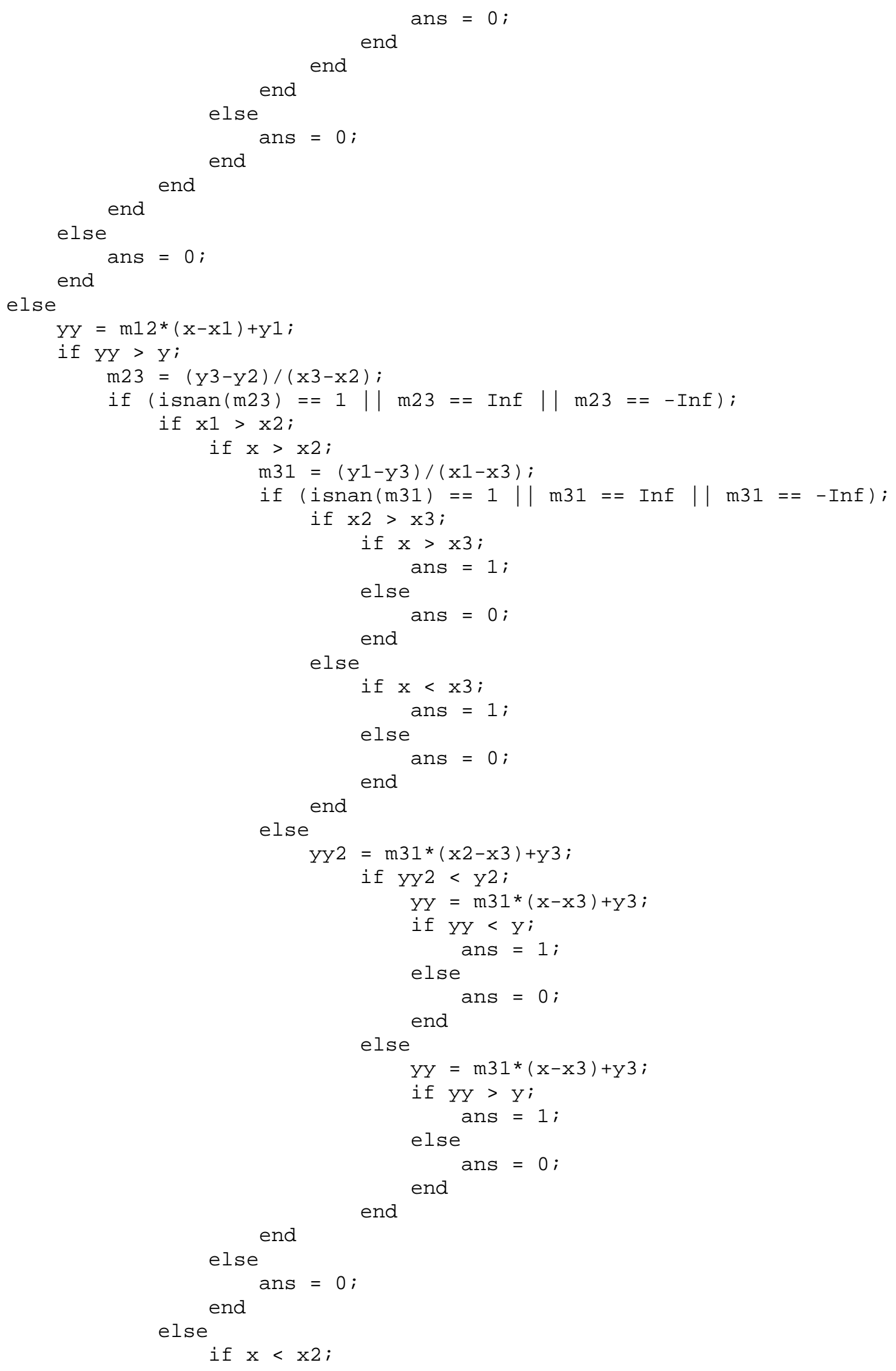




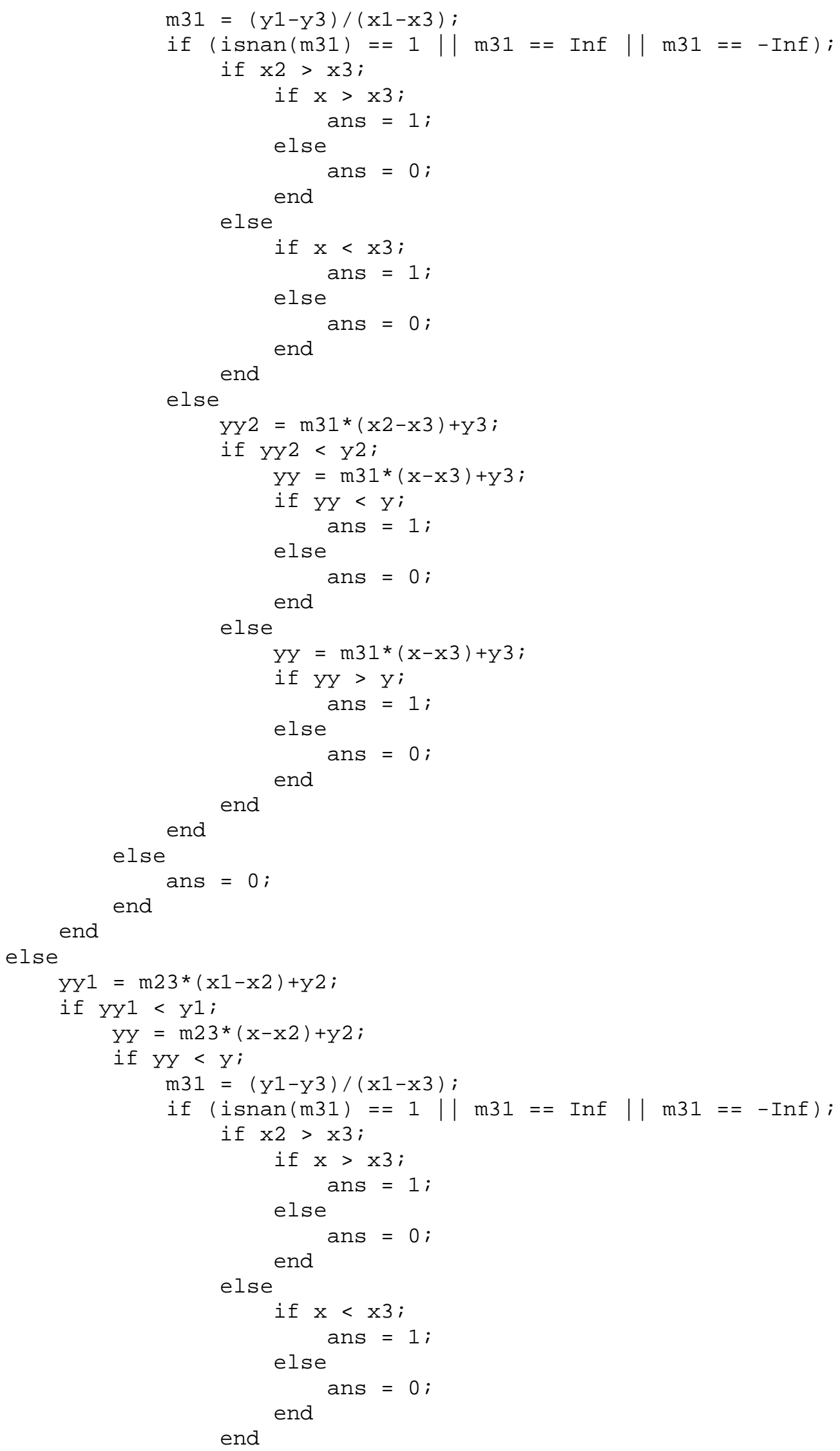




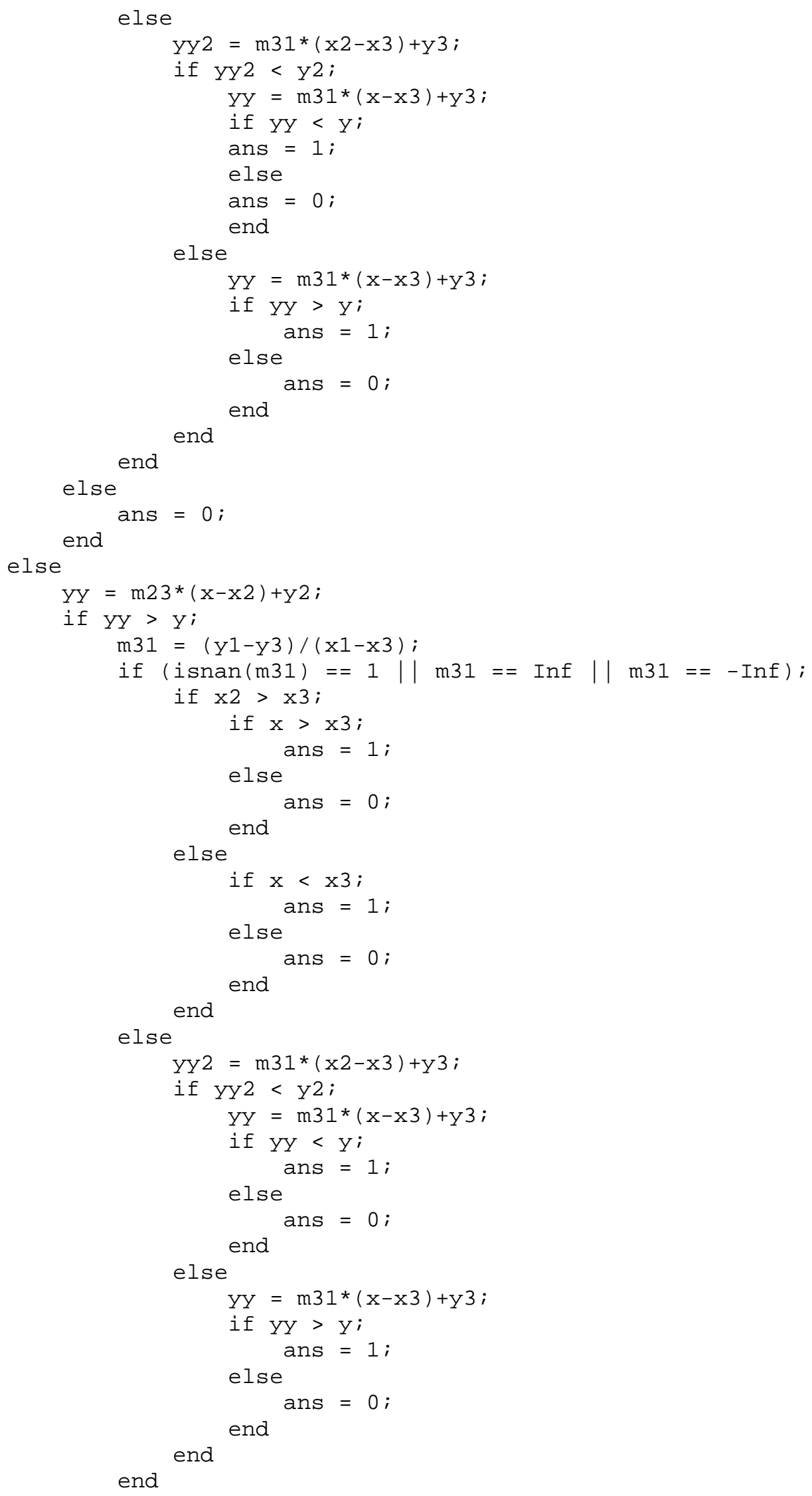




$$
\begin{aligned}
& \text { else ans = o; } \\
& \text { end end end } \\
& \text { else ans }=0 ; \\
& \text { end end }
\end{aligned}
$$




\section{Bibliography}

Abramowitz, M. and Stegun, I. A. (1965). Handbook of Mathematical Functions (Dover Publications, New York).

Anderson, V. C. (1950). "Sound scattering from a fluid sphere", J Acoust Soc Amer 22, 426-431.

Atkinson, F. V. (1949). “On Sommerfeld's radiation condition”, Philos Mag 40, 645-651.

Babuska, I. and Aziz, A. K. (eds). (1973). The Mathematical Foundations of the Finite Element Method - with Applications to Partial Differential Equations, (Academic Press, New York).

Becker, E., Carey, G., and Oden, J. (1981). Finite Elements. An Introduction. Vol. 1 (Prentice-Hall, Englewood Cliffs, NJ).

Berenger, J. P. (1994). “A perfectly matched layer for the absorption of electromagnetic waves", J Comput Phys 114, 185-200.

Berenger, J. P. (1996). “Three-dimensional perfectly matched layer for the absorption of electromagnetic waves", J Comput Phys 127, 363-379.

Bettess, P. (1977). “Infinite elements”, Int J Numer Methods Eng 11, 53-64.

Born, M. and Wolf, E. (1970). Principles of Optics (Academic, New York).

Bowman, J. J., Senior T. B. A., and Uslenghi, P. L. E. (1969). Electromagnetic and Acoustic Scattering Simple Shapes (North-Holland, Amsterdam).

Brekhovskikh, L. M. and Lysanov, Y. P. (2003). Fundamentals of Acoustics, 3rd edition (Springer-Verlag, New York).

Carmichael, R. S. (1982). CRC Handbook of Physical Properties of Rocks, Volume II (CRC, Boca Raton, FL).

Chia, F. S. (1969). "Some observations on the locomotion and feeding of the sand dollar, Dendraster excentricus (Eschscholtz)", Journal of Exper Marine Bio and Eco 3, 162-170.

Chu, D., Foote, K G., and Stanton, T. K. (1993). “Further analysis of target strength measurements of Antarctic krill at 38 and $120 \mathrm{kHz}$ : comparison with deformed cylinder model and inference of orientation distribution", J Acoust Soc Amer 93, 2985-2988. 
Clough, R. W. (1960). “The finite element method in plane stress analysis”, Proceedings of 2nd ASCE Conference on Electronic Computation, Pittsburgh, PA, Sep 8 and 9.

Courant, R. (1943). "Variational methods for the solutions of problems of equilibrium and vibrations", Bull Am Math Soc 49, 1-23.

Craggs, A. (1972). "The use of simple three-dimensional acoustic finite elements for determining the natural modes and frequencies of complex shaped enclosures", J Sound Vib 23, 331-339.

De Berg, M., Van Krevald, M., Overmars, M., and Schwarzkopf, O. (1997). Computational Geometry (Springer, New York).

Faran, J. J. (1951). "Sound scattering by solid cylinders and spheres", J Acoust Soc Amer 23, 405-418.

Fenstermacher, L. E., Crawford, G. B., Borgeld, J. C., Britt, T., George, D.A., Klein, M. A., Driscoll, N. W., and Mayer, L. A. (2001). "Enhanced acoustic reflectivity due to high abundance of sand dollars, Dendraster excentricus", Marine Georesources Geotechnol 19, 135-145.

Franz, W. (1957). Theorie der beugung elektromagnetischer Wellen (Springer-Verlag, Berlin, 1957).

Frisk, G. V. (1994). Ocean and Seabed Acoustics (Prentice-Hall, Englewood Cliffs, NJ).

Gan, H., Levin, P. L., and Ludwig, R. (1993). "Finite element formulation of acoustic scattering phenomena with absorbing boundary condition in the frequency domain", J Acoust Soc Amer 94, 1651-1662.

Gaunaurd, G. C. (1985). “Sonar cross sections of bodies partially insonified by finite sound beams", IEEE J Ocean Eng 10, 213-230.

Gladwell, G. M. L. (1966). “A variational formulation of damped acoustostructural vibration problems", J Sound Vib 4, 172-186.

Golub, G. H. and Van Loan, C. F. (1996). Matrix Computations (JHU Press, Baltimore, $\mathrm{MD})$.

Greenlaw, C. F. (1977). "Backscattering spectra of preserved zooplankton”, J Acoust Soc Amer 62, 44-52. 
Harari, I., Slavutin, M, and Turkel, E. (2000). "Analytical and numerical studies of a finite element PML for the Helmholtz equation", J Comp Acoust 8, 121-137.

Hefner, B. T. and Marston, P. L. (2001). "Backscattering enhancements associated with the excitation of symmetric Lamb waves on a circular plate: direct and holographic observations", Acou Res Let Online 2, 55-60.

Highsmith, R. C. (1982). "Induced settlement and metamorphosis of sand dollar (Dendraster excentricus) larvae in predator-free sites: adult sand dollar beds", Ecology 63, 329-337.

Huebner, K. H. (1975). The Finite Element Method for Engineers (Wiley-Interscience, New York).

Hunt, J. T., Knittel, M. R., and Barach, D. (1974). “Finite-element approach to acoustic radiation from elastic structures", J Acous Soc Am 55, 369-280.

Hunt, J. T., Knittel, M. R., Nichols, C. S., and Barach, D. (1975). “Finite-element approach to acoustic scattering from elastic structures", J Acoust Soc Amer 57, 287-299.

Jackson, D. R., Baird, A. M., Crisp, J. J., Thomson, P. A. G. (1986). “High frequency bottom backscatter measurements in shallow water", J Acoust Soc Amer 80, 1188-1199.

Johnson, R. K. (1977). “Sound scattering from a fluid sphere revisited", J Acoust Soc Amer 62, 375-377; "Erratum: 'Sound scattering from a fluid sphere revisited'”, J Acoust Soc Amer 63, 626 (1978).

Kristensson, G. and Waterman, P. C. (1982). "The T matrix for acoustic and electromagnetic scattering by circular disks", J Acoust Soc Amer 72, 1612-1625.

Lavery, A. C., Stanton, T. K., McGehee, D. E., and Chu, D. (2002). “Three-dimesional modeling of acoustic backscattering from fluid-like zooplankton", J Acoust Soc Amer $111,1197-1210$.

Lavery, A. C., Wiebe, P. H., Stanton, T. K., Lawson, G. L., Benfield, M. C., and Copley, N. (2007). "Determining dominant scatterers of sound in mixed zooplankton populations", J Acoust Soc Amer 122, 3304-3326.

Lawson, G. L., Wiebe, P. H., Ashjian, C. J., Chu, D., and Stanton, T. K. (2006). “Improved parameterization of Antarctic krill target strength models", J Acoust Soc Amer 119, 232242. 
Medwin, H. and Clay, C. S. (1998). Fundamentals of Acoustical Oceanography (Academic Press, New York).

Molz, E. B. and Beamish, J. R. (1995). “Leaky plate modes: radiation into a solid medium", J Acoust Soc Amer 99, 1894-1900.

Nefske, D. J., Wolf, J. A., and Howell, L. J. (1982). “Structural-acoustic finite element analysis of the automobile passenger compartment: A review of current practice", J Sound Vib 80, 247-266.

Newell, M. E., Newell, R. G., and Sancha, T. L. (1972). “A new approach to the shaded picture problem", Proc ACM National Conf.

Nichols, D. (1969). Echinoderms, 4th edition (Hutchinson \& Co, London).

Norton, G. V., Novarini, J. C., and Keiffer, R. S. (1993). "An evaluation of the Kirchhoff approximation in predicting the axial impulse response of hard and soft disks", J Acoust Soc Amer 93, 3049-3056.

Petyt, M., Lea, J., and Koopmann, G. H. (1976). “A finite element method for determining the acoustic modes of irregular shaped cavities", J Sound Vib 45, 495-502.

Rayleigh, Lord. (1945). Theory of Sound (Dover, New York).

Schwinger, J., DeRaad, Jr., L. L., Milton, K. A., and Tsai, W. (1998). Classical Electrodynamics (Perseus Books, Reading, MA).

Simmonds, J. and MacLennan, D. (2005). Fisheries Acoustics, 2nd edition (Blackwell, Oxford).

Sommerfeld, A. (1949). Partial Differential Equations in Physics (Academic Press Inc., New York).

Stanic, S., Briggs, K. B., Fleisher, P., Sawyer, W. B. and Ray, R. I. (1989). “High-frequency acoustic backscattering from a coarse shell ocean bottom", J Acoust Soc Amer 85, 125136.

Stanton, T. K. (1988). "Sound scattering by cylinders of finite length. I. Fluid cylinders", J Acoust Soc Amer 83, 55-63.

Stanton, T. K. (1989). “Simple approximate formulas for backscattering of sound by spherical and elongated objects", J Acoust Soc Amer 86, 1499-1510. 
Stanton, T. K. (1992). “Sound scattering by cylinders rough elongated elastic objects. I: Means of scattered field", J Acoust Soc Amer 92, 1641-1664.

Stanton, T. K. and Chu, D. (2000). "Review and recommendations for the modelling of acoustic scattering by fluid-like elongated zooplankton: euphausids and copepods", ICES Jour of Mar Sci 57, 793-807.

Stanton, T. K. and Chu, D. (2004). "On the acoustic diffraction by the edges of benthic shells", J Acoust Soc Amer 116, 239-244.

Stanton, T. K., Nash, R. D. M., Eastwood, R. L., and Nero, R. W. (1987). “A field examination of acoustical scattering from marine organisms at $70 \mathrm{kHz}$ ", IEEE J Ocean Eng 12, 339-348.

Stanton, T. K., Chu, D., Wiebe, P.H., and Clay, C. S. (1993). “Average echoes from randomly oriented random-length finite cylinders: zooplankton models", J Acoust Soc Amer 94, 3463-3472.

Stanton, T. K., Chu, D., and Wiebe, P. H. (1998). "Sound scattering by several zooplankton groups. II. Scattering models", J Acoust Soc Amer 103, 236-253.

Stanton, T. K, Chu, D., Wiebe, P., Eastwood, R. L., and Warren, J. D. (2000). “Acoustic scattering by benthic and planktonic shelled animals", J Acoust Soc Amer 108, 535-550.

Stanton, T. K., Chu, D., and Norton, G. V. (2007). "Acoustic diffraction by deformed edges of finite length: Theory and experiment", J Acoust Soc Amer 122, 3167-3176.

Strang, G. (1986). Introduction to Applied Mathematics. (Wellesley-Cambridge Press, Wellesley, MA).

Strang, G. (1991). Calculus (Wellesley-Cambridge Press, Wellesley, MA).

Strang, W. G. and Fix, G. (1973). An Analysis of the Finite Element Method (Prentice-Hall, Upper Saddle River, NJ).

Thompson, L. L. (2006). “A review of finite-element methods for time-harmonic acoustics", J Acoust Soc Amer 119, 1315-1330.

Uberall, H., Doolittle, R. D., and McNicholas, J. V. (1966). "Use of sound pulses for a study of circumferential waves", J Acoust Soc Amer 39, 564-578.

Urick, R. J. (1983). Principles of Underwater Sound, 3rd edition (McGraw-Hill, New York). 
Waterman, P. C. (1969). "New formulation of acoustic scattering", J Acoust Soc Amer $55,1417-1429$.

Watson, G.N. (1918). "The diffraction of electric waves by the Earth", Proc roy soc (London) a95, 82-99.

Wilcox, C. H. (1956). "An expansion theorem for electromagnetic fields", Comm Pure Appl Math 9, 115-134. 